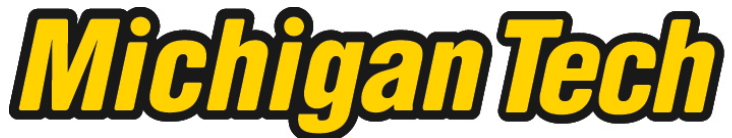 \\ Michigan Technological University Create the Future Digital Commons @ Michigan Tech
}

2015

\section{A METHOD FOR DETERMINING THE MASS COMPOSITION OF ULTRA-HIGH ENERGY COSMIC RAYS BY PREDICTING THE DEPTH OF FIRST INTERACTION OF INDIVIDUAL EXTENSIVE AIR SHOWERS}

Tolga Yapici

Michigan Technological University

Follow this and additional works at: https://digitalcommons.mtu.edu/etds

Part of the Astrophysics and Astronomy Commons, and the Physics Commons Copyright 2015 Tolga Yapici

Recommended Citation

Yapici, Tolga, "A METHOD FOR DETERMINING THE MASS COMPOSITION OF ULTRA-HIGH ENERGY COSMIC RAYS BY PREDICTING THE DEPTH OF FIRST INTERACTION OF INDIVIDUAL EXTENSIVE AIR SHOWERS", Dissertation, Michigan Technological University, 2015.

https://doi.org/10.37099/mtu.dc.etds/1019

Follow this and additional works at: https://digitalcommons.mtu.edu/etds

Part of the Astrophysics and Astronomy Commons, and the Physics Commons 


\title{
A METHOD FOR DETERMINING THE MASS COMPOSITION OF ULTRA-HIGH ENERGY COSMIC RAYS BY PREDICTING THE DEPTH OF FIRST INTERACTION OF INDIVIDUAL EXTENSIVE AIR SHOWERS
}

\author{
By \\ Tolga Yapıc1 \\ A DISSERTATION \\ Submitted in partial fulfillment of the requirements for the degree of \\ DOCTOR OF PHILOSOPHY \\ In Physics
}

MICHIGAN TECHNOLOGICAL UNIVERSITY

2015

(C) 2015 Tolga Yapıcı 
This dissertation has been approved in partial fulfillment of the requirements for the Degree of DOCTOR OF PHILOSOPHY in Physics.

\section{Department of Physics}

Dissertation Advisor: Dr. Brian E. Fick

Committee Member: Dr. David F. Nitz

Committee Member: Dr. Petra Huentemeyer

Committee Member: Dr. Nilufer Onder

Department Chair: Dr. Ravindra Pandey 


\section{Contents}

Page

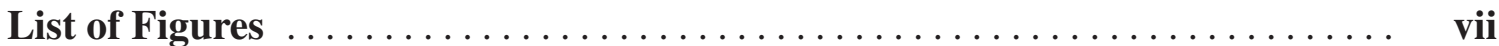

List of Tables $\ldots \ldots \ldots \ldots \ldots \ldots \ldots \ldots \ldots \ldots \ldots \ldots \ldots \ldots \ldots \ldots \ldots \ldots$

Acknowledgement ................................ xxvii

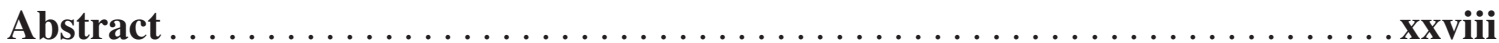

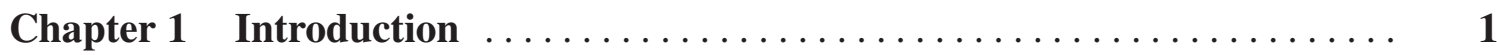

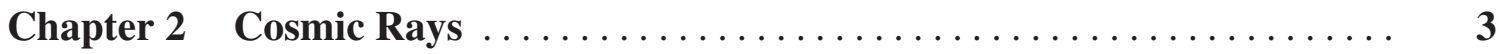

2.1 Early History of Cosmic Rays . . . . . . . . . . . . . . . . . . . . . 3

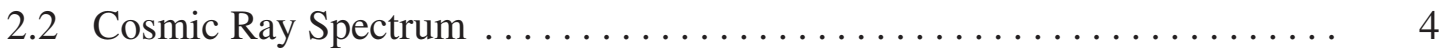

2.3 Acceleration of Cosmic Rays $\ldots \ldots \ldots \ldots \ldots \ldots \ldots \ldots \ldots \ldots \ldots \ldots \ldots \ldots \ldots \ldots$

2.3.1 Top-down Mechanism ......................... 6

2.3.2 Bottom-up Mechanism $\ldots \ldots \ldots \ldots \ldots \ldots \ldots \ldots \ldots \ldots \ldots \ldots$

2.4 Composition scenarios of Ultra High Energy Cosmic Rays . . . . . . . . . 8

Chapter 3 Extensive Air Showers . . . . . . . $\ldots \ldots \ldots \ldots \ldots \ldots \ldots \ldots \ldots$

3.1 Morphology of an Extensive Air Shower .................... 10 


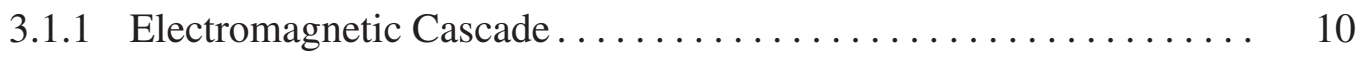

3.1.2 Hadronic Cascade. . . . . . . . . . . . . . . . . . . . . 12

3.2 Detecting Extensive Air Showers $\ldots \ldots \ldots \ldots \ldots \ldots \ldots \ldots \ldots \ldots \ldots \ldots \ldots \ldots \ldots$

3.3 Extensive Air Shower Simulations . . . . . . . . . . . . . . 15

Chapter 4 The Pierre Auger Observatory $\ldots \ldots \ldots \ldots \ldots \ldots \ldots \ldots \ldots$

4.1 The Surface Detector $\ldots \ldots \ldots \ldots \ldots \ldots \ldots \ldots \ldots \ldots \ldots \ldots \ldots \ldots \ldots$

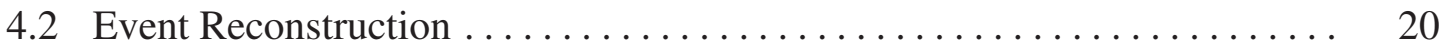

4.2.1 Muon Production Depth (MPD) Profile ............... 22

Chapter 5 Artificial Neural Networks $\ldots \ldots \ldots \ldots \ldots \ldots \ldots \ldots \ldots \ldots$

5.1 Morphology of Artificial Neural Networks $\ldots \ldots \ldots \ldots \ldots \ldots \ldots \ldots$

5.2 Artificial Neural Network Software packages ............... 26

Chapter 6 Developing a First Interaction Length Prediction Model . . . . . . . 27

6.1 Searching for proxies of first interaction lengths . . . . . . . . . . 27

6.2 Artificial Neural Network based Prediction Model ................ 30

6.2.1 Training and Testing of Artificial Neural Network based prediction model ............................. 30

6.2.2 Testing the Prediction Model based on Artificial Neural Network

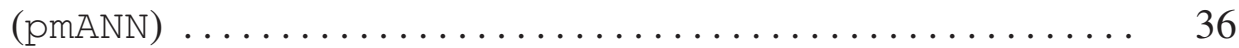


6.2.2.1 Determining Uncertainties with Bootstrap Method ..... 36

6.2.2.2 Determining model input values for mean free path ..... 37

6.2.2.3 Testing pmANN with simulated showers .......... 37

6.2.3 Determining a General Correction Factor ............. 56

Chapter 7 Mixed Cosmic Ray Scenario and Composition Analysis . . . . . . 61

7.1 Finding the break point for a two-component composition $\ldots \ldots \ldots \ldots 2$

7.2 Computing break points for a two-component simulated dataset . . . . 67

7.2.1 Application of Bootstrap Method with Monte Carlo Number Gener-

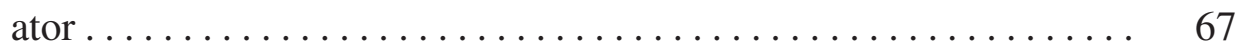

7.3 Testing the Break Points $\ldots \ldots \ldots \ldots \ldots \ldots \ldots \ldots \ldots \ldots \ldots \ldots \ldots$

7.3.1 Testing $\mathrm{p}-\mathrm{CNO}$ composition $\ldots \ldots \ldots \ldots \ldots \ldots \ldots \ldots \ldots \ldots \ldots \ldots \ldots \ldots \ldots$

7.3.2 Testing $\mathrm{p}-\mathrm{Fe}$ composition $\ldots \ldots \ldots \ldots \ldots \ldots \ldots \ldots \ldots \ldots \ldots \ldots \ldots \ldots$

7.3.3 Testing CNO-Fe composition $\ldots \ldots \ldots \ldots \ldots \ldots \ldots \ldots \ldots \ldots$

7.4 Choosing the proper break point $\ldots \ldots \ldots \ldots \ldots \ldots \ldots \ldots \ldots \ldots$

7.5 Calculating the Corrections due to BPM $\ldots \ldots \ldots \ldots \ldots \ldots \ldots \ldots$

Chapter 8 Application of pmANN+BPM for 2 component simulated showers . 96

8.1 Application of pmANN+BPM to QGSJET Showers $\ldots \ldots \ldots \ldots \ldots \ldots .97$

8.2 Application of pmANN+BPM to SIBYLL Showers $\ldots \ldots \ldots \ldots \ldots \ldots$ 
8.3 Application of pmANN+BPM to EPOS Showers . . . . . . . . . . . . 109

Chapter 9 Conclusion and Outlook $\ldots \ldots \ldots \ldots \ldots \ldots \ldots \ldots \ldots \ldots \ldots$

References.............................. 116

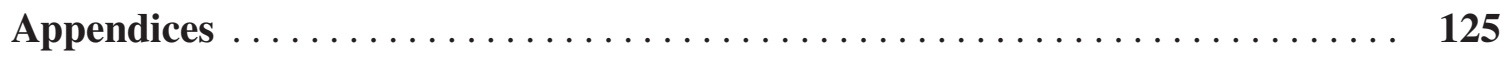

Appendix A Results of pmANN+BPM $\ldots \ldots \ldots \ldots \ldots \ldots \ldots \ldots \ldots \ldots \ldots \ldots$

A.1 Results of pmAnN+BPM for QGSJET $\ldots \ldots \ldots \ldots \ldots \ldots \ldots \ldots \ldots \ldots \ldots$

A.2 Results of pmANN+BPM for SIBYLL $\ldots \ldots \ldots \ldots \ldots \ldots \ldots \ldots \ldots \ldots \ldots$

A.3 Results of pmAnN+BPM for EPOS $\ldots \ldots \ldots \ldots \ldots \ldots \ldots \ldots \ldots \ldots \ldots$ 


\section{List of Figures}

Figure 2.1 Scaled flux of cosmic rays $\ldots \ldots \ldots \ldots \ldots \ldots \ldots \ldots \ldots \ldots$

Figure 2.2 Energy of protons as they propagate propagate through CMB $\ldots \ldots 7$

Figure $2.3 \mathrm{X}_{\max }$ and $\mathrm{RMS}\left(\mathrm{X}_{\max }\right)$ change with respect to energy $\ldots \ldots \ldots \ldots .9$

Figure 2.4 GZK horizon of proton, helium, $\mathrm{CNO}$ and iron $\ldots \ldots \ldots \ldots \ldots$

Figure 3.1 An illustration of Brehmstrahlung process, an electron deflected in the presence of a nucleus. ....................... 11

Figure 3.2 Heitler Model illustration of an electromagnetic cascade........ 11

Figure 3.3 A sample shower longitudinal profile $\ldots \ldots \ldots \ldots \ldots \ldots \ldots \ldots$

Figure 3.4 Cross-section measurements by different experiments and calculations by different hadronic interaction models $\ldots \ldots \ldots \ldots \ldots \ldots \ldots$

Figure 4.1 Layout of the Pierre Auger Observatory $\ldots \ldots \ldots \ldots \ldots \ldots \ldots$

Figure 4.2 A water Cherenkov tank deployed in the Pierre Auger Observatory. 18

Figure 4.3 A sample signal trace from an observed event............... 19

Figure 4.4 Calibration of Surface Detector with Fluorescence Detectors . . . . . 20

Figure 4.5 Integrated signals in an observed event. ............. 21

Figure 4.6 LDF fit to integrated signals in an observed event $\ldots \ldots \ldots \ldots \ldots 21$

Figure 4.7 Geometric transformation for muon production depth profile . . . . . 22

Figure 4.8 A sample reconstructed muon production depth profile ........ 23 
Figure 5.1 Illustration of an ANN. Three characteristics input are chosen with a single layer of 5 neurons to characterize a single output. . . . . . . . 25

Figure 6.1 Plots for reconstructed $\mathrm{R}, \mathrm{X}_{\max }$ and $\mathrm{X}_{1}$ values for iron and proton

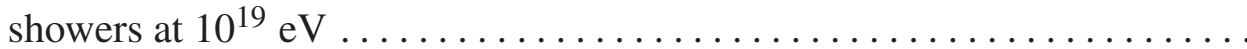

Figure 6.2 Profile plot of $\delta \mathrm{X}$ with respect to $\mathrm{X} 37$ and $1^{\text {st }}$ order polynomial fit for showers generated by different hadronic interaction models (a) for proton, (b) for $\mathrm{CNO}$, (c) for iron. . . . . . . . . . . . . . . . . .

Figure 6.3 A sample shower profile with $\theta=40$ to illustrate the characteristic

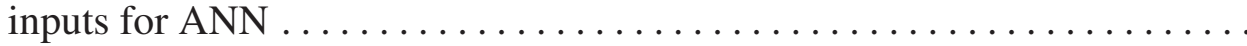

Figure 6.4 Diagram of weights for different hadronic interaction models, QGSJET in red, EPOS in blue and SIBYLL in green. Results of the training after combining the showers from three hadronic interaction models is shown in

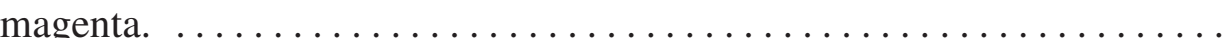

Figure 6.5 Diagram showing the influence of adding $\mathrm{Xd} \mu \mathrm{mx}$ to the characteristic inputs for showers of combination of three hadronic interaction model. Bottom two blocks (green and blue) show the weights for training in the absence of the electromagnetic components' information............

Figure 6.6 Relative difference between the simulation values and ANN prediction for different energy bins of QGSJET proton showers. . . . . . . . . .

Figure 6.7 Distribution of mean-free paths constructed by bootstrap method for proton showers generated by QGSJET hadronic interaction model at four different energy bins . . . . . . . . . . . . . . . . . .

Figure 6.8 Relative difference between the simulation values and ANN prediction for different energy bins of $\mathrm{CNO}$ showers. . . . . . . . . . . .

Figure 6.9 Distribution of mean-free paths constructed by bootstrap method for CNO showers generated by QGSJET hadronic interaction model at four

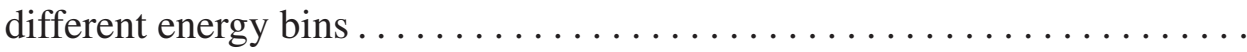


Figure 6.10 Relative difference between the simulation values and ANN prediction for different energy bins of iron showers. . . . . . . . . . . . . .

Figure 6.11 Distribution of mean-free paths constructed by bootstrap method for iron showers generated by QGSJET hadronic interaction model at four different energy bins . . . . . . . . . . . . . . . . . . .

Figure 6.12 Relative difference between the simulation values and ANN prediction for different energy bins of proton showers................

Figure 6.13 Distribution of mean-free paths constructed by bootstrap method for proton showers generated by SIBYLL hadronic interaction model at four

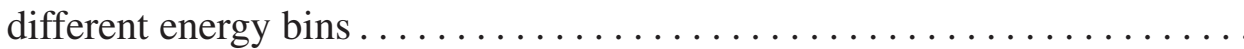

Figure 6.14 Relative difference between the simulation values and ANN prediction for different energy bins of proton showers..............

Figure 6.15 Distribution of mean-free paths constructed by bootstrap method for CNO showers generated by SIBYLL hadronic interaction model at four

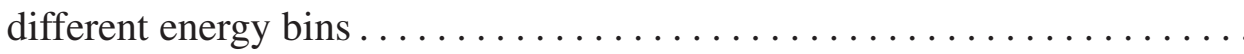

Figure 6.16 Relative difference between the simulation values and ANN prediction for different energy bins of proton showers...............

Figure 6.17 Distribution of mean-free paths constructed by bootstrap method for iron showers generated by SIBYLL hadronic interaction model at four

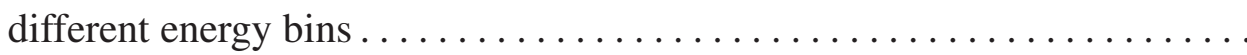

Figure 6.18 Relative difference between the simulation values and ANN prediction for different energy bins of proton showers..............

Figure 6.19 Distribution of mean-free paths constructed by bootstrap method for proton showers generated by EPOS hadronic interaction model at four different energy bins . . . . . . . . . . . . . . . . . . . . . . 
Figure 6.20 Relative difference between the simulation values and ANN prediction for different energy bins of proton showers.............. 52

Figure 6.21 Distribution of mean-free paths constructed by bootstrap method for $\mathrm{CNO}$ showers generated by EPOS hadronic interaction model at four

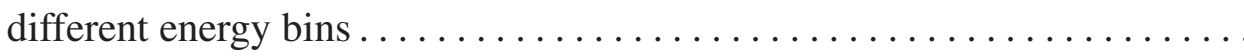

Figure 6.22 Relative difference between the simulation values and ANN prediction for different energy bins of proton showers................

Figure 6.23 Distribution of mean-free paths constructed by bootstrap method for iron showers generated by EPOS hadronic interaction model at four

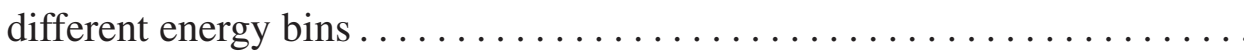

Figure 7.1 A sample distribution of $\mathrm{X}_{1}$ values for a mixed composition in semi-

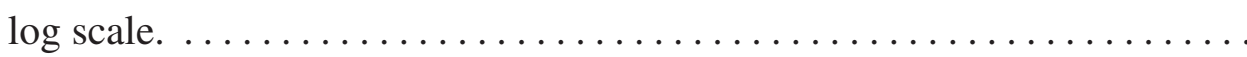

Figure 7.2 Solution of Eqn.Eqn 7.6 for $\alpha$ for p-CNO (black), p-Fe (red), CNO$\mathrm{Fe}$ (blue) mixtures for different mixture ratios $(\beta=0.90) \ldots \ldots \ldots \ldots$

Figure 7.3 Fraction of particles after a selected break point for different light particle fraction. Proton is used for light particles and $\mathrm{CNO}$ are used for heavy particles. Break point on each individual line is the solution of Eqn. Eqn 7.6 for a certain light particle fraction. $(\beta=0.90) \ldots \ldots \ldots \ldots \ldots \ldots$

Figure 7.4 Fraction of particles after a selected break point for different light particle fraction. Proton is used for light particles and Fe is used for heavy particles. Break point on each individual line is the solution of Eqn. Eqn 7.6 for a certain light particle fraction. $(\beta=0.90) \ldots \ldots \ldots \ldots \ldots \ldots$

Figure 7.5 Fraction of particles after a selected break point for different light particle fraction. CNO are used for light particles and Fe is used for heavy particles. Break point on each individual line is the solution of Eqn. Eqn 7.6 for a certain light particle fraction. $(\beta=0.90) \ldots \ldots \ldots \ldots \ldots \ldots$ 
Figure 7.6 Fraction of particles (both light and heavy particles) after a selected break point for different light particle fraction for (a) p-CNO mixture, (b) p-Fe mixture, (c) CNO-Fe mixture. Break point on each individual line is the solution of Eqn. Eqn 7.6 for a certain light particle fraction. $(\beta=0.90)$.

Figure 7.7 Results of bootstrap analysis for different $\beta$ and $\gamma$ values applied to QGSJET simulations. Results for proton first interaction length are shown in (a) for $90 \%$ proton - $10 \%$ CNO mixture and (b) for $60 \%$ proton - $40 \%$

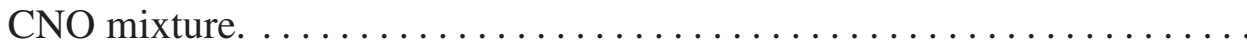

Figure 7.8 Results of bootstrap analysis for different $\beta$ and $\gamma$ values applied to SIBYLL simulations. Results for proton first interaction length are shown in (a) for $90 \%$ proton - $10 \%$ CNO mixture and (b) for $60 \%$ proton $-40 \%$

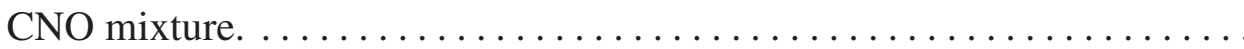

Figure 7.9 Results of bootstrap analysis for different $\beta$ and $\gamma$ values applied to EPOS simulations. Results for proton first interaction length are shown in (a) for $90 \%$ proton $-10 \% \mathrm{CNO}$ mixture and (b) for $60 \%$ proton $-40 \% \mathrm{CNO}$

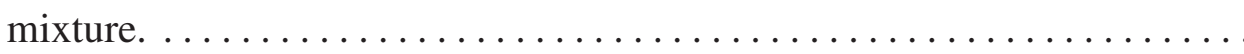

Figure 7.10 Results of different break points for different proton and CNO mixtures using QGSJET interaction model values. . . . . . . . . . . . .

Figure 7.11 Results of different break points for different proton and CNO mixtures using SIBYLL interaction model values. . . . . . . . . . . . 72

Figure 7.12 Results of different break points for different proton and CNO mixtures using EPOS interaction model values. . . . . . . . . . . . . .

Figure 7.13 Results of bootstrap analysis for different $\beta$ and $\gamma$ values applied to QGSJET simulations. Results for proton first interaction length are shown in (a) for $90 \%$ proton - $10 \%$ iron mixture and (b) for $60 \%$ proton - $40 \%$ iron mixture. 
Figure 7.14 Results of bootstrap analysis for different $\beta$ and $\gamma$ values applied to SIBYLL simulations. Results for proton first interaction length are shown in (a) for $90 \%$ proton - $10 \%$ iron mixture and (b) for $60 \%$ proton $-40 \%$ iron mixture.

Figure 7.15 Results of bootstrap analysis for different $\beta$ and $\gamma$ values applied to EPOS simulations. Results for proton first interaction length are shown in (a) for $90 \%$ proton $-10 \%$ iron mixture and (b) for $60 \%$ proton $-40 \%$ iron

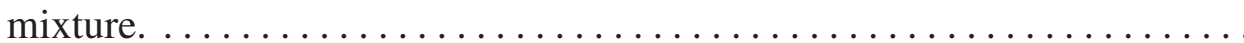

Figure 7.16 Results of different break points for different proton and iron mixtures using QGSJET interaction model values. ................

Figure 7.17 Results of different break points for different proton and iron mixtures using SIBYLL interaction model values. . . . . . . . . . . . 80

Figure 7.18 Results of different break points for different proton and iron mixtures using EPOS interaction model values. . . . . . . . . . . . . .

Figure 7.19 Results of bootstrap analysis for different $\beta$ and $\gamma$ values applied to QGSJET simulations. Results for proton first interaction length are shown in (a) for $90 \% \mathrm{CNO}-10 \%$ iron mixture and (b) for $60 \% \mathrm{CNO}-40 \%$ iron mixture.

Figure 7.20 Results of bootstrap analysis for different $\beta$ and $\gamma$ values applied to SIBYLL simulations. Results for proton first interaction length are shown in (a) for $90 \% \mathrm{CNO}-10 \%$ iron mixture and (b) for $60 \% \mathrm{CNO}-40 \%$ iron

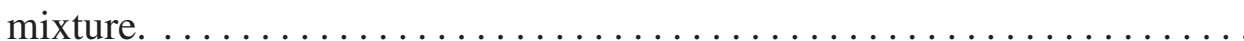

Figure 7.21 Results of bootstrap analysis for different $\beta$ and $\gamma$ values applied to EPOS simulations. Results for proton first interaction length are shown in (a) for $90 \% \mathrm{CNO}-10 \%$ iron mixture and (b) for $60 \% \mathrm{CNO}-40 \%$ iron mixture.

Figure 7.22 Results of different break points for different $\mathrm{CNO}$ and iron mixtures using QGSJET interaction model values................. 
Figure 7.23 Results of different break points for different $\mathrm{CNO}$ and iron mixtures using SIBYLL interaction model values. . . . . . . . . . . . .

Figure 7.24 Results of different break points for different $\mathrm{CNO}$ and iron mixtures

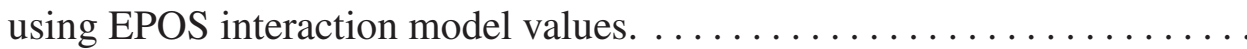

Figure 8.1 Results of pmANN+BPM for hadronic interaction model QGSJET at

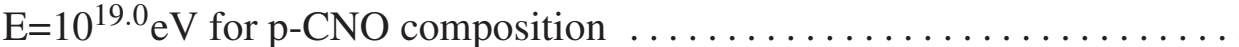

Figure 8.2 Results of pmANN+BPM for hadronic interaction model QGSJET at $\mathrm{E}=10^{19.5} \mathrm{eV}$ for $\mathrm{p}-\mathrm{CNO}$ composition $\ldots \ldots \ldots \ldots \ldots \ldots \ldots \ldots \ldots \ldots \ldots$

Figure 8.3 Results of pmANN+BPM for hadronic interaction model QGSJET at $\mathrm{E}=10^{20.0} \mathrm{eV}$ for $\mathrm{p}-\mathrm{CNO}$ composition $\ldots \ldots \ldots \ldots \ldots \ldots \ldots \ldots \ldots \ldots \ldots \ldots \ldots \ldots \ldots$

Figure 8.4 Results of pmANN+BPM for hadronic interaction model QGSJET at $\mathrm{E}=10^{19.0} \mathrm{eV}$ for $\mathrm{p}-\mathrm{Fe}$ composition . . . . . . . . . . . . . . . . 100

Figure 8.5 Results of pmANN+BPM for hadronic interaction model QGSJET at

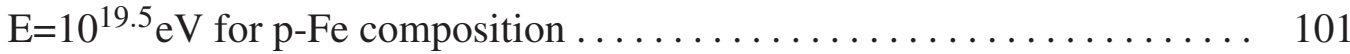

Figure 8.6 Results of pmANN+BPM for hadronic interaction model QGSJET at

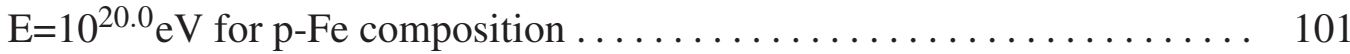

Figure 8.7 Results of pmANN+BPM for hadronic interaction model QGSJET at $\mathrm{E}=10^{19.0} \mathrm{eV}$ for $\mathrm{CNO}-\mathrm{Fe}$ composition $\ldots \ldots \ldots \ldots \ldots \ldots \ldots \ldots \ldots \ldots \ldots \ldots \ldots$

Figure 8.8 Results of pmANN+BPM for hadronic interaction model QGSJET at $\mathrm{E}=10^{19.5} \mathrm{eV}$ for $\mathrm{CNOFe}$ composition $\ldots \ldots \ldots \ldots \ldots \ldots \ldots \ldots \ldots \ldots \ldots \ldots \ldots$

Figure 8.9 Results of pmANN+BPM for hadronic interaction model QGSJET at $\mathrm{E}=10^{20.0} \mathrm{eV}$ for $\mathrm{CNOFe}$ composition $\ldots \ldots \ldots \ldots \ldots \ldots \ldots \ldots \ldots \ldots \ldots \ldots \ldots$

Figure 8.10 Results of pmANN+BPM for hadronic interaction model SIBYLL at $\mathrm{E}=10^{19.0} \mathrm{eV}$ for $\mathrm{p}-\mathrm{CNO}$ composition 
Figure 8.11 Results of pmANN+BPM for hadronic interaction model SIBYLL at $\mathrm{E}=10^{19.5} \mathrm{eV}$ for $\mathrm{p}-\mathrm{CNO}$ composition 105

Figure 8.12 Results of pmANN+BPM for hadronic interaction model SIBYLL at

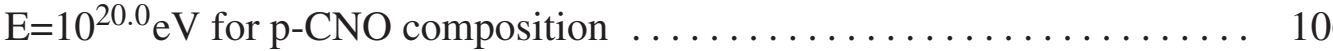

Figure 8.13 Results of pmANN+BPM for hadronic interaction model SIBYLL at

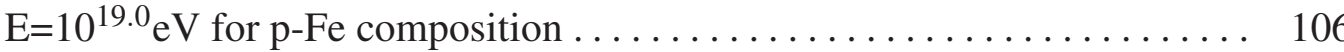

Figure 8.14 Results of pmANN+BPM for hadronic interaction model SIBYLL at

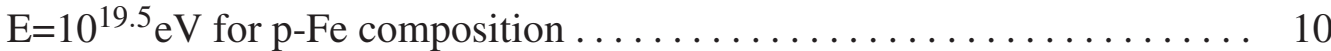

Figure 8.15 Results of pmANN+BPM for hadronic interaction model SIBYLL at $\mathrm{E}=10^{20.0} \mathrm{eV}$ for $\mathrm{p}-\mathrm{Fe}$ composition $\ldots \ldots \ldots \ldots \ldots \ldots \ldots \ldots \ldots \ldots \ldots \ldots \ldots$

Figure 8.16 Results of pmANN+BPM for hadronic interaction model SIBYLL at $\mathrm{E}=10^{19.0} \mathrm{eV}$ for $\mathrm{CNO}-\mathrm{Fe}$ composition $\ldots \ldots \ldots \ldots \ldots \ldots \ldots \ldots \ldots \ldots$

Figure 8.17 Results of pmANN+BPM for hadronic interaction model SIBYLL at $\mathrm{E}=10^{19.5} \mathrm{eV}$ for $\mathrm{CNOFe}$ composition $\ldots \ldots \ldots \ldots \ldots \ldots \ldots \ldots \ldots \ldots$

Figure 8.18 Results of pmANN+BPM for hadronic interaction model SIBYLL at $\mathrm{E}=10^{20.0} \mathrm{eV}$ for $\mathrm{CNOFe}$ composition . . . . . . . . . . . . . . 109

Figure 8.19 Results of pmANN+BPM for hadronic interaction model EPOS at $\mathrm{E}=10^{19.0} \mathrm{eV}$ for $\mathrm{p}-\mathrm{CNO}$ composition $\ldots \ldots \ldots \ldots \ldots \ldots \ldots \ldots \ldots \ldots \ldots$

Figure 8.20 Results of pmANN+BPM for hadronic interaction model EPOS at

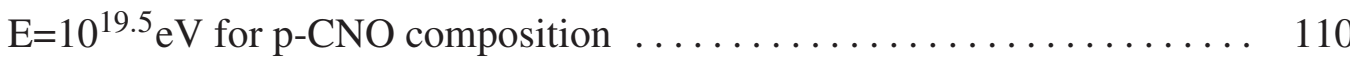

Figure 8.21 Results of pmANN+BPM for hadronic interaction model EPOS at $\mathrm{E}=10^{20.0} \mathrm{eV}$ for $\mathrm{p}-\mathrm{CNO}$ composition

Figure 8.22 Results of pmANN+BPM for hadronic interaction model EPOS at $\mathrm{E}=10^{19.0} \mathrm{eV}$ for $\mathrm{p}-\mathrm{Fe}$ composition 
Figure 8.23 Results of pmANN+BPM for hadronic interaction model EPOS at

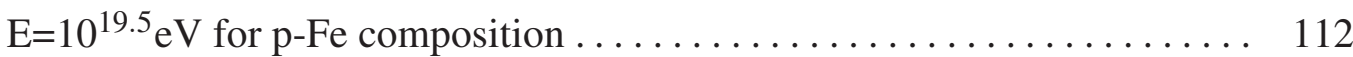

Figure 8.24 Results of pmANN+BPM for hadronic interaction model EPOS at $\mathrm{E}=10^{20.0} \mathrm{eV}$ for $\mathrm{p}-\mathrm{Fe}$ composition $\ldots \ldots \ldots \ldots \ldots \ldots \ldots \ldots \ldots \ldots \ldots \ldots \ldots \ldots \ldots \ldots$

Figure 8.25 Results of pmANN+BPM for hadronic interaction model EPOS at $\mathrm{E}=10^{19.0} \mathrm{eV}$ for $\mathrm{CNO}-\mathrm{Fe}$ composition $\ldots \ldots \ldots \ldots \ldots \ldots \ldots \ldots \ldots \ldots \ldots$

Figure 8.26 Results of pmANN+BPM for hadronic interaction model EPOS at $\mathrm{E}=10^{19.5} \mathrm{eV}$ for $\mathrm{CNOFe}$ composition $\ldots \ldots \ldots \ldots \ldots \ldots \ldots \ldots \ldots \ldots \ldots \ldots$

Figure 8.27 Results of pmANN+BPM for hadronic interaction model EPOS at $\mathrm{E}=10^{20.0} \mathrm{eV}$ for $\mathrm{CNOFe}$ composition $\ldots \ldots \ldots \ldots \ldots \ldots \ldots \ldots \ldots \ldots \ldots \ldots \ldots$ 


\section{List of Tables}

Table 3.1 Cosmic Ray Experiments $\ldots \ldots \ldots \ldots \ldots \ldots \ldots \ldots \ldots \ldots \ldots \ldots \ldots$

Table 6.1 List of characteristic inputs tested $\ldots \ldots \ldots \ldots \ldots \ldots \ldots \ldots \ldots$

Table 6.2 List of selected characteristic inputs $\ldots \ldots \ldots \ldots \ldots \ldots \ldots \ldots$

Table 6.3 Mean-free paths of cosmic ray primaries for four energy bins. Values were obtained through the distributions of the first interaction lenghts of the generated showers using three different hadronic interaction models. ....

Table 6.4 Expected value of mean-free path of different primaries for shower simulated with three hadronic interaction models at four energy bins. Simulation values, pmANN values and biases were in $\mathrm{g} / \mathrm{cm}^{2} \ldots \ldots \ldots \ldots \ldots$

Table 6.5 Weighted average of biases of different primaries for shower simulated with three hadronic interaction models at four energy bins . . . . . .

Table 6.6 Corrected expected values of mean-free paths of different primaries for shower simulated with three hadronic interaction models at four energy bins. Simulation values and pmANN values were in $\mathrm{g} / \mathrm{cm}^{2} \ldots \ldots \ldots \ldots$.

Table 7.1 Results of different breaking points for various fractions of proton CNO mixture. Mean free path values of proton and $\mathrm{p}$ were computed with Monte Carlo Number Generator fed by QGSJET values. Differences were calculated using the simulation values

Table 7.2 Results of different breaking points for various fractions of proton CNO mixture. Mean free path values of proton and CNO were computed with Monte Carlo Number Generator fed by SIBYLL values. Differences were calculated using the simulation values $\ldots \ldots \ldots \ldots \ldots \ldots \ldots \ldots$ 
Table 7.3 Results of different breaking points for various fractions of proton CNO mixture. Mean free path values of proton and CNO were computed with Monte Carlo Number Generator fed by EPOS values. Differences were calculated using the simulation values $\ldots \ldots \ldots \ldots \ldots \ldots \ldots$

Table 7.4 Results of different breaking points for various fractions of proton iron mixture. Mean free path values of proton and iron were computed with Monte Carlo Number Generator fed by QGSJET values. Differences were calculated using the simulation values $\ldots \ldots \ldots \ldots \ldots \ldots \ldots$

Table 7.5 Results of different breaking points for various fractions of proton iron mixture. Mean free path values of proton and iron were computed with Monte Carlo Number Generator fed by SIBYLL values. Differences were calculated using the simulation values $\ldots \ldots \ldots \ldots \ldots \ldots \ldots$

Table 7.6 Results of different breaking points for various fractions of proton iron mixture. Mean free path values of proton and iron were computed with Monte Carlo Number Generator fed by EPOS values. Differences were calculated using the simulation values $\ldots \ldots \ldots \ldots \ldots \ldots \ldots \ldots$

Table 7.7 Results of different breaking points for various fractions of CNO iron mixture. Mean free path values of $\mathrm{CNO}$ and iron were computed with Monte Carlo Number Generator fed by QGSJET values. Differences were calculated using the simulation values $\ldots \ldots \ldots \ldots \ldots \ldots \ldots \ldots \ldots \ldots \ldots$

Table 7.8 Results of different breaking points for various fractions of CNO iron mixture. Mean free path values of $\mathrm{CNO}$ and iron were computed with Monte Carlo Number Generator fed by SIBYLL values. Differences were calculated using the simulation values $\ldots \ldots \ldots \ldots \ldots \ldots \ldots \ldots \ldots \ldots \ldots$

Table 7.9 Results of different breaking points for various fractions of CNO iron mixture. Mean free path values of $\mathrm{CNO}$ and iron were computed with Monte Carlo Number Generator fed by EPOS values. Differences were calculated using the simulation values $\ldots \ldots \ldots \ldots \ldots \ldots \ldots \ldots \ldots \ldots$ 
Table 7.10 Weighted averages of biases of the light particle (printed in bold) for three different mixtures of primary particles $\left(\mathrm{m}_{p}<\mathrm{m}_{C N O}<\mathrm{m}_{F e}\right) \ldots \ldots$

Table 7.11 Weighted averages of biases of the heavy particle (printed in bold) for three different mixtures of primary particles $\left(\mathrm{m}_{p}<\mathrm{m}_{C N O}<\mathrm{m}_{F e}\right) \ldots \ldots$

Table 8.1 Correction values of different primaries for shower simulated with three hadronic interaction models at four energy bins $\ldots \ldots \ldots \ldots \ldots \ldots$.

Table A.1 Calculated simulation and ANN values using pmANN+BPM for $\mathrm{p}$ in p-CNO mixture of QGSJET showers at $\log (\mathrm{E} / \mathrm{eV})=18.75$.

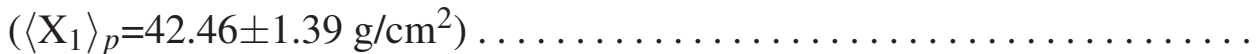

Table A.2 Calculated simulation and ANN values using pmANN+BPM for CNO in p-CNO mixture of QGSJET showers at $\log (\mathrm{E} / \mathrm{eV})=18.75$

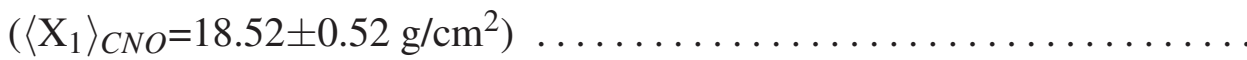

Table A.3 Calculated simulation and ANN values using pmANN+BPM for $\mathrm{p}$ in p-CNO mixture of QGSJET showers at $\log (\mathrm{E} / \mathrm{eV})=19.00$

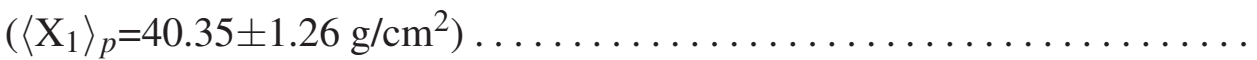

Table A.4 Calculated simulation and ANN values using pmANN+BPM for CNO in $\mathrm{p}$-CNO mixture of QGSJET showers at $\log (\mathrm{E} / \mathrm{eV})=19.00$

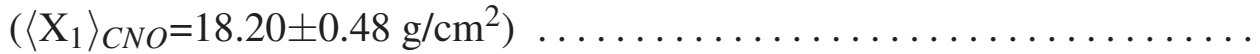

Table A.5 Calculated simulation and ANN values using pmANN+BPM for $\mathrm{p}$ in p-CNO mixture of QGSJET showers at $\log (\mathrm{E} / \mathrm{eV})=19.50$

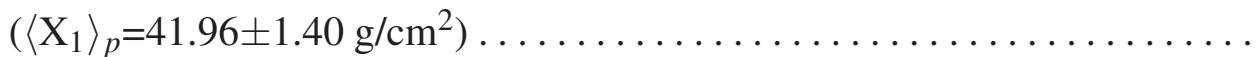

Table A.6 Calculated simulation and ANN values using pmANN+BPM for CNO in p-CNO mixture of QGSJET showers at $\log (\mathrm{E} / \mathrm{eV})=19.50$

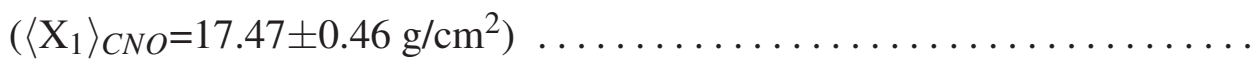

Table A.7 Calculated simulation and ANN values using pmANN+BPM for $\mathrm{p}$ in p-CNO mixture of QGSJET showers at $\log (\mathrm{E} / \mathrm{eV})=20.00$

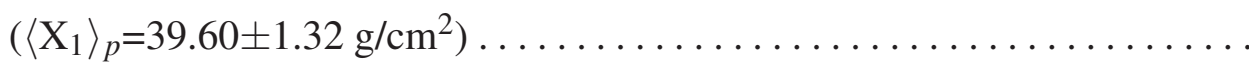


Table A.8 Calculated simulation and ANN values using pmANN+BPM for CNO in $\mathrm{p}$-CNO mixture of QGSJET showers at $\log (\mathrm{E} / \mathrm{eV})=20.00$

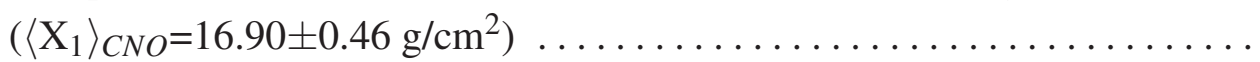

Table A.9 Calculated simulation and ANN values using pmANN+BPM for $\mathrm{p}$ in p-iron mixture of QGSJET showers at $\log (\mathrm{E} / \mathrm{eV})=18.75$

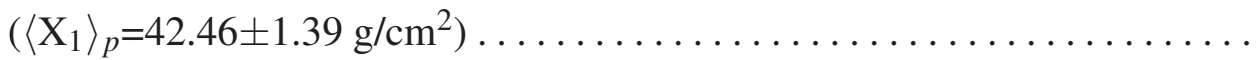

Table A.10Calculated simulation and ANN values using pmANN+BPM for iron in p-iron mixture of QGSJET showers at $\log (\mathrm{E} / \mathrm{eV})=18.75$

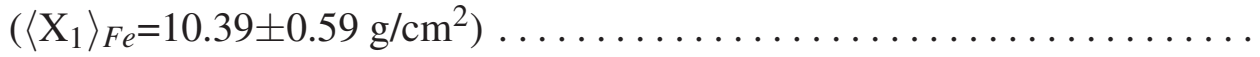

Table A.11 Calculated simulation and ANN values using pmANN+BPM for $\mathrm{p}$ in p-iron mixture of QGSJET showers at $\log (\mathrm{E} / \mathrm{eV})=19.00$

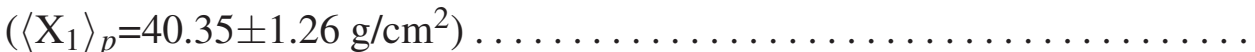

Table A.12Calculated simulation and ANN values using pmANN+BPM for iron in p-iron mixture of QGSJET showers at $\log (\mathrm{E} / \mathrm{eV})=19.00$

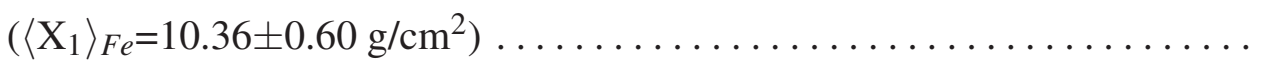

Table A.13 Calculated simulation and ANN values using pmANN+BPM for $\mathrm{p}$ in p-iron mixture of QGSJET showers at $\log (\mathrm{E} / \mathrm{eV})=19.50$

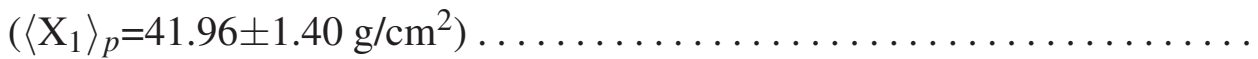

Table A.14Calculated simulation and ANN values using pmANN+BPM for iron in p-iron mixture of QGSJET showers at $\log (\mathrm{E} / \mathrm{eV})=19.50$

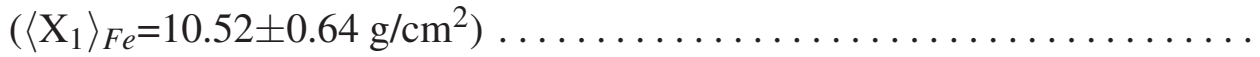

Table A.15 Calculated simulation and ANN values using pmANN+BPM for $\mathrm{p}$ in p-iron mixture of QGSJET showers at $\log (\mathrm{E} / \mathrm{eV})=20.00$

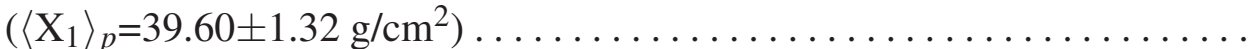

Table A.16Calculated simulation and ANN values using pmANN+BPM for iron in p-iron mixture of QGSJET showers at $\log (\mathrm{E} / \mathrm{eV})=20.00$

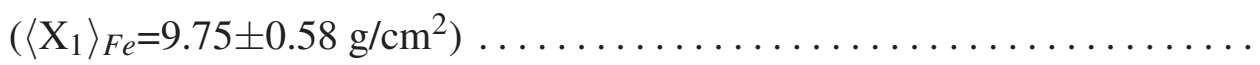


Table A.17 Calculated simulation and ANN values using pmANN+BPM for CNO in CNO-iron mixture of QGSJET showers at $\log (\mathrm{E} / \mathrm{eV})=18.75$

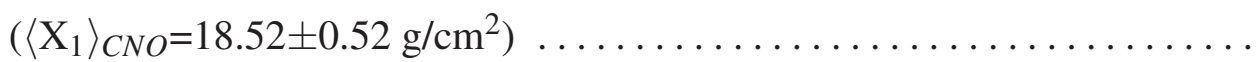

Table A.18Calculated simulation and ANN values using pmANN+BPM for iron in CNO-iron mixture of QGSJET showers at $\log (\mathrm{E} / \mathrm{eV})=18.75$

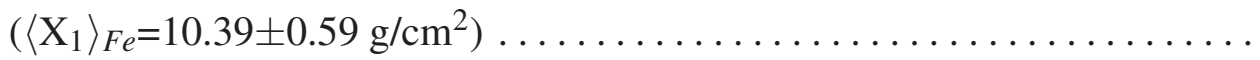

Table A.19Calculated simulation and ANN values using pmANN+BPM for CNO in CNO-iron mixture of QGSJET showers at $\log (\mathrm{E} / \mathrm{eV})=19.00$

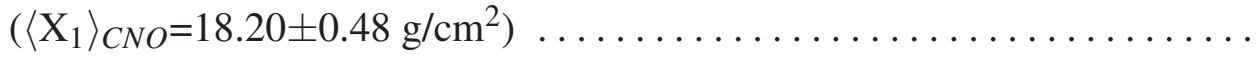

Table A.20Calculated simulation and ANN values using pmANN+BPM for iron in CNO-iron mixture of QGSJET showers at $\log (\mathrm{E} / \mathrm{eV})=19.00$

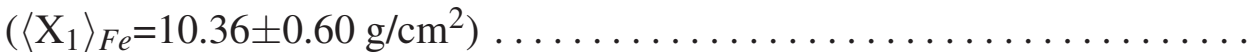

Table A.21 Calculated simulation and ANN values using pmANN+BPM for CNO in CNO-iron mixture of QGSJET showers at $\log (\mathrm{E} / \mathrm{eV})=19.50$

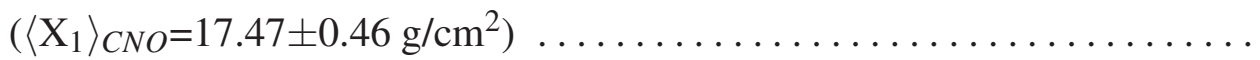

Table A.22 Calculated simulation and ANN values using pmANN+BPM for iron in CNO-iron mixture of QGSJET showers at $\log (\mathrm{E} / \mathrm{eV})=19.50$ $\left(\left\langle\mathrm{X}_{1}\right\rangle_{F e}=10.52 \pm 0.64 \mathrm{~g} / \mathrm{cm}^{2}\right)$

Table A.23 Calculated simulation and ANN values using pmANN+BPM for CNO in CNO-iron mixture of QGSJET showers at $\log (\mathrm{E} / \mathrm{eV})=20.00$

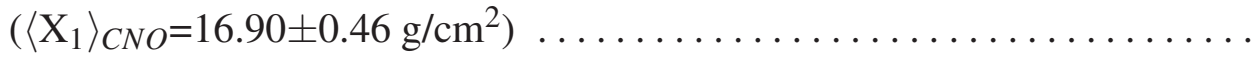

Table A.24Calculated simulation and ANN values using pmANN+BPM for iron in CNO-iron mixture of QGSJET showers at $\log (\mathrm{E} / \mathrm{eV})=20.00$

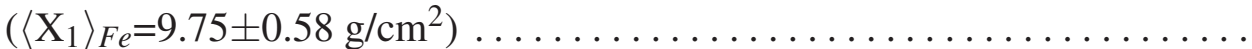

Table A.25 Calculated simulation and ANN values using pmANN+BPM for $\mathrm{p}$ in $\mathrm{p}$-CNO mixture of SIBYLL showers at $\log (\mathrm{E} / \mathrm{eV})=18.75$

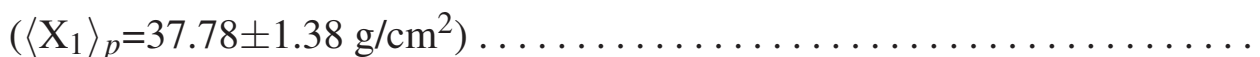


Table A.26Calculated simulation and ANN values using pmANN+BPM for CNO in $\mathrm{p}-\mathrm{CNO}$ mixture of SIBYLL showers at $\log (\mathrm{E} / \mathrm{eV})=18.75$

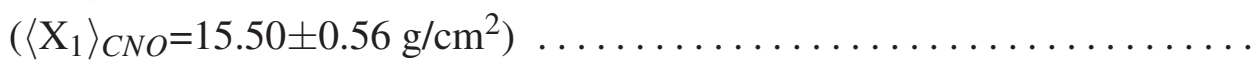

Table A.27 Calculated simulation and ANN values using pmANN+BPM for $\mathrm{p}$ in $\mathrm{p}-\mathrm{CNO}$ mixture of SIBYLL showers at $\log (\mathrm{E} / \mathrm{eV})=19.00$

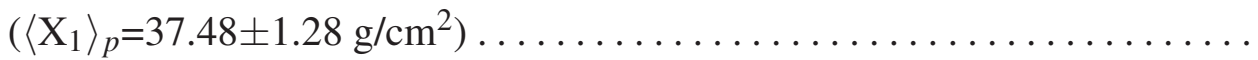

Table A.28Calculated simulation and ANN values using pmANN+BPM for CNO in $\mathrm{p}$-CNO mixture of SIBYLL showers at $\log (\mathrm{E} / \mathrm{eV})=19.00$

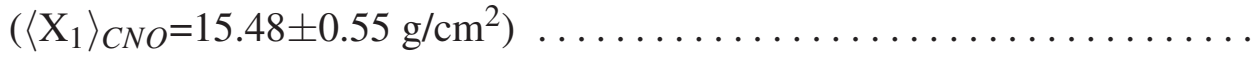

Table A.29Calculated simulation and ANN values using pmANN+BPM for $\mathrm{p}$ in p-CNO mixture of SIBYLL showers at $\log (\mathrm{E} / \mathrm{eV})=19.50$

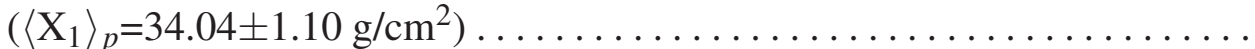

Table A.30 Calculated simulation and ANN values using pmANN+BPM for CNO in $\mathrm{p}-\mathrm{CNO}$ mixture of SIBYLL showers at $\log (\mathrm{E} / \mathrm{eV})=19.50$

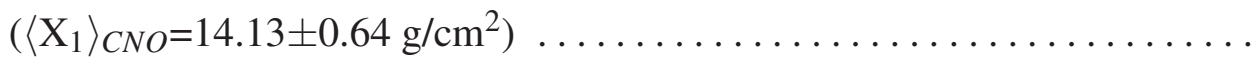

Table A.31 Calculated simulation and ANN values using pmANN+BPM for $\mathrm{p}$ in p-CNO mixture of SIBYLL showers at $\log (\mathrm{E} / \mathrm{eV})=20.00$

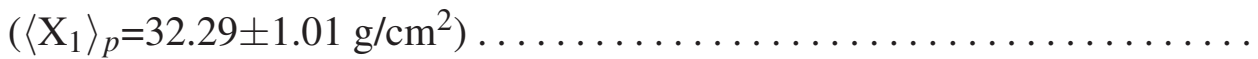

Table A.32 Calculated simulation and ANN values using pmANN+BPM for CNO in $\mathrm{p}$-CNO mixture of SIBYLL showers at $\log (\mathrm{E} / \mathrm{eV})=20.00$

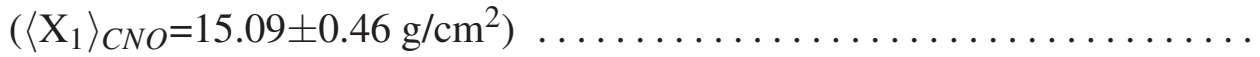

Table A.33 Calculated simulation and ANN values using pmANN+BPM for $p$ in p-iron mixture of SIBYLL showers at $\log (\mathrm{E} / \mathrm{eV})=18.75$

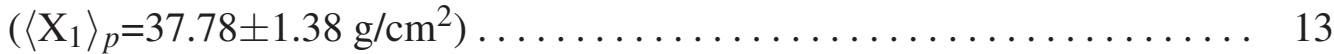

Table A.34Calculated simulation and ANN values using pmANN+BPM for iron in p-iron mixture of SIBYLL showers at $\log (\mathrm{E} / \mathrm{eV})=18.75$

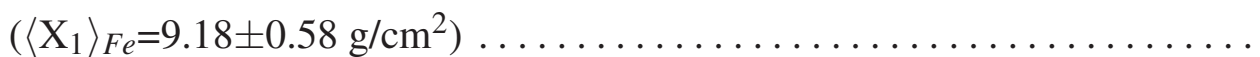


Table A.35 Calculated simulation and ANN values using pmANN+BPM for $\mathrm{p}$ in p-iron mixture of SIBYLL showers at $\log (\mathrm{E} / \mathrm{eV})=19.00$

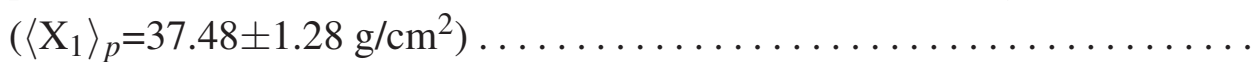

Table A.36Calculated simulation and ANN values using pmANN+BPM for iron in p-iron mixture of SIBYLL showers at $\log (\mathrm{E} / \mathrm{eV})=19.00$ $\left(\left\langle\mathrm{X}_{1}\right\rangle_{F e}=9.42 \pm 0.56 \mathrm{~g} / \mathrm{cm}^{2}\right)$

Table A.37 Calculated simulation and ANN values using pmANN+BPM for $p$ in p-iron mixture of SIBYLL showers at $\log (\mathrm{E} / \mathrm{eV})=19.50$ $\left(\left\langle\mathrm{X}_{1}\right\rangle_{p}=34.04 \pm 1.10 \mathrm{~g} / \mathrm{cm}^{2}\right) \ldots \ldots \ldots \ldots \ldots \ldots \ldots \ldots \ldots \ldots \ldots \ldots \ldots \ldots \ldots \ldots \ldots \ldots \ldots$

Table A.38Calculated simulation and ANN values using pmANN+BPM for iron in p-iron mixture of SIBYLL showers at $\log (\mathrm{E} / \mathrm{eV})=19.50$

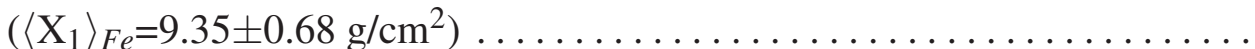

Table A.39Calculated simulation and ANN values using pmANN+BPM for $\mathrm{p}$ in p-iron mixture of SIBYLL showers at $\log (\mathrm{E} / \mathrm{eV})=20.00$

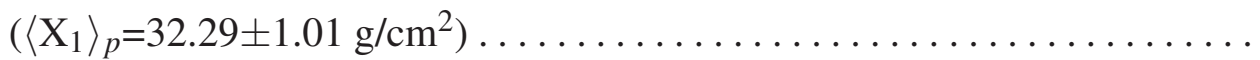

Table A.40Calculated simulation and ANN values using pmANN+BPM for iron in p-iron mixture of SIBYLL showers at $\log (\mathrm{E} / \mathrm{eV})=20.00$ $\left(\left\langle\mathrm{X}_{1}\right\rangle_{F e}=9.16 \pm 1.03 \mathrm{~g} / \mathrm{cm}^{2}\right)$

Table A.41 Calculated simulation and ANN values using pmANN+BPM for CNO in CNO-iron mixture of SIBYLL showers at $\log (\mathrm{E} / \mathrm{eV})=18.75$

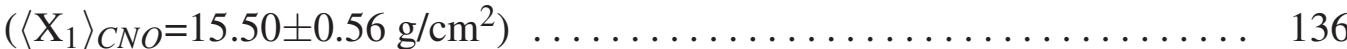

Table A.42 Calculated simulation and ANN values using pmANN+BPM for iron in CNO-iron mixture of SIBYLL showers at $\log (\mathrm{E} / \mathrm{eV})=18.75$

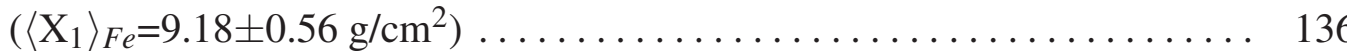

Table A.43 Calculated simulation and ANN values using pmANN+BPM for CNO in CNO-iron mixture of SIBYLL showers at $\log (\mathrm{E} / \mathrm{eV})=19.00$

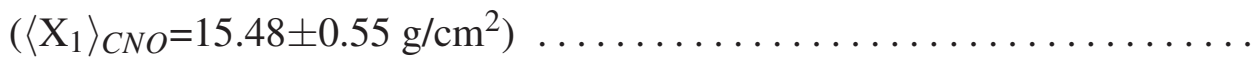


Table A.44Calculated simulation and ANN values using pmANN+BPM for iron in CNO-iron mixture of SIBYLL showers at $\log (\mathrm{E} / \mathrm{eV})=19.00$

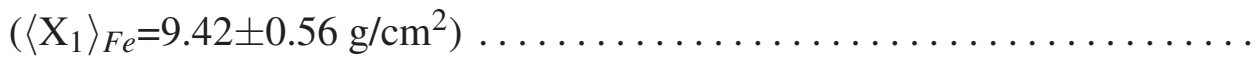

Table A.45 Calculated simulation and ANN values using pmANN+BPM for CNO in CNO-iron mixture of SIBYLL showers at $\log (\mathrm{E} / \mathrm{eV})=19.50$

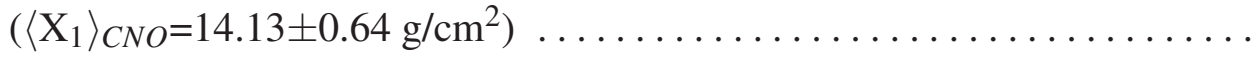

Table A.46Calculated simulation and ANN values using pmANN+BPM for iron in CNO-iron mixture of SIBYLL showers at $\log (\mathrm{E} / \mathrm{eV})=19.50$

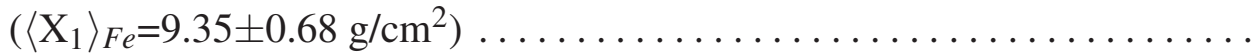

Table A.47 Calculated simulation and ANN values using pmANN+BPM for CNO in CNO-iron mixture of SIBYLL showers at $\log (\mathrm{E} / \mathrm{eV})=20.00$

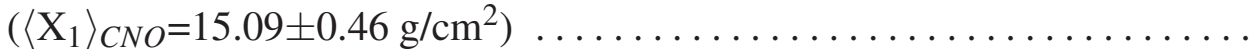

Table A.48Calculated simulation and ANN values using pmANN+BPM for iron in CNO-iron mixture of SIBYLL showers at $\log (\mathrm{E} / \mathrm{eV})=20.00$

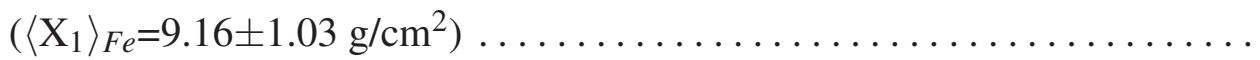

Table A.49Calculated simulation and ANN values using pmANN+BPM for CNO in $\mathrm{p}-\mathrm{CNO}$ mixture of EPOS showers at $\log (\mathrm{E} / \mathrm{eV})=18.75$ $\left(\left\langle\mathrm{X}_{1}\right\rangle_{C N O}=16.10 \pm 0.55 \mathrm{~g} / \mathrm{cm}^{2}\right)$

Table A.50 Calculated simulation and ANN values using pmANN+BPM for CNO in $\mathrm{p}$-CNO mixture of EPOS showers at $\log (\mathrm{E} / \mathrm{eV})=18.75$

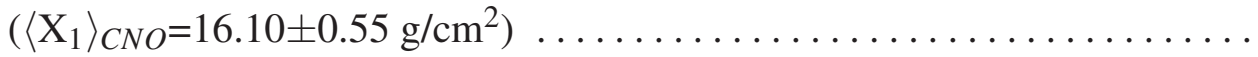

Table A.51 Calculated simulation and ANN values using pmANN+BPM for CNO in $\mathrm{p}$-CNO mixture of EPOS showers at $\log (\mathrm{E} / \mathrm{eV})=19.00$

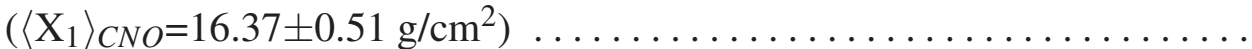

Table A.52 Calculated simulation and ANN values using pmANN+BPM for CNO in $\mathrm{p}$-CNO mixture of EPOS showers at $\log (\mathrm{E} / \mathrm{eV})=19.00$

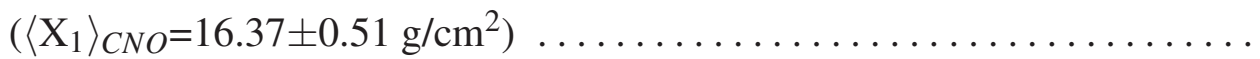


Table A.53 Calculated simulation and ANN values using pmANN+BPM for $\mathrm{p}$ in $\mathrm{p}-\mathrm{CNO}$ mixture of EPOS showers at $\log (\mathrm{E} / \mathrm{eV})=19.50$

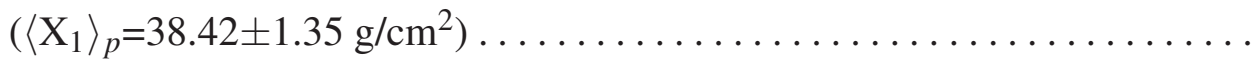

Table A.54Calculated simulation and ANN values using pmANN+BPM for CNO in $\mathrm{p}$-CNO mixture of EPOS showers at $\log (\mathrm{E} / \mathrm{eV})=19.50$

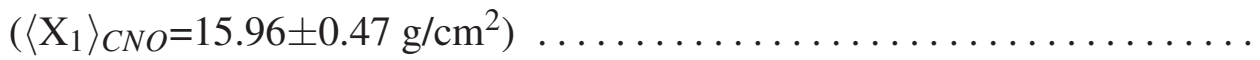

Table A.55 Calculated simulation and ANN values using pmANN+BPM for $p$ in p-CNO mixture of EPOS showers at $\log (\mathrm{E} / \mathrm{eV})=20.00$ $\left(\left\langle\mathrm{X}_{1}\right\rangle_{p}=37.72 \pm 1.38 \mathrm{~g} / \mathrm{cm}^{2}\right) \ldots \ldots \ldots \ldots \ldots \ldots \ldots \ldots \ldots \ldots \ldots \ldots \ldots \ldots \ldots \ldots \ldots \ldots \ldots$

Table A.56Calculated simulation and ANN values using pmANN+BPM for CNO in $\mathrm{p}$-CNO mixture of EPOS showers at $\log (\mathrm{E} / \mathrm{eV})=20.00$

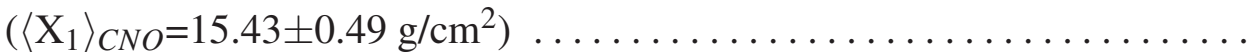

Table A.57 Calculated simulation and ANN values using pmANN+BPM for $\mathrm{p}$ in p-iron mixture of EPOS showers at $\log (\mathrm{E} / \mathrm{eV})=18.75$

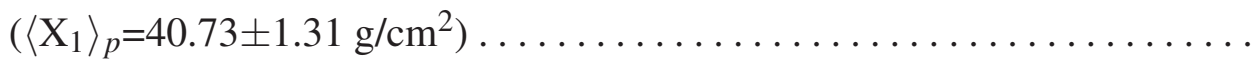

Table A.58Calculated simulation and ANN values using pmANN+BPM for iron in $\mathrm{p}-\mathrm{CNO}$ mixture of EPOS showers at $\log (\mathrm{E} / \mathrm{eV})=18.75$ $\left(\left\langle\mathrm{X}_{1}\right\rangle_{F e}=11.09 \pm 0.75 \mathrm{~g} / \mathrm{cm}^{2}\right)$

Table A.59 Calculated simulation and ANN values using pmANN+BPM for $p$ in p-iron mixture of EPOS showers at $\log (\mathrm{E} / \mathrm{eV})=19.00$

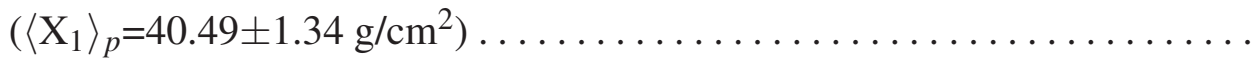

Table A.60Calculated simulation and ANN values using pmANN+BPM for iron in $\mathrm{p}$-CNO mixture of EPOS showers at $\log (\mathrm{E} / \mathrm{eV})=19.00$

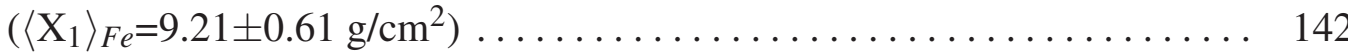

Table A.61 Calculated simulation and ANN values using pmANN+BPM for $\mathrm{p}$ in p-iron mixture of EPOS showers at $\log (\mathrm{E} / \mathrm{eV})=19.50$

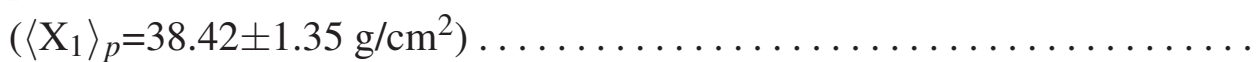


Table A.62 Calculated simulation and ANN values using pmANN+BPM for iron in $\mathrm{p}$-CNO mixture of EPOS showers at $\log (\mathrm{E} / \mathrm{eV})=19.50$

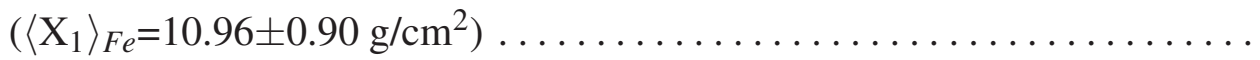

Table A.63 Calculated simulation and ANN values using pmANN+BPM for $\mathrm{p}$ in p-iron mixture of EPOS showers at $\log (\mathrm{E} / \mathrm{eV})=20.00$ $\left(\left\langle\mathrm{X}_{1}\right\rangle_{p}=37.72 \pm 1.38 \mathrm{~g} / \mathrm{cm}^{2}\right)$

Table A.64Calculated simulation and ANN values using pmANN+BPM for iron in $\mathrm{p}$-CNO mixture of EPOS showers at $\log (\mathrm{E} / \mathrm{eV})=20.00$

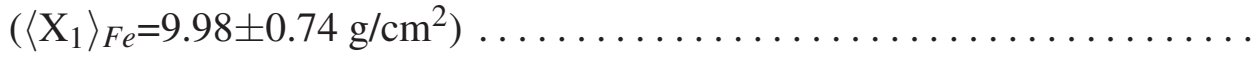

Table A.65 Calculated simulation and ANN values using pmANN+BPM for CNO in CNO-iron mixture of EPOS showers at $\log (\mathrm{E} / \mathrm{eV})=18.75$

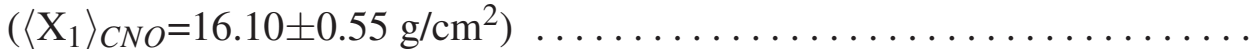

Table A.66Calculated simulation and ANN values using pmANN+BPM for iron in CNO-iron mixture of EPOS showers at $\log (\mathrm{E} / \mathrm{eV})=18.75$

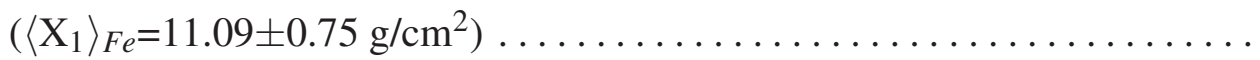

Table A.67 Calculated simulation and ANN values using pmANN+BPM for CNO in CNO-iron mixture of EPOS showers at $\log (\mathrm{E} / \mathrm{eV})=19.00$ $\left(\left\langle\mathrm{X}_{1}\right\rangle_{C N O}=16.37 \pm 0.51 \mathrm{~g} / \mathrm{cm}^{2}\right)$

Table A.68 Calculated simulation and ANN values using pmANN+BPM for iron in CNO-iron mixture of EPOS showers at $\log (\mathrm{E} / \mathrm{eV})=19.00$

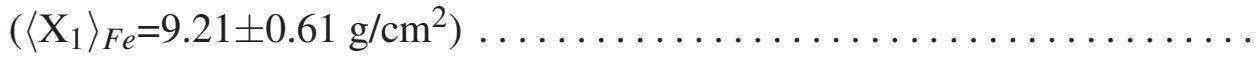

Table A.69 Calculated simulation and ANN values using pmANN+BPM for CNO in CNO-iron mixture of EPOS showers at $\log (\mathrm{E} / \mathrm{eV})=19.50$

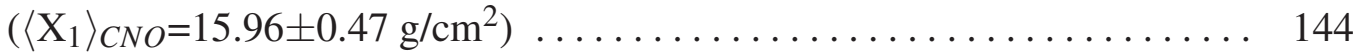

Table A.70Calculated simulation and ANN values using pmANN+BPM for iron in CNO-iron mixture of EPOS showers at $\log (\mathrm{E} / \mathrm{eV})=19.50$

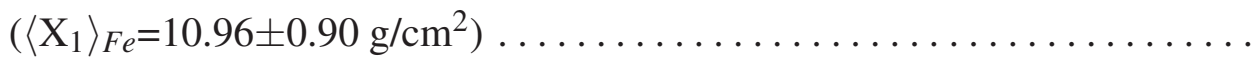


Table A.71 Calculated simulation and ANN values using pmANN+BPM for CNO in CNO-iron mixture of EPOS showers at $\log (\mathrm{E} / \mathrm{eV})=20.00$

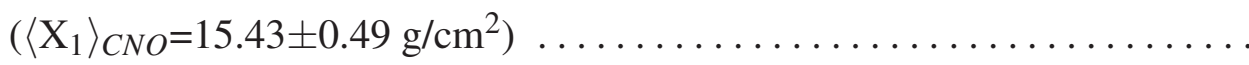

Table A.72Calculated simulation and ANN values using pmANN+BPM for iron in CNO-CNO mixture of EPOS showers at $\log (\mathrm{E} / \mathrm{eV})=20.00$

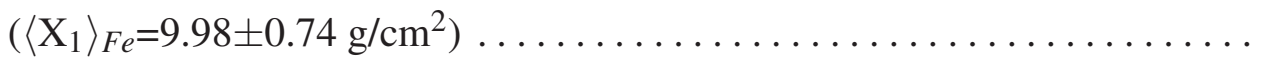




\section{Acknowledgement}

I would like express my gratitude for the support and encouragement provided by my academic advisor, Dr. Brian Fick. Without his help, this work would not mature.

I also would like to help Dr. David Nitz for the support and training in electronics. While working with him on electronics for Pierre Auger Observatory Research and Development Array (RDA), I learned about software development for electronics from scretch.

I thank Department of Physics at Michigan Technological University and Department of Energy for their financial support during the completion of this work

Last but not the least, I thank my wife, Nazmiye Yapıcı for believing in me, supporting in me at any moment and help me get through the obstacles. 


\begin{abstract}
Particle accelerators have been used to characterize the properties of particle and subatomic particles. The most advanced particle accelerators built, LHC, can run at $10^{17} \mathrm{eV}$. It is not possible with current technology to accelerate particle to the energies that can be detected by cosmic ray observatories.

In the past, by the direct measurements of cosmic rays, scientists discovered sub-atomic particles. Being accelerated to energies higher than $10^{18} \mathrm{eV}$, cosmic rays carry important information for particle physics. We have developed a method, which is a combination of Artificial Neural Networks and simple algebraic method, that uses parameters from the extensive air shower profile to investigate the mean-free path of the cosmic rays. Method has been tested for cases including one and two component composition with success.

Due to lack of experimental measurements, the developed method was not applied to observed events. It will be possible to use the method in the future for an enhanced observatory which will measure the parameters needed.
\end{abstract}




\section{Chapter 1 Introduction}

Physics is the branch of science that tries to understand matter, energy and their interaction. At the largest scales, astrophysics examines the universe. The study of astrophysical objects (such as black holes, galaxies, stars) makes it possible to understand the formation and evolution of the universe.

Another field of physics, particle physics investigates fundamental particles and their characteristics at the smallest scales. Among these characteristics are cross-section and lifetime. The method to determine these characteristics is particle accelerators, in which accelerated particle beams collide in a well controlled environment in a detector. The maximum energy at the accelerators is limited by the available technology. The largest particle accelerator built is the Large Hadron Collider (LHC). LHC is designed for maximum of 14 $\mathrm{TeV}$ center of mass collisions ( $\sim 0.1 \mathrm{EeV}$ in rest frame for particle energies). At higher energies than LHC maximum energy, the interpretation of the properties require extrapolations. Each method of extrapolation has its own uncertainties and each method generates different trends.

The intersection of these two fields of physics is the particle astrophysics. With the astrophysical sources, particles can be accelerated to energies higher than man-made accelerators can achieve with current technology. Thus, particle astrophysics provides valuable information regarding the characteristics of fundamental particles at ultra high energies, $\mathrm{E}>10 \mathrm{EeV}$.

In this thesis, we scrutinize a possible method to predict one of the characteristic properties, mean-free path, of selected fundamental particles (that are most probable at the energy regime of interest, $10 \mathrm{EeV}<\mathrm{E}$ ) by using of ultra high energy cosmic rays. The method is based on Artificial Neural Networks and it incorporates the extensive air shower development due to ultra high energy cosmic rays. The outputs of the Artificial Neural 
Networks, first interaction lengths of cosmic ray events, are accumulated to yield the mean free path of the cosmic rays. The possibility of cosmic rays being mixed at ultra high energies was also exploited. A second method was developed for extracting information from a two component mixture. To our knowledge, this is a first attempt for predicting the mean-free path of cosmic rays at ultra high energies in a mixed composition scenario.

In Chapter 2, briefly, cosmic rays are discussed. Several composition scenarios by different approaches are summarized. Proton-CNO-iron mixture with a transition from light particle to heavy particle was selected. In Chapter 3, the manifestation of cosmic rays in the atmosphere, extensive air showers is explained. The components of extensive air shower is explained as well as several experimental techniques to detect the extensive air showers. In addition, the effect of extrapolation and interpretation of accelerator data at the ultra high energies is explained. It is followed by the introduction of the Pierre Auger Observatory and its detectors are explained briefly in Chapter 4. Also, the part of the shower development used in the Artificial Neural Network and its reconstruction with experiment data is briefly explained.

In Chapter 5, Artificial Neural Networks are introduced. A detailed explanation on the development of the prediction model is given in Chapter 6. The results of selected cosmic rays (proton, $\mathrm{CNO}$ and iron) are presented, and the uncertainties and biases are tabulated. The biases are then used to calculate correction factors. In Chapter 7, mixed cosmic ray scenarios are investigated using a method called break point method. Exact ratio in the mixture is not yet know, thus for the analysis possible cases are investigated with increments of 5\% in mixture. Lighter to heavier transition is assumed; however, a transition from proton to iron (with no intermediate components) is also inspected. Similar to single component analysis, correction factors due to break point method. In Chapter 8, two developed methods are combined, named pmANN+BPM. The correction values associated with individual methods are combined. Results of a series blind tests is also shown. 


\section{Chapter 2 Cosmic Rays}

\subsection{Early History of Cosmic Rays}

In the 19th century, the discovery of radiation opened a new area of physics. Charged particles created by the radioactivity were detected by a simple instrument called an electroscope. In the following decades, scientists tried to identify the sources of the radiation. In 1899, scientists detected radiation inside a thick metal container. Their conclusion was that the ionized radiation was deeply penetrating [1]. However, they could not answer whether the source is terrestrial or extra-terrestrial. In the early 1900s, scientists picked up the ionized radiation signatures even in the absence of a source. This mind boggling phenomena pushed intrigued physicist to carry out more experiments. The series of experiments conducted at different location in the absence of a radioactive material made the physicist think the Earth was the source [2].

It was not until Victor Hess performed balloon experiments in 1911 and 1913, that the source of the radiation was resolved. His experiments revealed an increase of radiation at higher altitudes $[3,4]$. He concluded that the source of the radiation was above the atmosphere. Many scientists disapproved the hypothesis of the radiation being non-terrestrial and claimed the source to be materials such as radium in the crust of the Earth. However, his findings were verified by Kolhorster in the same years [5, 6]. Establishing the fact that the sources are not Earth based, physicists started investigating the "high altitude radiation" (as Hess refered). Experiments Hess conducted revealed that Sun cannot be the only source since they detected radiation even at night times and solar eclipse [2].

Being the source of radiation in space, they were called cosmic radiation. Moreover, due to a general misunderstanding about the nature of the radiation, they were considered as photons, $\gamma$, and named "rays" [2]. Thus, these radiations were named "cosmic rays". Jacob 
Clay demonstrated the particle nature of cosmic rays with an ionization chamber on a journey over seas, since they did exhibit geomagnetic latitude dependence [7, 8]. His results showed that cosmic rays were not only $\gamma$ but also charged particles. In the following years, more experiments regarding the cosmic rays were conducted. It was the first contribution of cosmic rays physics to elementary particle physics when Anderson realized a pattern in his cloud chamber pictures [9]. The pattern suggested a positively charged particle coming from below and the detected particle had ionization density of an electron [8]. This contribution was not the last contribution of cosmic ray physics to elementary particle physics. Cosmic rays kept providing important knowledge about elementary particles until the particle accelerators were built.

The development of new instruments, such as Geiger-Muller counters, helped the scientists to probe cosmic rays in more detail. In 1938, Pierre Auger, using two detectors far apart from each other, found a coincidental triggers with his detectors. The results showed the existence of secondary particles, called an extensive air shower, due to the radiation [10]. Other physicists of the era also conducted experiments, but what made Auger's work significant was the prediction of the energy of the cosmic ray $\left(\sim 10^{15} \mathrm{eV}\right)$ [11]. Later, in 1963 , Linsley reported a $10^{20} \mathrm{eV}$ event that had a striking conclusion about cosmic rays. His observation and interpretation of the event pointed that the ultra high high energy cosmic ray was a proton from an extra-galactic source [12].

The energies of the cosmic rays cover a decade of energies above $10^{10} \mathrm{eV}$. Cosmic rays up to $10^{15} \mathrm{eV}$ can be detected directly by balloon experiments [13]. However, beyond that energy (even at the limit), direct measurement is not plausible. Thus, indirect measurements incorporating the secondary particles are required. That is why the indirect measurement by Auger is a significant leap in cosmic ray physics. Despite determining the energy of the cosmic rays, the origin of the highest energy cosmic rays has yet to be answered as well as the acceleration mechanism to relativistic speeds [14].

\subsection{Cosmic Ray Spectrum}

Beyond the direct measurement regime, ground detectors were constructed to scan cosmic rays at different energies. First of its kind was the cosmic ray array built by M.I.T. group. This detector was the detector which observed the highest energy cosmic ray events until recent years [12]. Later, bigger experiments were built to probe higher energies. Some of 


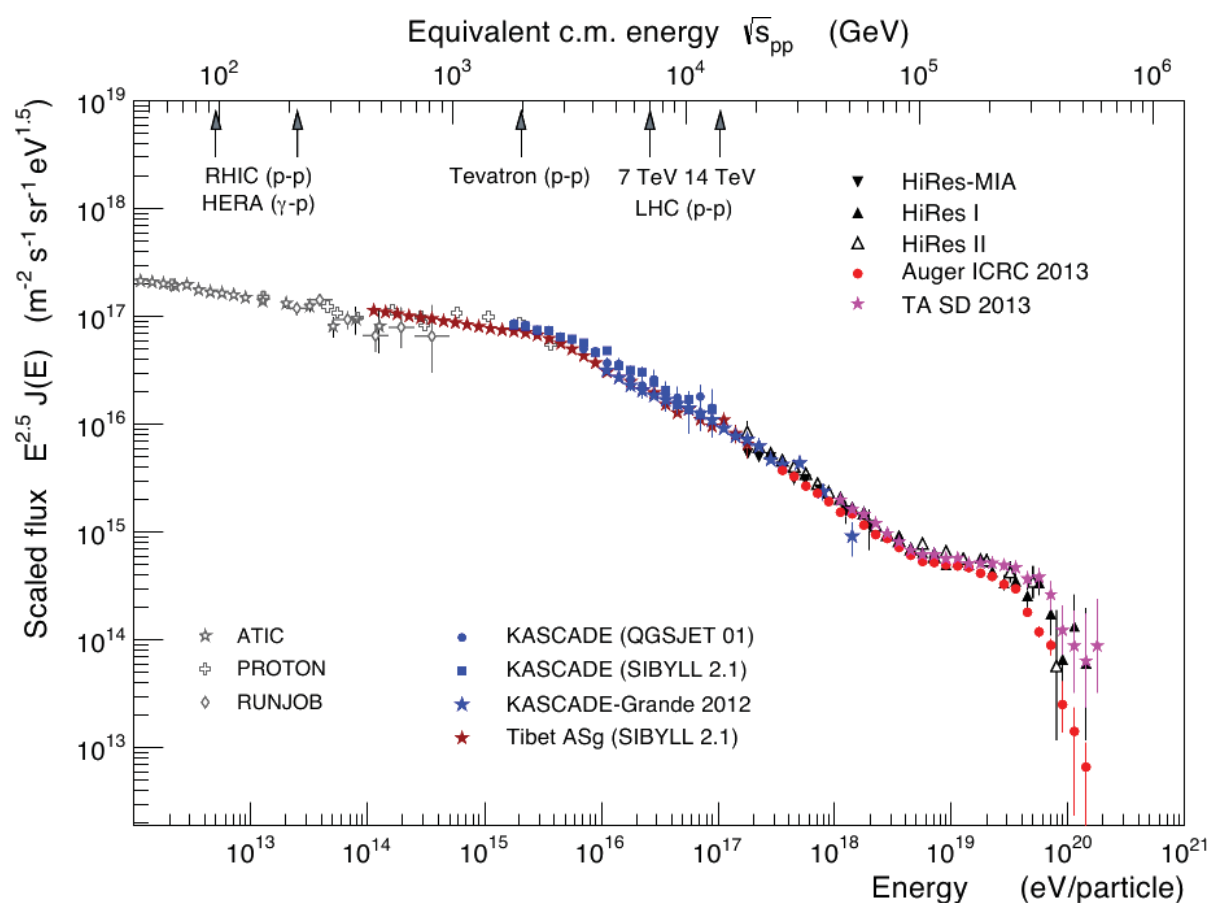

Figure 2.1: Scaled flux of cosmic rays [18]

these experiments were Yakutsk Observatory [15] and AGASA (Akeno Giant Air Shower Array) [16]. It was not only ground arrays, which used water cherenkov tanks or scintillators, but also fluorescence light detectors, looking at the energy emission by showers in the atmosphere, were built. The earliest fluorescence detector was Fly's Eye [17]. These experiments and the forthcoming ones observed cosmic ray event up to $10^{20} \mathrm{eV}$.

The cumulative result of observations is shown in Figure 2.1. The scaled flux of cosmic rays follows roughly a power law, $\mathrm{E}^{-\gamma}$, where $\gamma$ is called the spectral index. The number of events decrease drastically at high energies.

The observations revealed that 1 event per $\mathrm{m}^{2}$ per second can be observed at $10^{11} \mathrm{eV}$. At $\sim 10^{15} \mathrm{eV}$, the slope of the spectrum steepens $(\gamma: 2.7 \rightarrow 3.0)$. Because of the increase in spectral index, this feature is called "knee". At the knee, the observation rate decreases to 1 event per $\mathrm{m}^{2}$ per year. A second knee appears at $\sim 10^{17.5} \mathrm{eV}$ where the spectral index increases to 3.3. Another feature in the spectrum is at $10^{18.5} \mathrm{eV}$, at which the spectrum flattens $(\gamma: 3.3 \rightarrow 2.6)$. This is named as "the ankle". 
The spectrum shown in Figure 2.1 provides some clues about the nature of cosmic rays. The Fly's Eye Collaboration has pointed out the change from heavier to lighter particles at the ankle. Their conclusion on the characteristics of the ankle is that the mass transition in conjunction with the lack of anisotropy at these energies indicates the sources of cosmic rays change from galactic to extra-galactic [19]. The knee exposes the transition from light cosmic rays to heavier ones [20].

The sharp drop in the spectrum at $4 \times 10^{19} \mathrm{eV}$ was explained by Greisen and Zatsepin,Kuzmin independently [21, 22]. Cosmic rays at energies higher than $4 \times 10^{19} \mathrm{eV}$ (super-GZK events) interact with the "Cosmic Microwave Background (CMB)" radiation by photo disintegration via $\Delta^{*}$ resonance (Eqn 2.1, Eqn 2.2). Thus, it is unlikely to observe super-GZK events that originate more than $100 \mathrm{Mpc}$ away (Figure 2.2) in conjunction with events beyond the ankle are extra-galactic events. The suppression of cosmic rays due to the GZK effect at these energies was reported by experiments [23, 24]. For suppression, another possibility is that the sources run out of power [25].

$$
\begin{gathered}
\gamma_{C M B}+p \rightarrow \Delta^{*} \rightarrow p+\pi^{0} \\
\gamma_{C M B}+p \rightarrow \Delta^{*} \rightarrow n+\pi^{+}
\end{gathered}
$$

\subsection{Acceleration of Cosmic Rays}

The mechanism accelerating cosmic rays to the ultra high energies is not yet known. The first possible explanation came from E. Fermi in 1949 [27]. There are other arguments that can be grouped into two categories to explain the phenomena.

\subsubsection{Top-down Mechanism}

The category called top-down mechanism is for possible mechanisms in which ultra high energy cosmic rays (UHECRs) are produced by the decay of the heavy particle remnants from the early universe $[28,29]$. In addition, topological defects of the early universe may contribute to the UHECRs. These possible mechanisms are not the focus of the dissertation, and detailed information can be found in [28, 29]. 


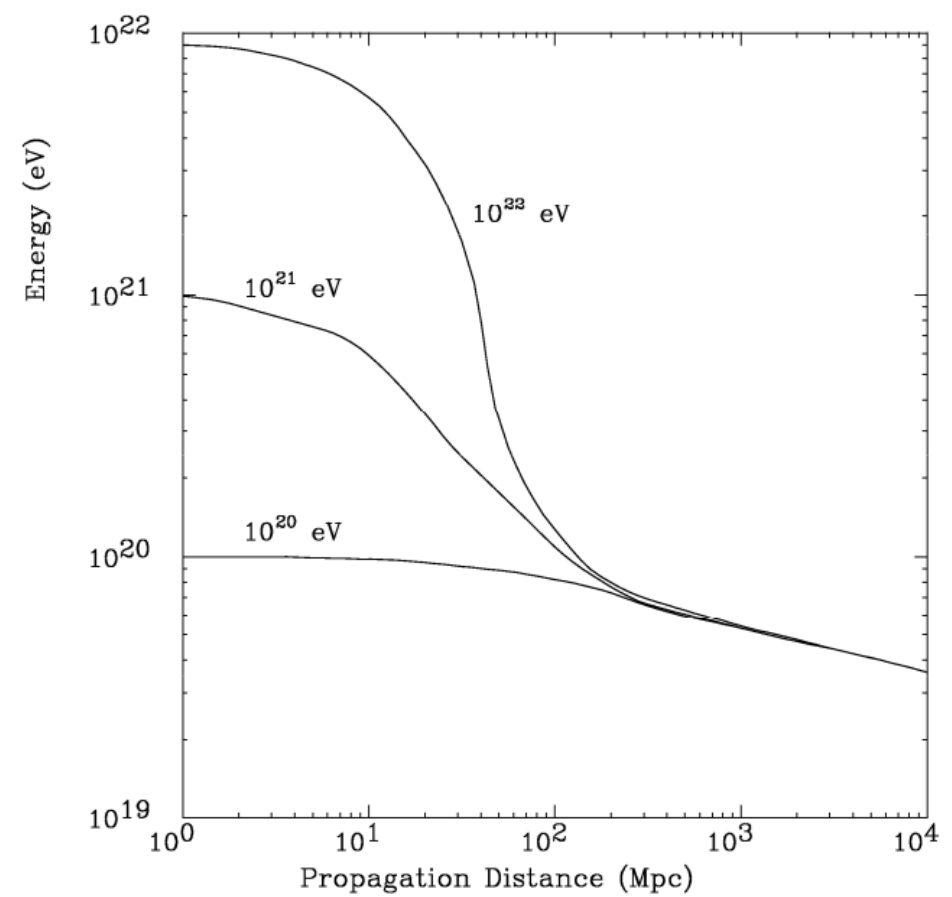

Figure 2.2: Energy of protons as they propagate propagate through CMB.Lines represent the initial proton energy at the source. [26]

As one of the signatures of top-down models, a large fraction of the cosmic rays should be neutrinos and gamma rays at very high energies. The photon and neutrino fluxes constrained the heavy particle decays [30] and the mechanisms considering the topological defects were ruled out by an earlier work [31]. Another problem with the decay of heavy particle remnants is the lifetime of these particles and their existence in the current universe [28].

\subsubsection{Bottom-up Mechanism}

Lower energy cosmic rays, protons and ions, might be accelerated by the astrophysical sites. Thus, the category of these accelerations is called "bottom-up" mechanisms. Particles gain energy by scattering off the magnetic fields in interacting plasmas at the source sites [32, 33, 34, 35]. 
A simple model for maximum energy is given by:

$$
E_{\max }=v \times Z \times L \times B
$$

where $v$ is the bulk relative velocity of the plasma, $Z$ is the charge of the particle being accelerated, $L$ is the characteristic length of the plasma and $B$ is the magnetic field in the plasma.

For cosmic rays to be accelerated to ultra high energies, large magnetic fields or large size of the source is required. Possible candidates for sources that fits the definitions are Active Galactic Nuclei (AGNs) and Gamma-ray bursts [28]. The Pierre Auger Observatory's observations of AGNs correlation with UHECR source [36] support this hypothesis.

\subsection{Composition scenarios of Ultra High Energy Cosmic Rays}

The composition of UHECRs has been always one of the compelling questions in particle astrophysics. Like the acceleration of the UHECRs, it has not been answered with certainty; however, theoretical hypotheses based on experimental results have been tested.

Experimental hypotheses rely on the interpretation of the extensive air showers generated by the cosmic rays. (See Chapter 3 for explanation of extensive air showers). Assuming heavy cosmic rays are superpositions of lighter particles with lower energy than the original particle, it is expected for heavier cosmic rays to have smaller $\mathrm{X}_{\max }$ values $\left(\mathrm{X}_{\max }\right.$ is the depth of shower maximum). A similar pattern for the variation of the $\mathrm{X}_{\max }$ is anticipated. The Pierre Auger Observatory has shown the shift from proton to heavier nuclei (Figure 2.3) [37].

The Pierre Auger Observatory then extended the study with a 4-component composition fitting to the observed data [38]. The results have suggested not a 2 component composition, proton and iron as has been proposed before. In this analysis, tested components were proton, helium, nitrogen and iron. However, the GZK horizons of these components do not prefer He to be in the composition (see Figure 2.4). 

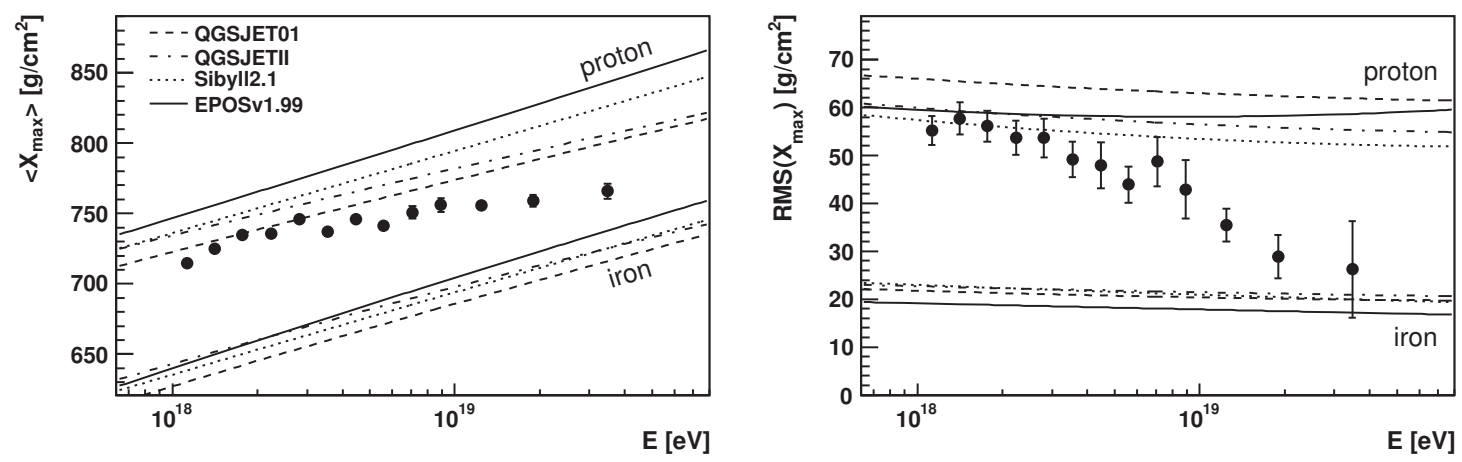

Figure 2.3: $\mathrm{X}_{\max }$ and $\mathrm{RMS}\left(\mathrm{X}_{\max }\right)$ change with respect to energy. Data points are measured by Pierre Auger Observatory [37]

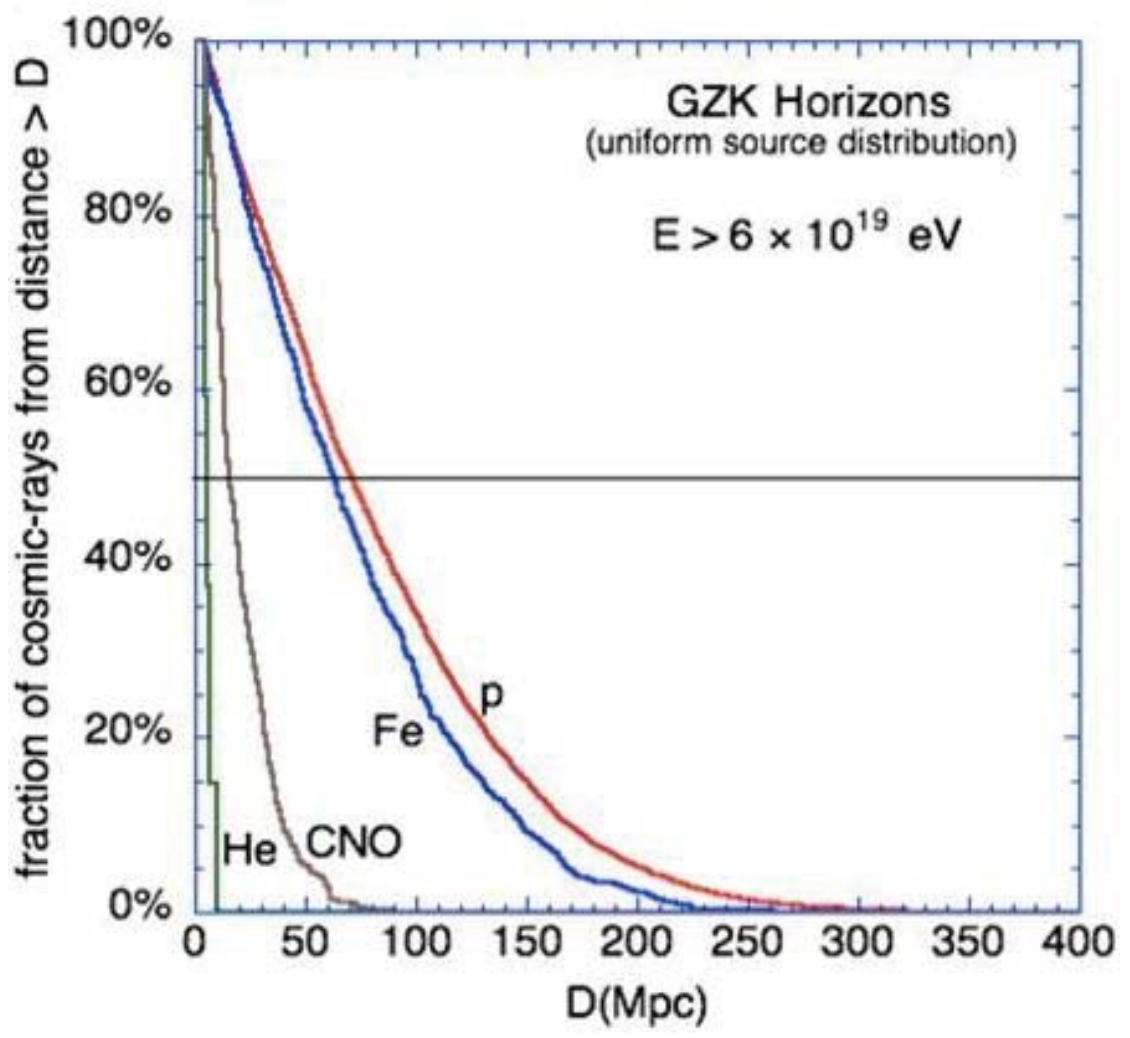

Figure 2.4: GZK horizon of proton, helium, CNO and iron [39] 


\section{Chapter 3 Extensive Air Showers}

Cosmic rays interact when they collide with atmospheric nuclei. The length of first interactions vary by energy, atmospheric density and particle type. The cosmic rays impinging on the atmosphere, also called the primary cosmic particles, may be detected by direct measurement as long as their energy is below $10^{15} \mathrm{eV}$. Primary particles with higher energies can only be detected by indirect measurements of the secondary particle created while the primary particles travels in the atmosphere.

\subsection{Morphology of an Extensive Air Shower}

Any primary particle creates an exponentially increasing number of secondary particle while traversing the atmosphere, which plays the same role as a target in an accelerator beam [40]. Depending on the type of the primary particle, an extensive air shower develops. In the case of a hadronic primary particle, a hadronic cascade is produced as well as an electromagnetic cascade. On the other hand, when an energetic photon interacts, only an electromagnetic cascade is produced.

\subsubsection{Electromagnetic Cascade}

An electromagnetic cascade is initiated by a photon that has enough energy to interact with an air nuclei in the atmosphere by pair production.

$$
\gamma+N_{\text {air }} \rightarrow e^{-}+e^{+}+N_{\text {air }}
$$




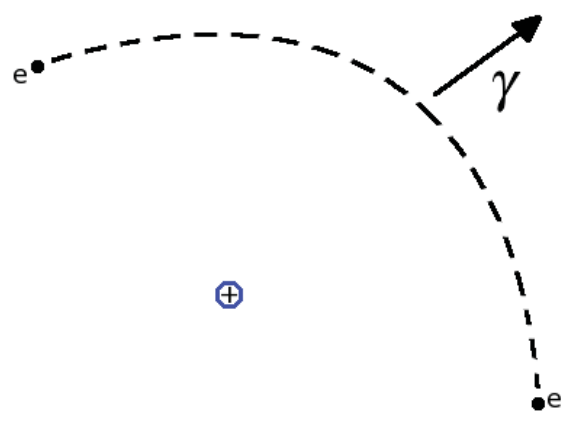

Figure 3.1: An illustration of Brehmstrahlung process, an electron deflected in the presence of a nucleus. Similar process is observed for positrons as well.

After the first interaction, the resulting $\mathrm{e}^{-}$and $\mathrm{e}^{+}$would undergo the Brehmstrahlung process and create new photons. Then, the product photon interacts again to create another $\mathrm{e}^{-}-\mathrm{e}^{+}$pair. This sequence of interactions continues until photons lose their energy down to $\mathrm{E}_{c}=85 \mathrm{MeV}$ and cannot produce any more pairs.

A simple, yet effective, model governing the electromagnetic cascade is the Heitler Model [41]. The model assumes electrons to yield two photons after traversing a constant distance, $d=\lambda \times \ln 2$, where $\lambda$ is the radiation length of photon. The distance for photons to pair-produce is assumed to be the same. An illustration of the process is shown in Figure 3.2 .

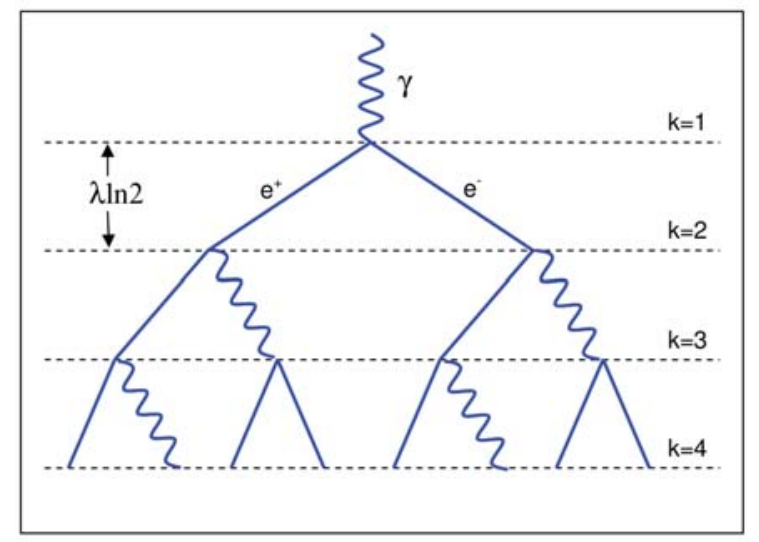

Figure 3.2: Heitler Model illustration of an electromagnetic cascade 
The energy is split equally for both processes. Under these assumptions, when the energy of the particles falls below the critical energy, the total number of particles (also the maximum number of particles) in the cascade reaches $\mathrm{N}_{\max }=\mathrm{E}_{0} / \mathrm{E}_{c}$, where $\mathrm{E}_{0}$ is the initial energy of the primary photon. Thus, the depth at which the maximum number of particles, $\mathrm{X}_{\max }$, attained is $\lambda \ln \left(\mathrm{E}_{0} / \mathrm{E}_{c}\right)$. The number of particles generated in the cascade starts decreasing after $\mathrm{X}_{\max }$ since they will be absorbed in the atmosphere.

\subsubsection{Hadronic Cascade}

The cascade of particles generated by a hadron is more complicated than an electromagnetic cascade. When a hadron, such as a proton, collides with an air molecule, a large number of mesons are created in addition to other particles like baryons. Among these mesons, pions and kaons have the highest fraction. Like the other highly unstable mesons, kaons decay into pions. These pions decay further into into particles with smaller mass on their way by the mechanisms in Eqn 3.2.

$$
\begin{aligned}
\pi^{+} & \rightarrow \mu^{+}+v_{\mu} \\
\pi^{-} & \rightarrow \mu^{-}+\bar{v}_{\mu} \\
\pi^{0} & \rightarrow 2 \gamma
\end{aligned}
$$

The processes explained above occurs along the shower development. Muons, which are generated at the initial development, with energies less than $1 \mathrm{GeV}$ decay in the atmosphere (Eqn 3.3); however, muons with energies higher than $3 \mathrm{GeV}$ can reach to the ground as a consequence of relativistic time dilation. These energy thresholds decrease for the muons

generated deeper in the atmosphere due to the decrease in distance they need to travel to earth.

$$
\begin{aligned}
& \mu^{+} \rightarrow e^{+}+v_{e}+\bar{v}_{\mu} \\
& \mu^{-} \rightarrow e^{-}+\bar{v}_{e}+v_{\mu}
\end{aligned}
$$

It should be noted that $\gamma, \mathrm{e}^{+}$and $\mathrm{e}^{-}$generated in hadronic cascades initiate their own electromagnetic cascades. A modified version of the Heitler model was developed for hadronic cascades [42]. The profile formed by the cascade of particles is called longitudinal profile (see Figure 3.3). Separately, one can consider a longitudinal profile for each component of the showers, such as electromagnetic longitudinal profile and muon production depth 
profile. The shower profiles can be best approximated by the Gaisser-Hillas function [43]:

$$
N(X)=N_{\max }\left(\frac{X-X_{0}}{X_{\max }-X_{0}}\right)^{\frac{X_{\max }-X_{0}}{\lambda}} \exp \left(\frac{X_{\max }-X}{\lambda}\right)
$$

Muons lose their energy mostly due to ionization at a rate of about $\sim 2 \mathrm{MeV}$ per $\mathrm{g} / \mathrm{cm}^{2}$ [44] whereas the electromagnetic components lose energy via inelastic scattering and pairproduction at a faster rate. The electromagnetic components provide valuable information about the energy of the cosmic rays via their energy loss mechanisms. However, only the electromagnetic components produced closer to observation level can reach the ground. On the other hand, muons generated at high altitudes reach the ground and they provide information about the characteristics of the cosmic rays. Thus, by looking at the muon production profile, one can gain information about the first interactions as well as the species of the primary cosmic rays.

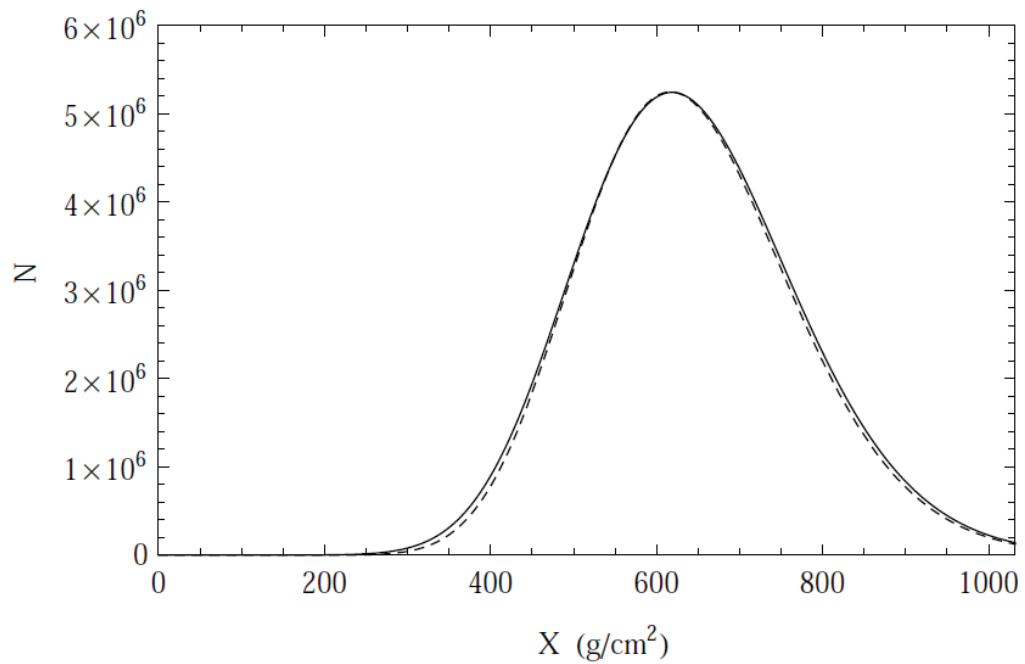

Figure 3.3: A sample shower longitudinal profile. On the y-axis, it is the number of particles; on the x-axis, it is the slant depth (a unit for the amount of matter that particle travels). 


\subsection{Detecting Extensive Air Showers}

The properties of cosmic rays can be retrieved by observing the cascades or their manifestations.

During their travel in the atmosphere, charged secondaries transfer some of their energy to the nitrogen present in the atmosphere via collisions. This process causes the nitrogen to excite and de-excite yielding fluorescence light, which results in indirect detection of the extensive air showers. The fluorescence light is in UV band $(\lambda \approx 300 \mathrm{~nm})$. With the help of light collecting detectors, the electromagnetic longitudinal profile can be obtained.

In addition to fluorescence light, the particles hitting the Earth's surface can be detected by indirect measurement techniques, such as Cherenkov Radiation in a water tank. Analogous to an aircraft moving faster than speed of sound experiencing a shock wave, the charged secondaries travelings inside a denser medium will experience as they travel faster than light, and they radiate light which can be detectable with the use of photo-multiplier tubes. Another ground based detection technique is to use scintillators. The signals of multiple detectors can be used for determining the arrival direction of the cosmic rays.

In the last decades, various experiments were built using one or both of the experimentation techniques. A summary of these observatories are listed in Table 3.1.

Table 3.1: Cosmic Ray Experiments

\begin{tabular}{lll} 
Experiment & Detector Type(s) & Years of operation \\
\hline \hline Volcano Ranch & Scintillator & $1959-1963$ \\
\hline SUGAR & Scintillator & $1968-1979$ \\
\hline Yakutsk & Scintillator & $1969-$. \\
\hline Fly's Eye & Fluorescence Detector & $1981-1992$ \\
\hline AGASA & Scintillator & $1990-2004$ \\
\hline CASA & Scintillator & $1992-2001$ \\
\hline HiRes & Fluorescence Detector & $1998-2005$ \\
\hline Pierre Auger & $\begin{array}{l}\text { Water Cherenkov } \\
\end{array}$ & + Fluorescence Detector \\
\hline Telescope Array & $\begin{array}{l}\text { Scintillator } \\
\text { + Fluorescence Detector }\end{array}$ \\
\hline
\end{tabular}




\subsection{Extensive Air Shower Simulations}

The interpretation of the extensive air showers or their manifestations is difficult. Thus, usually computer simulations are used for these purposes.

CORSIKA [45] is the most widely used software for 3D applications. Tracking and performing calculations for individual particle is computationally expensive. Thus, a feature called thinning was introduced. This process tracks particle with energies above a certain value thoroughly. Particles with energies below the threshold are represented by a single particle randomly selected among these particles. Other particles are removed and a weight is assigned to the remaining particle to account for the energy conservation.

On the occasion that only the longitudinal profile is of importance, a simpler hybrid approach can be used. CONEX is a 1D version of CORSIKA. Above the threshold energy, particles are treated the same way as in CORSIKA. Below the threshold, a numerical solution based on hadronic cascade equation are employed. What makes CONEX advantageous is the use of these analytical equations that shortens the computation time of the shower simulation. More detailed information on CONEX can be read from [46].

In both software, the first couple of interactions, which occur at high energies, require important interaction parameters, such as the cross-section and inelasticity. These interaction parameters are calculated using the knowledge attained from particle accelerators. However, for UHECRs, the energy at which the first interactions happen is orders of magnitude higher than that any particle accelerator can achieve. Thus, the required parameters are calculated by extrapolating the parameters from lower energies. Different extrapolations led to different interaction models, such as QGSJET [47], SIBYLL [48], EPOS [49, 50]. These hadronic interaction models vary also on a fundamental level of physics. Details of these hadronic interaction models are beyond the scope of this dissertation. Detailed information on the matter can be found in their respective publications.

The results of different extrapolations of one of the interaction parameters, cross-section, (other interaction parameters are multiplicity, inelasticity, branching ratio) can be seen in

Figure 3.4. Despite the calculation of the same value below $10^{16} \mathrm{eV}$, an uncertainty envelop is formed at higher energies. The envelop is bounded by SIBYLL (in blue) and QGSJET (in black). Detailed comparison of these hadronic interactions is given by Parsons et al. 


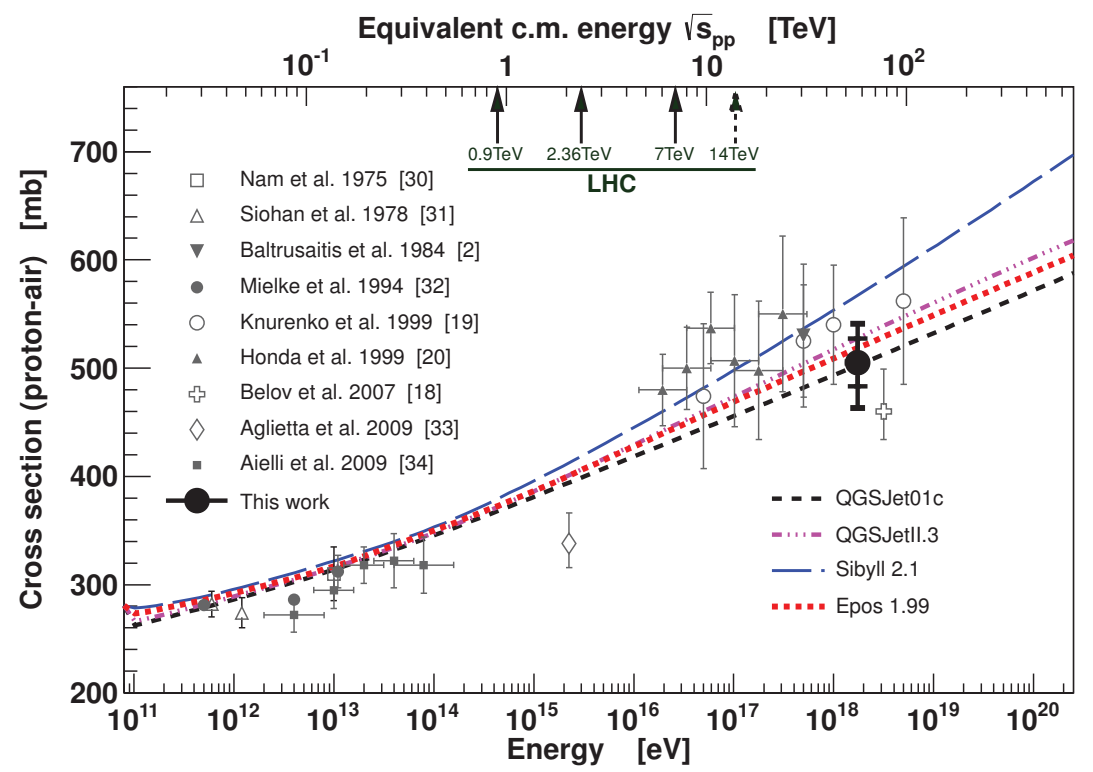

Figure 3.4: Cross-section measurements by different experiments and calculations by different hadronic interaction models [52] 


\section{Chapter 4 The Pierre Auger Observatory}

The detection of ultra high energy cosmic ray events can only be accomplished indirectly due to the low flux of these events. As shown in the energy spectrum of cosmic rays (Figure 2.1), in order to get statistically significant data to answer the questions about cosmic rays, a large detector is necessary. The Pierre Auger Observatory has been built to address the GZK-cutoff phenomena, the arrival direction distribution and mass composition of ultra high energy cosmic rays with high statistical significance over the Southern Sky [53].

The Pierre Auger Observatory is an hybrid observatory spanning $3000 \mathrm{~km}^{2}$ located in Malargue, Argentina. The electromagnetic longitudinal profiles of extensive air showers are observed by 24 fluorescence telescopes located in four sites (Fluorescence Detectors, FDs) along the edges of the observatory. The ground array, called the surface detector, consists of 1600 water Cherenkov detectors. Due to the dependence of FDs to atmospheric conditions, the observatory is also equipped with monitoring instruments such as Weather Stations, Central Laser Facility (CLF), Extreme Laser Facility (XLF) [54].

As stated in earlier chapters, muons carry more information regarding the initial part of the shower development. Thus, this work focuses solely on the surface detector data. Detailed information about the FDs can be found in [55, 56, 57, 58].

\subsection{The Surface Detector}

The surface detector consists of water Cherenkov tanks, with radius of $1.8 \mathrm{~m}$ and height of $1.2 \mathrm{~m}$, filled with 12 tons of pure water. Each tank is equipped with photomultiplier tubes and electronics package including a power controller, radio transceiver, GPS receiver and a processor (see Figure 4.2). For the operation of surface detector, atmospheric conditions 


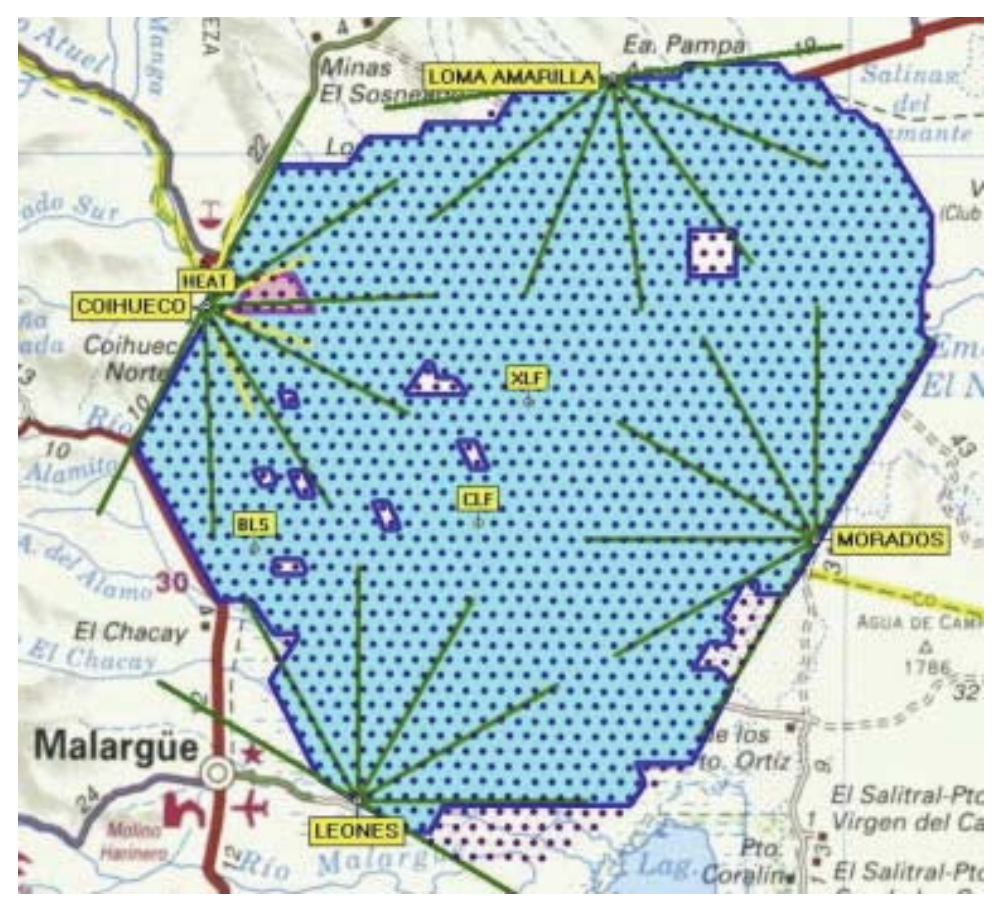

Figure 4.1: Layout of the Pierre Auger Observatory. Dots in the figure represent the water Cherenkov tanks. Four fluorescence detectors each with 6 fluorescence telescopes are located at the edges of the observatory [59]

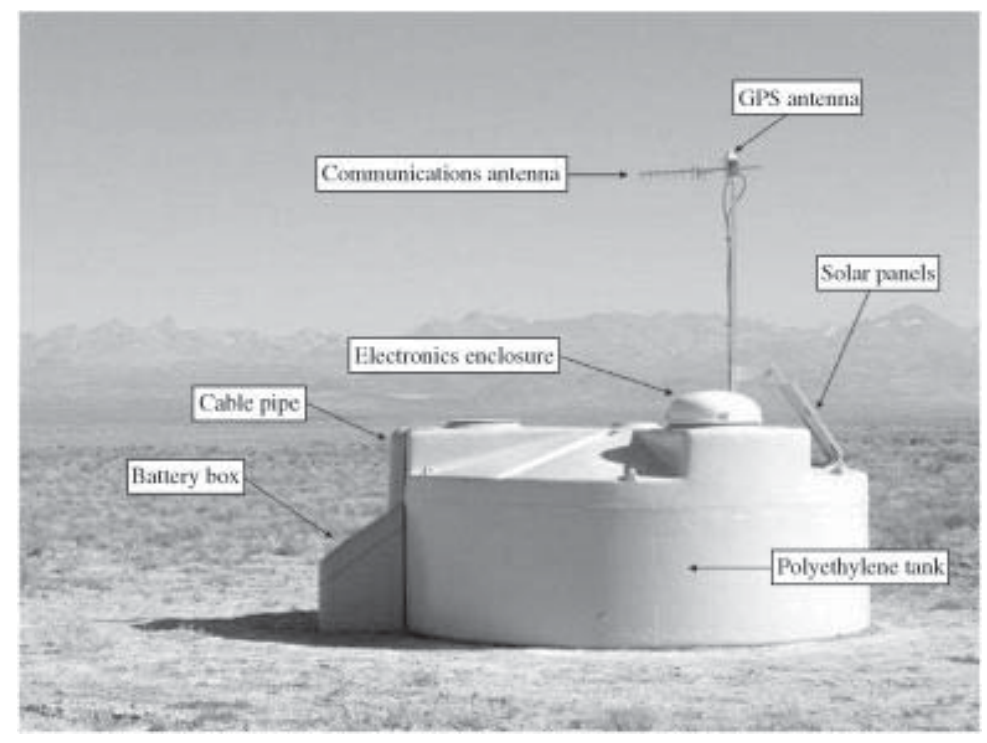

Figure 4.2: A water Cherenkov tank deployed in the Pierre Auger Observatory [60] 
are not important. Unlike the fluorescence detectors, surface detector can function daytime and nights when the moon is up. Thus, the surface detector works at $100 \%$ duty cycle.

The Cherenkov radiation produced as relativistic particles transit the tanks is collected with the three photomultiplier tubes at the top of the tanks. The signals acquired from the tubes are converted into "vertical equivalent muon" (VEM) units (signal of a single muon entering the tank vertically). A sample signal trace is shown in Figure 4.3. To discard the background noise, different levels of triggers were implemented in the software of the processors. Each signal corresponds to a time bin elapsing 25 ns. Technical details concerning the components of the water Cherenkov tanks are given in [60].

Despite all the benefits of the surface detector, it does not measure the energy of the cosmic ray directly. One possible method to measure the energy is to use Monte-Carlo simulations. However, the Monte-Carlo simulations have uncertainties at high energies. The benefit of having a hybrid observatory is that the surface detector can be calibrated using the FDs, since the FDs can measure the energy indirectly by the integration of the electromagnetic profile they observe (see Figure 4.4) [59].

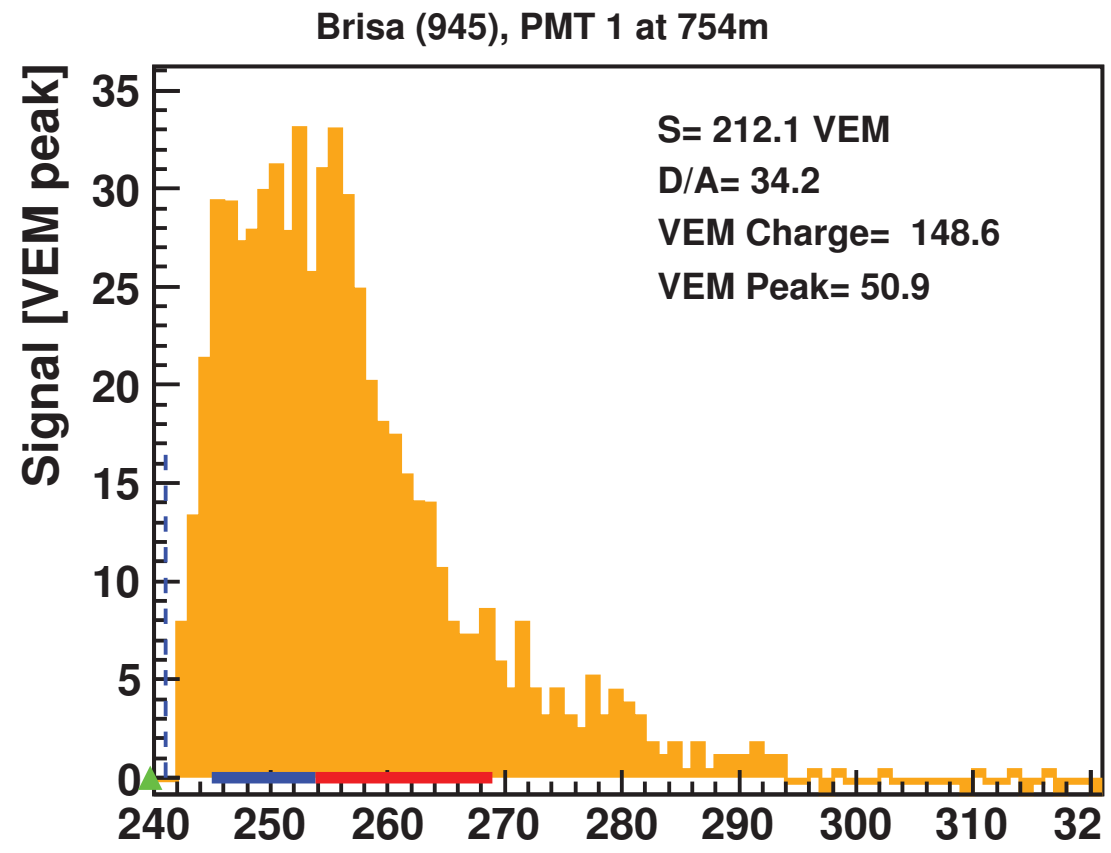

Figure 4.3: A sample signal trace from an observed event 


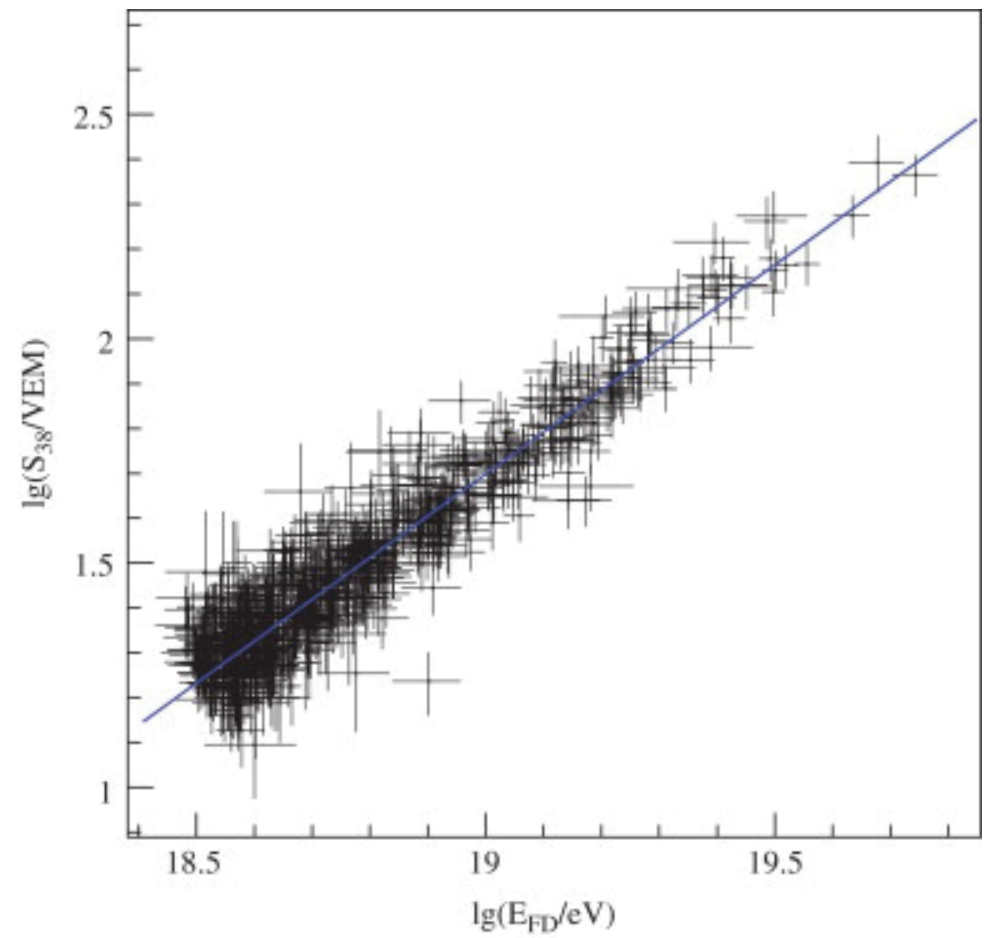

Figure 4.4: Calibration of Surface Detector with Fluorescence Detectors [59]

\subsection{Event Reconstruction}

This section explains the reconstruction process very briefly to give the reader an idea. Any event reconstruction starts with the evaluation of signal traces of the triggered tanks (see Figure 4.5). The integral of the signal for each tank is a measure of the particle density. Using the relative times of the start of the signals, the shower core is found.

The particle density is fitted using a "Lateral Distribution Function" (LDF). The LDF being used in the Pierre Auger Collaboration is shown in Eqn 4.1.

$$
S(r)=S_{1000} \times\left(\frac{r}{1000}\right)^{\beta} \times\left(\frac{r+700}{1700}\right)^{\beta+\gamma}
$$

where $\mathrm{S}(\mathrm{r})$ is the integrated signal of tank $\mathrm{r}$ meters away from the shower core and $\mathrm{S}_{1000}$ is the signal at 1000 meters away from the shower core. $\mathrm{S}_{1000}, \beta$ and $\gamma$ are the free parameters for the fit. To account for the zenith dependence of $S_{1000}$, it is converted to a parameter called $S_{38}$ [59]. Then, $S_{38}$ is used for the calculation of the energy of the event via the calibration with FDs (Figure 4.4). 


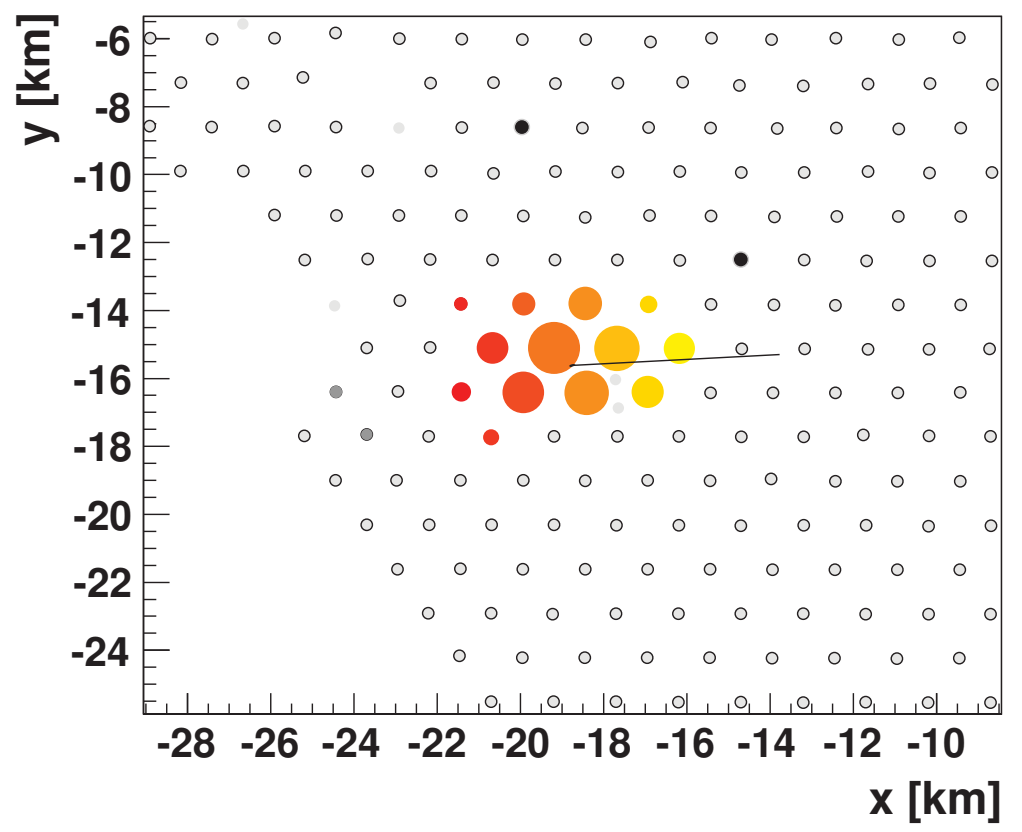

Figure 4.5: Integrated signals in an observed event. Size of the colored circles indicate the integrated signal strength and the color designates the arrival time of the signal.

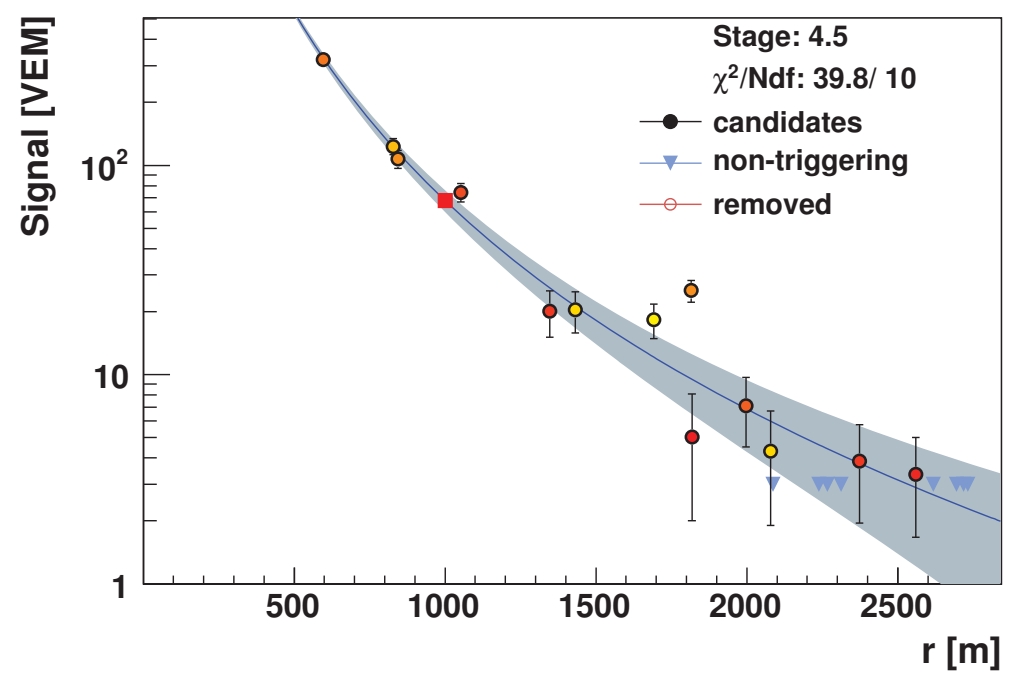

Figure 4.6: LDF fit to integrated signals in an observed event 


\subsubsection{Muon Production Depth (MPD) Profile}

The muon component of the extensive air shower arriving to the ground level exhibits a time structure due to the convolution of production spectra, energy loss, and decay probability during propagation. With some assumptions, the time structure can help to obtain the muon production distributions along the shower axis [61]. A method to transform the time structure (or signal traces) has been developed by the Pierre Auger Collaboration.

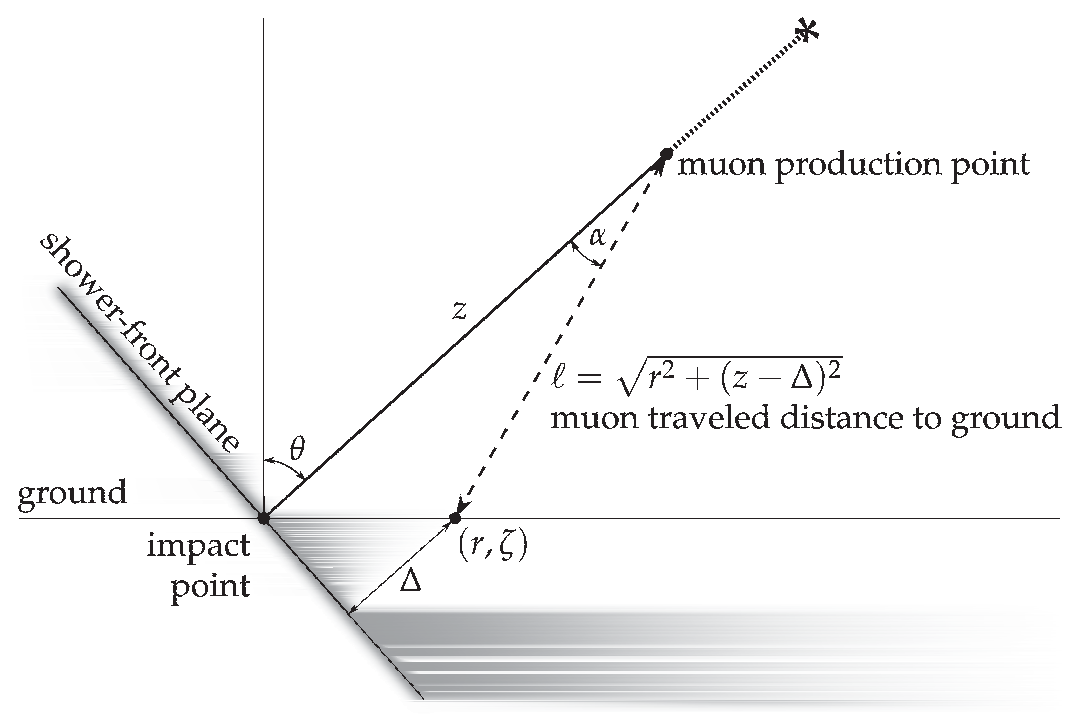

Figure 4.7: Geometric transformation for muon production depth profile [61]

The signal of a detector at a position $(r, \zeta)$ is transformed into a signal of muon production depth profile (for geometry, see Figure 4.7). This delicate transformation is not only a geometric transformation, but it also includes the corrections due to geometric and kinematic delays [62]. The transformation equation is given in Eqn 4.2 [61].

$$
z \simeq\left(\frac{r^{2}}{c\left(t-\left\langle t_{\varepsilon}\right\rangle\right)}-c\left(t-\left\langle t_{\varepsilon}\right\rangle\right)\right)+\Delta-\left\langle z_{\pi}\right\rangle
$$

where $c$ is speed of light, $t$ is the time of signal, $\left\langle t_{\varepsilon}\right\rangle$ is the time delay due to the muon speed being lower than speed of light and $\left\langle z_{\pi}\right\rangle$ is the correction due to the parent particle travel distance.

The discrete number of tanks do not cover $100 \%$ of the ground. Thus, not all the muons at ground level can be used for the method. This constitutes a challenge for the application of 


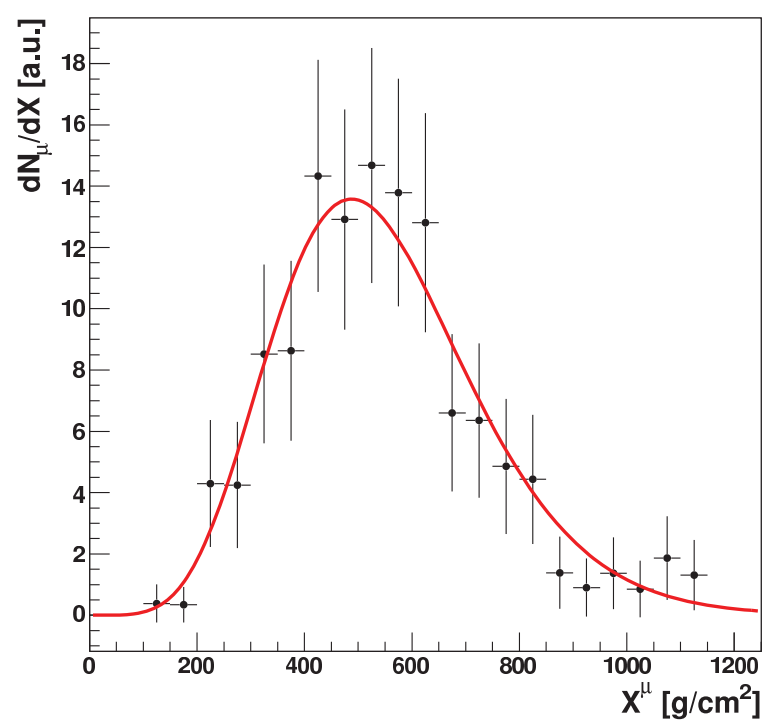

Figure 4.8: A sample reconstructed muon production depth profile [61]

the method. Moreover, a zenith angle constraint had to be introduced due to a dependency formed by the probability of muon decay [61].

Another important factor to be considered is the relative timing of the muons. The signal of the muons close to the core are merged due to time binning at the detectors. This results in high uncertainties for the depth calculations. Thus, a cut for the distance from the core had to be implemented. Under these circumstances, a result of the muon production depth profile is shown in Figure 4.8 [61]. 


\section{Chapter 5 Artificial Neural Networks}

The solution of non-linear problems is challanging whereas linear problems can be solved with methods such as Principle Component Analysis and linear regression analysis. Artificial Neural Networks (ANNs), a branch of machine learning algorithms, are among the methods used for non-linear problem solution . Imitating brain structure, layers of neurons are used to train computers for goals such as decision making and forecasting in various field of science.

In the last two decades, applications of ANNs in particle physics, astrophysics and astronomy have come into prominence. In astrophysics, ANNs have been mostly used for the characterization and the estimation of astrophysical data $[63,64,65,66,67,68]$. ANNs have also been employed for reconstruction of data $[69,70]$.

In cosmic ray physics, multiple applications of ANNs for gamma/hadron separation have been published [71, 72, 73]. Bussino et al. performed ANN application for simulated events that were reconstructed with an ideal detector [71]. Bionee et al. incorporated Self Organizing Tree Algorithm and ANNs for MAGIC Cherenkov Telescope [72]. Likewise, Sharma et al. tested various machine learning algorithms including Artificial Neural Net-

works for segregation of gamma and hadrons for Cherenkov Telescopes [73]. In addition to gamma/hadron seperation, of particular importance, Tiba et al. has incorporated Principle Component Analysis (PCA) with Neural Networks for distinguishing proton and iron cosmic rays detected by Pierre Auger Observatory [74].

The ability of ANNs to make decisions also makes them a good tool for electronics software. ANNs were employed in electronics software for setting triggers [75, 76] 


\subsection{Morphology of Artificial Neural Networks}

Neural Networks consist of characteristic inputs, neurons, weights assigned for each inputneuron interaction and output(s). Characteristic inputs are fed to neurons with their weights to yield output(s).

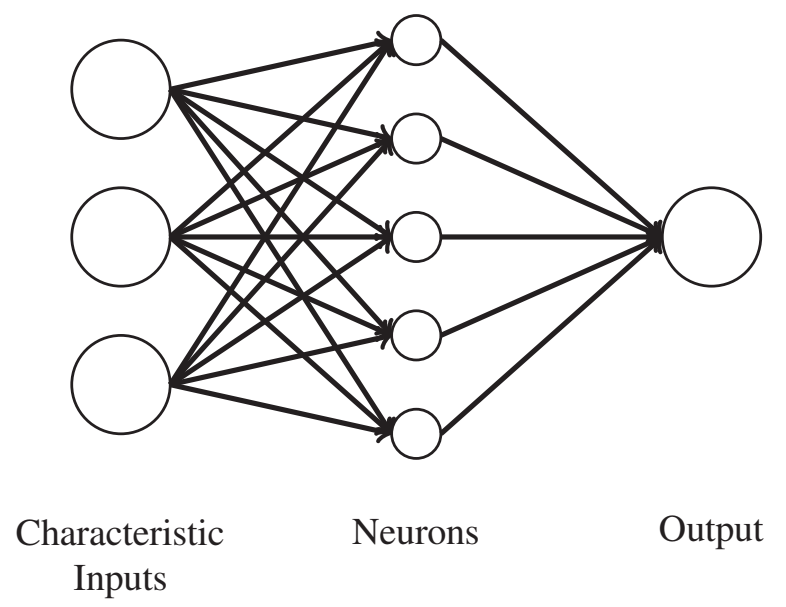

Figure 5.1: Illustration of an ANN. Three characteristics input are chosen with a single layer of 5 neurons to characterize a single output.

At each neuron, weighted inputs pass through an activation junction (Fig. 5.1). In more simplistic terms, for a single layered ANN with single output can be described as the following function:

$$
y=\sum_{i=1}^{N_{i}} f\left(\sum_{j=1}^{m} \theta_{i j} x_{j}\right)
$$

where $y$ is the output of the ANN, $N_{i}$ is the number of neurons in the layer, $m$ is the number of characteristic inputs, $\theta_{i j}$ is the weight of $\mathrm{j}^{\text {th }}$ characteristic input for $\mathrm{i}^{\mathrm{t}}$ neuron and $f(\cdot)$ is an activation function. The most used activation functions are step function, linear function, sigmoid function and tan-sigmoid function.

Even though the method is an imitation of brain neural structure, it is still an optimization method. Because of the number of characteristic inputs and the number of neurons, the number of weights to be optimized creates a large optimization space. Depending on the algorithm chosen, an ANN may be optimized to a local minimum [77]. Thus, any user 
should be cautious about the solution for a given case. Some possible solutions suggested to avoid local minima are repetitive training with randomized weights and adding extra hidden units [77].

The results of an ANN can also be interpreted to understand the importance of the characteristic inputs [77]. A lower weight for a characteristic input does not carry much information that contributes to the overall result. Thus, the lower the weight associated with a given characteristic input, the less significant the characteristic input is. This not only decreases the computation time at the end, but also decreases the potential uncertainty of the output(s) propagated by many characteristic inputs.

\subsection{Artificial Neural Network Software packages}

There are available packages for different software. One of the available packages is provided by MATLAB. For this work, we have used the MLPF it package provided by ROOT [78] due to its compatibility with other data analysis codes written. 


\section{Chapter 6 Developing a First Interaction Length Prediction Model}

\subsection{Searching for proxies of first interaction lengths}

The extensive air shower development depends on the primary particle initiating the shower. Combined with the large variations of elasticity and multiplicity, the probability distribution of the first interaction length is one of the main reason for the large fluctuation in shower observables [79].

Limited to a single primary particle spcies, the Pierre Auger Collaboration incorporated the distribution of $\mathrm{X}_{\max }$, in order to calculate the p-air cross-section [52]. However, the result was not extended to higher energies due to possible changes of cosmic ray composition.

Muons that are the products of the decay of mesons transmit more information to the ground level about the cosmic rays. Thus, it can be concluded that the muonic shower profile carries critical information about cosmic rays. For the characterization of cosmic rays, different approaches have been reported such as the search for a single parameter extracted from a muon production profiles and total muon production profile. Some of these studies are explained below.

The number of muons at ground level, $\mathrm{N}_{\mu}$, was found to be the one of the promising parameters for cosmic ray primary particle identification [80, 81, 82, 83]. Although the reported works were focused on identification, they did not address the properties of the particle such as cross-section. KASCADE-Grande experiment, incorporating muon tracking detectors, investigated the mean muon production height, $\left\langle\mathrm{h}_{\mu}\right\rangle$ [84]. Being capable of detecting muons separately from the electromagnetic part of the shower, they demonstrated the relationship between mean muon production height and the total number of 
muons generated for light and heavy primary particles for vertical showers [84]. Yet this study also did not address the characteristic properties of the primary particles.

To serve the purpose for both the identification of the primary particle and its characteristic properties, Andringa et al. investigated two shower profile shape parameters, $\mathrm{L}_{\mu}$ and $\mathrm{R}_{\mu}$ [85]. These shape parameters were derived from the Gaisser-Hillas profile shifted by shower maximum. Parameters were examined with respect to $\Delta \mathrm{X}$, which indicates the fluctuations after the first interaction point. These parameters were defined as:

$$
\begin{aligned}
\mathrm{L}_{\mu} & =\lambda_{\mu} \times \mathrm{X}_{\mu, 0} \\
\mathrm{R}_{\mu} & =\frac{\lambda_{\mu}}{\mathrm{X}_{\mu, 0}} \\
\Delta \mathrm{X}_{1} & =\mathrm{X}_{\mu, \max }-X_{\mu, 1}
\end{aligned}
$$

where $\lambda_{\mu} . \mathrm{X}_{\mu, 0}$ and $\mathrm{X}_{\mu, \max }$ are parameters obtained through Gaisser-Hillas function fit to muon production depth profile of a single shower. The event-by-event based resolution of this work has been reported to be of the order of $50 \mathrm{~g} / \mathrm{cm}^{2}$ [85].

Due to the exponential increase in the number of secondaries in the EAS, it was thought that $\mathrm{X} 37$, which is the inverse of the difference of $\mathrm{X}_{\mu, \max }$ and slant depth at which the ratio of the number of muons produced is $37 \%$ of the number of muons produced at $\mathrm{X}_{\mu, \max }$ would yield a proxy for the first interaction length. Despite showing composition independence to some extent, X37 did not yield hadronic interaction independence (Figure 6.2). Several other parameters, in conjunction with the characteristics of the shower development such as X37, were also investigated. However, it was evident that an approach using the shape of the shower profile rather than a single parameter was needed. Being complicated to find the link between the shape of the profile and the first interaction length, we focused on a rather complicated method, Artificial Neural Networks, to identify the parameters needed to be connected to first interaction length. 

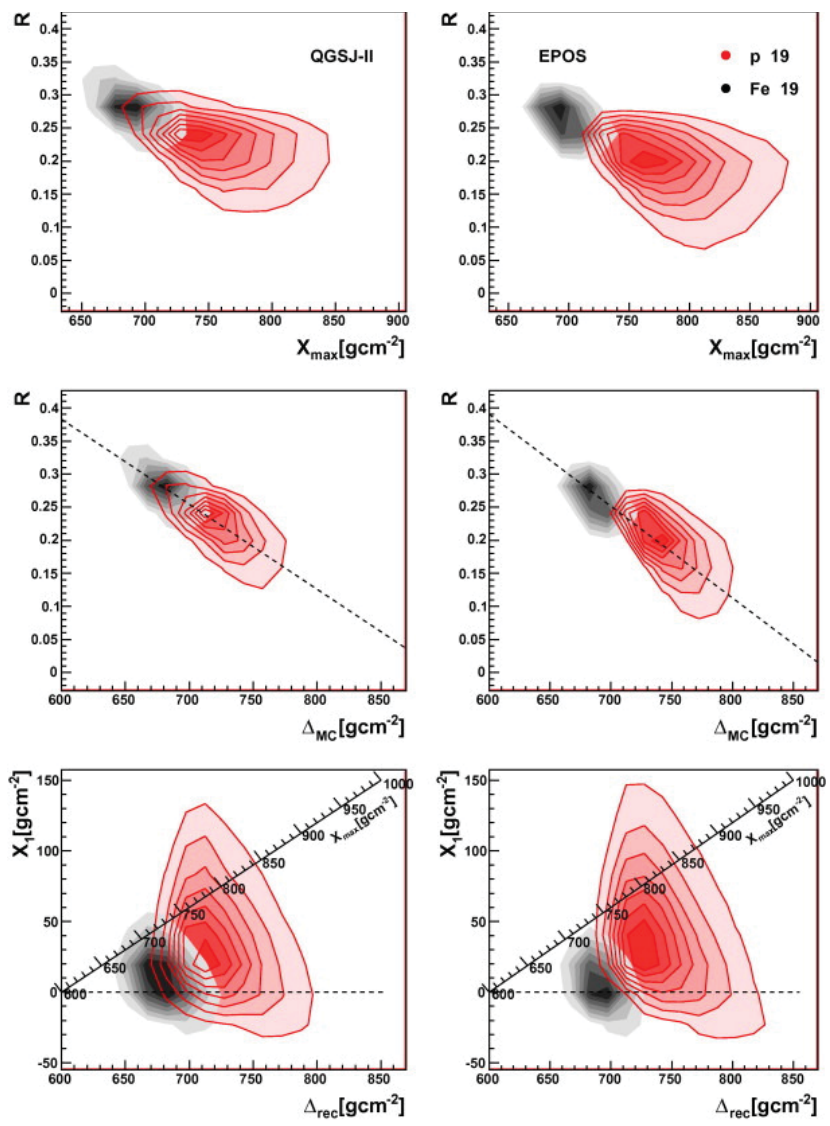

Figure 6.1: Plots for reconstructed $R, X_{\max }$ and $X_{1}$ values for iron and proton showers at $10^{19} \mathrm{eV}$. Left column of plots correspond to the showers generated by QGSJETII model and right columns of plots correspond to the showers generated by EPOS model.[85]

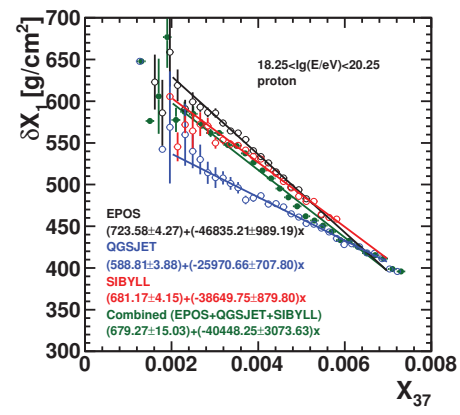

(a)

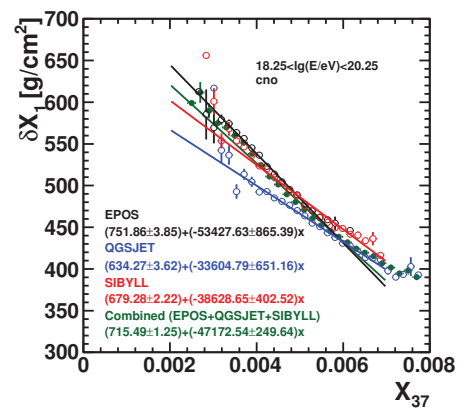

(b)

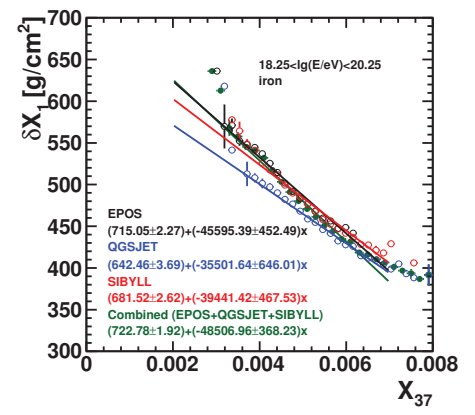

(c)

Figure 6.2: Profile plot of $\delta \mathrm{X}$ with respect to X37 and $1^{\text {st }}$ order polynomial fit for showers generated by different hadronic interaction models (a) for proton, (b) for CNO, (c) for iron. 


\subsection{Artificial Neural Network based Prediction Model}

Artificial Neural Networks (ANNs) are excellent tools for non-linear problems. For this work, ANNs were exercised for two purposes, decision making and prediction.

\subsubsection{Training and Testing of Artificial Neural Network based prediction model}

Shower development is initiated at high altitudes (or small slant depths) and the exponentially increasing number of particles transverse the atmosphere. As the number of particles increase, their energies gets smaller such that after a depth in the shower development the decay/absorption of particles dominate. Despite many hadronic interactions along the longitudinal development, the most significant ones that decide on the shape of the shower profile are the first few interactions [86]. Thus, the required information for training an ANN is encrypted in the rising part of the longitudinal muonic profile. In the light of this fact, several characteristic inputs were generated and tested. Even though the longitudinal muonic profile has more information than the longitudinal electromagnetic profile for the reasons mentioned in Chapter 3, characteristic inputs computed from EM profile were also considered to test the validity of the statement. The list of these characteristic inputs is given in Table 6.1 with their explanations. $\theta=40$ was drawn as an example in Figure 6.3.

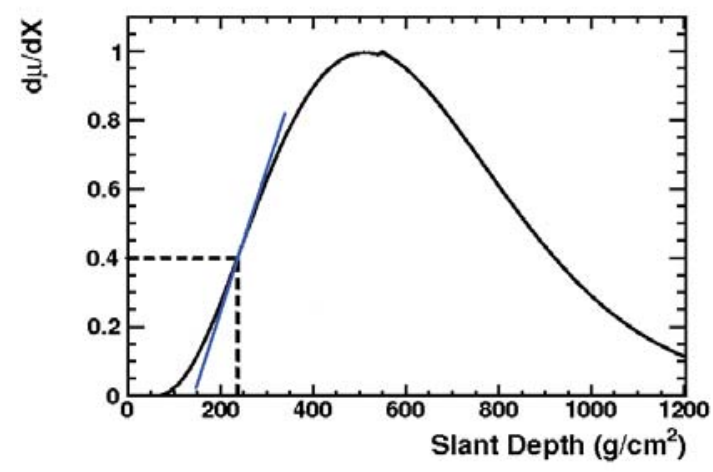

Figure 6.3: A sample shower profile with $\theta=40$ for illustration. The vertical dashed line indicates where $\mathrm{X} 40_{\mu}$ is. $\left.\mathrm{d} \mu_{x}\right|_{X 40}$ is the slope of the profile. A blue line was drawn to guide. 
Table 6.1: List of characteristic inputs tested

\begin{tabular}{|c|c|}
\hline $\bar{X} 10_{e m}$ & $\begin{array}{l}\text { Slant depth difference between the EM shower profile maximum } \\
\text { and the depth at which the EM shower profile is at its } 10 \%\left(\mathrm{X} 10_{e m}\right)\end{array}$ \\
\hline$\left.\mathrm{dE}_{x}\right|_{X 10}$ & Slope of EM profile at $\mathrm{X} 10_{e m}$ \\
\hline $\begin{array}{r}\bar{X} \theta_{e m} \\
(\theta=20,30,40,50)\end{array}$ & $\begin{array}{l}\text { Slant depth difference between the EM shower profile maximum } \\
\text { and the depth at which the EM shower profile is at its } \theta \%\left(\mathrm{X} \theta_{e m}\right)\end{array}$ \\
\hline $\begin{array}{r}\left.\mathrm{dE}_{x}\right|_{X \theta} \\
(\theta=20,30,40,50)\end{array}$ & Slope of EM profile at $\mathrm{X} \theta_{e m}$ \\
\hline $\bar{X} 10_{\mu}$ & $\begin{array}{l}\text { Slant depth difference between the muonic shower profile maximum } \\
\text { and the depth at which the muonic shower profile is at its } 10 \%\left(\mathrm{X} 10_{\mu}\right)\end{array}$ \\
\hline$\left.\mathrm{d} \mu_{x}\right|_{X 10}$ & Slope of muon production profile at $\mathrm{X} 10_{e m}$ \\
\hline $\begin{array}{r}\bar{X} \theta_{\mu} \\
(\theta=20,30,40,50)\end{array}$ & $\begin{array}{l}\text { Slant depth difference between the muonic shower profile maximum } \\
\text { and the depth at which the muonic shower profile is at its } \theta \%\left(\mathrm{X} \theta_{\mu}\right)\end{array}$ \\
\hline $\begin{array}{r}\left.\mathrm{d} \mu_{x}\right|_{X \theta} \\
(\theta=20,30,40,50)\end{array}$ & Slope of muon production profile at $\mathrm{X} \theta_{e m}$ \\
\hline $\mathrm{P} 26$ & $\mathrm{X} 10-\mathrm{X} 20$ \\
\hline P27 & $\mathrm{X} 20-\mathrm{X} 30$ \\
\hline $\mathrm{Xd} \mu \mathrm{mx}$ & muonic shower profile maximum \\
\hline
\end{tabular}

The ANN was trained for different hadronic interaction models to observe their individual dependencies; however, at the end, with our current knowledge, there is no way of knowing which hadronic interaction works properly at high energies. Thus, training needed to be done in such a way that even if none of the hadronic interaction models is correct, the ANN should be able to decipher the first interaction length information. It was not possible to feed the ANN with a known incorrect interaction model, but it was possible to feed it with the combination of showers generated by three hadronic interaction models. By doing this, an effective hadronic interaction model would be fed to the ANN, possibly removing any possible interaction model biases.

No hadronic interaction model is absolutely correct; likewise, the primary particle impinging the atmosphere is not known with certainty. Thusly, training an ANN with showers generated by only a single primary particle would result in biases for the showers generated by others. In this manner, because of the possible cosmic source scenarios mentioned in Chapter 2.4, the combination of showers generated by different primary particles were fed to ANN. These primaries, proton, carbon, nitrogen, oxygen and iron, should provide sufficient information at various cross-sections, multiplicity and elasticity for the ANN to interpolate/extrapolate any other known/unknown primary. 
One of the important aspects of the ANNs is their ability to train and in some cases to memorize. When trained, an ANN can be used for other datasets; however, when ANNs memorize, they do not work efficiently for other datasets. In order to avoid any memorization, the mentioned shower database was divided into two subsets. One of the subsets was used to train and the other half was used for validation. During the training of the ANN, the errors of first interaction length on the validation dataset were tracked. When the errors on the validation dataset started increasing, training was terminated to stop memorization.

An important feature about constructing the training and validation datasets is to remove the bias due to the first interaction length distribution. Since the probability of getting a smaller interaction length is higher, clustered small interaction lengths pull the learning curve towards themselves. This results in high errors at bigger interaction lengths. Thus, showers from the generated datasets were selected using a filter that attempted to flatten the distribution going into the training process. It is worth noting that the first interaction lengths are calculated by Monte-Carlo number generators in hadronic interaction models. Thus it was not possible to obtain a flat distribution.

The initial ANN was constructed with 36 neurons on a single hidden layer. For the activation function, a sigmoid function was used. To account for the scale of the characteristic inputs, each characteristic input was normalized to range in $[0,1]$.

For the ANN to achieve a global minimum as mentioned in Chapter 5, the ANN was trained with different initial weights multiple times. The results of the training for three different interaction models and the effective hadronic interaction model with a combination of three primaries (proton, $\mathrm{CNO}$ and iron) are used for decision making to limit the number of characteristic inputs (Fig. 6.4).

Showers generated by QGSJET and EPOS interaction models exhibited high dependency on muonic shower parameters. Despite the dependency of EPOS on muonic shower parameters, the ANNs also display a dependence on a certain parameter on EM shower development. A similar trend was exhibited for SIBYLL except for $4^{\text {th }}$ trial. A possible explanation was ANNs tending to EM shower development ended up in one of the local minima. This hypothesis was supported when ANNs were trained with combined shower set. 
Table 6.2: List of selected characteristic inputs

\begin{tabular}{|r|l|}
\hline $\bar{X} 10_{\mu}$ & $\begin{array}{l}\text { Slant depth difference between the muonic shower profile maximum } \\
\text { and the depth at which the muonic shower profile is at its } 10 \%\left(\mathrm{X} 10_{\mu}\right)\end{array}$ \\
\hline$\left.\mathrm{d} \mu_{x}\right|_{X 10}$ & $\mathrm{~d} \mu / \mathrm{dX}$ at $\mathrm{X} 10_{e m}$ \\
\hline $\mathrm{P} 26$ & $\mathrm{X} 10-\mathrm{X} 20$ \\
\hline $\mathrm{P} 27$ & $\mathrm{X} 20-\mathrm{X} 30$ \\
\hline $\bar{X} 37_{\mu}$ & $\begin{array}{l}\text { slant depth difference between muonic shower profile maximum and the depth at } \\
\text { which the muonic shower profile is at its } 37 \%\end{array}$ \\
\hline $1 / \mathrm{X} 37_{\mu}$ & Inverse of $\mathrm{X} 37_{\mu}$ \\
\hline $\mathrm{X} \mathrm{d} \mu \mathrm{mx}$ & Slant depth of muonic shower profile maximum \\
\hline
\end{tabular}

Having established the basis for ANN, we have decided to decrease the number of characteristic inputs in the light of Fig. 6.4. In addition, another parameter $(\mathrm{Xd} \mu \mathrm{mx})$ was included in the search space to introduce the energy dependence. The results are shown in Fig. 6.5.

The inclusion of $\mathrm{Xd} \mu \mathrm{mx}$ shifted the leaning of weights to itself; that is the energies of the showers contribute significantly in ANN learning. Due to our knowledge that muonic shower development carries more information and the ANN weight analysis, The EM shower development has been removed from the analysis and all steps were repeated. Afterwards, the parameters listed in Table 6.2 were chosen. Then, the number of neurons and number of hidden layers were experimented with to yield the least error in predictions. The structure was finalized with one hidden layer with 100 neurons. 

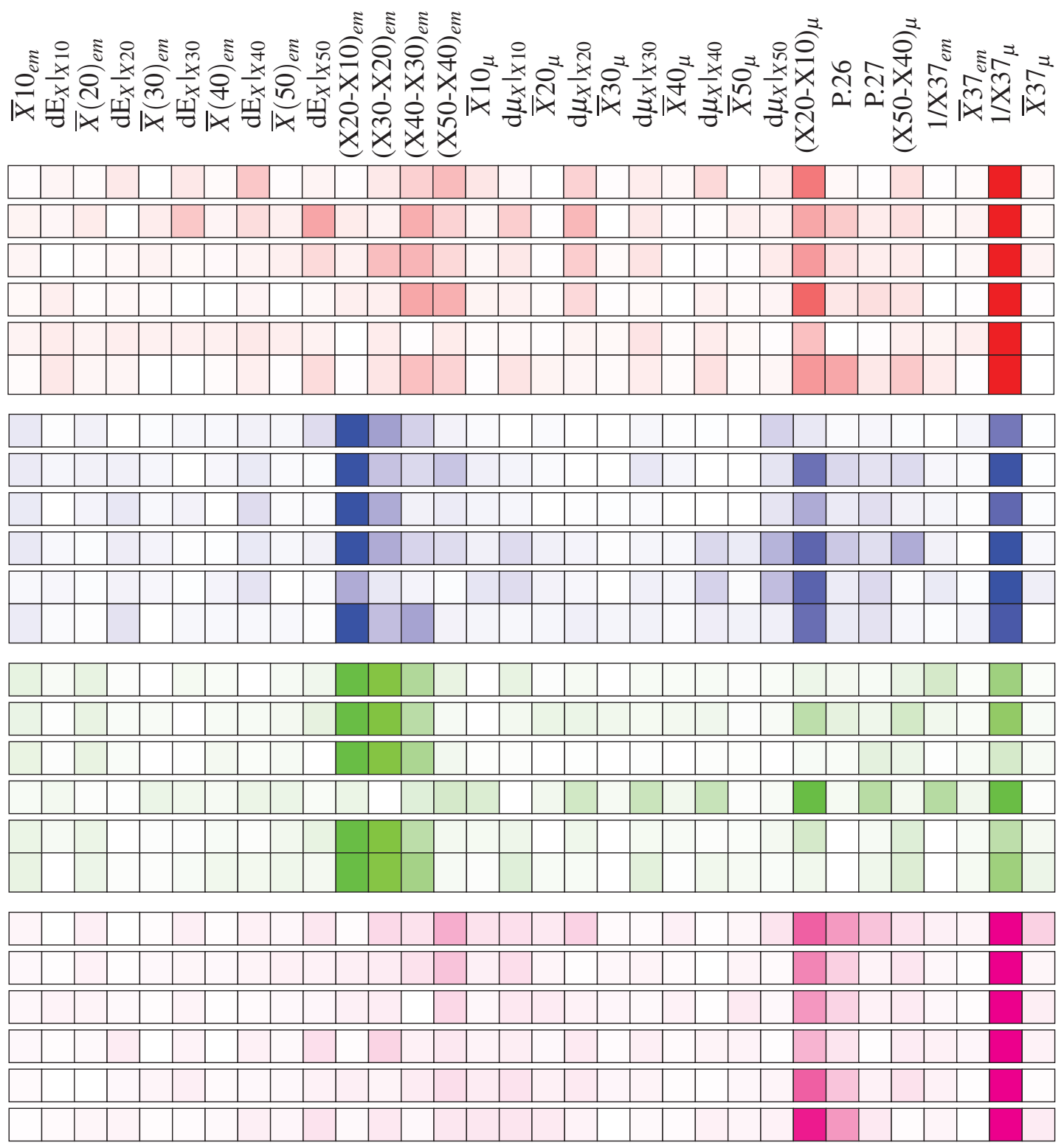

Figure 6.4: Diagram of weights for different hadronic interaction models, QGSJET in red, EPOS in blue and SIBYLL in green. Results of the training after combining the showers from three hadronic interaction models is shown in magenta. Darker colors indicate higher weights. 

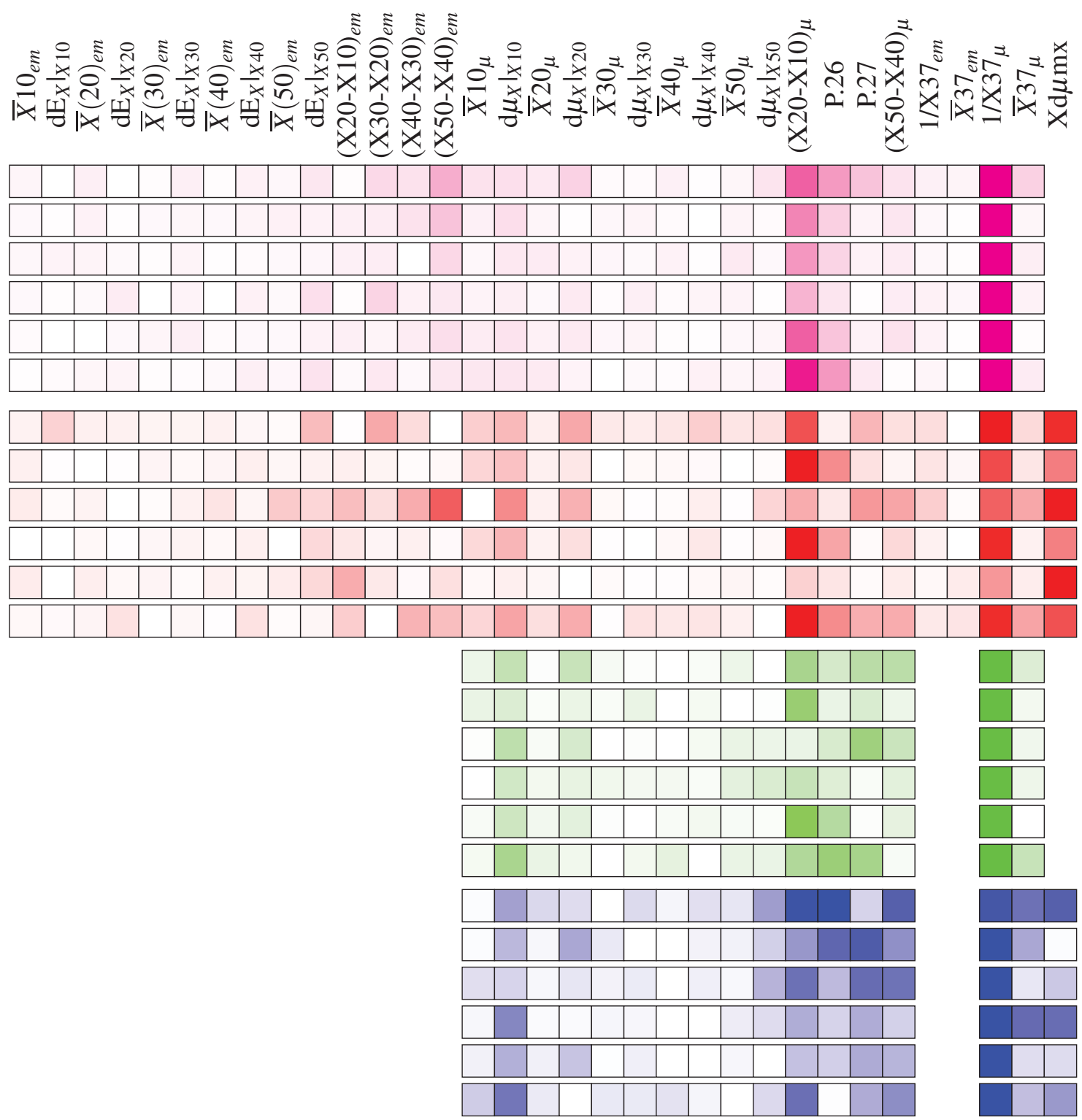

Figure 6.5: Diagram showing the influence of adding $\mathrm{Xd} \mu \mathrm{mx}$ to the characteristic inputs for showers of combination of three hadronic interaction model. Bottom two blocks (green and blue) show the weights for training in the absence of the electromagnetic components' information. Darker colors indicate higher weights. 


\subsubsection{Testing the Prediction Model based on Artificial Neural Network (pmanN)}

The prediction model based on ANN (pmANN) was investigated thoroughly for individual interaction models, different energies and primary particle (See Figs. 6.6-6.22).

It was observed that at low interaction lengths (Figs. 6.6, 6.8, 6.10, 6.12, 6.14, 6.16, $6.18,6.20,6.22)$, the relative error between the predictions and the simulations was high ( $\sim 100-200 \%)$. These relative error values correspond to a maximum of $\sim 15 \mathrm{~g} / \mathrm{cm}^{2}$ error at $\mathrm{X}_{1, \operatorname{sim}} \sim 15 \mathrm{~g} / \mathrm{cm}^{2}$. Thus, only predictions greater than $15 \mathrm{~g} / \mathrm{cm}^{2}$ were considered for interpretation of the results for mean free path and other further analysis.

\subsubsection{Determining Uncertainties with Bootstrap Method}

The bootstrap is a method, developed over two centuries ago, to determine the statistical confidence intervals of data sets [87]. Another use of the bootstrap is for calculating (random) uncertainties of a value for given dataset $[88,89]$. Being a very simplistic computer-based statistical method, it provides insight about the intrinsic variations [87]. Method relies on producing new samples from a given sample set by re-sampling with replacement [89].

The general procedure of the bootstrap method employed for uncertainty determination is as follows. A given set of measured (or computed) quantities constructs the vector $\mathbf{X}=\left(\mathrm{X}^{1}\right.$, $\left.\mathrm{X}^{2}, \mathrm{X}^{3}, \ldots, \mathrm{X}^{N}\right)$ where superscripts indicate indices and $N$ is the number of measurements. At each repetition of the bootstrap method, randomly chosen elements in $\mathbf{X}$ are replaced with another element, creating $\mathbf{X}^{*}=\left(\mathrm{X}^{1 *}, \mathrm{X}^{2 *}, \mathrm{X}^{3 *}, \ldots, \mathrm{X}^{N *}\right)$. At each repetition, the mean value of the set $\mathbf{X}^{*}$ can be computed by fitting an appropriate distribution function. Thus, for each repetition a set, $\left(\mathbf{X}^{*}, \lambda^{*}\right)_{i}$, is constructed where $i$ indicates the repetition index and $\overline{\mathrm{X}_{i}^{*}}$ is the mean value of the set $\mathbf{X}_{i}^{*}$. The distribution constructed by the mean values of the set, $\lambda_{i}^{*}$ at each repetition will yield the expected mean value, $\langle\lambda\rangle$, and the uncertainty, $\sigma_{\lambda}$, of $\mathbf{X}$.

For an event-by-event based analysis, in order to determine the uncertainty in predictions, we have employed the bootstrap method. Given the size of the shower dataset, the vector $\mathbf{X}$ was constructed with $\mathrm{X}_{1}$ values. Multiple repetitions were completed with random placement. An exponential distribution ( $\mathrm{Sec} 4.2 .1)$ was fit to each repetition and the mean 
free path values were recorded. Then, through the distribution of the mean free paths, the expected mean free path values and their uncertainties were calculated. The results for different hadronic interactions were given in the following sections.

\subsubsection{Determining model input values for mean free path}

The evaluation of the predicted values by pmANN requires the hadronic interaction model input values. In order to find the mean free paths of the primaries in consideration for the hadronic interaction models, the first interaction lengths from the simulated showers were used incorporating bootstrap method.

At four energy bins $(\log (\mathrm{E} / \mathrm{eV})=18.75,19.00,19.50$. 20.00), for a hadronic interaction model and a primary particle, the first interaction length values were used to construct the $\mathbf{X}$ vector explained in 6.2.2.1. After the repetitions, the mean free paths were obtained. The results are tabulated in Table 6.3.

\subsubsection{Testing PmANN with simulated showers}

The distributions of mean-free paths, $\lambda$ 's, for QGSJET are shown in 6.7, 6.9, 6.11 at four different energy bins, $\log (\mathrm{E} / \mathrm{eV})=18.75,19.00,19.50$ and 20.00 for proton, CNO and iron, respectively. The number of showers used for each primary particle in each energy bin are tabulated in Table 6.4.

The procedure was also applied to SIBYLL and EPOS shower simulations. The distributions of mean-free paths, $\lambda$ 's, for SIBYLL were shown in $6.13,6.15,6.17$ at four different energy bins, $\log (\mathrm{E} / \mathrm{eV})=18.75,19.00,19.50$ and 20.00 for proton, CNO and iron, respectively and the expected values of the mean-free paths were tabulated in Table 6.4. The distributions of mean-free paths, $\lambda$ 's, for EPOS were shown in 6.19, 6.21, 6.23 at four different energy bins, $\log (\mathrm{E} / \mathrm{eV})=18.75,19.00,19.50$ and 20.00 for proton, $\mathrm{CNO}$ and iron, respectively and the expected mean-free path values were tabulated in Table 6.4. 


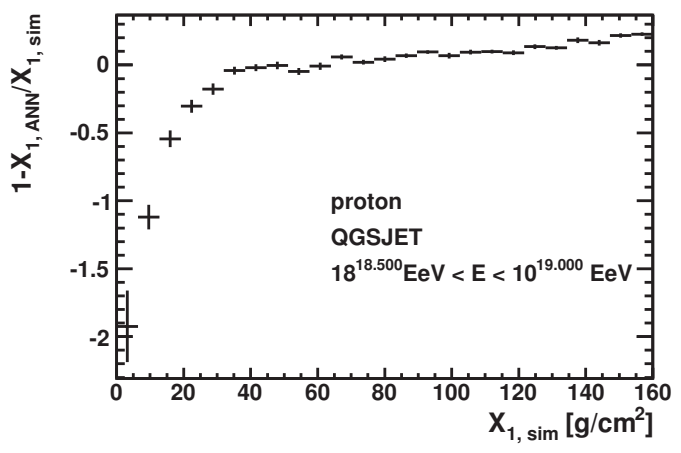

(a)

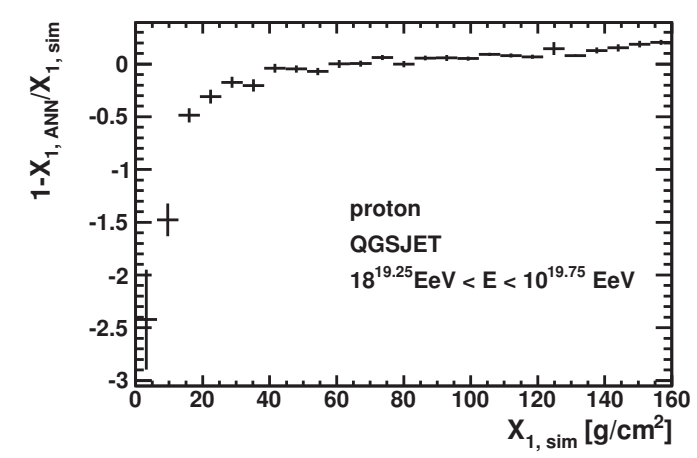

(c)

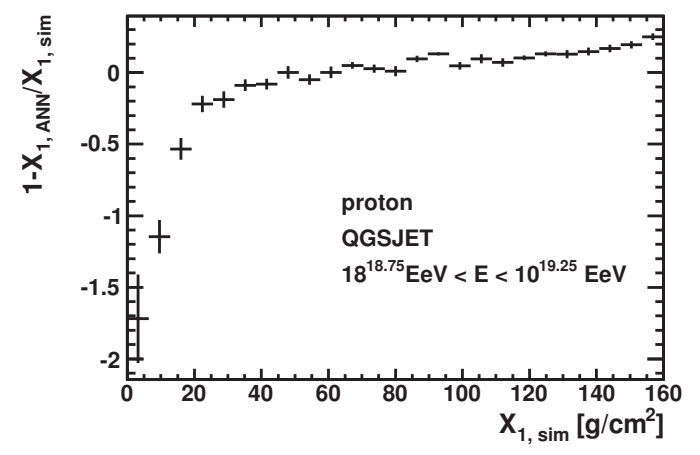

(b)

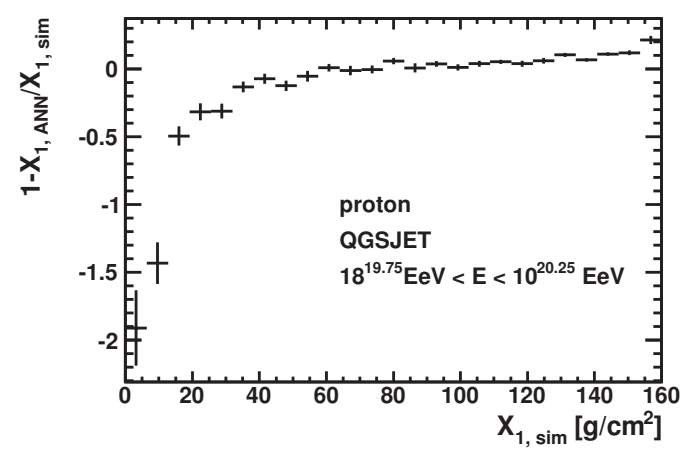

(d)

Figure 6.6: Relative difference between the simulation values and ANN prediction for different energy bins of QGSJET proton showers. 


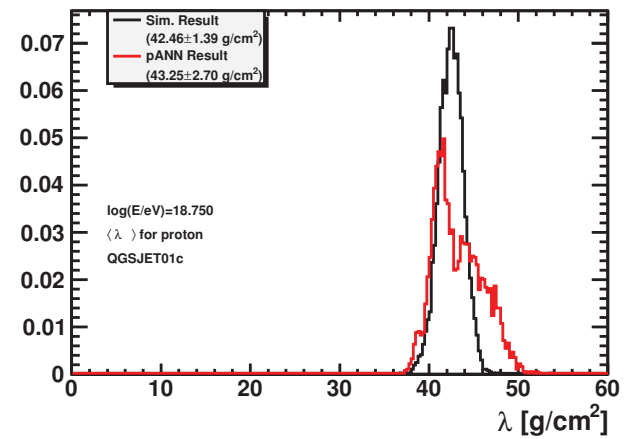

(a)

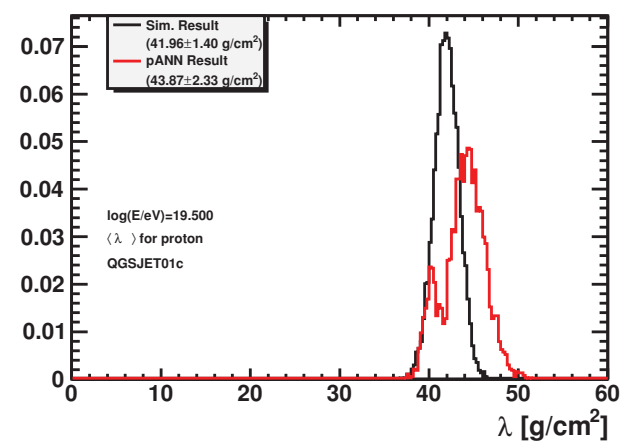

(c)

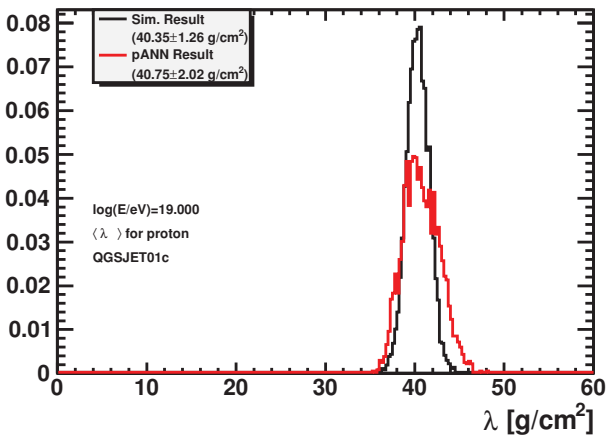

(b)

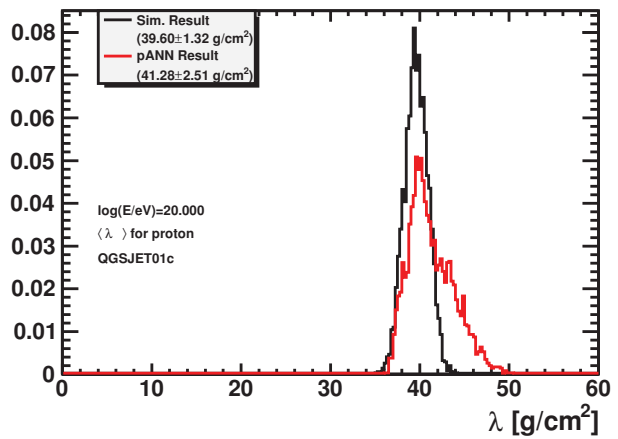

(d)

Figure 6.7: Distribution of mean-free paths constructed by bootstrap method for proton showers generated by QGSJET hadronic interaction model at four different energy bins 


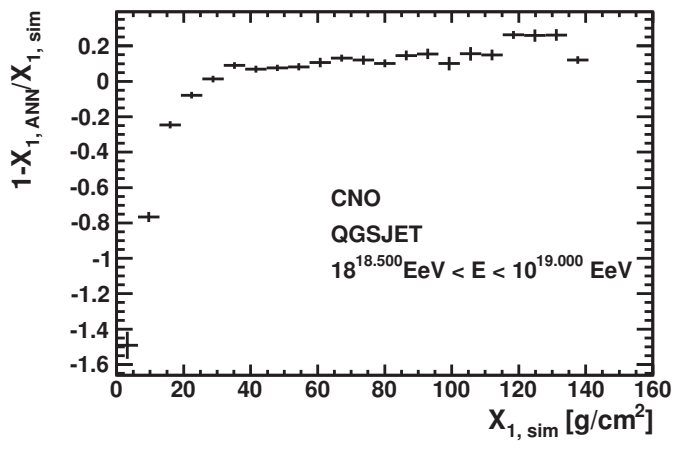

(a)

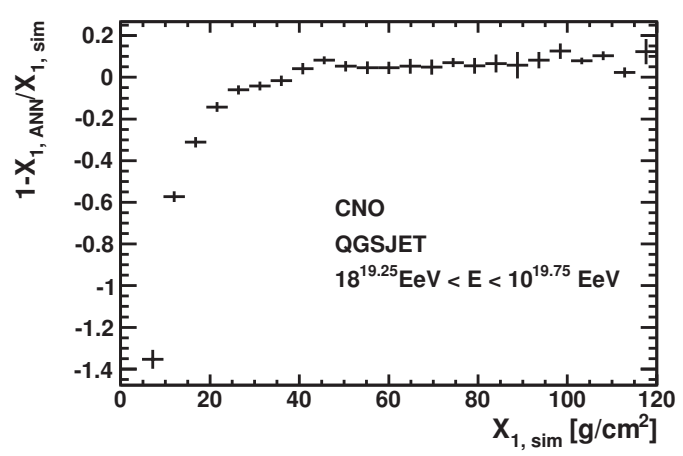

(c)

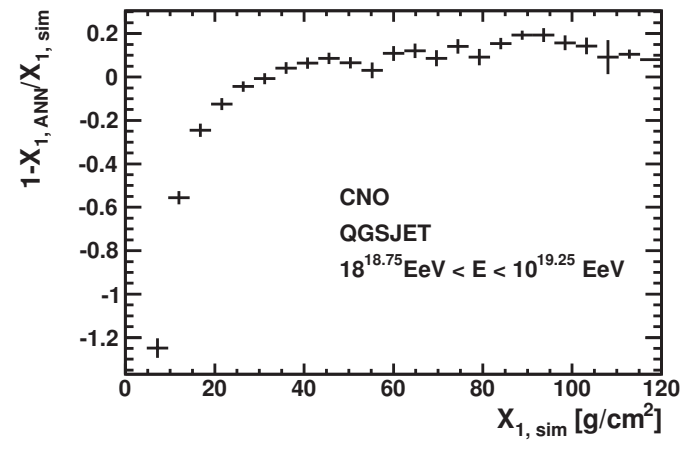

(b)

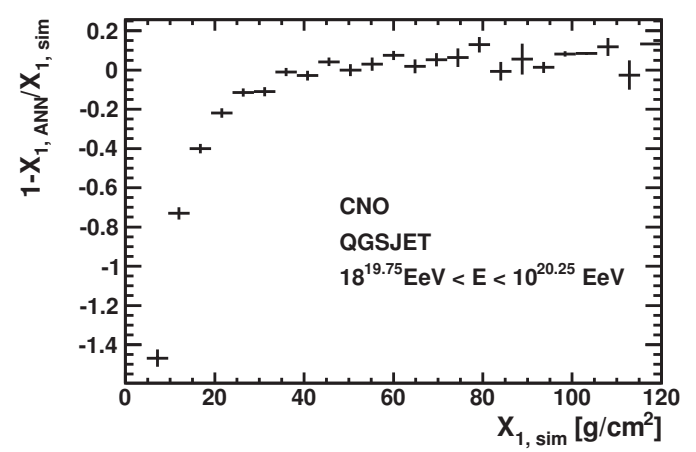

(d)

Figure 6.8: Relative difference between the simulation values and ANN prediction for different energy bins of CNO showers. 


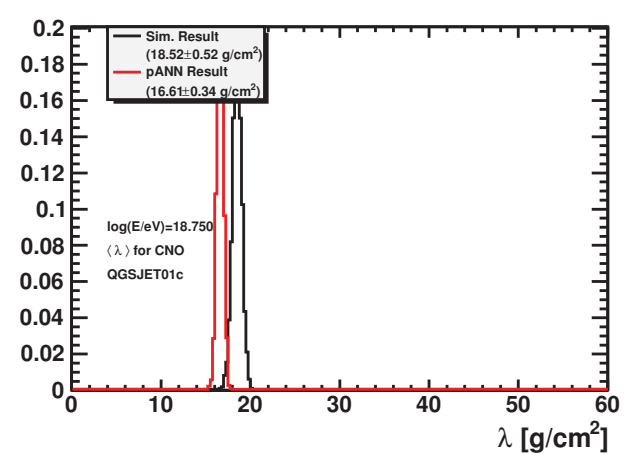

(a)

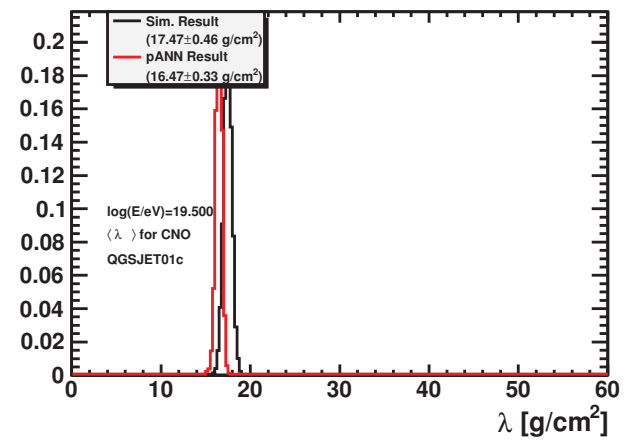

(c)

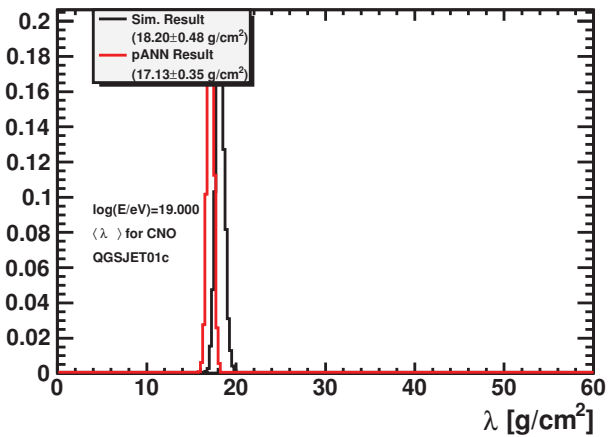

(b)

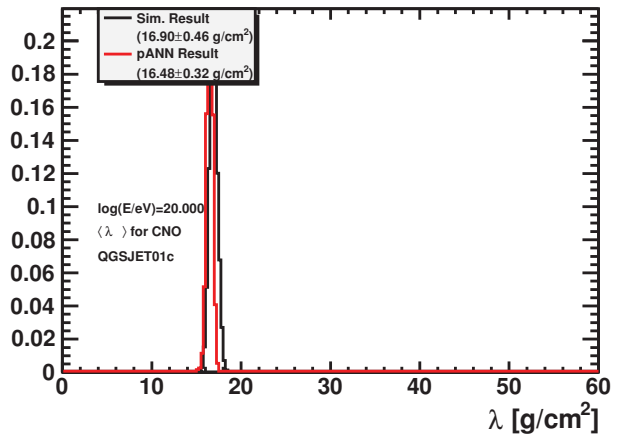

(d)

Figure 6.9: Distribution of mean-free paths constructed by bootstrap method for CNO showers generated by QGSJET hadronic interaction model at four different energy bins 


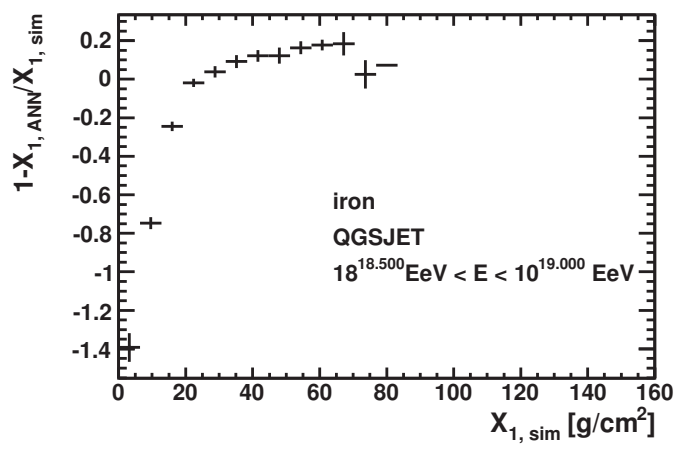

(a)

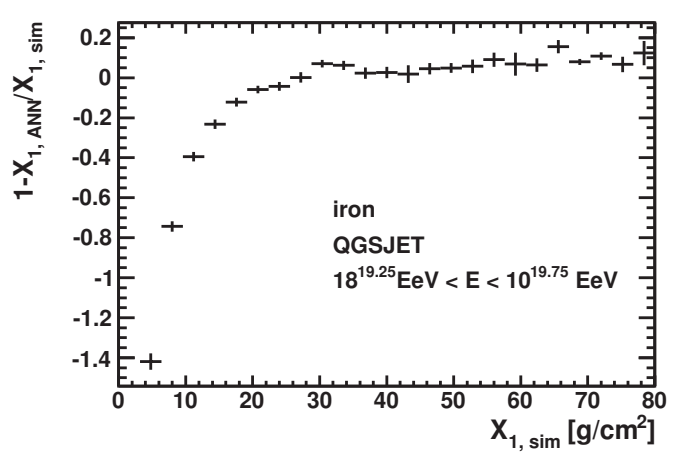

(c)

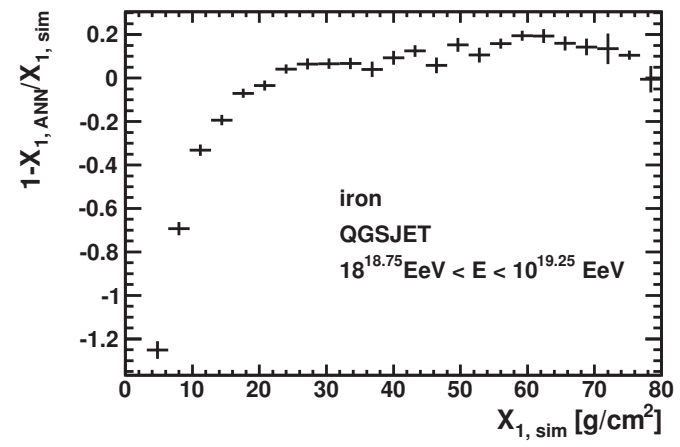

(b)

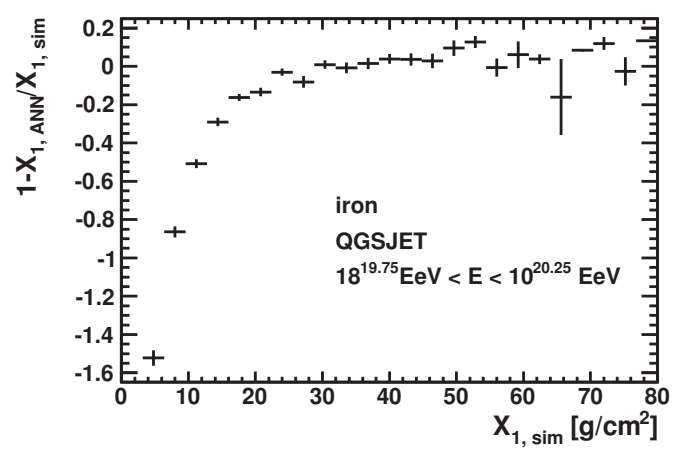

(d)

Figure 6.10: Relative difference between the simulation values and ANN prediction for different energy bins of iron showers. 


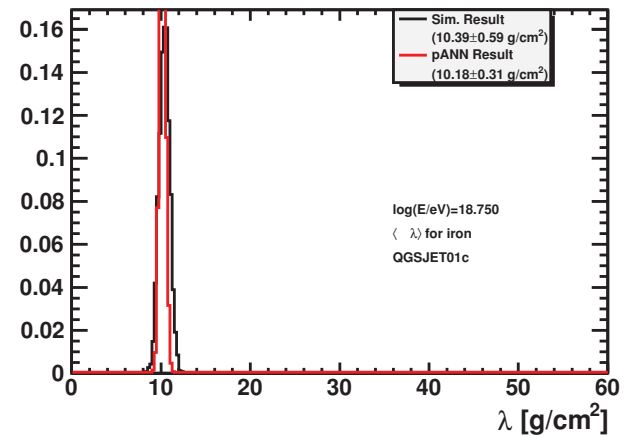

(a)

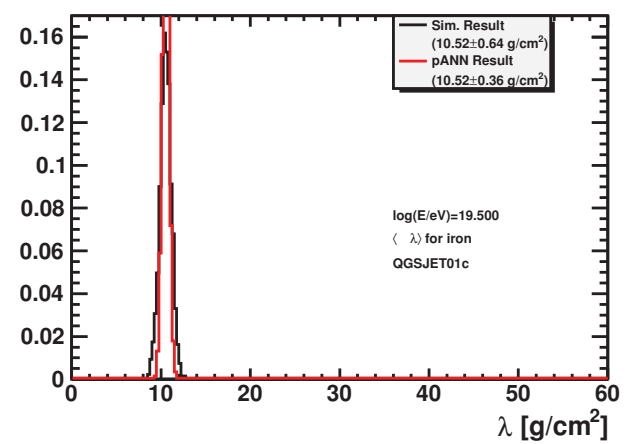

(c)

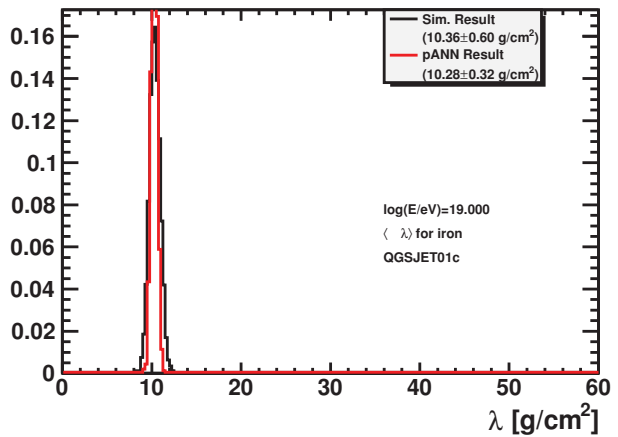

(b)

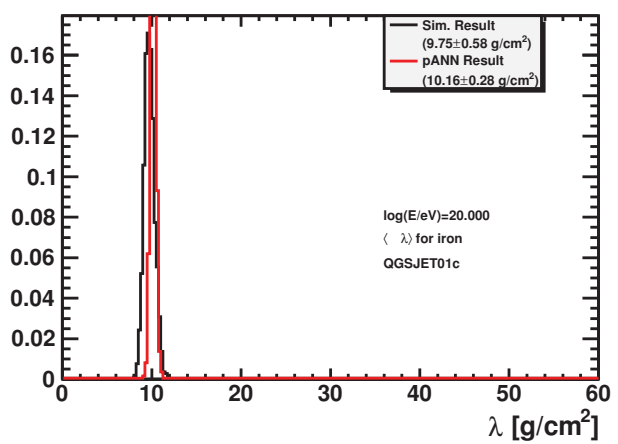

(d)

Figure 6.11: Distribution of mean-free paths constructed by bootstrap method for iron showers generated by QGSJET hadronic interaction model at four different energy bins 


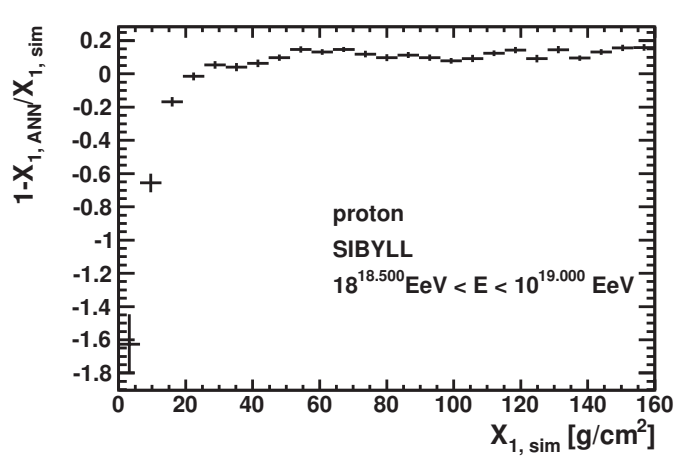

(a)

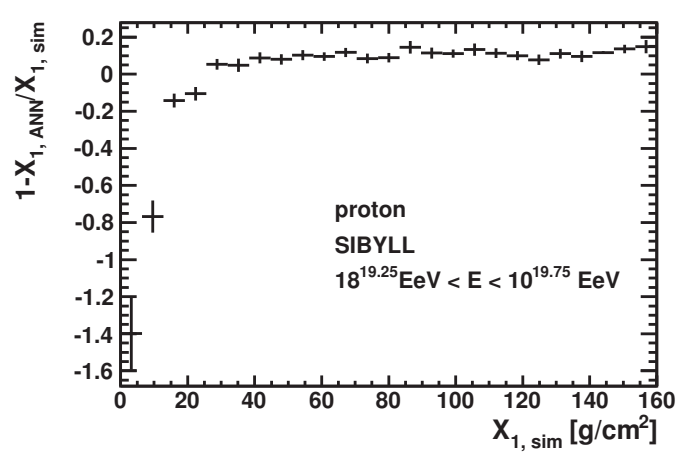

(c)

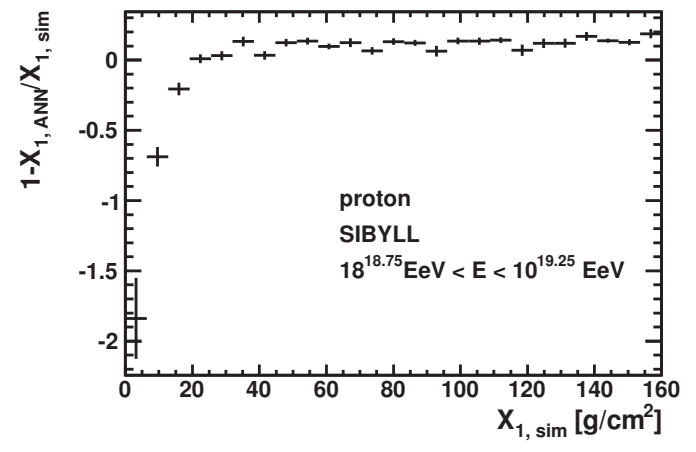

(b)

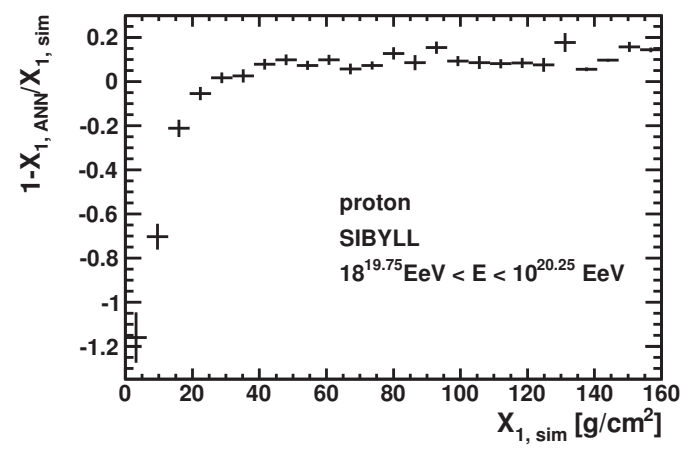

(d)

Figure 6.12: Relative difference between the simulation values and ANN prediction for different energy bins of proton showers. 


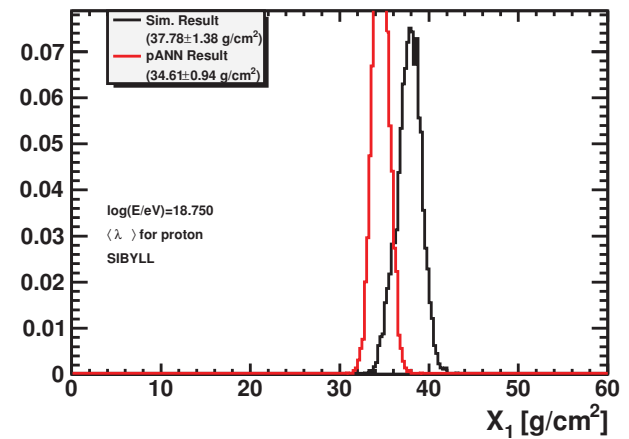

(a)

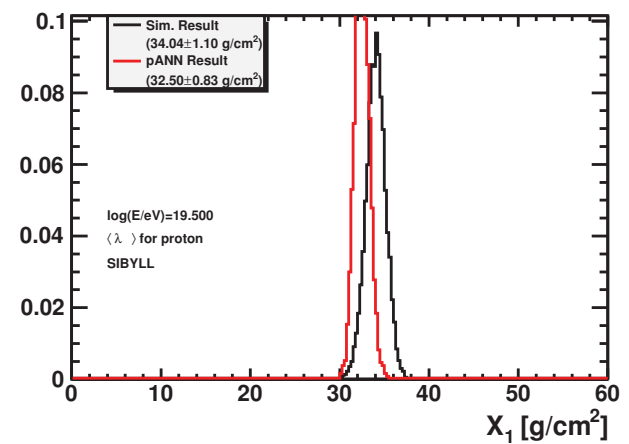

(c)

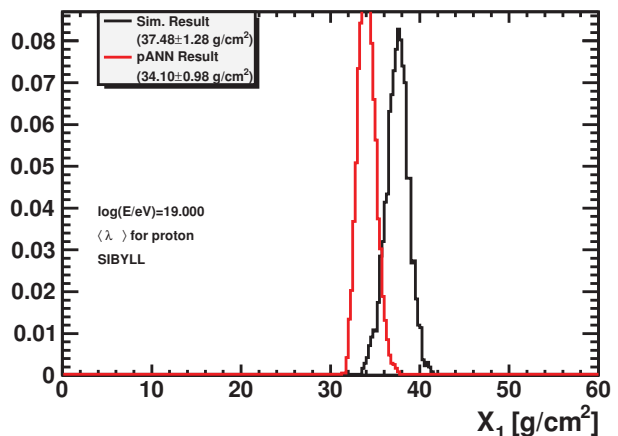

(b)

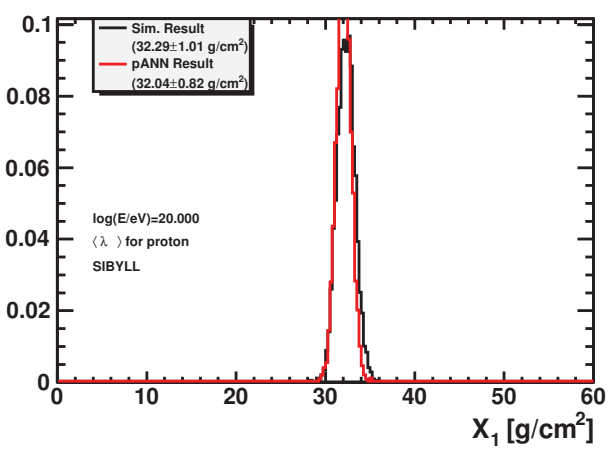

(d)

Figure 6.13: Distribution of mean-free paths constructed by bootstrap method for proton showers generated by SIBYLL hadronic interaction model at four different energy bins 


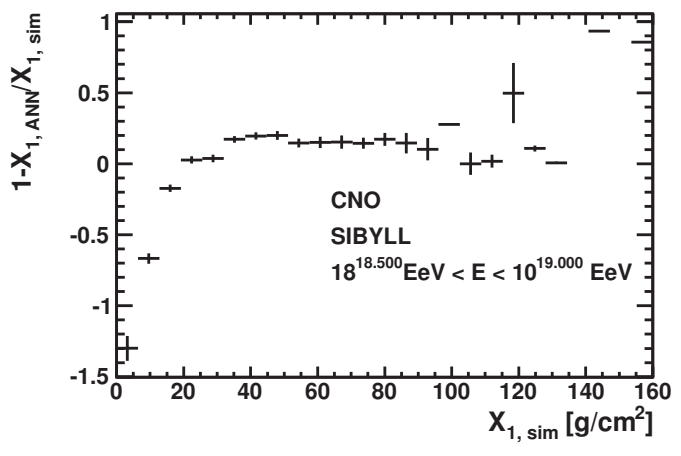

(a)

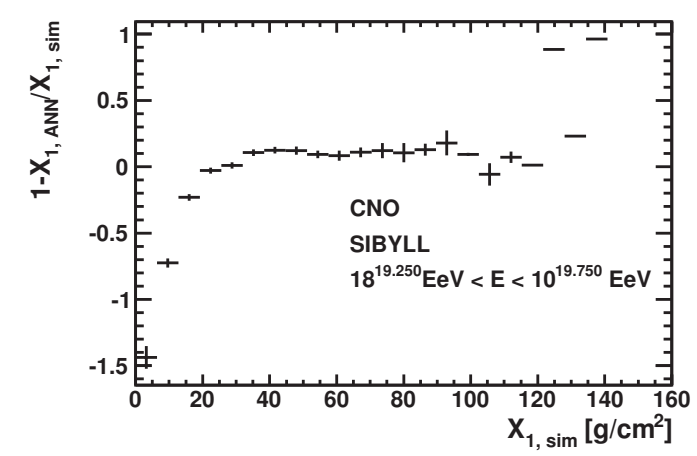

(c)

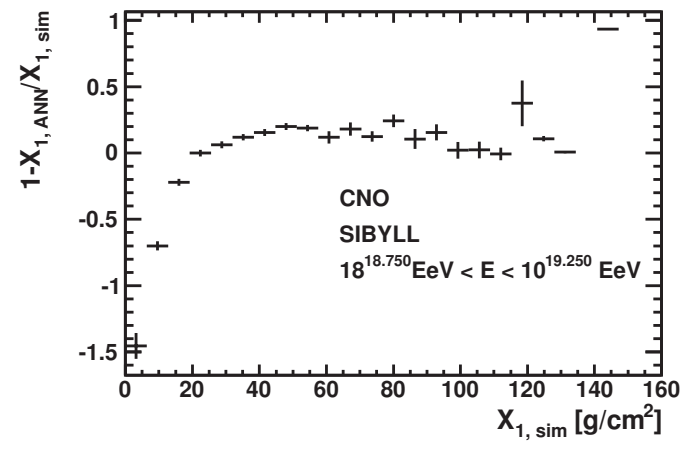

(b)

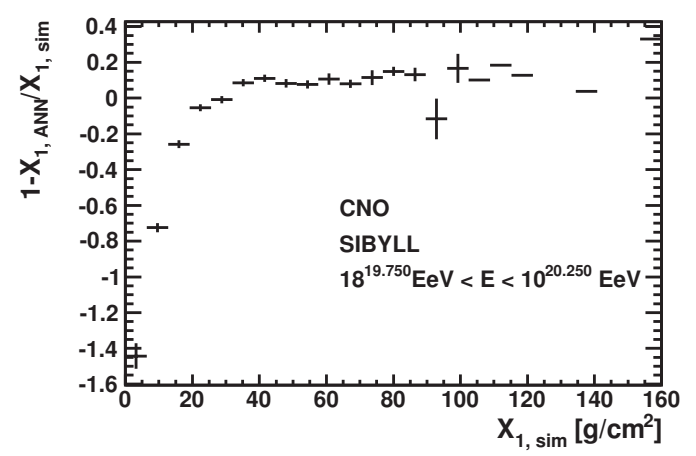

(d)

Figure 6.14: Relative difference between the simulation values and ANN prediction for different energy bins of proton showers. 


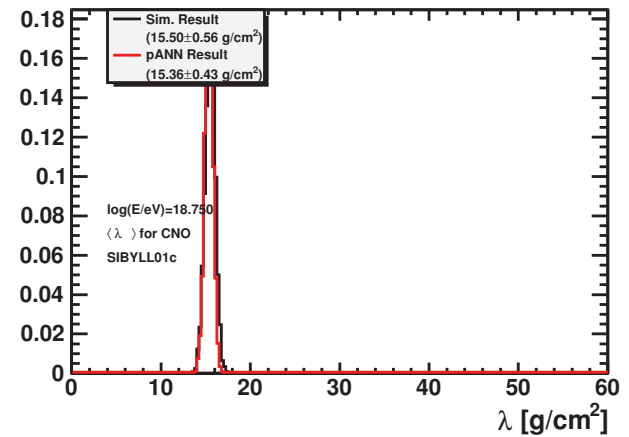

(a)

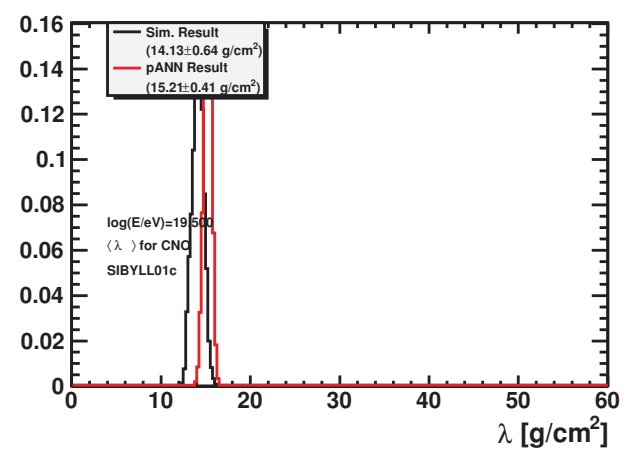

(c)

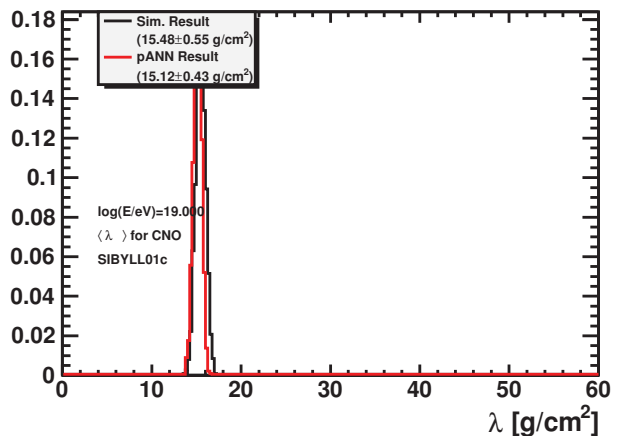

(b)

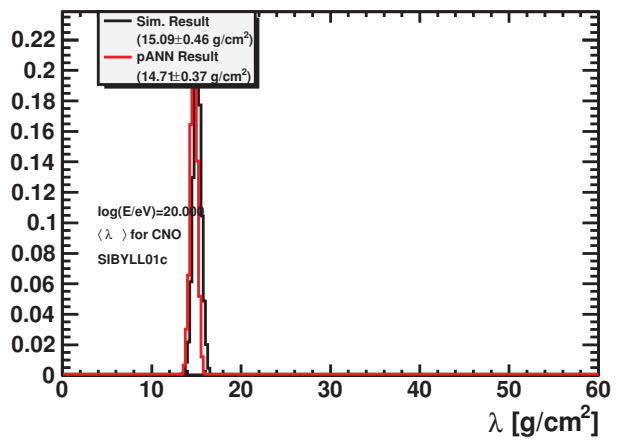

(d)

Figure 6.15: Distribution of mean-free paths constructed by bootstrap method for CNO showers generated by SIBYLL hadronic interaction model at four different energy bins 


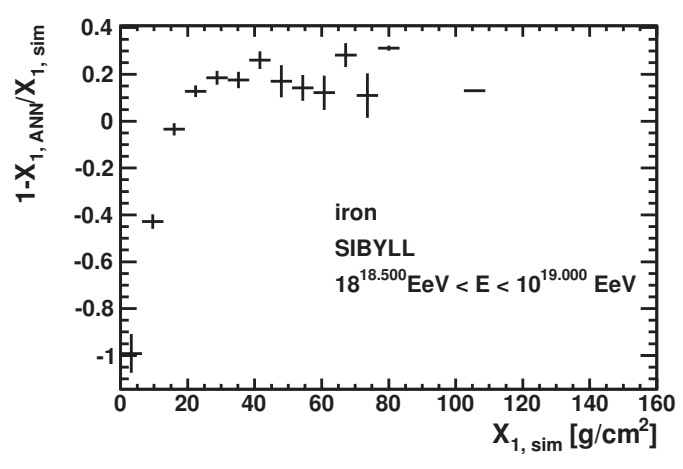

(a)

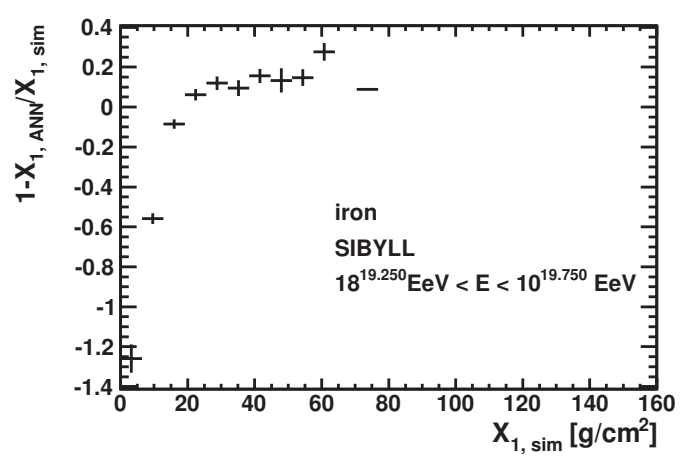

(c)

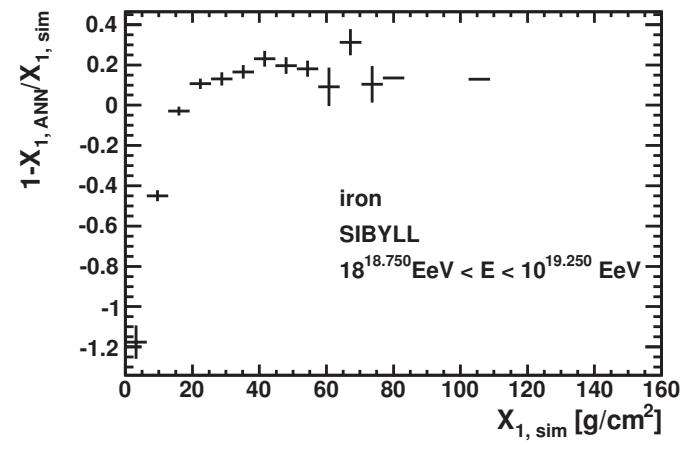

(b)

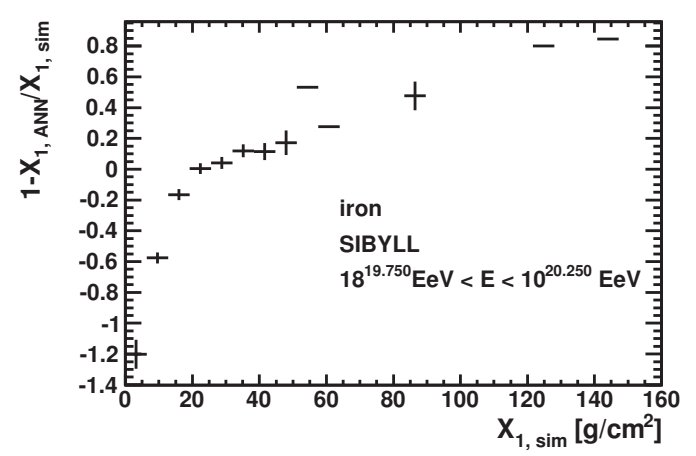

(d)

Figure 6.16: Relative difference between the simulation values and ANN prediction for different energy bins of proton showers. 


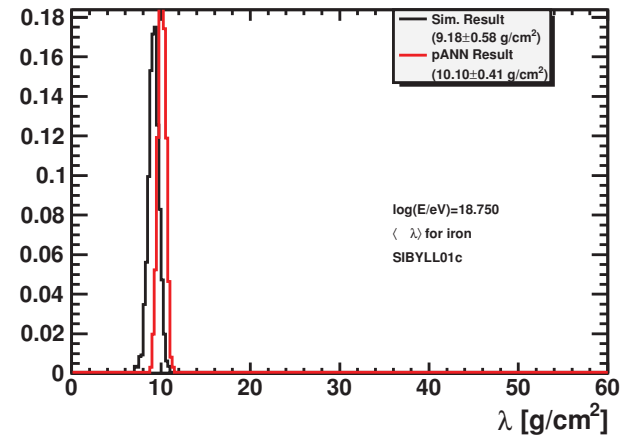

(a)

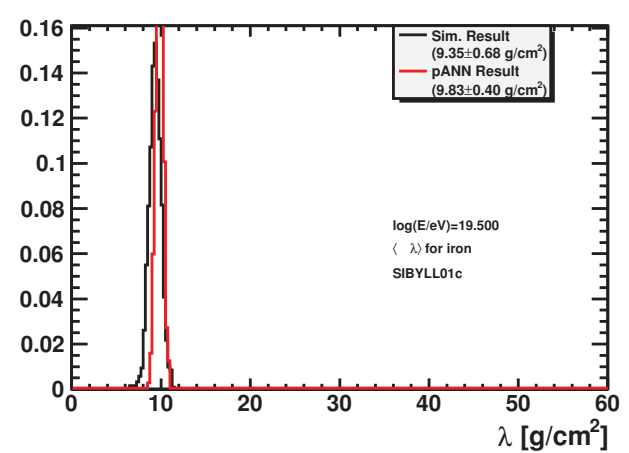

(c)

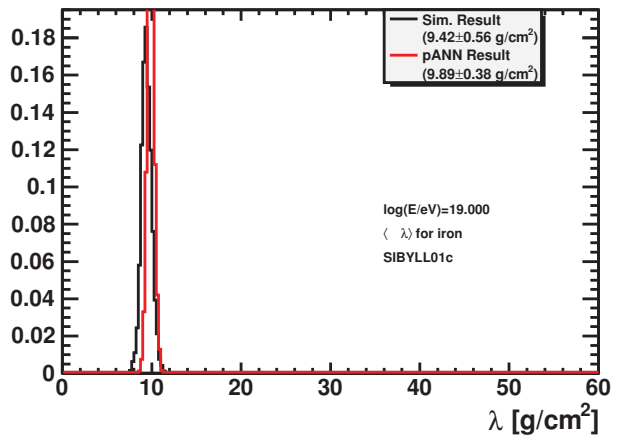

(b)

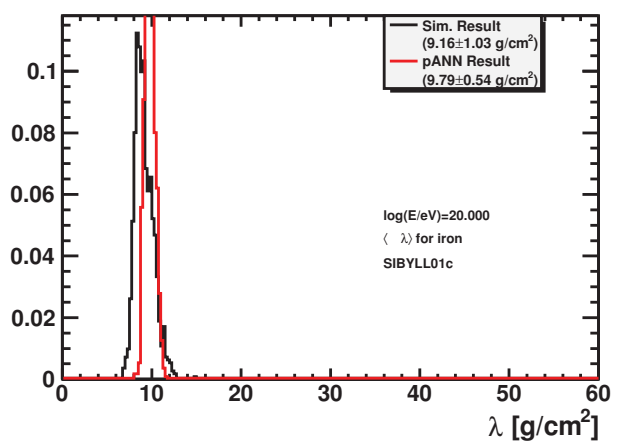

(d)

Figure 6.17: Distribution of mean-free paths constructed by bootstrap method for iron showers generated by SIBYLL hadronic interaction model at four different energy bins 


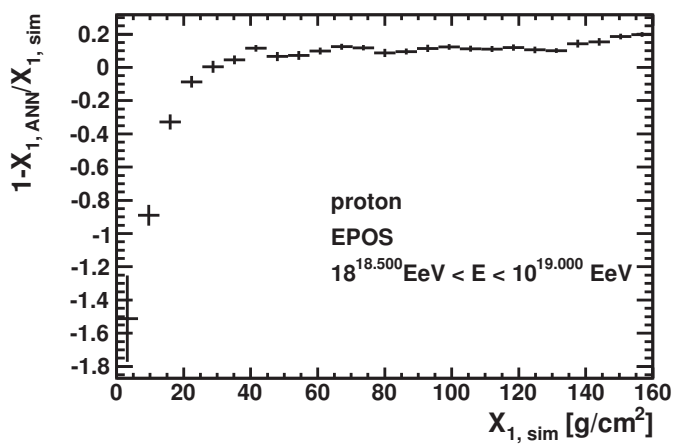

(a)

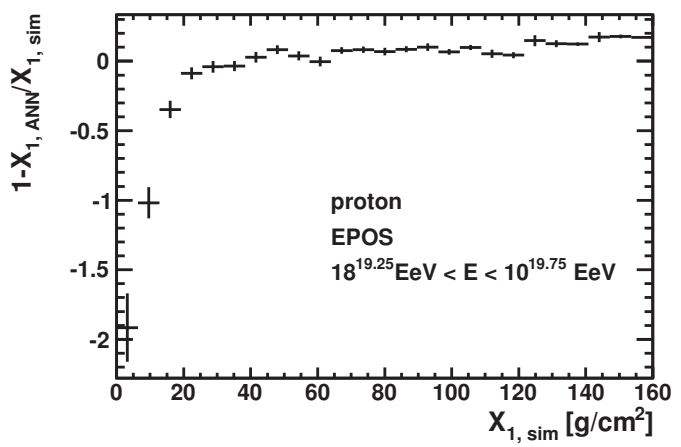

(c)

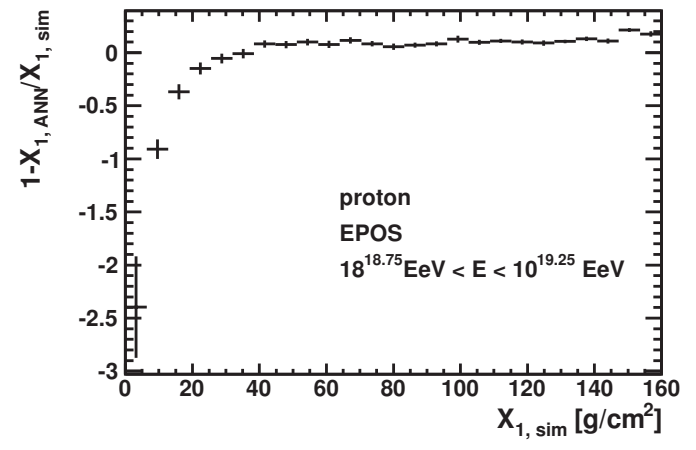

(b)

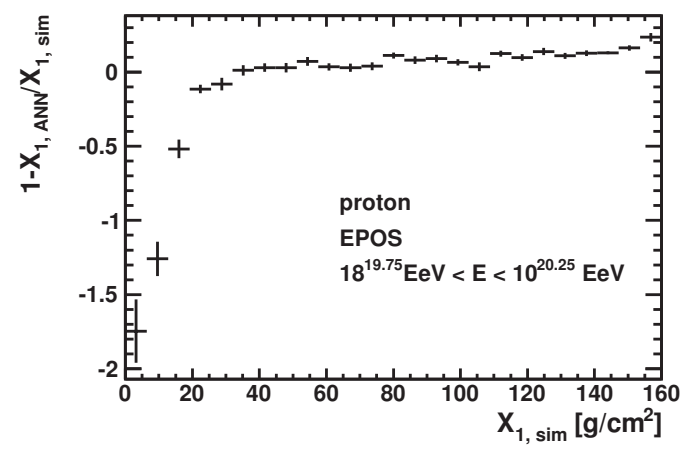

(d)

Figure 6.18: Relative difference between the simulation values and ANN prediction for different energy bins of proton showers. 


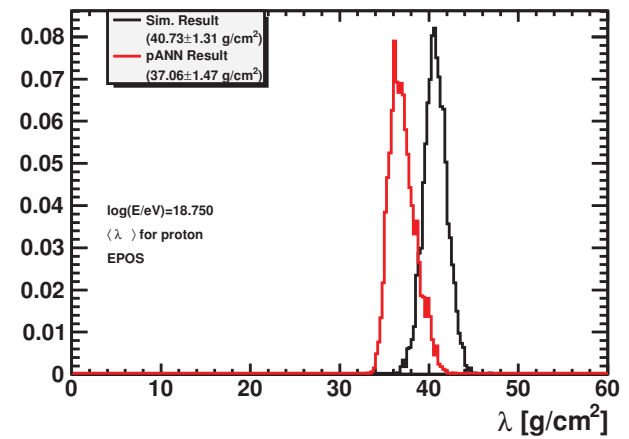

(a)

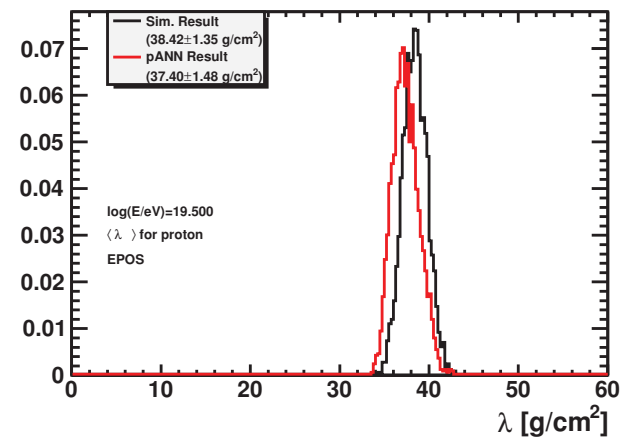

(c)

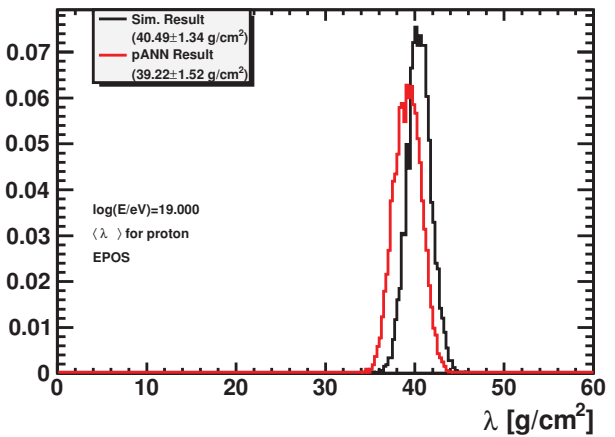

(b)

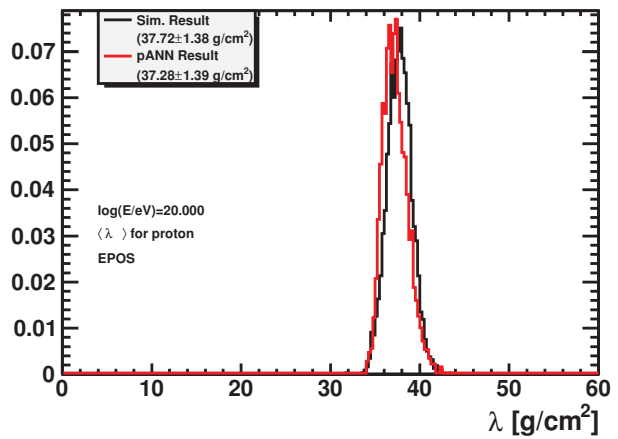

(d)

Figure 6.19: Distribution of mean-free paths constructed by bootstrap method for proton showers generated by EPOS hadronic interaction model at four different energy bins 


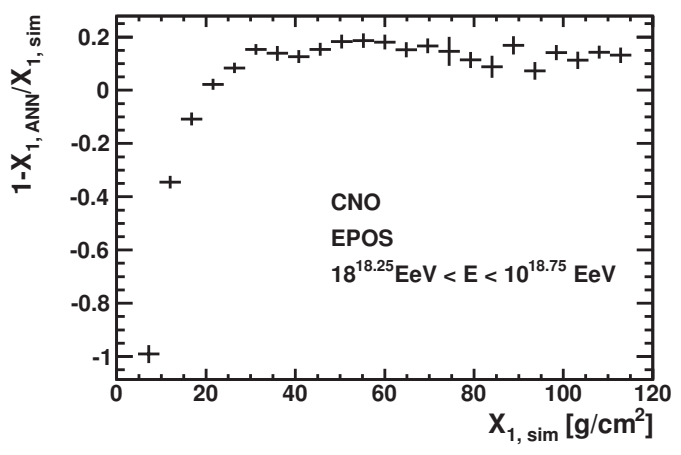

(a)

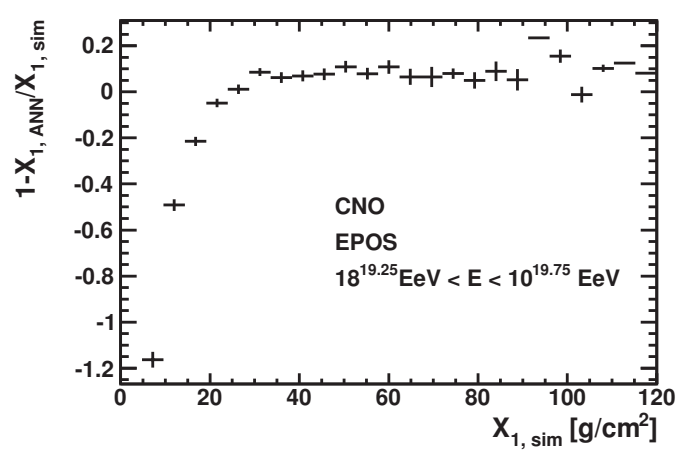

(c)

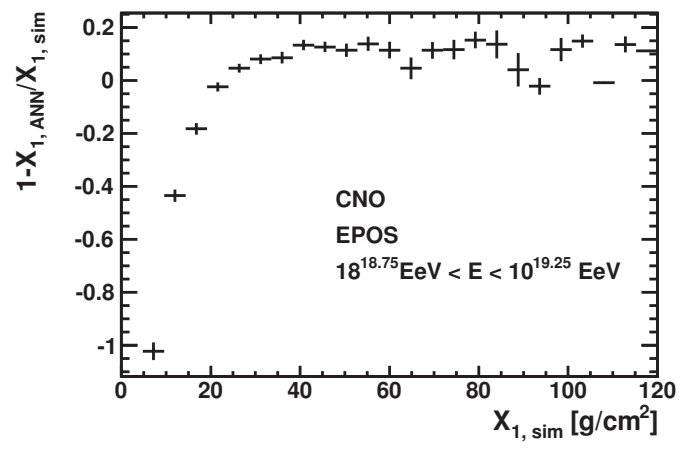

(b)

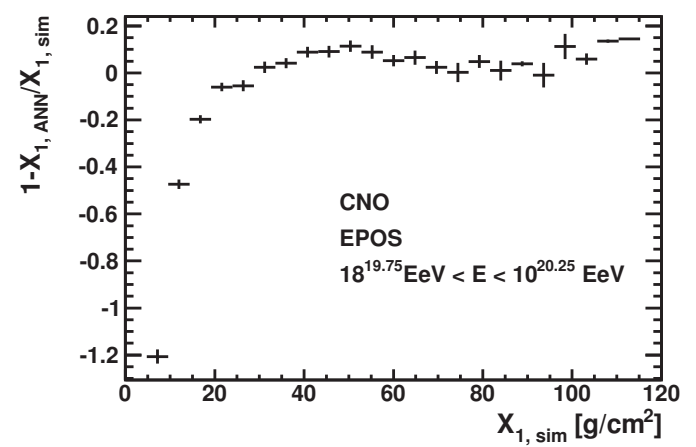

(d)

Figure 6.20: Relative difference between the simulation values and ANN prediction for different energy bins of proton showers. 


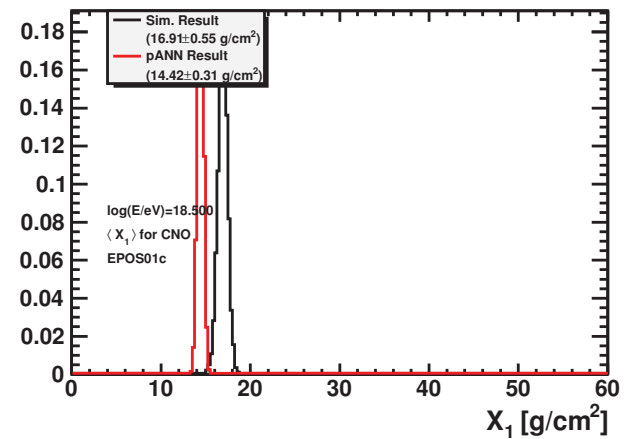

(a)

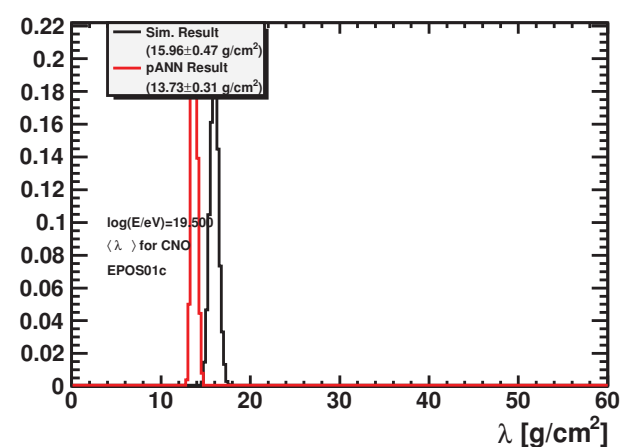

(c)

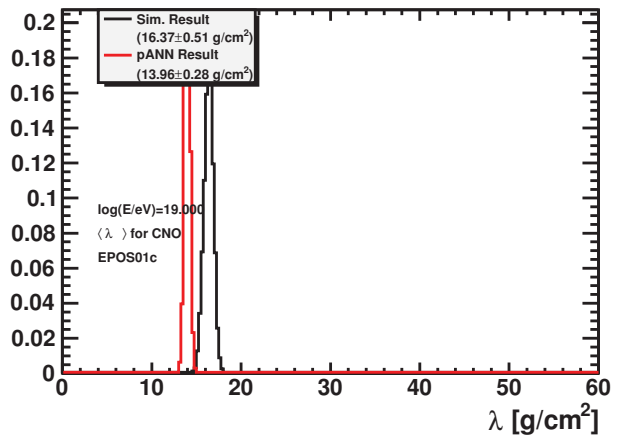

(b)

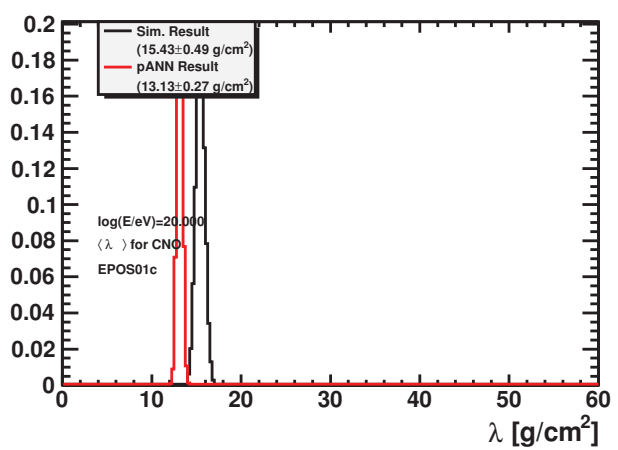

(d)

Figure 6.21: Distribution of mean-free paths constructed by bootstrap method for CNO showers generated by EPOS hadronic interaction model at four different energy bins 


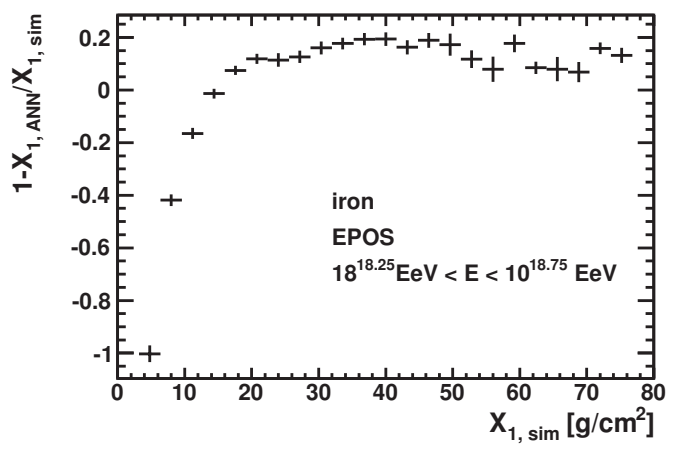

(a)

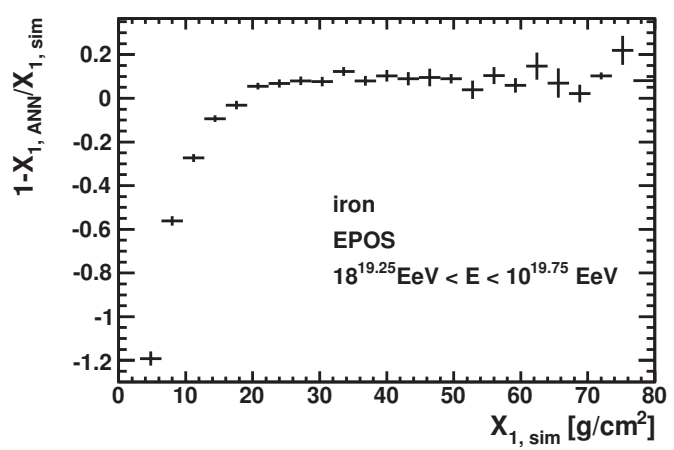

(c)

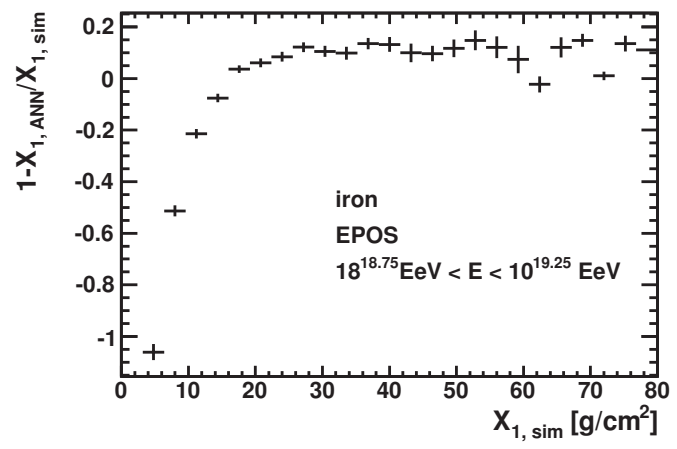

(b)

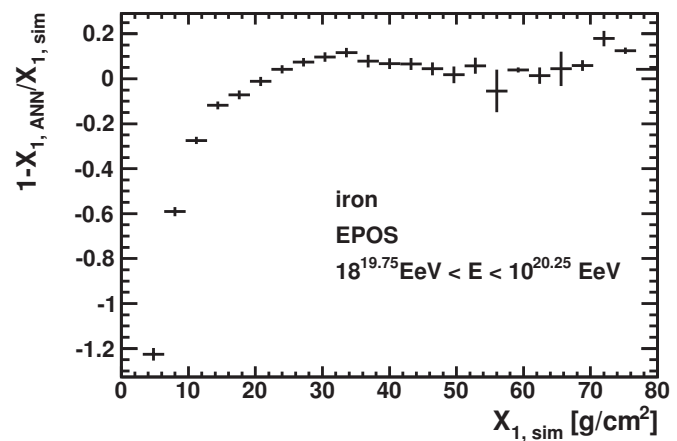

(d)

Figure 6.22: Relative difference between the simulation values and ANN prediction for different energy bins of proton showers. 


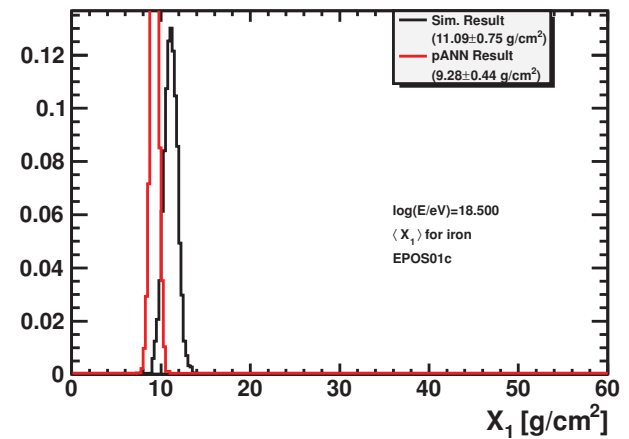

(a)

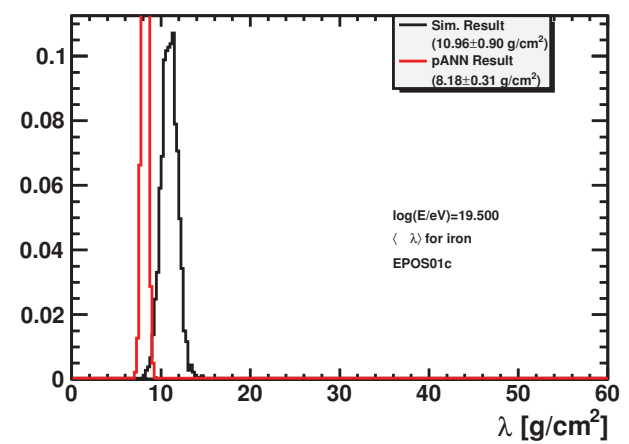

(c)

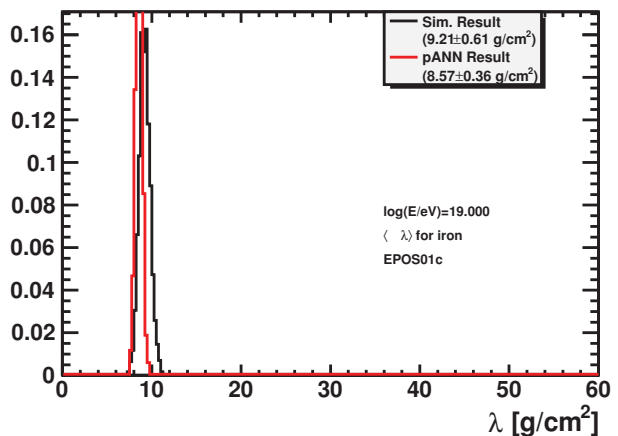

(b)

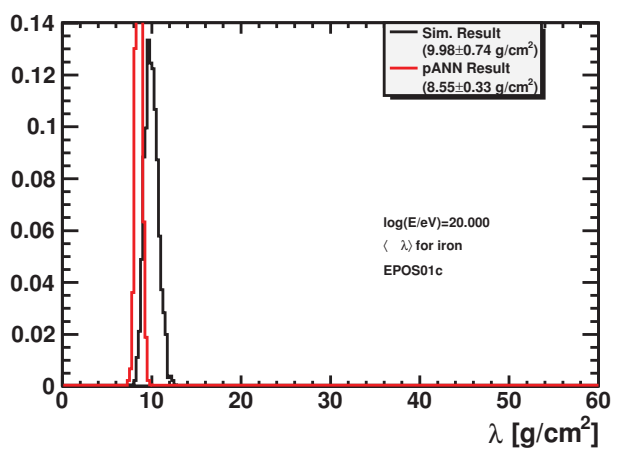

(d)

Figure 6.23: Distribution of mean-free paths constructed by bootstrap method for iron showers generated by EPOS hadronic interaction model at four different energy bins 


\subsubsection{Determining a General Correction Factor}

The mean free paths of the assumed cosmic ray primaries were calculated using the bootstrap method for the hadronic interaction models in four different energy bins (Table 6.3). Furthermore, results of the primaries for the different energy bins are tabulated in Table 6.4. The biases tabulated can be used for correcting the results obtained by pmANN. However, not knowing which the hadronic interaction model was correct prevented us from correcting the results with a single value. Thus, the biases need to be combined to have single value for a given energy bin and primary.

The average value of the biases weighted with their individual uncertainties were computed as shown in Eq. Eqn 6.4.

$$
\bar{x}=\frac{\sum x_{i} w_{i}}{\sum w_{i}}
$$

where $x_{i}$ 's are biases at a given energy bin and a primary particle $w_{i}$ 's are the square of inversed propagated errors in biases, $1 / \sigma_{i}^{2}$ for $\mathrm{i}=\mathrm{QGSJET}$, SIBYLL, EPOS. The weighted averages of the biases were listed in Table 6.5. It can be noted that the correction values for proton and $\mathrm{CNO}$ showers decreased with increasing energy. Unlike proton and $\mathrm{CNO}$ showers, the corrections for iron showers were insignificant.

For the primary particles for four energy bins, corrections were applied and the error were propagated. The corrected values are shown in Table 6.6. Applied corrections have improved the prediction values at all investigated cases except proton showers generated by QGSJET for energies $\log (\mathrm{E} / \mathrm{eV})=18.75$ and 19.50 . Despite the worsening of the results for the mentioned cases, the results are acceptable within the calculated uncertainties. 
Table 6.3: Mean-free paths of cosmic ray primaries for four energy bins. Values were obtained through the distributions of the first interaction lenghts of the generated showers using three different hadronic interaction models.

\begin{tabular}{|c|c|c|c|}
\hline Had.Int. Model & Primary Particle & $\log (\mathrm{E} / \mathrm{eV})$ & Mean-free path $\left[\mathrm{g} / \mathrm{cm}^{2}\right]$ \\
\hline \multirow{12}{*}{ QGSJET } & \multirow{4}{*}{ proton } & 18.75 & $42.46 \pm 1.39$ \\
\hline & & 19.00 & $40.35 \pm 1.26$ \\
\hline & & 19.50 & $41.96 \pm 1.40$ \\
\hline & & 20.00 & $39.60 \pm 1.32$ \\
\hline & \multirow{4}{*}{$\mathrm{CNO}$} & 18.75 & $18.52 \pm 0.52$ \\
\hline & & 19.00 & $18.20 \pm 0.48$ \\
\hline & & 19.50 & $17.47 \pm 0.46$ \\
\hline & & 20.00 & $16.90 \pm 0.46$ \\
\hline & \multirow{4}{*}{ iron } & 18.75 & $10.39 \pm 0.59$ \\
\hline & & 19.00 & $10.36 \pm 0.60$ \\
\hline & & 19.50 & $10.52 \pm 0.64$ \\
\hline & & 20.00 & $9.75 \pm 0.58$ \\
\hline \multirow{12}{*}{ SIBYLL } & \multirow{4}{*}{ proton } & 18.75 & $37.78 \pm 1.38$ \\
\hline & & 19.00 & $37.48 \pm 1.28$ \\
\hline & & 19.50 & $34.04 \pm 1.10$ \\
\hline & & 20.00 & $32.29 \pm 1.01$ \\
\hline & \multirow{4}{*}{$\mathrm{CNO}$} & 18.75 & $15.50 \pm 0.56$ \\
\hline & & 19.00 & $15.48 \pm 0.55$ \\
\hline & & 19.50 & $14.13 \pm 0.64$ \\
\hline & & 20.00 & $15.09 \pm 0.46$ \\
\hline & \multirow{4}{*}{ iron } & 18.75 & $9.18 \pm 0.58$ \\
\hline & & 19.00 & $9.42 \pm 0.56$ \\
\hline & & 19.50 & $9.35 \pm 0.68$ \\
\hline & & 20.00 & $9.16 \pm 1.03$ \\
\hline \multirow{12}{*}{ EPOS } & \multirow{4}{*}{ proton } & 18.75 & $40.73 \pm 1.31$ \\
\hline & & 19.00 & $40.49 \pm 1.34$ \\
\hline & & 19.50 & $38.42 \pm 1.35$ \\
\hline & & 20.00 & $38.42 \pm 1.35$ \\
\hline & \multirow{4}{*}{ CNO } & 18.75 & $16.1 \pm 0.55$ \\
\hline & & 19.00 & $16.37 \pm 0.51$ \\
\hline & & 19.50 & $15.97 \pm 0.47$ \\
\hline & & 20.00 & $15.43 \pm 0.49$ \\
\hline & \multirow{4}{*}{ iron } & 18.75 & $11.09 \pm 0.75$ \\
\hline & & 19.00 & $9.21 \pm 0.61$ \\
\hline & & 19.50 & $10.96 \pm 0.90$ \\
\hline & & 20.00 & $9.98 \pm 0.74$ \\
\hline
\end{tabular}


Table 6.4: Expected value of mean-free path of different primaries for shower simulated with three hadronic interaction models at four energy bins. Simulation values, pmANN values and biases were in $\mathrm{g} / \mathrm{cm}^{2}$.

\begin{tabular}{|c|c|c|c|c|c|c|}
\hline $\begin{array}{c}\text { Hadronic } \\
\text { Model }\end{array}$ & primary & $\log (\mathrm{E} / \mathrm{eV})$ & $\begin{array}{c}\text { Number of } \\
\text { Showers }\end{array}$ & $\begin{array}{l}\text { Sim. Value } \\
{\left[\mathrm{g} / \mathrm{cm}^{2}\right]}\end{array}$ & $\begin{array}{c}\text { pmANN Value } \\
{\left[\mathrm{g} / \mathrm{cm}^{2}\right]}\end{array}$ & $\begin{array}{c}\text { Bias } \\
{\left[\mathrm{g} / \mathrm{cm}^{2}\right]}\end{array}$ \\
\hline \multirow{12}{*}{ QGSJET } & \multirow{4}{*}{ Proton } & 18.75 & 3634 & $42.46 \pm 1.39$ & $43.25 \pm 2.70$ & $-0.79 \pm 3.04$ \\
\hline & & 19.00 & 3654 & $40.35 \pm 1.26$ & $40.75 \pm 2.02$ & $-0.40 \pm 2.38$ \\
\hline & & 19.50 & 3679 & $41.96 \pm 1.40$ & $43.87 \pm 2.33$ & $-1.91 \pm 2.72$ \\
\hline & & 20.00 & 3443 & $39.60 \pm 1.32$ & $41.28 \pm 2.51$ & $-1.67 \pm 2.84$ \\
\hline & \multirow{4}{*}{$\mathrm{CNO}$} & 18.75 & 5222 & $18.52 \pm 0.52$ & $16.61 \pm 0.34$ & $1.91 \pm 0.62$ \\
\hline & & 19.00 & 5314 & $18.20 \pm 0.48$ & $17.13 \pm 0.35$ & $1.07 \pm 0.59$ \\
\hline & & 19.50 & 5313 & $17.47 \pm 0.46$ & $16.47 \pm 0.33$ & $1.00 \pm 0.57$ \\
\hline & & 20.00 & 5190 & $16.90 \pm 0.46$ & $16.48 \pm 0.32$ & $0.42 \pm 0.56$ \\
\hline & \multirow{4}{*}{ Iron } & 18.75 & 2570 & $10.39 \pm 0.59$ & $10.18 \pm 0.31$ & $0.21 \pm 0.67$ \\
\hline & & 19.00 & 2530 & $10.36 \pm 0.60$ & $10.28 \pm 0.32$ & $0.08 \pm 0.68$ \\
\hline & & 19.50 & 2576 & $10.52 \pm 0.64$ & $10.52 \pm 0.36$ & $-0.00 \pm 0.73$ \\
\hline & & 20.00 & 2468 & $9.75 \pm 0.58$ & $10.16 \pm 0.28$ & $-0.41 \pm 0.65$ \\
\hline \multirow{12}{*}{ SIBYLL } & \multirow{4}{*}{ Proton } & 18.75 & 3541 & $37.78 \pm 1.38$ & $34.61 \pm 0.94$ & $3.17 \pm 1.67$ \\
\hline & & 19.00 & 3501 & $37.48 \pm 1.28$ & $34.10 \pm 0.98$ & $3.38 \pm 1.61$ \\
\hline & & 19.50 & 3640 & $34.04 \pm 1.10$ & $32.50 \pm 0.83$ & $1.54 \pm 1.37$ \\
\hline & & 20.00 & 3460 & $32.29 \pm 1.01$ & $32.04 \pm 0.82$ & $0.25 \pm 1.30$ \\
\hline & \multirow{4}{*}{$\mathrm{CNO}$} & 18.75 & 3723 & $15.50 \pm 0.56$ & $15.36 \pm 0.43$ & $0.14 \pm 0.71$ \\
\hline & & 19.00 & 3648 & $15.48 \pm 0.55$ & $15.12 \pm 0.43$ & $0.36 \pm 0.70$ \\
\hline & & 19.50 & 3620 & $14.13 \pm 0.64$ & $15.21 \pm 0.41$ & $-1.08 \pm 0.76$ \\
\hline & & 20.00 & 4982 & $15.09 \pm 0.46$ & $14.71 \pm 0.37$ & $0.38 \pm 0.58$ \\
\hline & \multirow{4}{*}{ Iron } & 18.75 & 2582 & $9.18 \pm 0.58$ & $10.10 \pm 0.41$ & $-0.92 \pm 0.72$ \\
\hline & & 19.00 & 2546 & $9.42 \pm 0.56$ & $9.89 \pm 0.38$ & $-0.47 \pm 0.68$ \\
\hline & & 19.50 & 2453 & $9.35 \pm 0.68$ & $9.83 \pm 0.40$ & $-0.48 \pm 0.79$ \\
\hline & & 20.00 & 1112 & $9.16 \pm 1.03$ & $9.79 \pm 0.54$ & $-0.63 \pm 1.16$ \\
\hline \multirow{12}{*}{ EPOS } & \multirow{4}{*}{ Proton } & 18.75 & 3277 & $40.73 \pm 1.31$ & $37.06 \pm 1.47$ & $3.67 \pm 1.96$ \\
\hline & & 19.00 & 3247 & $40.49 \pm 1.34$ & $39.22 \pm 1.52$ & $1.28 \pm 2.02$ \\
\hline & & 19.50 & 3260 & $38.42 \pm 1.35$ & $37.40 \pm 1.48$ & $1.02 \pm 2.01$ \\
\hline & & 20.00 & 3182 & $37.72 \pm 1.38$ & $37.28 \pm 1.39$ & $0.44 \pm 1.96$ \\
\hline & \multirow{4}{*}{$\mathrm{CNO}$} & 18.75 & 5036 & $16.94 \pm 0.55$ & $14.16 \pm 0.32$ & $2.77 \pm 0.64$ \\
\hline & & 19.00 & 5066 & $16.37 \pm 0.51$ & $13.96 \pm 0.28$ & $2.41 \pm 0.58$ \\
\hline & & 19.50 & 5038 & $15.96 \pm 0.47$ & $13.73 \pm 0.31$ & $2.22 \pm 0.56$ \\
\hline & & 20.00 & 5009 & $15.43 \pm 0.49$ & $13.13 \pm 0.27$ & $2.30 \pm 0.56$ \\
\hline & \multirow{4}{*}{ Iron } & 18.75 & 1902 & $10.05 \pm 0.64$ & $8.96 \pm 0.41$ & $1.09 \pm 0.76$ \\
\hline & & 19.00 & 1862 & $9.21 \pm 0.61$ & $8.57 \pm 0.36$ & $0.64 \pm 0.71$ \\
\hline & & 19.50 & 1810 & $10.96 \pm 0.90$ & $8.18 \pm 0.31$ & $2.78 \pm 0.95$ \\
\hline & & 20.00 & 1798 & $9.98 \pm 0.74$ & $8.55 \pm 0.33$ & $1.43 \pm 0.81$ \\
\hline
\end{tabular}


Table 6.5: Weighted average of biases of different primaries for shower simulated with three hadronic interaction models at four energy bins

\begin{tabular}{|c|c|c|}
\hline primary & $\log (\mathrm{E} / \mathrm{eV})$ & Weighted averaged bias $\left[\mathrm{g} / \mathrm{cm}^{2}\right]$ \\
\hline \multirow{4}{*}{ Proton } & 18.75 & $2.78 \pm 1.18$ \\
\cline { 2 - 3 } & 19.00 & $1.92 \pm 1.11$ \\
\cline { 2 - 3 } & 19.50 & $0.75 \pm 1.05$ \\
\cline { 2 - 3 } CNO & 20.00 & $0.06 \pm 1.03$ \\
\hline \multirow{4}{*}{ Iron } & 18.75 & $1.71 \pm 0.38$ \\
\cline { 2 - 3 } & 19.00 & $1.39 \pm 0.36$ \\
\cline { 2 - 3 } & 19.50 & $1.04 \pm 0.35$ \\
\cline { 2 - 3 } & 20.00 & $1.05 \pm 0.33$ \\
\hline \multirow{4}{*}{} & 18.75 & $0.10 \pm 0.41$ \\
\cline { 2 - 3 } & 19.00 & $0.07 \pm 0.40$ \\
\cline { 2 - 3 } & 19.50 & $0.50 \pm 0.47$ \\
\cline { 2 - 3 } & 20.00 & $0.16 \pm 0.47$ \\
\hline
\end{tabular}


Table 6.6: Corrected expected values of mean-free paths of different primaries for shower simulated with three hadronic interaction models at four energy bins. Simulation values and pmANN values were in $\mathrm{g} / \mathrm{cm}^{2}$.

\begin{tabular}{|c|c|c|c|c|c|}
\hline Had. Model & primary & $\log (\mathrm{E} / \mathrm{eV})$ & $\begin{array}{l}\text { Number } \\
\text { of showers }\end{array}$ & $\begin{array}{l}\text { Sim. Value } \\
{\left[\mathrm{g} / \mathrm{cm}^{2}\right]}\end{array}$ & $\begin{array}{c}\text { pmANN Value } \\
{\left[\mathrm{g} / \mathrm{cm}^{2}\right]}\end{array}$ \\
\hline \multirow{12}{*}{ QGSJET } & \multirow{4}{*}{ Proton } & 18.75 & 3634 & $42.46 \pm 1.39$ & $46.03 \pm 2.95$ \\
\hline & & 19.00 & 3654 & $40.35 \pm 1.26$ & $42.67 \pm 2.30$ \\
\hline & & 19.50 & 3679 & $41.96 \pm 1.40$ & $44.62 \pm 2.56$ \\
\hline & & 20.00 & 3443 & $39.60 \pm 1.32$ & $41.34 \pm 2.71$ \\
\hline & \multirow{4}{*}{$\mathrm{CNO}$} & 18.75 & 5222 & $18.52 \pm 0.52$ & $18.32 \pm 0.51$ \\
\hline & & 19.00 & 5314 & $18.20 \pm 0.48$ & $18.52 \pm 0.50$ \\
\hline & & 19.50 & 5313 & $17.47 \pm 0.46$ & $17.51 \pm 0.48$ \\
\hline & & 20.00 & 5190 & $16.90 \pm 0.46$ & $17.53 \pm 0.46$ \\
\hline & \multirow{4}{*}{ Iron } & 18.75 & 2570 & $10.39 \pm 0.59$ & $10.28 \pm 0.51$ \\
\hline & & 19.00 & 2530 & $10.36 \pm 0.60$ & $10.35 \pm 0.51$ \\
\hline & & 19.50 & 2576 & $10.52 \pm 0.64$ & $11.02 \pm 0.59$ \\
\hline & & 20.00 & 2468 & $9.75 \pm 0.58$ & $10.32 \pm 0.55$ \\
\hline \multirow{12}{*}{ SIBYLL } & \multirow{4}{*}{ Proton } & 18.75 & 3541 & $37.78 \pm 1.38$ & $37.39 \pm 1.51$ \\
\hline & & 19.00 & 3501 & $37.48 \pm 1.28$ & $36.02 \pm 1.48$ \\
\hline & & 19.50 & 3640 & $34.04 \pm 1.10$ & $33.25 \pm 1.34$ \\
\hline & & 20.00 & 3460 & $32.29 \pm 1.01$ & $32.10 \pm 1.32$ \\
\hline & \multirow{4}{*}{$\mathrm{CNO}$} & 18.75 & 3723 & $15.50 \pm 0.56$ & $17.07 \pm 0.57$ \\
\hline & & 19.00 & 3648 & $15.48 \pm 0.55$ & $16.51 \pm 0.56$ \\
\hline & & 19.50 & 3620 & $14.13 \pm 0.64$ & $16.25 \pm 0.54$ \\
\hline & & 20.00 & 4982 & $15.09 \pm 0.46$ & $15.76 \pm 0.50$ \\
\hline & \multirow{4}{*}{ Iron } & 18.75 & 2582 & $9.18 \pm 0.58$ & $10.20 \pm 0.58$ \\
\hline & & 19.00 & 2546 & $9.42 \pm 0.56$ & $9.96 \pm 0.55$ \\
\hline & & 19.50 & 2453 & $9.35 \pm 0.68$ & $10.33 \pm 0.62$ \\
\hline & & 20.00 & 1112 & $9.16 \pm 1.03$ & $9.95 \pm 0.72$ \\
\hline \multirow{12}{*}{ EPOS } & \multirow{4}{*}{ Proton } & 18.75 & 3277 & $40.73 \pm 1.31$ & $39.84 \pm 1.89$ \\
\hline & & 19.00 & 3247 & $40.49 \pm 1.34$ & $41.14 \pm 1.88$ \\
\hline & & 19.50 & 3260 & $38.42 \pm 1.35$ & $38.15 \pm 1.81$ \\
\hline & & 20.00 & 3182 & $37.72 \pm 1.38$ & $37.34 \pm 1.73$ \\
\hline & \multirow{4}{*}{$\mathrm{CNO}$} & 18.75 & 5036 & $16.94 \pm 0.55$ & $15.87 \pm 0.50$ \\
\hline & & 19.00 & 5066 & $16.37 \pm 0.51$ & $15.35 \pm 0.46$ \\
\hline & & 19.50 & 5038 & $15.96 \pm 0.47$ & $14.77 \pm 0.47$ \\
\hline & & 20.00 & 5009 & $15.43 \pm 0.49$ & $14.18 \pm 0.43$ \\
\hline & \multirow{4}{*}{ Iron } & 18.75 & 1902 & $10.05 \pm 0.64$ & $9.06 \pm 0.58$ \\
\hline & & 19.00 & 1862 & $9.21 \pm 0.61$ & $8.64 \pm 0.54$ \\
\hline & & 19.50 & 1810 & $10.96 \pm 0.90$ & $8.68 \pm 0.56$ \\
\hline & & 20.00 & 1798 & $9.98 \pm 0.74$ & $8.71 \pm 0.57$ \\
\hline
\end{tabular}




\section{Chapter 7 Mixed Cosmic Ray Scenario and Composition Analysis}

For observed events, since the mixture ratios have not been found yet, it is not practical to attempt to determine the first interaction lengths of the cosmic rays. Suitable chosen break points for the first interaction length distribution can be employed to differentiate the mixture and compute the mean-free paths of individual components of the mixture. In Figure 7.1, a two-component composition was shown with arbitrary primaries. Two distributions were superposed and as a consequence the break point will partition the distribution into two parts. The part after the break point, or the tail of distribution, will carry the information about the light particle whereas the part before the break point, the head of distribution, will have information about both particles. Using the information from the tail will dis-entangle the heavy particle information in the head of distribution.

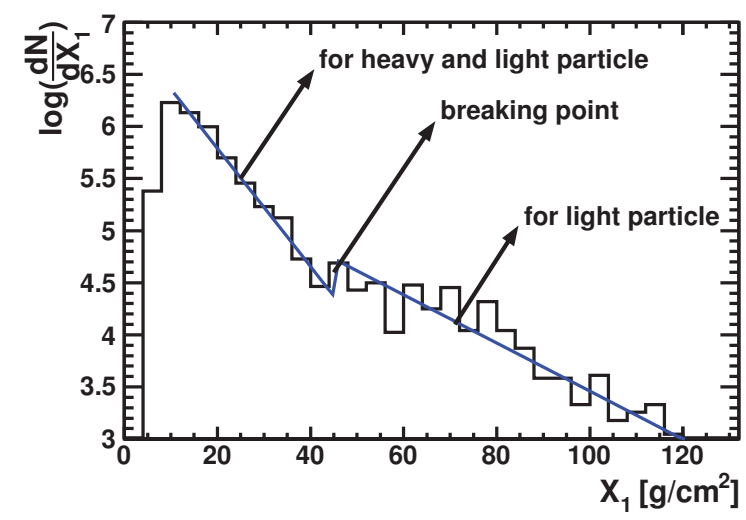

Figure 7.1: A sample distribution of $X_{1}$ values for a mixed composition in semi-log scale.

A simplistic approach employing an analytical solution can be developed. Even though, different values for the break point can be calculated for each hadronic interaction model, a generic break point should be computed that will work for the hadronic interaction mod- 
els.

For the reasons mentioned in 2.4, we carried out the work for proton, CNO and iron mixtures. Sensitivity analyses were also run for virtual primaries with arbitrary meanfree paths. Average values for mean-free paths for the mentioned primary particles were computed by the bootstrap method. Randomly chosen first interaction lengths from the showers generated by three hadronic interaction models constructed the vector, $\mathbf{X}$ (see section 6.2.2.1). Expected values for the mean-free paths were calculated by the bootstrap method. The calculated values for the expected mean-free paths of primaries are 43.75 $\mathrm{g} / \mathrm{cm}^{2}, 17.72 \mathrm{~g} / \mathrm{cm}^{2}$ and $10.02 \mathrm{~g} / \mathrm{cm}^{2}$ for proton, CNO and iron, respectively.

\subsection{Finding the break point for a two-component composition}

For a given primary, the $\mathrm{X}_{1}$ distribution follows an exponential distribution which can be characterized with a exponential function:

$$
\begin{aligned}
P_{1}\left(\mathrm{X}_{1}\right) & =\frac{d N}{d \mathrm{X}_{1}} \\
& =\kappa_{1} \exp \left(-\lambda_{1} \times \mathrm{X}_{1}\right)
\end{aligned}
$$

where $\kappa$ is a normalization factor. The total number of events can be calculated as:

$$
\begin{aligned}
N_{1, \text { total }} & =\int_{0}^{\infty} P_{1}\left(\mathrm{X}_{1}\right) d \mathrm{X}_{1} \\
N_{1, \text { total }} & =\frac{\kappa_{1}}{\lambda_{1}}
\end{aligned}
$$

Then, if one assumes a mixed composition of two primaries, the distribution can be represented as superposition of two individual exponential distributions:

$$
\begin{aligned}
P_{\text {total }}\left(\mathrm{X}_{1}\right) & =P_{1}\left(\mathrm{X}_{1}\right)+P_{2}\left(\mathrm{X}_{1}\right) \\
& =\kappa_{1} \exp \left(-\lambda_{1} \times \mathrm{X}_{1}\right)+\kappa_{2} \exp \left(-\lambda_{2} \times \mathrm{X}_{1}\right)
\end{aligned}
$$

Similarly, total number of events is:

$$
N_{\text {total }}=\int_{0}^{\infty}\left(P_{1}\left(\mathrm{X}_{1}\right)+P_{2}\left(\mathrm{X}_{1}\right)\right) d \mathrm{X}_{1}=\frac{\kappa_{1}}{\lambda_{1}}+\frac{\kappa_{2}}{\lambda_{2}}
$$


The solution of the Eqn.Eqn 7.3 is complicated and in most cases impossible to solve analytically for a given distribution. Thus, to remove the complication on the equation, the given distribution can be segmented into two or more regions in which a single composition is dominant. Then, the solution simplifies to fitting a single exponential distribution rather than super-imposed two exponential distributions. The region in which one can assume a singular composition can be found analytically. Let the region be bounded by $[\alpha, \infty]$ where $\alpha$ will be called as "break point" and this region is occupied with a specific fraction $(\beta)$ of the lighter particle distribution $P_{1}$ and a specific fraction $(1-\beta)$ of the heavier fraction of the heavier distribution $P_{2}$. Then, total number of events in the bounded region is:

$$
\begin{aligned}
N_{\text {total }, \alpha} & =\int_{\alpha}^{\infty}\left(P_{1}\left(\mathrm{X}_{1}\right)+P_{2}\left(\mathrm{X}_{1}\right)\right) d \mathrm{X}_{1} \\
& =\frac{\kappa_{1}}{\lambda_{1}} \exp \left(-\lambda_{1} \alpha\right)+\frac{\kappa_{2}}{\lambda_{2}} \exp \left(-\lambda_{2} \alpha\right) \\
& =N_{1, \alpha}+N_{2, \alpha}
\end{aligned}
$$

Then,

$$
\begin{aligned}
\frac{\beta}{1-\beta} & =\frac{N_{1, \alpha}}{N_{2, \alpha}} \\
& =\frac{\frac{\kappa_{1}}{\lambda_{1}} \exp \left(-\lambda_{1} \alpha\right)}{\frac{\kappa_{2}}{\lambda_{2}} \exp \left(-\lambda_{2} \alpha\right)} \\
& =\left(\frac{N_{1, \text { total }}}{N_{2, \text { total }}}\right) \frac{\exp \left(-\lambda_{1} \alpha\right)}{\exp \left(-\lambda_{2} \alpha\right)} \\
& =\left(\frac{\gamma}{1-\gamma}\right) \frac{\exp \left(-\lambda_{1} \alpha\right)}{\exp \left(-\lambda_{2} \alpha\right)}
\end{aligned}
$$

Solving Eqn. Eqn 7.6 for a mixture of candidate light nuclei and candidate heavy nuclei would allow one to determine $\alpha$, which is a suitable break point value to analyze the lighter particle (in mass) contribution to the overall distribution. Results of a sample solution of the equation for $\beta=0.90$ and $E=10^{18.5} \mathrm{eV}$ are shown in Figs. 7.2-7.6. Among the chosen hadronic interaction models, the mean-free paths do not change drastically with energy (see Table 6.3). Thus, the same values computed for $\mathrm{E}=10^{18.75} \mathrm{eV}$ will be used throughout the analysis. 


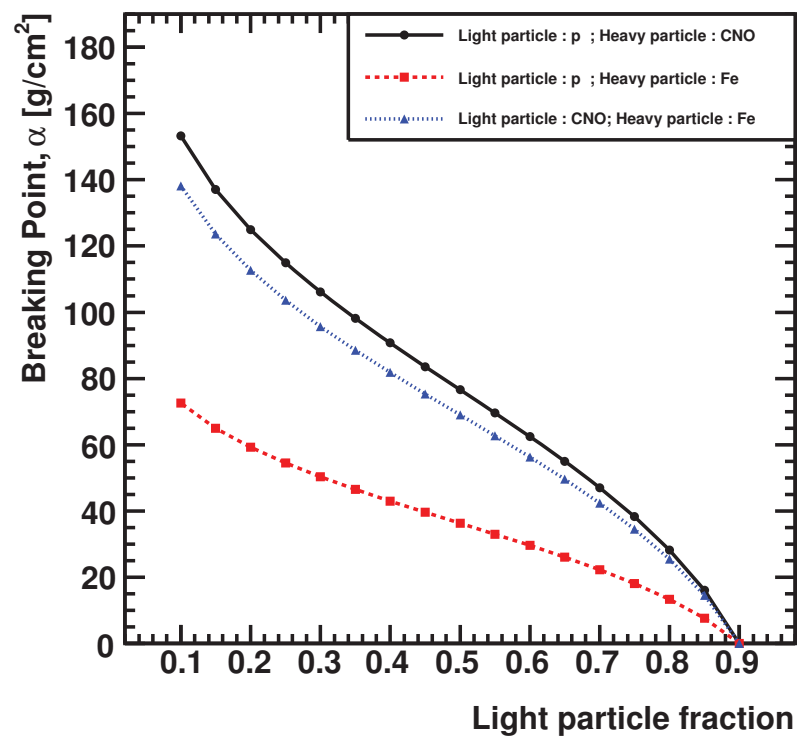

Figure 7.2: Solution of Eqn.Eqn 7.6 for $\alpha$ for p-CNO (black), p-Fe (red), CNO-Fe (blue) mixtures for different mixture ratios $(\beta=0.90)$

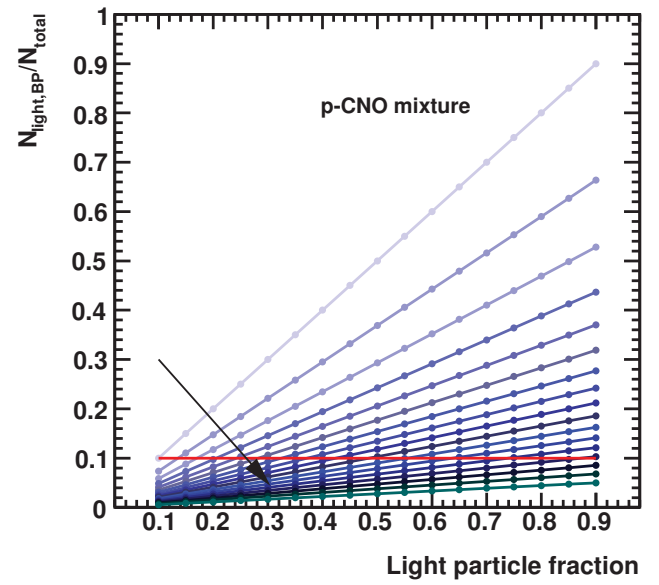

(a) Fraction of light particle after the break point with respect to the overall distribution for a given break point

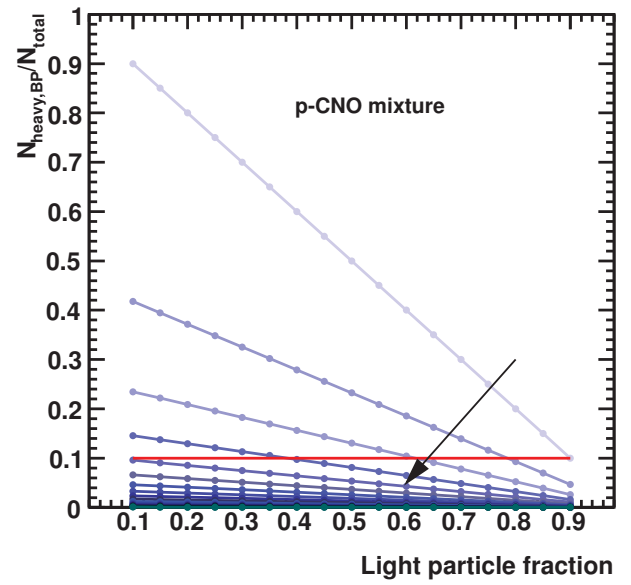

(b) Fraction of heavy particle after the break point with respect to the overall distribution for a given break point

Figure 7.3: Fraction of particles after a selected break point for different light particle fraction. Proton is used for light particles and $\mathrm{CNO}$ are used for heavy particles. Break point on each individual line is the solution of Eqn. Eqn 7.6 for a certain light particle fraction. The arrows indicate the decrease of light particle fraction used for the calculation of the break point. $(\beta=0.90)$ 


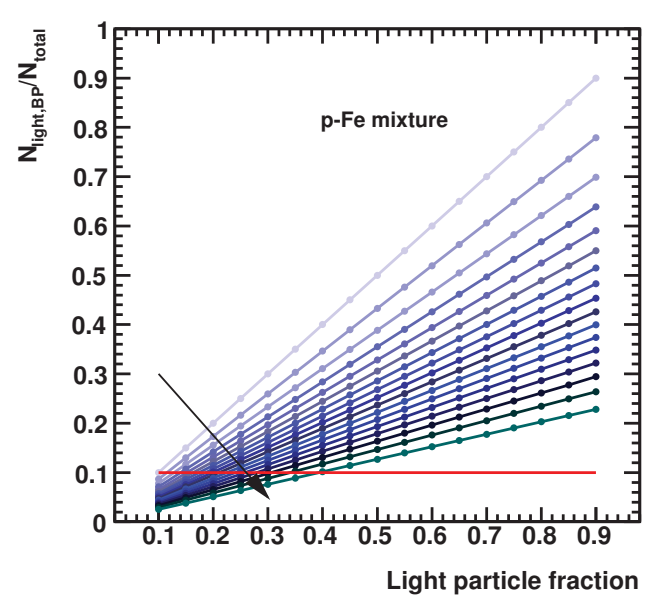

(a) Fraction of light particle after the break point with respect to the overall distribution for a given break point

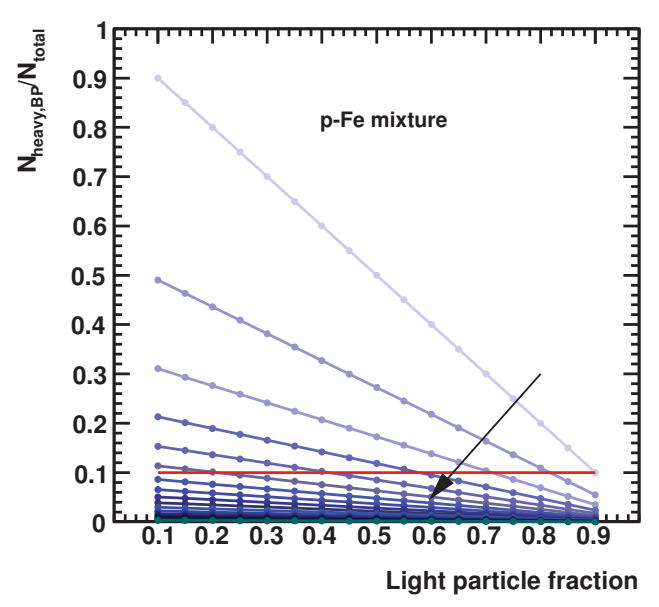

(b) Fraction of heavy particle after the break point with respect to the overall distribution for a given break point

Figure 7.4: Fraction of particles after a selected break point for different light particle fraction. Proton is used for light particles and Fe is used for heavy particles. Break point on each individual line is the solution of Eqn. Eqn 7.6 for a certain light particle fraction. The arrows indicate the decrease of light particle fraction used for the calculation of the break point. $(\beta=0.90)$

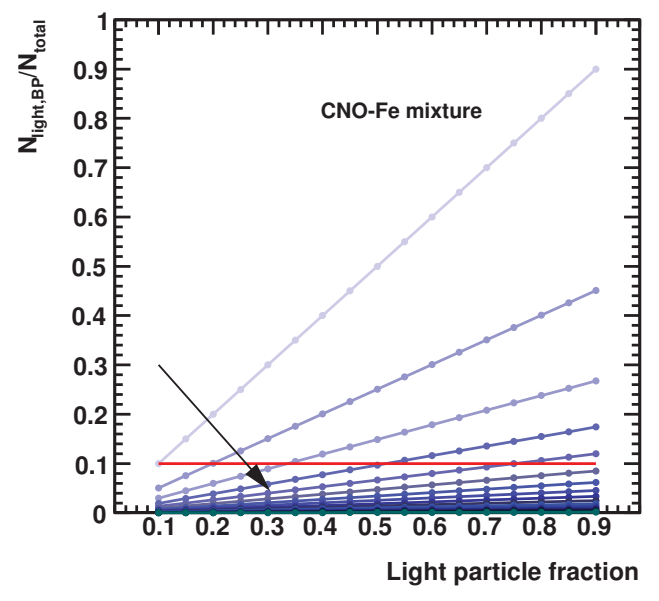

(a) Fraction of light particle after the break point with respect to the overall distribution for a given break point

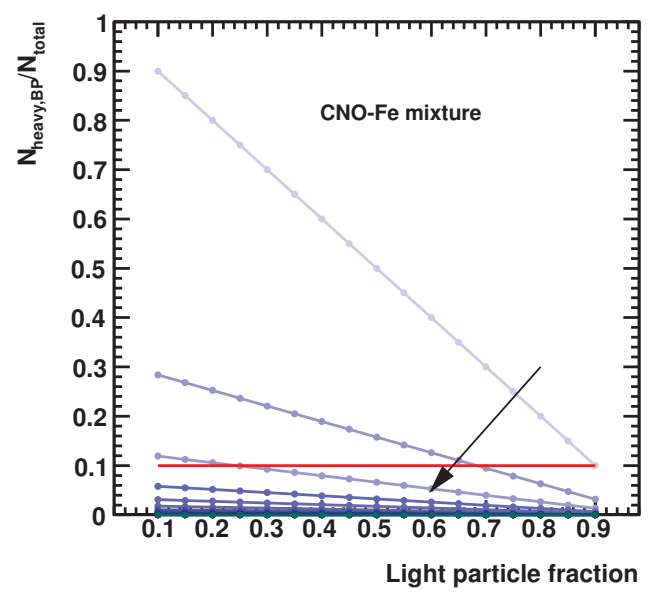

(b) Fraction of heavy particle after the break point with respect to the overall distribution for a given break point

Figure 7.5: Fraction of particles after a selected break point for different light particle fraction. $\mathrm{CNO}$ are used for light particles and $\mathrm{Fe}$ is used for heavy particles. Break point on each individual line is the solution of Eqn. Eqn 7.6 for a certain light particle fraction. The arrows indicate the decrease of light particle fraction used for the calculation of the break point. $(\beta=0.90)$ 


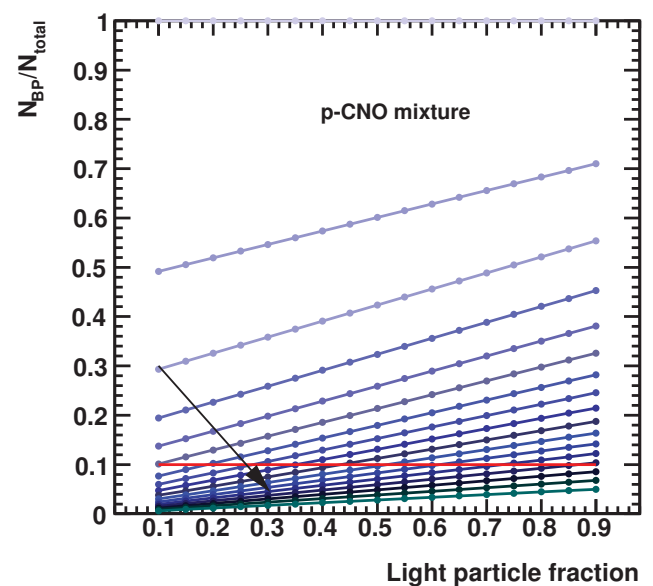

(a)

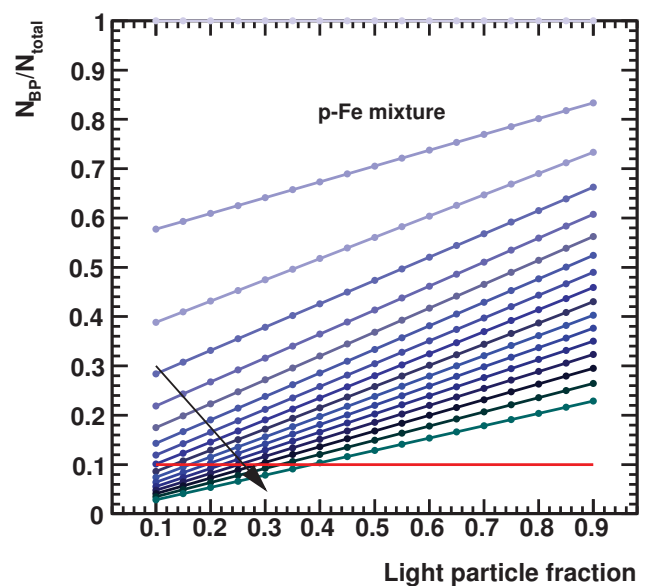

(b)

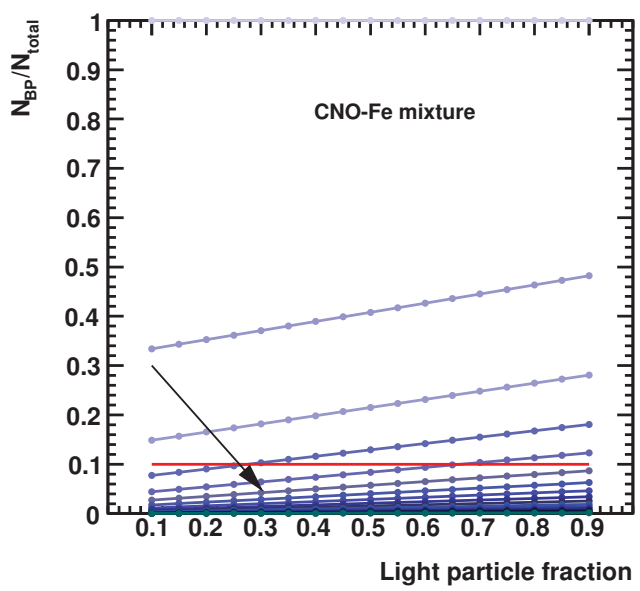

(c)

Figure 7.6: Fraction of particles (both light and heavy particles) after a selected break point for different light particle fraction for (a) p-CNO mixture, (b) p-Fe mixture, (c) CNO-Fe mixture. Break point on each individual line is the solution of Eqn. Eqn 7.6 for a certain light particle fraction. The arrows indicate the decrease of light particle fraction used for the calculation of the break point. $(\beta=0.90)$ 


\subsection{Computing break points for a two-component simulated dataset}

Given $\gamma$ and $\beta$ values, Eq. Eqn 7.6 can be solved for different ratios of nuclei as described in Sec. 7.1. Despite this fact, the actual ratios of nuclei in cosmic rays are not known. A single break point should be determined that is going to minimize the bias in the prediction for different ratios of two nuclei. The single break point and its uncertainty can be computed through the bootstrap method incorporating Monte-Carlo Simulations. However, three different break points will be computed due to three possible mixtures of nuclei, p-CNO, CNO-Fe and p-Fe. Thus, an algorithm had to be devised to choose the right break point for a given scenario.

\subsubsection{Application of Bootstrap Method with Monte Carlo Number Generator}

As stated, the break point should segregate the first interaction length distributions irrespective of the hadronic interaction model. To remove any possible hadronic interaction model dependence, the break point analysis has been carried out with Monte Carlo Simulations.

The distribution of first interaction lengths follows an exponential distribution. Applying "Inverse Method" for Monte Carlo Number Generator, values for $\mathbf{X}_{1}^{*}$ i can be computed using the equation below (Eqn. Eqn 7.7):

$$
X_{1}^{j *}=-\frac{1}{\lambda_{k}} \times \log (x) \quad \mathrm{j}=1,2, \ldots, \mathrm{N}
$$

where $\lambda_{k}$ is the expected mean-free path retrieved from hadronic interaction models with $\mathrm{k}$ being proton, $\mathrm{CNO}$ or iron and $\mathrm{x}$ is a random number in $[0,1)$. Thus, for any set, for a chosen fraction for primaries, different sets could be generated. For each set, using break point, $\alpha$, for a selected $\gamma$ and $\beta$ corresponding $\lambda^{*}$ was calculated. Through the distribution of $\lambda_{i}^{*}$, the mean and uncertainty values for $\lambda$ for the given set were computed. 


\subsection{Testing the Break Points}

Having found the BPs, they were tested for mixed composition scenarios for different hadronic interaction models. The bootstrap method was applied in order to determine the systematic errors of the predictions. Since the analytical solution is of importance, the events to be run with the bootstrap method were generated with a simple Monte-Carlo Number Generator rather than EAS simulations.

The number of events could be chosen to be any number while using Monte-Carlo simulations; however, to be realistic and close to the number of showers in EAS simulations database, 2000 events were generated for each repetition of the bootstrap method. The number of repetitions was decided upon with an iterative process. Starting with a small number of repetitions for a given case, the number of repetitions was increased until the change in variances of $\overline{X_{1}}$ is small [90]. In addition, the procedure was terminated at a maximum of 2000 repetitions.

The analysis was carried out for different hadronic interaction models, separately. For QGSJET Monte Carlo Number Generator, expected mean free paths for proton, CNO and iron were $41.00 \mathrm{~g} / \mathrm{cm}^{2}, 17.77 \mathrm{~g} / \mathrm{cm}^{2}$ and $10.26 \mathrm{~g} / \mathrm{cm}^{2}$, respectively. Similarly, for SIBYLL, $35.40 \mathrm{~g} / \mathrm{cm}^{2}, 15.05 \mathrm{~g} / \mathrm{cm}^{2}$ and $9.28 \mathrm{~g} / \mathrm{cm}^{2}$ were entered for proton, CNO and iron, correspondingly. Lastly, for EPOS, the values were $39.34 \mathrm{~g} / \mathrm{cm}^{2}, 16.17 \mathrm{~g} / \mathrm{cm}^{2}$ and $10.05 \mathrm{~g} / \mathrm{cm}^{2}$ for proton, $\mathrm{CNO}$ and iron.

\subsubsection{Testing p-CNO composition}

The results of two sample proton-CNO mixtures for QGSJET, SIBYLL and EPOS are shown in Figs. 7.7, 7.8, 7.9, respectively. The former figures demonstrate the change in the calculated value of proton for different $\beta$ values (on x-axis) and $\gamma$ values (different color codes). The shaded area indicates the simulation value of the mean free path of the primary particle. The average error for the calculated values was shown as the error bar at $\beta=1$.0. 


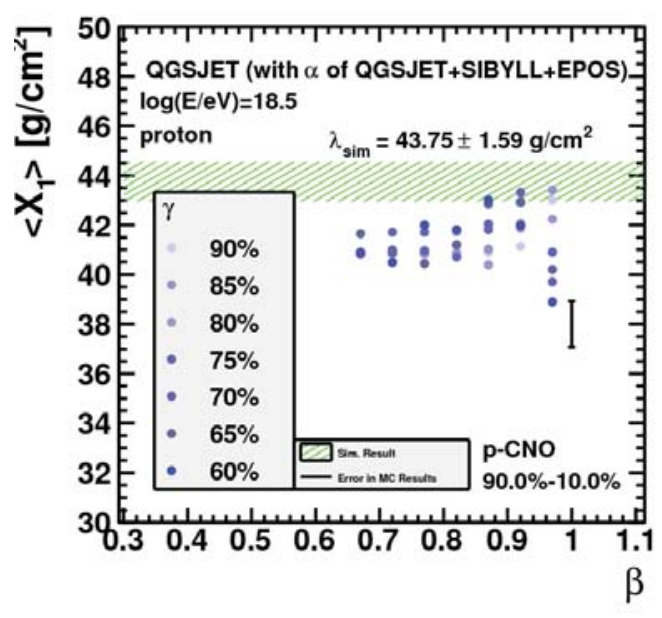

(a)

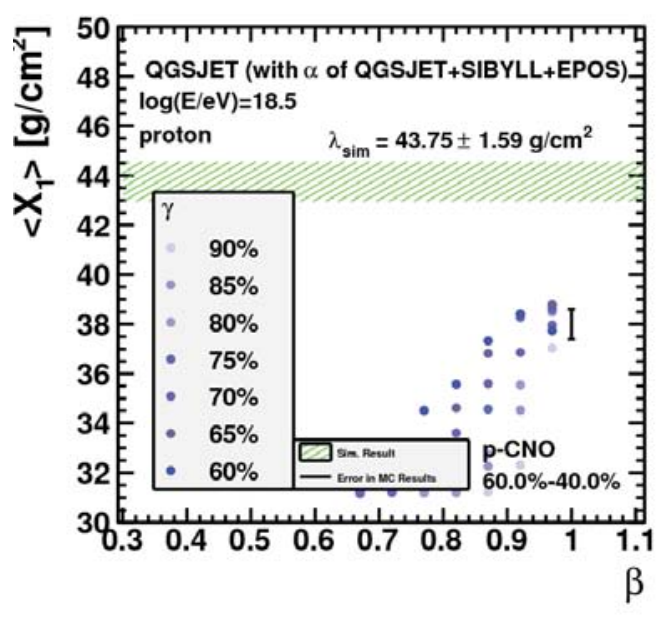

(b)

Figure 7.7: Results of bootstrap analysis for different $\beta$ and $\gamma$ values applied to QGSJET simulations. Results for proton first interaction length are shown in (a) for $90 \%$ proton $-10 \% \mathrm{CNO}$ mixture and (b) for $60 \%$ proton - $40 \% \mathrm{CNO}$ mixture.

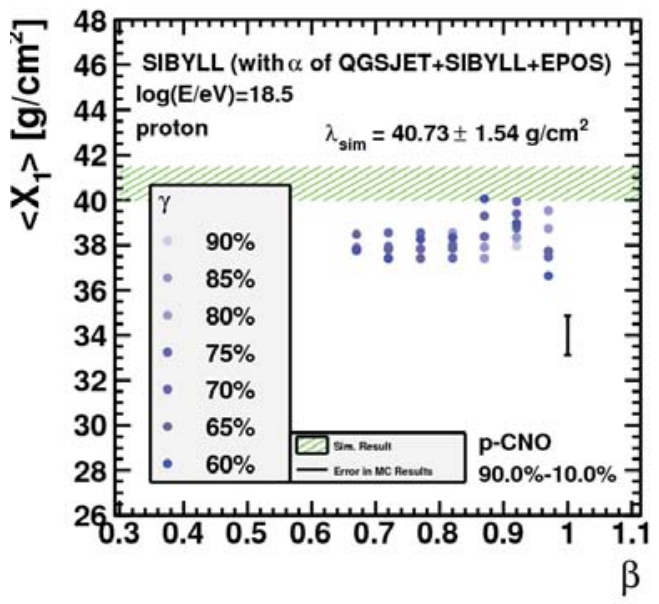

(a)

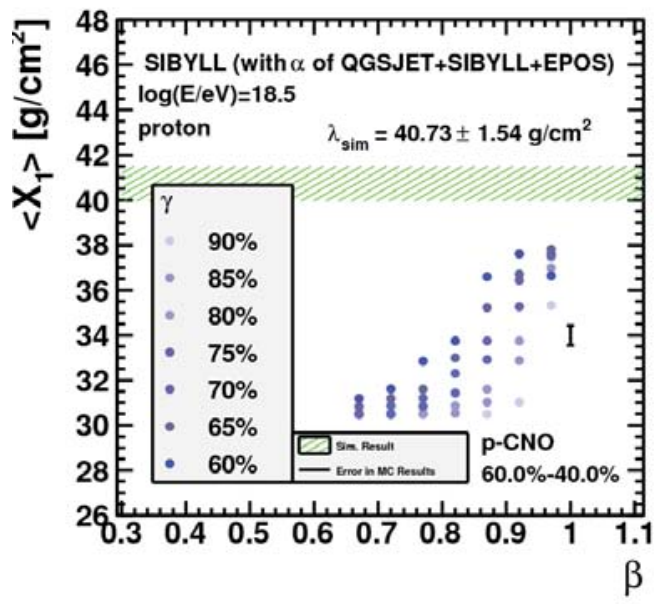

(b)

Figure 7.8: Results of bootstrap analysis for different $\beta$ and $\gamma$ values applied to SIBYLL simulations. Results for proton first interaction length are shown in (a) for $90 \%$ proton $-10 \%$ CNO mixture and (b) for $60 \%$ proton - $40 \%$ CNO mixture. 


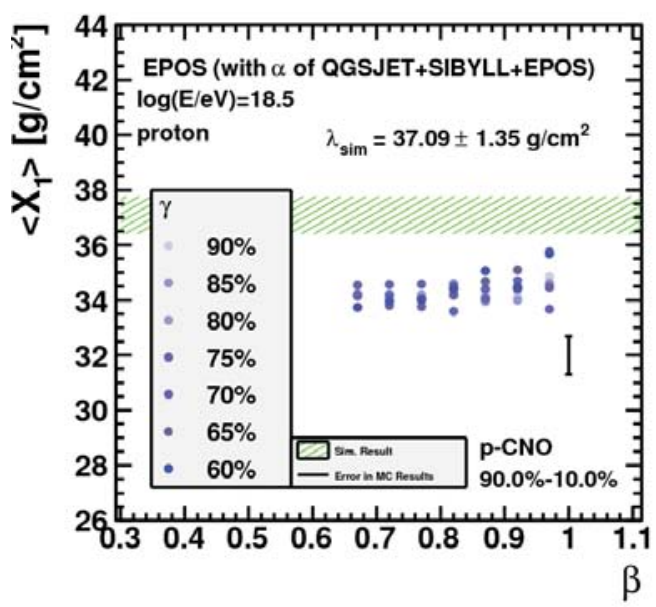

(a)

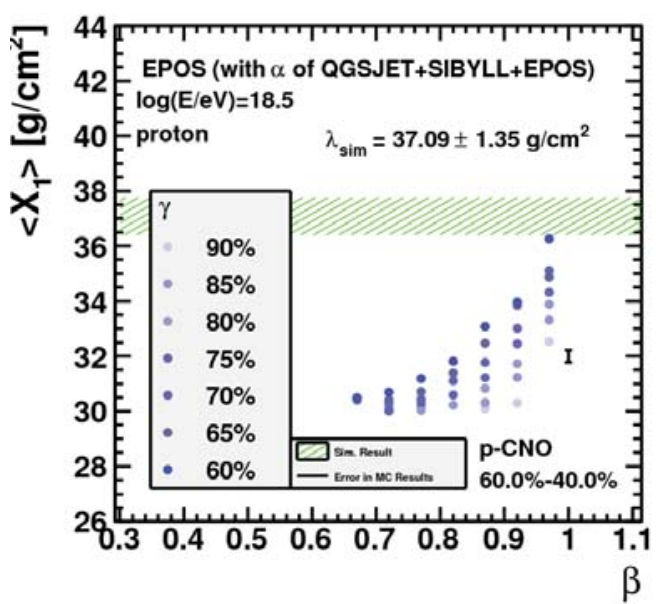

(b)

Figure 7.9: Results of bootstrap analysis for different $\beta$ and $\gamma$ values applied to EPOS simulations. Results for proton first interaction length are shown in (a) for $90 \%$ proton - $10 \%$ CNO mixture and (b) for $60 \%$ proton $-40 \%$ CNO mixture.

Through the BP analysis, BP's corresponding to different $\gamma$ and $\beta$ values were tested for different mixture ratios of proton and CNO. The discrepancies between the simulation values and the calculated values via BP's for protons are shown in Figs. 7.10(a), 7.11(a), 7.12(a) in color scale. The lower discrepancies were shown as darker colors. Similarly, for $\mathrm{CNO}$, discrepancies were shown in Figs. 7.10(b), 7.11(b), 7.12(b). Values close to zero were shown as the background color of the figures. The best choice of break point would be between $60-85 \mathrm{~g} / \mathrm{cm}^{2}$. Detailed tables for the break points falling into the range were tabulated in Tables 7.1, 7.2 and 7.3. To minimize the error for all three models, 70.12 $\mathrm{g} / \mathrm{cm}^{2}$ was selected as the break point for proton and $\mathrm{CNO}$ mixture.

The tail of first interaction length distribution composed of two components disappears for low fractions of the light particle making up the composition (See Figure 7.1). Thus, as the proton fraction decreases, the fraction after the BP would be helpful to switch to other composition scenarios from p-CNO scenario (Figs. 7.10(c), 7.11(c), 7.12(c)). Through the examination of Tables 7.1, 7.2 and 7.3, the fraction after the BP for switching was selected as $7 \%$. 


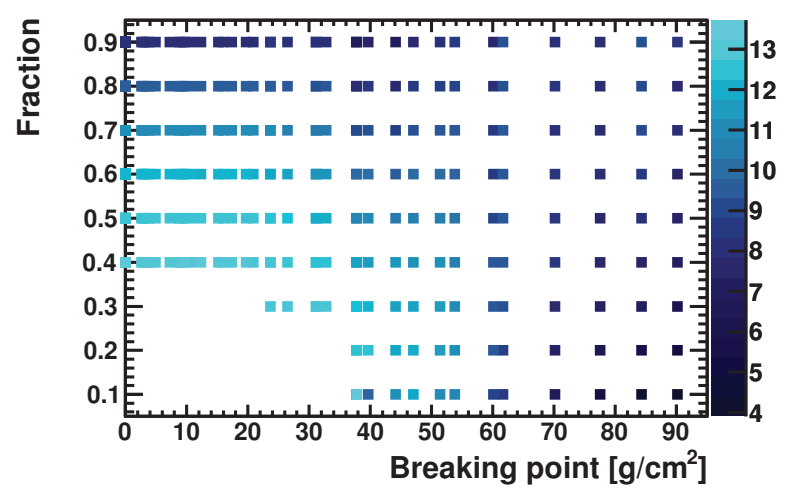

(a)

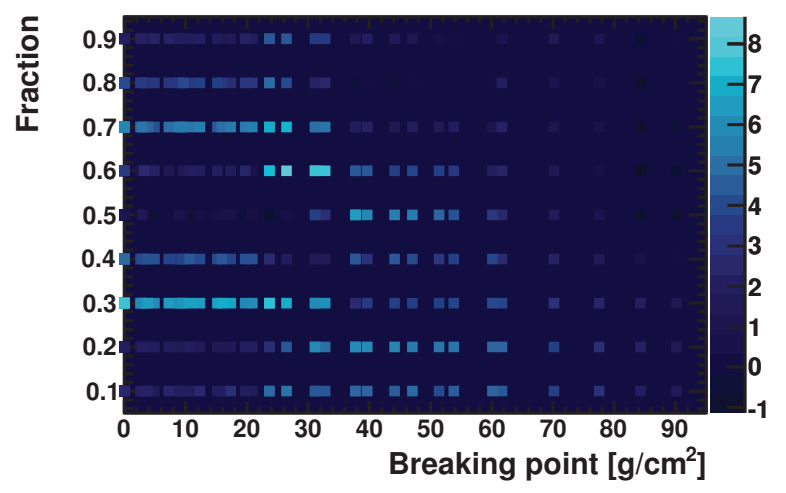

(b)

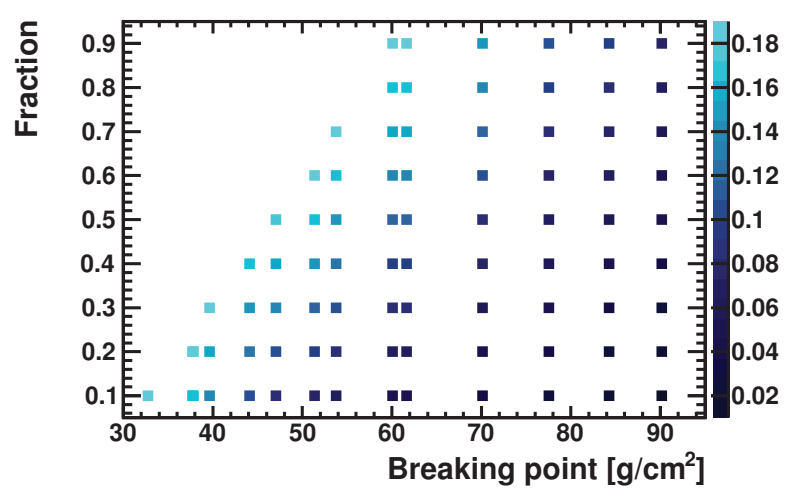

(c)

Figure 7.10: Results of different break points for different proton and CNO mixtures using QGSJET interaction model values. y-axis is the fraction of proton in the mixture. Color codes indicate the difference of calculated mean free path and the simulation value for (a) proton and (b) for $\mathrm{CNO}$, and (c) fraction of data after the break point for the first interaction length distributions. Data points out of the range are not plotted. 


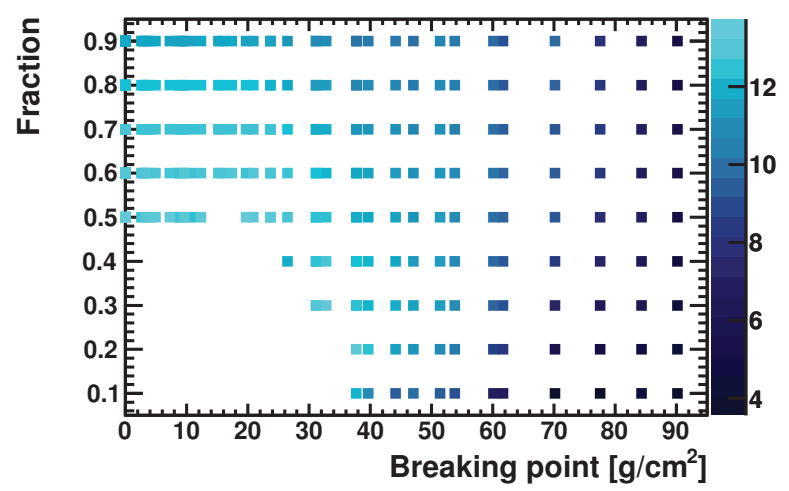

(a)

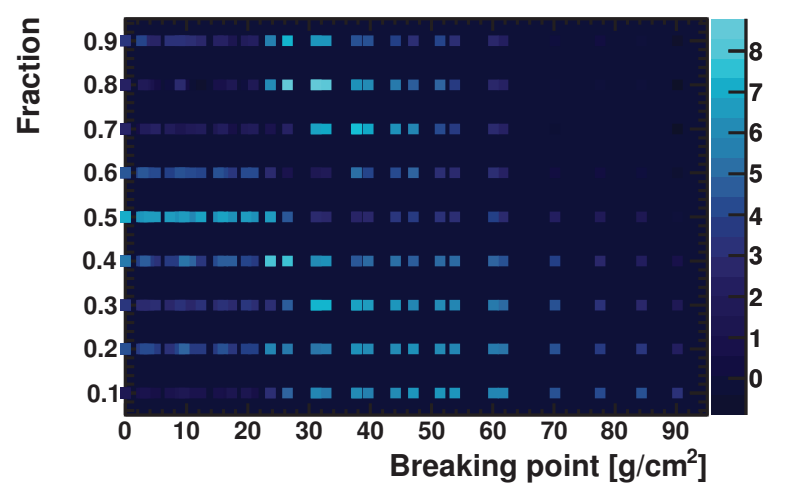

(b)

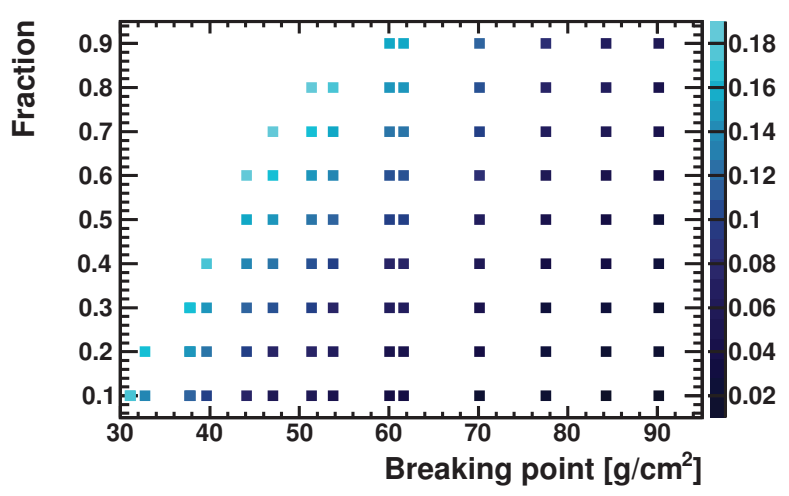

(c)

Figure 7.11: Results of different break points for different proton and $\mathrm{CNO}$ mixtures using SIBYLL interaction model values. $y$-axis is the fraction of proton in the mixture. Color codes indicate the difference of calculated mean free path and the simulation value (a) for proton and (b) for $\mathrm{CNO}$, and (c) fraction of data after the break point for the first interaction length distributions. Data points out of the range are not plotted. 


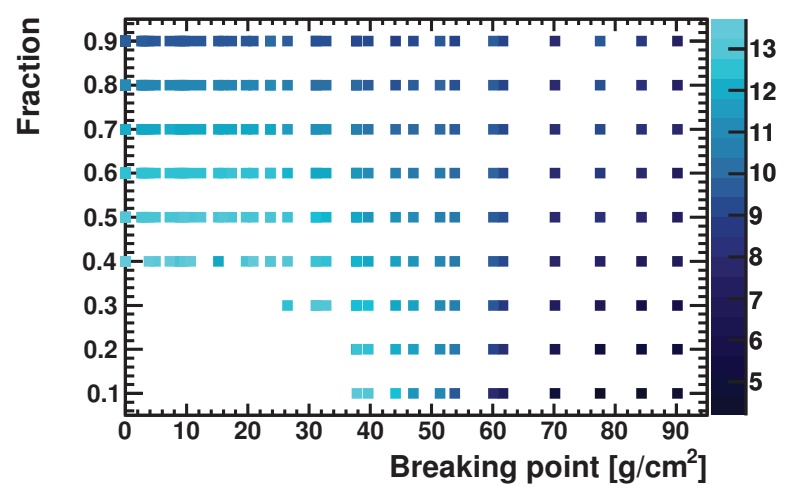

(a)

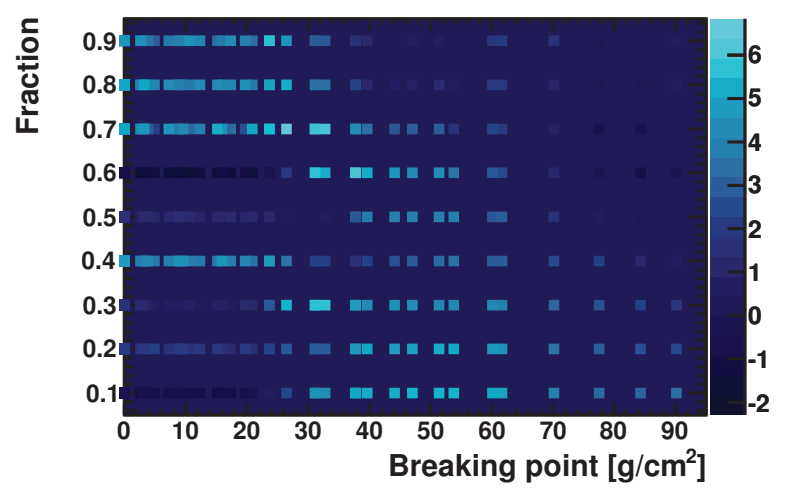

(b)

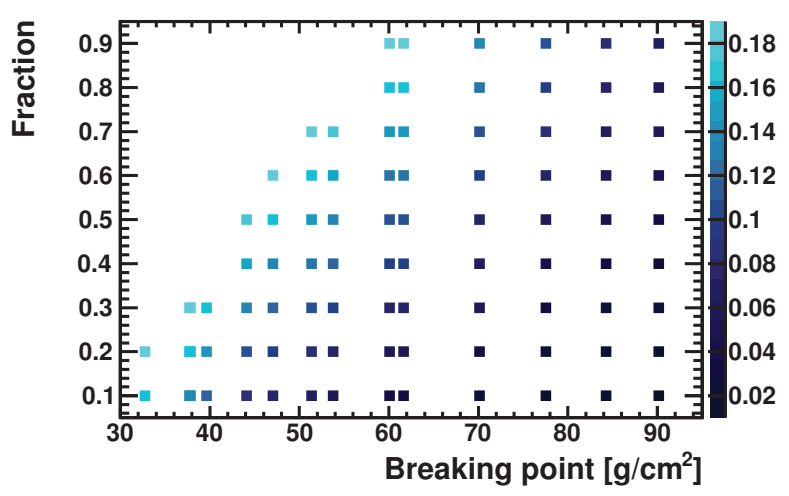

(c)

Figure 7.12: Results of different break points for different proton and CNO mixtures using EPOS interaction model values. $y$-axis is the fraction of proton in the mixture. Color codes indicate the difference of calculated mean free path and the simulation value (a) for proton and (b) for CNO, and (c) fraction of data after the break point for the first interaction length distributions. Data points out of the range are not plotted. 
Table 7.1: Results of different breaking points for various fractions of proton CNO mixture. Mean free path values of proton and $\mathrm{p}$ were computed with Monte Carlo Number Generator fed by QGSJET values. Differences were calculated using the simulation values

\begin{tabular}{|c|c|c|c|c|c|c|}
\hline $\mathrm{f}$ & $\begin{array}{c}\mathrm{BP} \\
{\left[\mathrm{g} / \mathrm{cm}^{2}\right]}\end{array}$ & $\begin{array}{l}\langle\lambda\rangle_{p, c a l} \\
{\left[\mathrm{~g} / \mathrm{cm}^{2}\right]}\end{array}$ & $\begin{array}{c}\langle\lambda\rangle_{C N O, \text { cal }} \\
{\left[\mathrm{g} / \mathrm{cm}^{2}\right]}\end{array}$ & $\mathrm{f}_{B P}$ & $\begin{array}{c}\langle\lambda\rangle_{p, \text { sim }}-\langle\lambda\rangle_{p, \text { cal }} \\
{\left[\mathrm{g} / \mathrm{cm}^{2}\right]}\end{array}$ & $\begin{array}{c}\langle\lambda\rangle_{C N O, \text { sim }}-\langle\lambda\rangle_{C N O, \text { cal }} \\
{\left[\mathrm{g} / \mathrm{cm}^{2}\right]}\end{array}$ \\
\hline 0.90 & 84.27 & 34.29 & 18.01 & 0.10 & 6.71 & -0.24 \\
\hline 0.80 & 84.27 & 34.50 & 18.48 & 0.09 & 6.50 & -0.71 \\
\hline 0.70 & 84.27 & 34.47 & 18.54 & 0.08 & 6.53 & -0.77 \\
\hline 0.60 & 84.27 & 35.00 & 18.85 & 0.07 & 6.00 & -1.08 \\
\hline 0.50 & 84.27 & 35.38 & 18.25 & 0.06 & 5.62 & -0.48 \\
\hline 0.40 & 84.27 & 36.00 & 16.90 & 0.05 & 5.00 & 0.87 \\
\hline 0.30 & 84.27 & 36.59 & 15.43 & 0.04 & 4.41 & 2.34 \\
\hline 0.20 & 84.27 & 37.76 & 15.27 & 0.03 & 3.24 & 2.50 \\
\hline 0.10 & 84.27 & 39.55 & 15.41 & 0.02 & 1.45 & 2.36 \\
\hline$\overline{0.90}$ & 77.54 & 35.59 & 16.50 & 0.11 & 5.41 & 1.27 \\
\hline 0.80 & 77.54 & 35.53 & 16.92 & 0.10 & 5.47 & 0.85 \\
\hline 0.70 & 77.54 & 35.49 & 16.84 & 0.09 & 5.51 & 0.93 \\
\hline$\overline{0.60}$ & 77.54 & 35.55 & 17.09 & 0.08 & 5.45 & 0.68 \\
\hline 0.50 & 77.54 & 35.94 & 16.63 & 0.07 & 5.06 & 1.14 \\
\hline 0.40 & 77.54 & 35.96 & 16.00 & 0.06 & 5.04 & 1.77 \\
\hline 0.30 & 77.54 & 36.33 & 15.02 & 0.05 & 4.67 & 2.75 \\
\hline 0.20 & 77.54 & 37.25 & 14.50 & 0.04 & 3.75 & 3.27 \\
\hline 0.10 & 77.54 & 38.04 & 14.47 & 0.03 & 2.96 & 3.30 \\
\hline 0.90 & 70.12 & 35.44 & 16.08 & 0.15 & 5.56 & 1.69 \\
\hline 0.80 & 70.12 & 35.25 & 16.59 & 0.14 & 5.75 & 1.18 \\
\hline 0.70 & 70.12 & 35.30 & 16.54 & 0.12 & 5.70 & 1.23 \\
\hline 0.60 & 70.12 & 35.13 & 16.40 & 0.11 & 5.87 & 1.37 \\
\hline 0.50 & 70.12 & 35.16 & 15.94 & 0.09 & 5.84 & 1.83 \\
\hline 0.40 & 70.12 & 35.24 & 15.28 & 0.08 & 5.76 & 2.49 \\
\hline 0.30 & 70.12 & 35.66 & 14.43 & 0.06 & 5.34 & 3.34 \\
\hline 0.20 & 70.12 & 36.33 & 13.87 & 0.05 & 4.67 & 3.90 \\
\hline 0.10 & 70.12 & 36.98 & 13.81 & 0.04 & 4.02 & 3.96 \\
\hline 0.90 & 61.64 & 34.41 & 16.62 & 0.19 & 6.59 & 1.15 \\
\hline$\overline{0.80}$ & 61.64 & 34.24 & 15.70 & 0.17 & 6.76 & 2.07 \\
\hline 0.70 & 61.64 & 34.17 & 15.34 & 0.16 & 6.83 & 2.43 \\
\hline 0.60 & 61.64 & 34.07 & 15.10 & 0.14 & 6.93 & 2.67 \\
\hline 0.50 & 61.64 & 34.18 & 14.85 & 0.12 & 6.82 & 2.92 \\
\hline 0.40 & 61.64 & 34.17 & 14.73 & 0.10 & 6.83 & 3.04 \\
\hline 0.30 & 61.64 & 34.34 & 13.77 & 0.09 & 6.66 & 4.00 \\
\hline 0.20 & 61.64 & 34.81 & 12.90 & 0.07 & 6.19 & 4.87 \\
\hline 0.10 & 61.64 & 35.01 & 13.08 & 0.05 & 5.99 & 4.69 \\
\hline
\end{tabular}


Table 7.2: Results of different breaking points for various fractions of proton CNO mixture. Mean free path values of proton and CNO were computed with Monte Carlo Number Generator fed by SIBYLL values. Differences were calculated using the simulation values

\begin{tabular}{|c|c|c|c|c|c|c|}
\hline $\mathrm{f}$ & $\begin{array}{c}\mathrm{BP} \\
{\left[\mathrm{g} / \mathrm{cm}^{2}\right]}\end{array}$ & $\begin{array}{l}\langle\lambda\rangle_{p, c a l} \\
{\left[\mathrm{~g} / \mathrm{cm}^{2}\right]}\end{array}$ & $\begin{array}{c}\langle\lambda\rangle_{C N O, \text { cal }} \\
{\left[\mathrm{g} / \mathrm{cm}^{2}\right]}\end{array}$ & $\mathrm{f}_{B P}$ & $\begin{array}{c}\langle\lambda\rangle_{p, \text { sim }}-\langle\lambda\rangle_{p, \text { cal }} \\
{\left[\mathrm{g} / \mathrm{cm}^{2}\right]}\end{array}$ & $\begin{array}{c}\langle\lambda\rangle_{C N O, \text { sim }}-\langle\lambda\rangle_{C N O, \text { cal }} \\
{\left[\mathrm{g} / \mathrm{cm}^{2}\right]}\end{array}$ \\
\hline 0.90 & 84.27 & 36.80 & 17.42 & 0.07 & -1.40 & -2.37 \\
\hline 0.80 & 84.27 & 36.83 & 17.54 & 0.07 & -1.43 & -2.49 \\
\hline 0.70 & 84.27 & 36.62 & 17.66 & 0.06 & -1.22 & -2.61 \\
\hline 0.60 & 84.27 & 36.76 & 17.30 & 0.05 & -1.36 & -2.25 \\
\hline 0.50 & 84.27 & 36.70 & 16.18 & 0.04 & -1.30 & -1.13 \\
\hline 0.40 & 84.27 & 37.26 & 15.38 & 0.04 & -1.86 & -0.33 \\
\hline 0.30 & 84.27 & 37.58 & 14.67 & 0.03 & -2.18 & 0.38 \\
\hline 0.20 & 84.27 & 38.70 & 14.04 & 0.02 & -3.30 & 1.01 \\
\hline 0.10 & 84.27 & 38.82 & 13.18 & 0.01 & -3.42 & 1.87 \\
\hline$\overline{0.90}$ & 77.54 & 35.55 & 17.15 & 0.09 & -0.15 & -2.10 \\
\hline 0.80 & 77.54 & 35.35 & 17.56 & 0.08 & 0.05 & -2.51 \\
\hline 0.70 & 77.54 & 35.26 & 17.58 & 0.07 & 0.14 & -2.53 \\
\hline 0.60 & 77.54 & 35.44 & 17.11 & 0.06 & -0.04 & -2.06 \\
\hline 0.50 & 77.54 & 35.80 & 15.78 & 0.05 & -0.40 & -0.73 \\
\hline 0.40 & 77.54 & 36.34 & 14.69 & 0.04 & -0.94 & 0.36 \\
\hline 0.30 & 77.54 & 37.31 & 14.20 & 0.03 & -1.91 & 0.85 \\
\hline 0.20 & 77.54 & 38.46 & 13.80 & 0.03 & -3.06 & 1.25 \\
\hline 0.10 & 77.54 & 40.19 & 13.39 & 0.02 & -4.79 & 1.66 \\
\hline 0.90 & 70.12 & 33.77 & 17.23 & 0.12 & 1.63 & -2.18 \\
\hline 0.80 & 70.12 & 33.92 & 17.57 & 0.11 & 1.48 & -2.52 \\
\hline 0.70 & 70.12 & 34.26 & 17.86 & 0.10 & 1.14 & -2.81 \\
\hline 0.60 & 70.12 & 34.13 & 17.20 & 0.09 & 1.27 & -2.15 \\
\hline 0.50 & 70.12 & 34.31 & 15.29 & 0.07 & 1.09 & -0.24 \\
\hline 0.40 & 70.12 & 35.17 & 13.72 & 0.06 & 0.23 & 1.33 \\
\hline 0.30 & 70.12 & 36.23 & 13.24 & 0.05 & -0.83 & 1.81 \\
\hline 0.20 & 70.12 & 37.98 & 13.17 & 0.04 & -2.58 & 1.88 \\
\hline 0.10 & 70.12 & 39.86 & 13.10 & 0.02 & -4.46 & 1.95 \\
\hline 0.90 & 61.64 & 34.42 & 15.23 & 0.16 & 0.98 & -0.18 \\
\hline$\overline{0.80}$ & 61.64 & 34.22 & 15.11 & 0.15 & 1.18 & -0.06 \\
\hline 0.70 & 61.64 & 34.07 & 15.10 & 0.13 & 1.33 & -0.05 \\
\hline 0.60 & 61.64 & 33.95 & 15.05 & 0.11 & 1.45 & 0.00 \\
\hline 0.50 & 61.64 & 33.98 & 14.51 & 0.10 & 1.42 & 0.54 \\
\hline 0.40 & 61.64 & 34.22 & 13.09 & 0.08 & 1.18 & 1.96 \\
\hline 0.30 & 61.64 & 34.51 & 12.44 & 0.07 & 0.89 & 2.61 \\
\hline 0.20 & 61.64 & 35.44 & 12.18 & 0.05 & -0.04 & 2.87 \\
\hline 0.10 & 61.64 & 37.07 & 11.66 & 0.04 & -1.67 & 3.39 \\
\hline
\end{tabular}


Table 7.3: Results of different breaking points for various fractions of proton CNO mixture. Mean free path values of proton and CNO were computed with Monte Carlo Number Generator fed by EPOS values. Differences were calculated using the simulation values

\begin{tabular}{|c|c|c|c|c|c|c|}
\hline $\mathrm{f}$ & $\begin{array}{c}\mathrm{BP} \\
{\left[\mathrm{g} / \mathrm{cm}^{2}\right]}\end{array}$ & $\begin{array}{l}\langle\lambda\rangle_{p, c a l} \\
{\left[\mathrm{~g} / \mathrm{cm}^{2}\right]}\end{array}$ & $\begin{array}{c}\langle\lambda\rangle_{C N O, \text { cal }} \\
{\left[\mathrm{g} / \mathrm{cm}^{2}\right]}\end{array}$ & $\mathrm{f}_{B P}$ & $\begin{array}{c}\langle\lambda\rangle_{p, \text { sim }}-\langle\lambda\rangle_{p, \text { cal }} \\
{\left[\mathrm{g} / \mathrm{cm}^{2}\right]}\end{array}$ & $\begin{array}{c}\langle\lambda\rangle_{C N O, \text { sim }}-\langle\lambda\rangle_{C N O, \text { cal }} \\
{\left[\mathrm{g} / \mathrm{cm}^{2}\right]}\end{array}$ \\
\hline 0.90 & 84.27 & 34.91 & 17.49 & 0.09 & 4.43 & -1.32 \\
\hline 0.80 & 84.27 & 35.17 & 17.72 & 0.08 & 4.17 & -1.55 \\
\hline 0.70 & 84.27 & 35.20 & 17.94 & 0.07 & 4.14 & -1.77 \\
\hline 0.60 & 84.27 & 35.64 & 18.35 & 0.06 & 3.70 & -2.18 \\
\hline 0.50 & 84.27 & 35.79 & 17.61 & 0.05 & 3.55 & -1.44 \\
\hline 0.40 & 84.27 & 36.65 & 16.32 & 0.04 & 2.69 & -0.15 \\
\hline 0.30 & 84.27 & 37.73 & 15.38 & 0.03 & 1.61 & 0.79 \\
\hline 0.20 & 84.27 & 38.22 & 14.90 & 0.02 & 1.12 & 1.27 \\
\hline 0.10 & 84.27 & 39.47 & 14.42 & 0.02 & -0.13 & 1.75 \\
\hline$\overline{0.90}$ & 77.54 & 34.08 & 17.58 & 0.11 & 5.26 & -1.41 \\
\hline 0.80 & 77.54 & 34.13 & 17.63 & 0.10 & 5.21 & -1.46 \\
\hline 0.70 & 77.54 & 34.46 & 17.97 & 0.09 & 4.88 & -1.80 \\
\hline$\overline{0.60}$ & 77.54 & 34.82 & 17.83 & 0.08 & 4.52 & -1.66 \\
\hline 0.50 & 77.54 & 35.18 & 16.99 & 0.06 & 4.16 & -0.82 \\
\hline 0.40 & 77.54 & 36.11 & 15.43 & 0.05 & 3.23 & 0.74 \\
\hline 0.30 & 77.54 & 36.89 & 14.89 & 0.04 & 2.45 & 1.28 \\
\hline 0.20 & 77.54 & 38.24 & 14.52 & 0.03 & 1.10 & 1.65 \\
\hline 0.10 & 77.54 & 39.56 & 14.23 & 0.02 & -0.22 & 1.94 \\
\hline 0.90 & 70.12 & 35.57 & 15.83 & 0.14 & 3.77 & 0.34 \\
\hline 0.80 & 70.12 & 35.49 & 16.48 & 0.13 & 3.85 & -0.31 \\
\hline 0.70 & 70.12 & 35.45 & 16.64 & 0.11 & 3.89 & -0.47 \\
\hline 0.60 & 70.12 & 35.54 & 16.39 & 0.10 & 3.80 & -0.22 \\
\hline 0.50 & 70.12 & 35.56 & 15.93 & 0.08 & 3.78 & 0.24 \\
\hline 0.40 & 70.12 & 35.94 & 14.97 & 0.07 & 3.40 & 1.20 \\
\hline 0.30 & 70.12 & 36.47 & 14.35 & 0.06 & 2.87 & 1.82 \\
\hline 0.20 & 70.12 & 37.32 & 13.82 & 0.04 & 2.02 & 2.35 \\
\hline 0.10 & 70.12 & 38.66 & 13.55 & 0.03 & 0.68 & 2.62 \\
\hline 0.90 & 61.64 & 34.59 & 15.54 & 0.19 & 4.75 & 0.63 \\
\hline$\overline{0.80}$ & 61.64 & 34.47 & 15.72 & 0.17 & 4.87 & 0.45 \\
\hline 0.70 & 61.64 & 34.44 & 15.55 & 0.15 & 4.90 & 0.62 \\
\hline 0.60 & 61.64 & 34.33 & 15.03 & 0.13 & 5.01 & 1.14 \\
\hline 0.50 & 61.64 & 34.14 & 14.61 & 0.11 & 5.20 & 1.56 \\
\hline 0.40 & 61.64 & 34.41 & 14.51 & 0.10 & 4.93 & 1.66 \\
\hline 0.30 & 61.64 & 35.03 & 13.55 & 0.08 & 4.31 & 2.62 \\
\hline 0.20 & 61.64 & 35.42 & 13.00 & 0.06 & 3.92 & 3.17 \\
\hline 0.10 & 61.64 & 36.54 & 12.60 & 0.04 & 2.80 & 3.57 \\
\hline
\end{tabular}




\subsubsection{Testing p-Fe composition}

As was done for the $\mathrm{p}-\mathrm{CNO}$ mixture, a similar analysis was carried out for a $\mathrm{p}$ iron mixture. BP's corresponding to different $\gamma$ and $\beta$ values were tested for different mixture ratios of proton and $\mathrm{Fe}$.

The discrepancies between the simulation values and calculated values via BP's for proton were shown in Figs. 7.16(a), 7.17(a), 7.18(a) in color scale. Darker colors show lower discrepancies. For iron, discrepancies were shown in Figs. 7.16(b), 7.16(b), 7.16(b). Colors close to background color correspond to values close to zero. The best choice of break point would be between $60-80 \mathrm{~g} / \mathrm{cm}^{2}$. Detailed tables for the break points falling into the range were tabulated in Tables 7.4, 7.5 and 7.6. To minimize the error for all three models, $70.76 \mathrm{~g} / \mathrm{cm}^{2}$ was selected as the break point for proton and iron mixture. Similar to the p-CNO composition discussion, the critical fraction below which one will assume another composition was found to be $7 \%$.

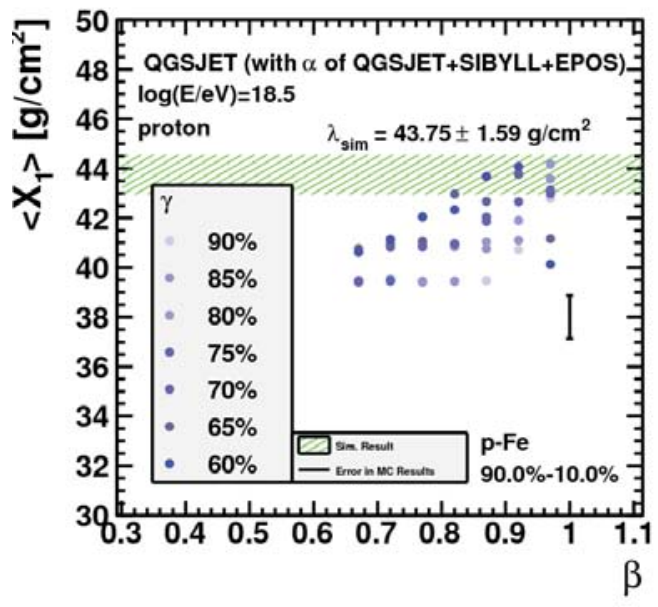

(a)

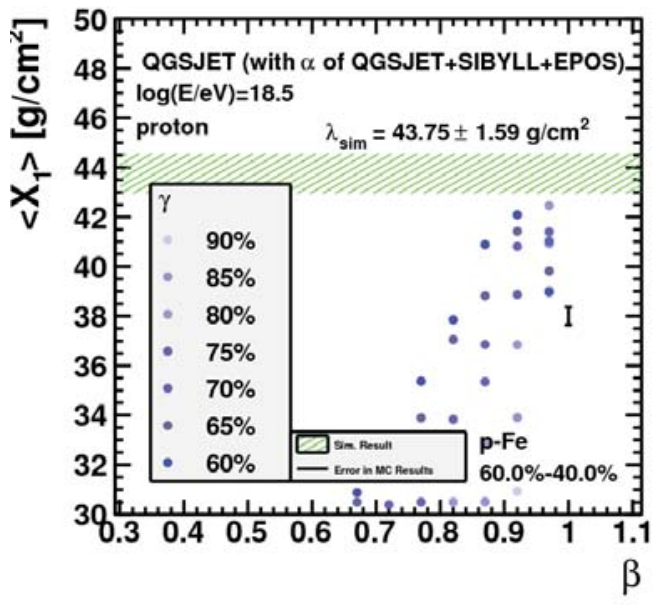

(b)

Figure 7.13: Results of bootstrap analysis for different $\beta$ and $\gamma$ values applied to QGSJET simulations. Results for proton first interaction length are shown in (a) for $90 \%$ proton - $10 \%$ iron mixture and (b) for $60 \%$ proton - $40 \%$ iron mixture. 


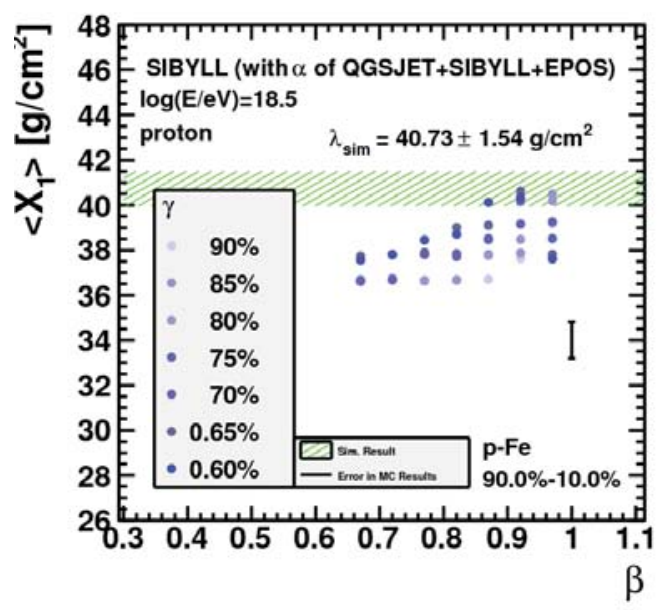

(a)

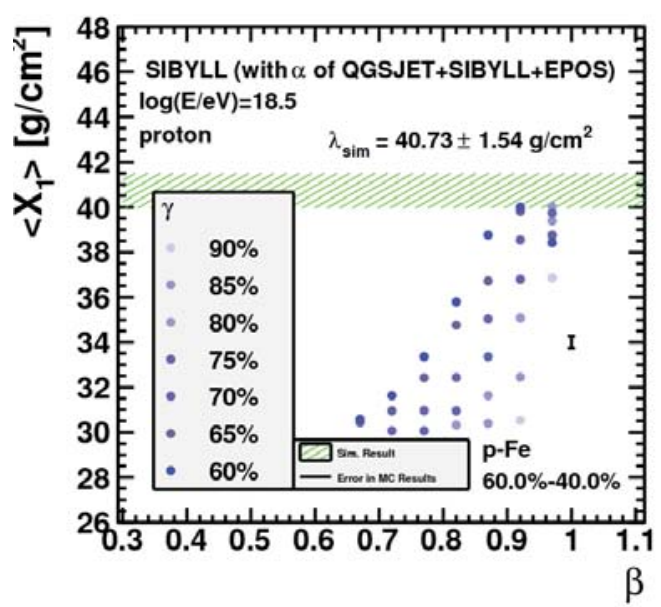

(b)

Figure 7.14: Results of bootstrap analysis for different $\beta$ and $\gamma$ values applied to SIBYLL simulations. Results for proton first interaction length are shown in (a) for $90 \%$ proton $-10 \%$ iron mixture and (b) for $60 \%$ proton - $40 \%$ iron mixture.

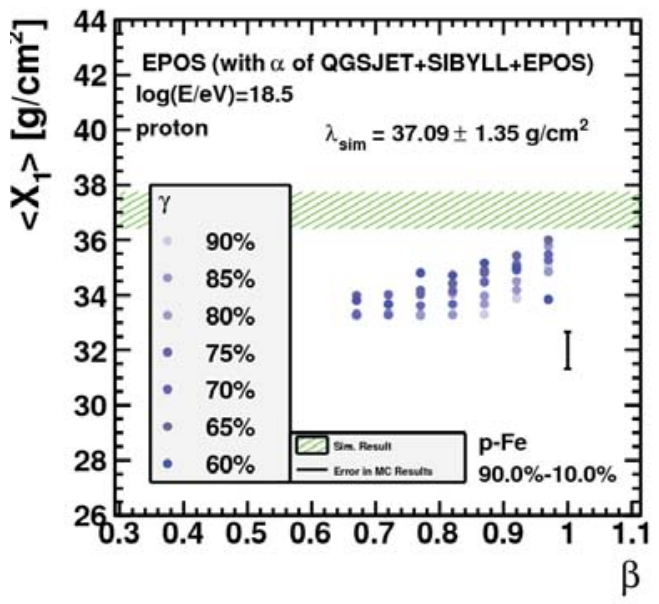

(a)

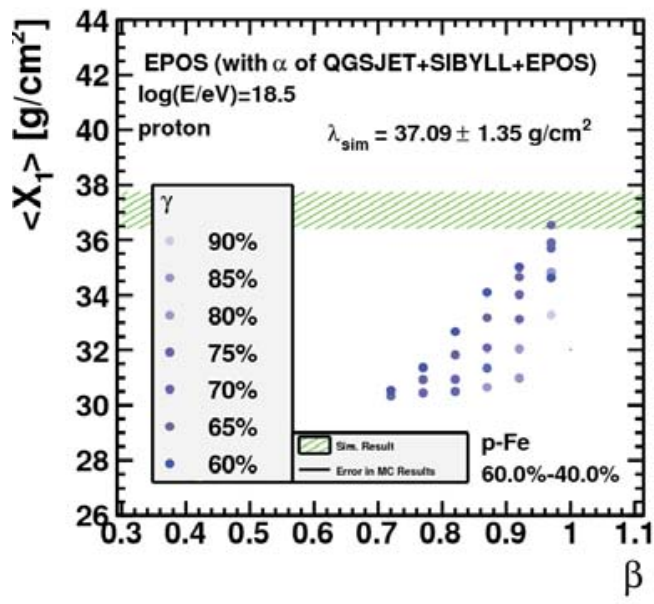

(b)

Figure 7.15: Results of bootstrap analysis for different $\beta$ and $\gamma$ values applied to EPOS simulations. Results for proton first interaction length are shown in (a) for $90 \%$ proton $-10 \%$ iron mixture and (b) for $60 \%$ proton $-40 \%$ iron mixture. 


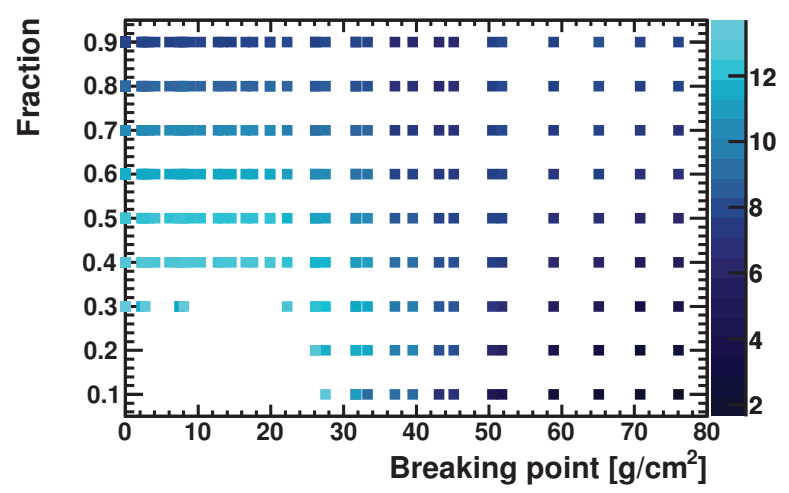

(a)

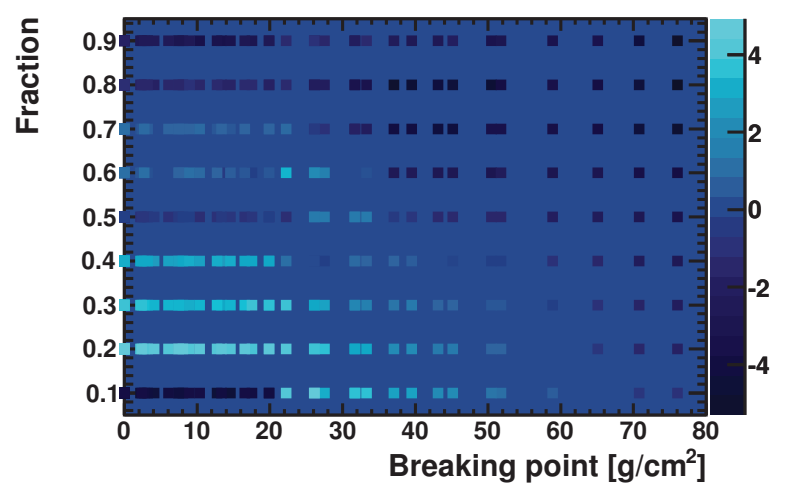

(b)

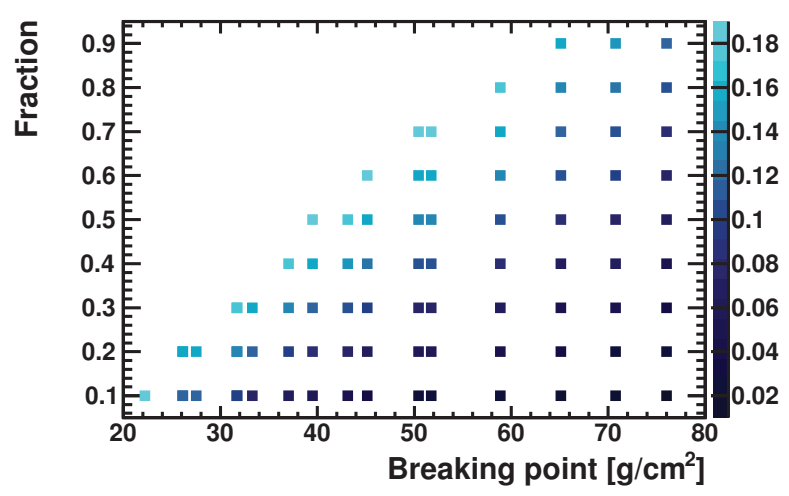

(c)

Figure 7.16: Results of different break points for different proton and iron mixtures using QGSJET interaction model values. $y$-axis is the fraction of proton in the mixture. Color codes indicate the difference of calculated mean free path and the simulation value (a) for proton and (b) for iron, and (c) fraction of data after the break point for the first interaction length distributions. Data points out of the range are not plotted. 


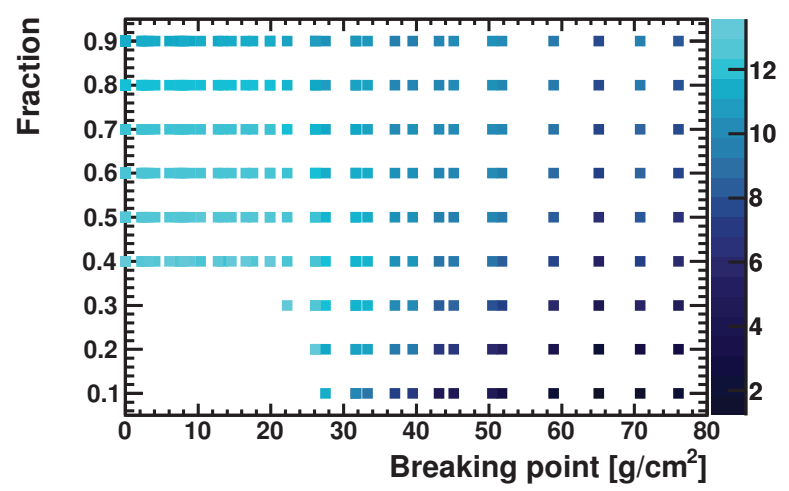

(a)

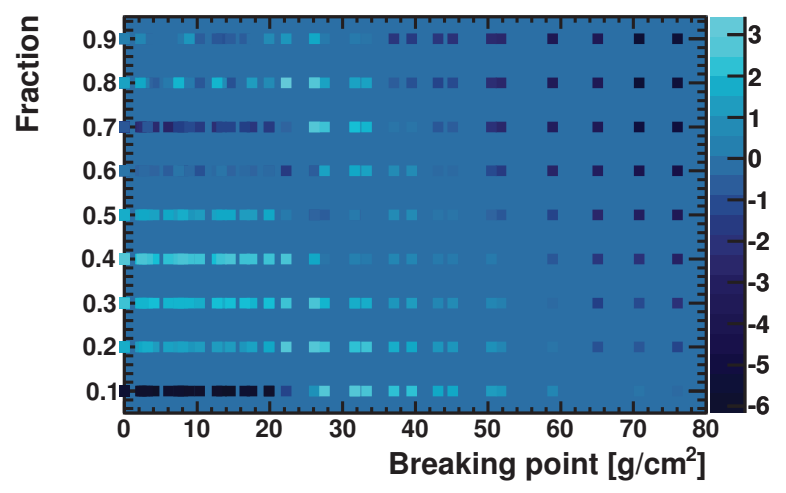

(b)

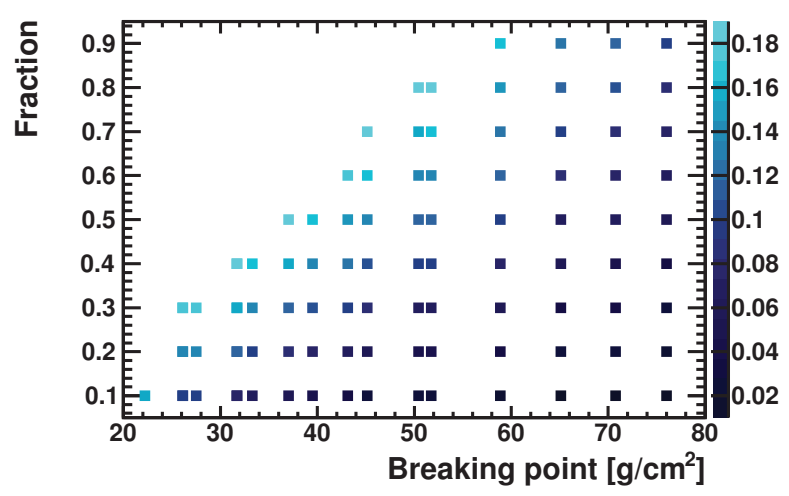

(c)

Figure 7.17: Results of different break points for different proton and iron mixtures using SIBYLL interaction model values. $y$-axis is the fraction of proton in the mixture. Color codes indicate the difference of calculated mean free path and the simulation value (a) for proton and (b) for iron, and (c) fraction of data after the break point for the first interaction length distributions. Data points out of the range are not plotted. 


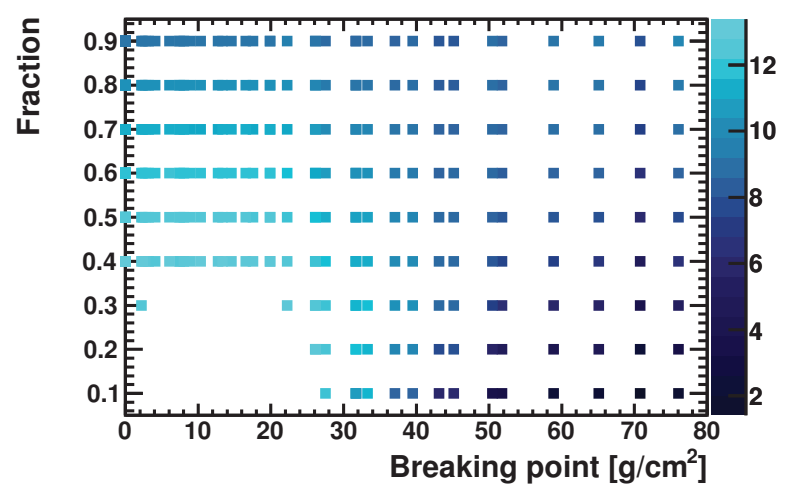

(a)

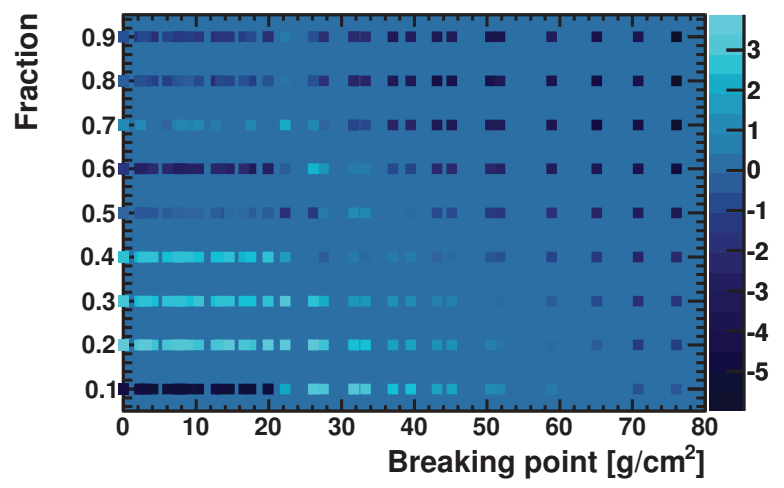

(b)

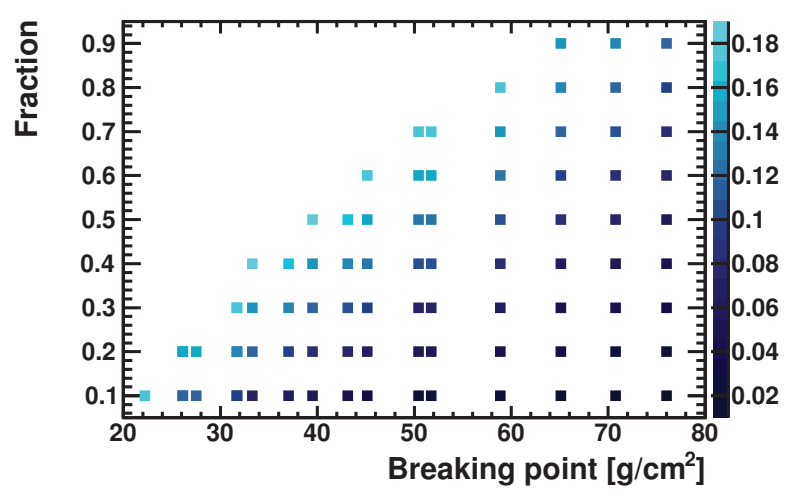

(c)

Figure 7.18: Results of different break points for different proton and iron mixtures using EPOS interaction model values. $y$-axis is the fraction of proton in the mixture. Color codes indicate the difference of calculated mean free path and the simulation value (a) for proton and (b) for iron, and (c) fraction of data after the break point for the first interaction length distributions. Data points out of the range are not plotted. 
Table 7.4: Results of different breaking points for various fractions of proton iron mixture. Mean free path values of proton and iron were computed with Monte Carlo Number Generator fed by QGSJET values. Differences were calculated using the simulation values

\begin{tabular}{|c|c|c|c|c|c|c|}
\hline $\mathrm{f}$ & $\begin{array}{c}\mathrm{BP} \\
{\left[\mathrm{g} / \mathrm{cm}^{2}\right]}\end{array}$ & $\begin{array}{l}\langle\lambda\rangle_{p, c a l} \\
{\left[\mathrm{~g} / \mathrm{cm}^{2}\right]}\end{array}$ & $\begin{array}{l}\langle\lambda\rangle_{F e, c a l} \\
{\left[\mathrm{~g} / \mathrm{cm}^{2}\right]}\end{array}$ & $\mathrm{f}_{B P}$ & $\begin{array}{c}\langle\lambda\rangle_{p, \text { sim }}-\langle\lambda\rangle_{p, c a l} \\
{\left[\mathrm{~g} / \mathrm{cm}^{2}\right]}\end{array}$ & $\begin{array}{c}\langle\lambda\rangle_{F e, \text { sim }}-\langle\lambda\rangle_{F e, \text { cal }} \\
{\left[\mathrm{g} / \mathrm{cm}^{2}\right]}\end{array}$ \\
\hline 0.9 & 76.03 & 35.8 & 15.06 & 0.12 & -0.40 & -0.01 \\
\hline 0.8 & 76.03 & 35.84 & 15.32 & 0.11 & -0.44 & -0.27 \\
\hline 0.7 & 76.03 & 36.57 & 14.68 & 0.09 & -1.17 & 0.37 \\
\hline 0.6 & 76.03 & 36.54 & 13.66 & 0.08 & -1.14 & 1.39 \\
\hline 0.5 & 76.03 & 37.33 & 13.02 & 0.07 & -1.93 & 2.03 \\
\hline 0.4 & 76.03 & 38.26 & 12.42 & 0.05 & -2.86 & 2.63 \\
\hline 0.3 & 76.03 & 39.49 & 12.03 & 0.04 & -4.09 & 3.02 \\
\hline 0.2 & 76.03 & 40.74 & 11.55 & 0.03 & -5.34 & 3.50 \\
\hline 0.1 & 76.03 & 42.11 & 10.61 & 0.01 & -6.71 & 4.44 \\
\hline 0.9 & 70.76 & 35.66 & 13.95 & 0.15 & -0.26 & 1.10 \\
\hline 0.8 & 70.76 & 35.96 & 14.17 & 0.13 & -0.56 & 0.88 \\
\hline 0.7 & 70.76 & 36.25 & 14.11 & 0.11 & -0.85 & 0.94 \\
\hline 0.6 & 70.76 & 36.63 & 13.48 & 0.1 & -1.23 & 1.57 \\
\hline 0.5 & 70.76 & 37.54 & 12.67 & 0.08 & -2.14 & 2.38 \\
\hline 0.4 & 70.76 & 38.29 & 12.05 & 0.07 & -2.89 & 3.00 \\
\hline 0.3 & 70.76 & 39.85 & 11.46 & 0.05 & -4.45 & 3.59 \\
\hline 0.2 & 70.76 & 41.07 & 11.13 & 0.03 & -5.67 & 3.92 \\
\hline 0.1 & 70.76 & 41.53 & 10.4 & 0.02 & -6.13 & 4.65 \\
\hline 0.9 & 65.1 & 35.3 & 13.09 & 0.16 & 0.10 & 1.96 \\
\hline 0.8 & 65.1 & 35.54 & 13.65 & 0.14 & -0.14 & 1.40 \\
\hline 0.7 & 65.1 & 35.82 & 13.52 & 0.12 & -0.42 & 1.53 \\
\hline 0.6 & 65.1 & 36.06 & 12.89 & 0.11 & -0.66 & 2.16 \\
\hline 0.5 & 65.1 & 36.83 & 12.06 & 0.09 & -1.43 & 2.99 \\
\hline 0.4 & 65.1 & 37.54 & 11.17 & 0.07 & -2.14 & 3.88 \\
\hline 0.3 & 65.1 & 38.54 & 10.75 & 0.05 & -3.14 & 4.30 \\
\hline 0.2 & 65.1 & 40.04 & 10.4 & 0.04 & -4.64 & 4.65 \\
\hline 0.1 & 65.1 & 41.29 & 9.76 & 0.02 & -5.89 & 5.29 \\
\hline 0.9 & 58.88 & 36.01 & 13.11 & 0.2 & -0.61 & 1.94 \\
\hline 0.8 & 58.88 & 36.02 & 13.24 & 0.18 & -0.62 & 1.81 \\
\hline 0.7 & 58.88 & 36.13 & 13.43 & 0.16 & -0.73 & 1.62 \\
\hline 0.6 & 58.88 & 36.34 & 12.77 & 0.14 & -0.94 & 2.28 \\
\hline 0.5 & 58.88 & 36.55 & 11.67 & 0.11 & -1.15 & 3.38 \\
\hline 0.4 & 58.88 & 37.08 & 10.7 & 0.09 & -1.68 & 4.35 \\
\hline 0.3 & 58.88 & 37.97 & 10.08 & 0.07 & -2.57 & 4.97 \\
\hline 0.2 & 58.88 & 39.34 & 9.7 & 0.05 & -3.94 & 5.35 \\
\hline 0.1 & 58.88 & 40.8 & 9.16 & 0.03 & -5.40 & 5.89 \\
\hline
\end{tabular}


Table 7.5: Results of different breaking points for various fractions of proton iron mixture. Mean free path values of proton and iron were computed with Monte Carlo Number Generator fed by SIBYLL values. Differences were calculated using the simulation values

\begin{tabular}{|c|c|c|c|c|c|c|}
\hline $\mathrm{f}$ & $\begin{array}{c}\mathrm{BP} \\
{\left[\mathrm{g} / \mathrm{cm}^{2}\right]}\end{array}$ & $\begin{array}{l}\langle\lambda\rangle_{p, c a l} \\
{\left[\mathrm{~g} / \mathrm{cm}^{2}\right]}\end{array}$ & $\begin{array}{l}\langle\lambda\rangle_{F e, c a l} \\
{\left[\mathrm{~g} / \mathrm{cm}^{2}\right]}\end{array}$ & $\mathrm{f}_{B P}$ & $\begin{array}{c}\langle\lambda\rangle_{p, \text { sim }}-\langle\lambda\rangle_{p, c a l} \\
{\left[\mathrm{~g} / \mathrm{cm}^{2}\right]}\end{array}$ & $\begin{array}{c}\langle\lambda\rangle_{F e, s i m}-\langle\lambda\rangle_{F e, c a l} \\
{\left[\mathrm{~g} / \mathrm{cm}^{2}\right]}\end{array}$ \\
\hline 0.90 & 76.03 & 35.39 & 15.28 & 0.10 & 0.01 & -6.00 \\
\hline 0.80 & 76.03 & 35.50 & 15.12 & 0.09 & -0.10 & -5.84 \\
\hline 0.70 & 76.03 & 35.85 & 14.82 & 0.08 & -0.45 & -5.54 \\
\hline 0.60 & 76.03 & 36.09 & 14.32 & 0.07 & -0.69 & -5.04 \\
\hline$\overline{0.50}$ & 76.03 & 36.99 & 13.53 & 0.06 & -1.59 & -4.25 \\
\hline 0.40 & 76.03 & 37.31 & 12.63 & 0.04 & -1.91 & -3.35 \\
\hline 0.30 & 76.03 & 38.75 & 11.85 & 0.03 & -3.35 & -2.57 \\
\hline 0.20 & 76.03 & 40.37 & 11.20 & 0.02 & -4.97 & -1.92 \\
\hline 0.10 & 76.03 & 41.64 & 10.06 & 0.01 & -6.24 & -0.78 \\
\hline$\overline{0.90}$ & 70.76 & 33.89 & 15.47 & 0.12 & 1.51 & -6.19 \\
\hline 0.80 & 70.76 & 34.10 & 15.46 & 0.11 & 1.30 & -6.18 \\
\hline 0.70 & 70.76 & 34.90 & 14.82 & 0.09 & 0.50 & -5.54 \\
\hline$\overline{0.60}$ & 70.76 & 34.88 & 14.03 & 0.08 & 0.52 & -4.75 \\
\hline 0.50 & 70.76 & 35.34 & 12.81 & 0.07 & 0.06 & -3.53 \\
\hline 0.40 & 70.76 & 36.20 & 11.84 & 0.05 & -0.80 & -2.56 \\
\hline 0.30 & 70.76 & 37.85 & 10.98 & 0.04 & -2.45 & -1.70 \\
\hline 0.20 & 70.76 & 40.33 & 10.59 & 0.03 & -4.93 & -1.31 \\
\hline 0.10 & 70.76 & 42.28 & 9.72 & 0.01 & -6.88 & -0.44 \\
\hline 0.90 & 65.10 & 35.78 & 13.51 & 0.13 & -0.38 & -4.23 \\
\hline 0.80 & 65.10 & 35.86 & 13.54 & 0.12 & -0.46 & -4.26 \\
\hline 0.70 & 65.10 & 36.11 & 13.47 & 0.10 & -0.71 & -4.19 \\
\hline 0.60 & 65.10 & 36.34 & 13.03 & 0.09 & -0.94 & -3.75 \\
\hline 0.50 & 65.10 & 37.10 & 12.37 & 0.07 & -1.70 & -3.09 \\
\hline 0.40 & 65.10 & 37.98 & 11.67 & 0.06 & -2.58 & -2.39 \\
\hline 0.30 & 65.10 & 39.42 & 11.21 & 0.04 & -4.02 & -1.93 \\
\hline 0.20 & 65.10 & 41.58 & 10.83 & 0.03 & -6.18 & -1.55 \\
\hline 0.10 & 65.10 & 42.53 & 9.85 & 0.01 & -7.13 & -0.57 \\
\hline 0.90 & 58.88 & 34.07 & 13.69 & 0.17 & 1.33 & -4.41 \\
\hline 0.80 & 58.88 & 34.07 & 13.41 & 0.15 & 1.33 & -4.13 \\
\hline 0.70 & 58.88 & 34.33 & 12.99 & 0.13 & 1.07 & -3.71 \\
\hline 0.60 & 58.88 & 34.64 & 12.51 & 0.12 & 0.76 & -3.23 \\
\hline 0.50 & 58.88 & 35.09 & 11.12 & 0.10 & 0.31 & -1.84 \\
\hline 0.40 & 58.88 & 36.11 & 10.67 & 0.08 & -0.71 & -1.39 \\
\hline 0.30 & 58.88 & 37.57 & 10.11 & 0.06 & -2.17 & -0.83 \\
\hline 0.20 & 58.88 & 39.74 & 9.82 & 0.04 & -4.34 & -0.54 \\
\hline 0.10 & 58.88 & 41.63 & 9.21 & 0.02 & -6.23 & 0.07 \\
\hline
\end{tabular}


Table 7.6: Results of different breaking points for various fractions of proton iron mixture. Mean free path values of proton and iron were computed with Monte Carlo Number Generator fed by EPOS values. Differences were calculated using the simulation values

\begin{tabular}{|c|c|c|c|c|c|c|}
\hline $\mathrm{f}$ & $\begin{array}{c}\mathrm{BP} \\
{\left[\mathrm{g} / \mathrm{cm}^{2}\right]}\end{array}$ & $\begin{array}{l}\langle\lambda\rangle_{p, c a l} \\
{\left[\mathrm{~g} / \mathrm{cm}^{2}\right]}\end{array}$ & $\begin{array}{l}\langle\lambda\rangle_{F e, c a l} \\
{\left[\mathrm{~g} / \mathrm{cm}^{2}\right]}\end{array}$ & $\mathrm{f}_{B P}$ & $\begin{array}{c}\langle\lambda\rangle_{p, \text { sim }}-\langle\lambda\rangle_{p, c a l} \\
{\left[\mathrm{~g} / \mathrm{cm}^{2}\right]}\end{array}$ & $\begin{array}{c}\langle\lambda\rangle_{F e, s i m}-\langle\lambda\rangle_{F e, c a l} \\
{\left[\mathrm{~g} / \mathrm{cm}^{2}\right]}\end{array}$ \\
\hline 0.90 & 76.03 & 33.78 & 16.01 & 0.12 & 5.56 & -5.96 \\
\hline 0.80 & 76.03 & 34.01 & 15.94 & 0.10 & 5.33 & -5.89 \\
\hline 0.70 & 76.03 & 34.64 & 15.59 & 0.09 & 4.70 & -5.54 \\
\hline 0.60 & 76.03 & 35.07 & 14.58 & 0.08 & 4.27 & -4.53 \\
\hline$\overline{0.50}$ & 76.03 & 35.75 & 13.49 & 0.06 & 3.59 & -3.44 \\
\hline 0.40 & 76.03 & 36.84 & 12.07 & 0.05 & 2.50 & -2.02 \\
\hline 0.30 & 76.03 & 38.18 & 11.35 & 0.04 & 1.16 & -1.30 \\
\hline 0.20 & 76.03 & 40.18 & 10.92 & 0.03 & -0.84 & -0.87 \\
\hline 0.10 & 76.03 & 41.64 & 10.20 & 0.01 & -2.30 & -0.15 \\
\hline$\overline{0.90}$ & 70.76 & 35.66 & 13.56 & 0.14 & 3.68 & -3.51 \\
\hline 0.80 & 70.76 & 36.03 & 14.02 & 0.12 & 3.31 & -3.97 \\
\hline 0.70 & 70.76 & 36.25 & 14.20 & 0.11 & 3.09 & -4.15 \\
\hline 0.60 & 70.76 & 36.89 & 13.72 & 0.09 & 2.45 & -3.67 \\
\hline 0.50 & 70.76 & 37.37 & 12.93 & 0.08 & 1.97 & -2.88 \\
\hline 0.40 & 70.76 & 38.53 & 12.16 & 0.06 & 0.81 & -2.11 \\
\hline 0.30 & 70.76 & 39.72 & 11.73 & 0.05 & -0.38 & -1.68 \\
\hline 0.20 & 70.76 & 41.21 & 11.24 & 0.03 & -1.87 & -1.19 \\
\hline 0.10 & 70.76 & 42.34 & 10.39 & 0.02 & -3.00 & -0.34 \\
\hline 0.90 & 65.10 & 34.09 & 13.81 & 0.15 & 5.25 & -3.76 \\
\hline 0.80 & 65.10 & 34.31 & 14.30 & 0.14 & 5.03 & -4.25 \\
\hline 0.70 & 65.10 & 34.62 & 14.49 & 0.12 & 4.72 & -4.44 \\
\hline 0.60 & 65.10 & 34.94 & 13.52 & 0.10 & 4.40 & -3.47 \\
\hline 0.50 & 65.10 & 35.65 & 12.22 & 0.09 & 3.69 & -2.17 \\
\hline 0.40 & 65.10 & 36.68 & 11.14 & 0.07 & 2.66 & -1.09 \\
\hline 0.30 & 65.10 & 37.79 & 10.55 & 0.05 & 1.55 & -0.50 \\
\hline 0.20 & 65.10 & 39.08 & 10.16 & 0.04 & 0.26 & -0.11 \\
\hline 0.10 & 65.10 & 41.31 & 9.54 & 0.02 & -1.97 & 0.51 \\
\hline 0.90 & 58.88 & 34.22 & 12.71 & 0.20 & 5.12 & -2.66 \\
\hline 0.80 & 58.88 & 34.32 & 13.73 & 0.18 & 5.02 & -3.68 \\
\hline 0.70 & 58.88 & 34.59 & 13.60 & 0.15 & 4.75 & -3.55 \\
\hline 0.60 & 58.88 & 34.91 & 12.84 & 0.13 & 4.43 & -2.79 \\
\hline 0.50 & 58.88 & 35.33 & 11.55 & 0.11 & 4.01 & -1.50 \\
\hline 0.40 & 58.88 & 36.22 & 10.74 & 0.09 & 3.12 & -0.69 \\
\hline 0.30 & 58.88 & 37.55 & 10.09 & 0.07 & 1.79 & -0.04 \\
\hline 0.20 & 58.88 & 39.35 & 9.81 & 0.05 & -0.01 & 0.24 \\
\hline 0.10 & 58.88 & 41.09 & 9.27 & 0.03 & -1.75 & 0.78 \\
\hline
\end{tabular}




\subsubsection{Testing CNO-Fe composition}

As was done for the other mixtures, a similar analysis was carried out for CNO iron mixture. BP's corresponding to different $\gamma$ and $\beta$ values were tested for different mixture ratios of $\mathrm{CNO}$ and $\mathrm{Fe}$.

The discrepancies between the simulation values and calculated values via BP's for CNO were shown in Figs. 7.22(a), 7.23(a), 7.24(a) in color scale. Darker colors show lower discrepancies. For iron, discrepancies were shown in Figs. 7.22(b), 7.22(b), 7.22(b). Colors close to background color correspond to values close to zero. The best choice of break

point would be between $30-40 \mathrm{~g} / \mathrm{cm}^{2}$. Detailed tables for the break points falling into the range were tabulated in Tables 7.7, 7.8 and 7.9. To minimize the error for all three models, $31.91 \mathrm{~g} / \mathrm{cm}^{2}$ was selected as the break point for proton and iron mixture. Similar to the $\mathrm{p}$ $\mathrm{CNO}$ and $\mathrm{p}-\mathrm{Fe}$ composition discussions, the critical fraction below which one will assume another composition was found to be $10 \%$.

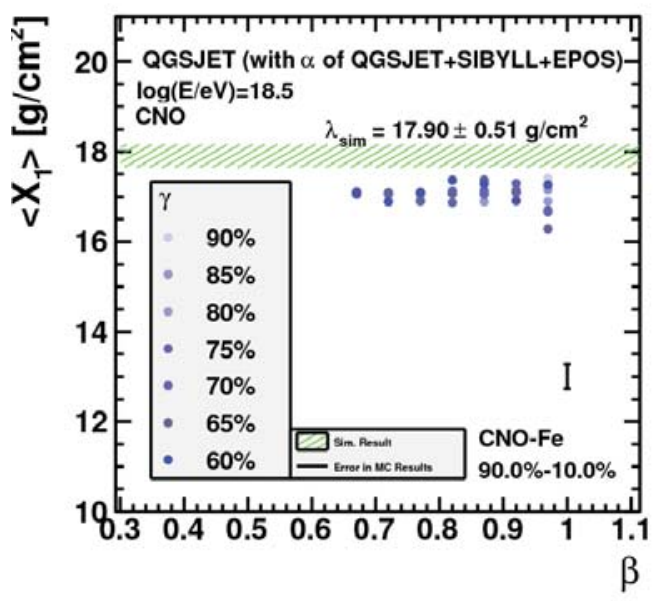

(a)

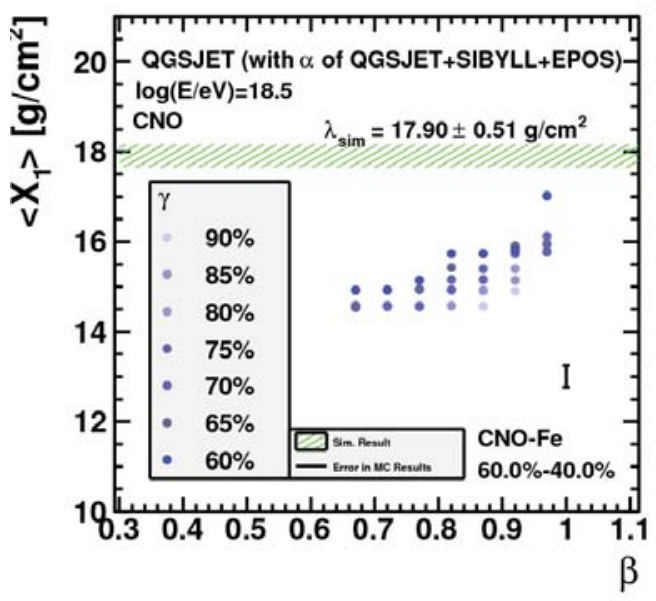

(b)

Figure 7.19: Results of bootstrap analysis for different $\beta$ and $\gamma$ values applied to QGSJET simulations. Results for proton first interaction length are shown in (a) for $90 \% \mathrm{CNO}-10 \%$ iron mixture and (b) for $60 \% \mathrm{CNO}-40 \%$ iron mixture. 


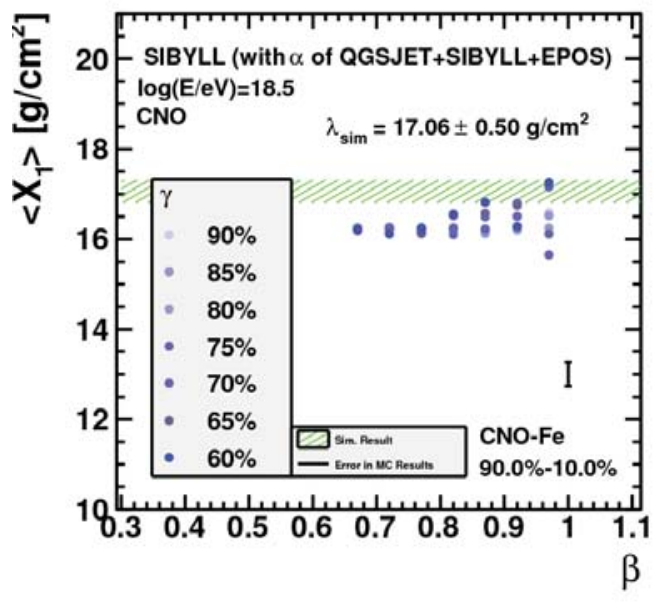

(a)

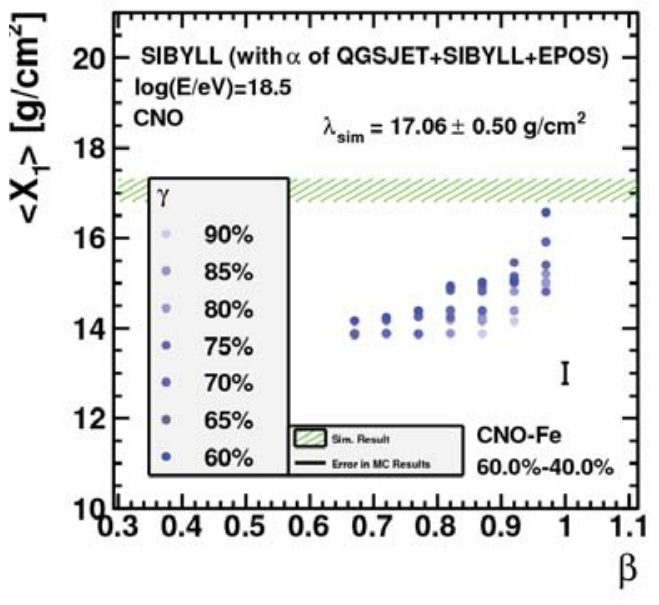

(b)

Figure 7.20: Results of bootstrap analysis for different $\beta$ and $\gamma$ values applied to SIBYLL simulations. Results for proton first interaction length are shown in (a) for $90 \% \mathrm{CNO}-10 \%$ iron mixture and (b) for $60 \%$ CNO - $40 \%$ iron mixture.

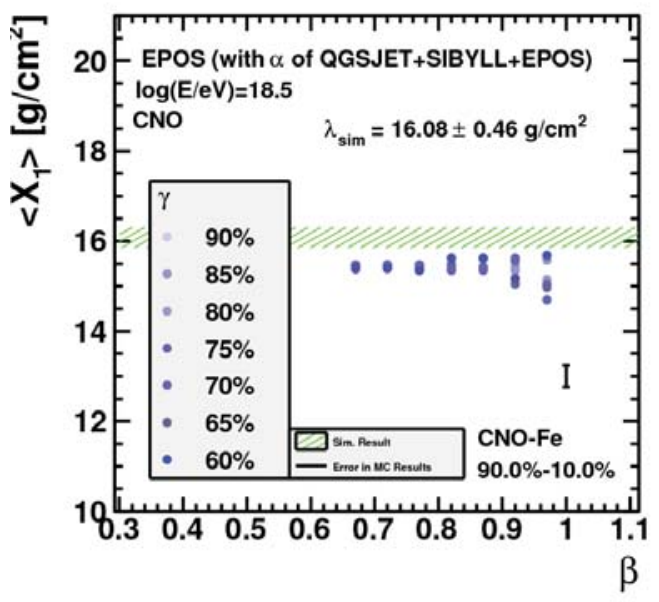

(a)

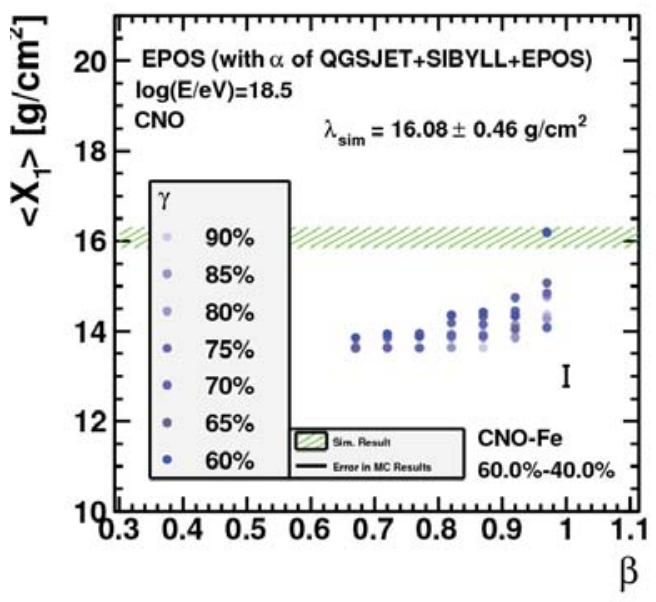

(b)

Figure 7.21: Results of bootstrap analysis for different $\beta$ and $\gamma$ values applied to EPOS simulations. Results for proton first interaction length are shown in (a) for $90 \% \mathrm{CNO}-10 \%$ iron mixture and (b) for $60 \% \mathrm{CNO}-40 \%$ iron mixture. 


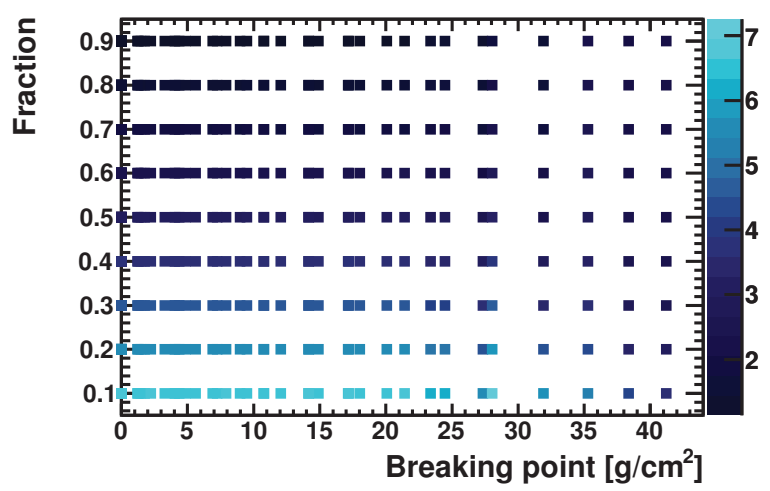

(a)

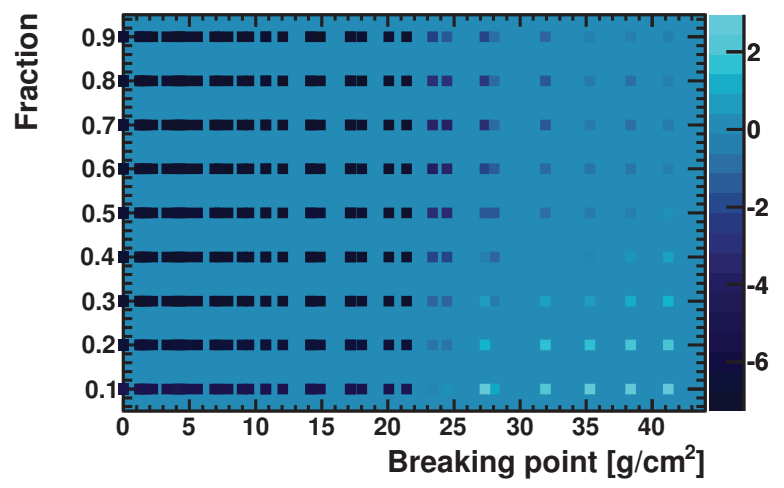

(b)

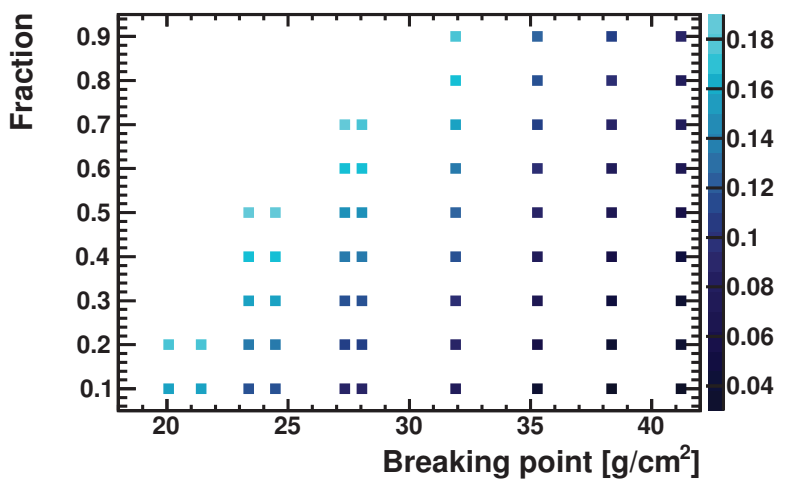

(c)

Figure 7.22: Results of different break points for different $\mathrm{CNO}$ and iron mixtures using QGSJET interaction model values. $\mathrm{y}$-axis is the fraction of $\mathrm{CNO}$ in the mixture. Color codes indicate the difference of calculated mean free path and the simulation value (a) for CNO and (b) for iron, and (c) fraction of data after the break point for the first interaction length distributions. Data points out of the range are not plotted. 


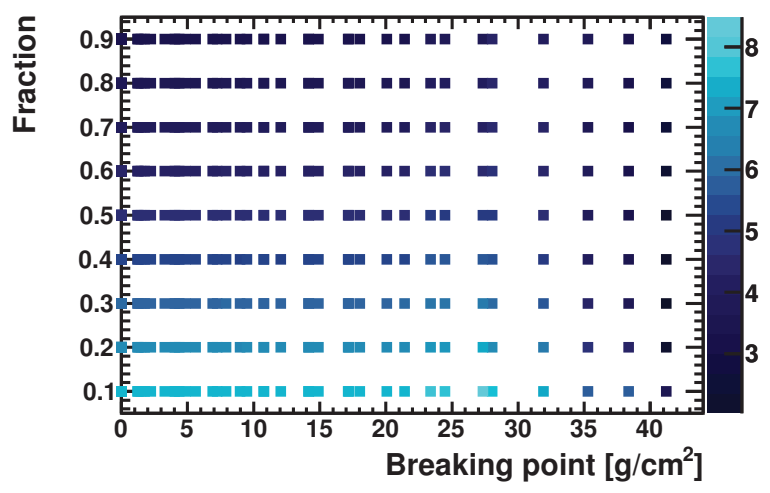

(a)

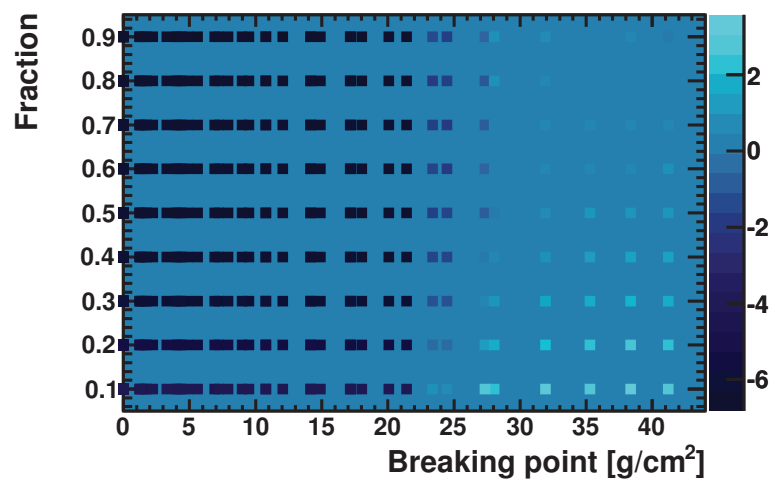

(b)

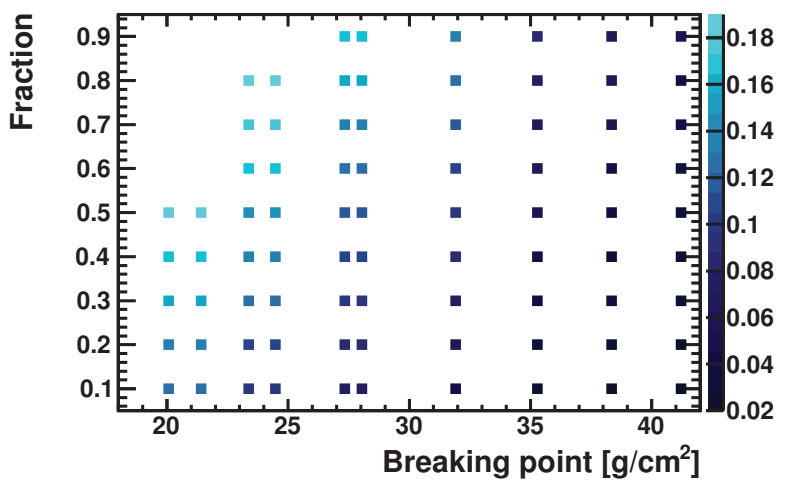

(c)

Figure 7.23: Results of different break points for different CNO and iron mixtures using SIBYLL interaction model values. $\mathrm{y}$-axis is the fraction of $\mathrm{CNO}$ in the mixture. Color codes indicate the difference of calculated mean free path and the simulation value (a) for CNO and (b) for iron, and (c) fraction of data after the break point for the first interaction length distributions. Data points out of the range are not plotted. 


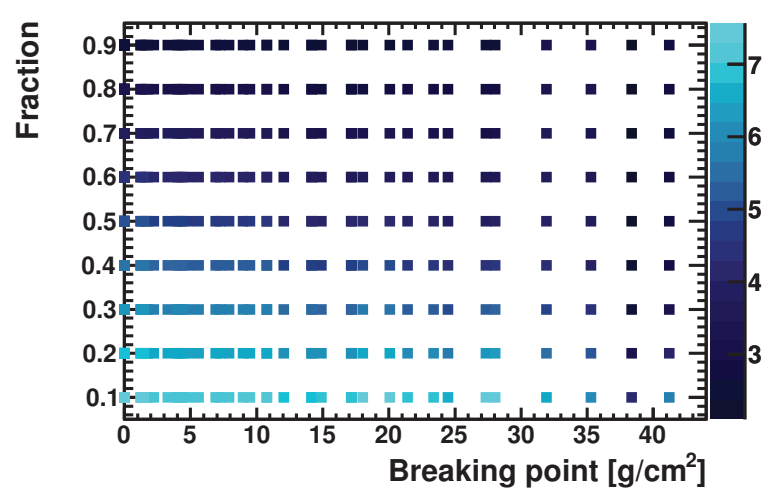

(a)

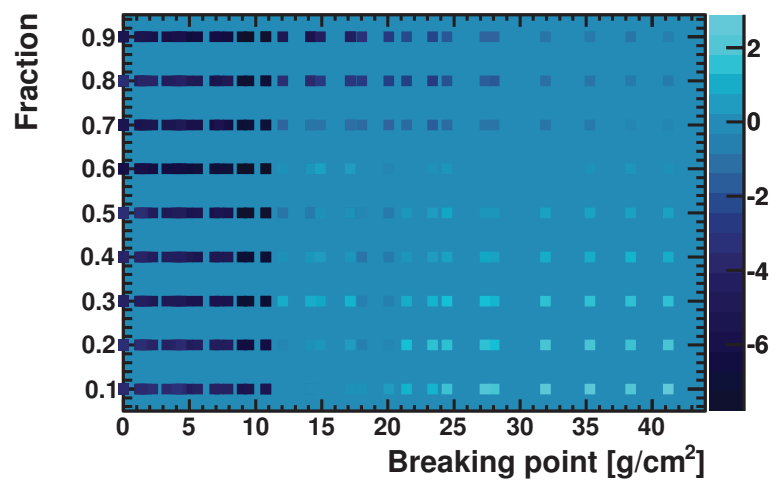

(b)

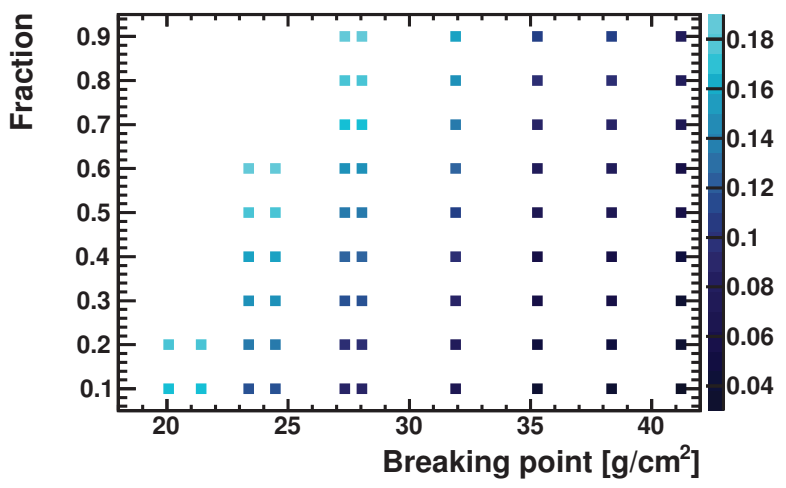

(c)

Figure 7.24: Results of different break points for different $\mathrm{CNO}$ and iron mixtures using EPOS interaction model values. $\mathrm{y}$-axis is the fraction of $\mathrm{CNO}$ in the mixture. Color codes indicate the difference of calculated mean free path and the simulation value (a) for CNO and (b) for iron, and (c) fraction of data after the break point for the first interaction length distributions. Data points out of the range are not plotted. 
Table 7.7: Results of different breaking points for various fractions of CNO iron mixture. Mean free path values of $\mathrm{CNO}$ and iron were computed with Monte Carlo Number Generator fed by QGSJET values. Differences were calculated using the simulation values

\begin{tabular}{|c|c|c|c|c|c|c|}
\hline f & $\begin{array}{c}\mathrm{BP} \\
{\left[\mathrm{g} / \mathrm{cm}^{2}\right]}\end{array}$ & $\begin{array}{c}\langle\lambda\rangle_{\text {CNO,cal }} \\
{\left[\mathrm{g} / \mathrm{cm}^{2}\right]}\end{array}$ & $\begin{array}{l}\langle\lambda\rangle_{F e, c a l} \\
{\left[\mathrm{~g} / \mathrm{cm}^{2}\right]}\end{array}$ & $\mathrm{f}_{B P}$ & $\begin{array}{c}\langle\lambda\rangle_{C N O, \text { sim }}-\langle\lambda\rangle_{C N O, c a l} \\
{\left[\mathrm{~g} / \mathrm{cm}^{2}\right]}\end{array}$ & $\begin{array}{c}\langle\lambda\rangle_{F e, \text { sim }}-\langle\lambda\rangle_{F e, c a l} \\
{\left[\mathrm{~g} / \mathrm{cm}^{2}\right]}\end{array}$ \\
\hline 0.90 & 38.35 & 15.52 & 10.09 & 0.11 & 2.25 & 0.17 \\
\hline 0.80 & 38.35 & 15.44 & 10.58 & 0.10 & 2.33 & -0.32 \\
\hline 0.70 & 38.35 & 15.48 & 10.55 & 0.09 & 2.29 & -0.29 \\
\hline 0.60 & 38.35 & 15.34 & 10.35 & 0.08 & 2.43 & -0.09 \\
\hline 0.50 & 38.35 & 15.24 & 10.12 & 0.07 & 2.53 & 0.14 \\
\hline 0.40 & 38.35 & 15.06 & 9.28 & 0.06 & 2.71 & 0.98 \\
\hline 0.30 & 38.35 & 14.76 & 8.58 & 0.05 & 3.01 & 1.68 \\
\hline 0.20 & 38.35 & 14.21 & 7.77 & 0.04 & 3.56 & 2.49 \\
\hline 0.10 & 38.35 & 13.40 & 7.11 & 0.03 & 4.37 & 3.15 \\
\hline 0.90 & 35.28 & 15.33 & 9.99 & 0.13 & 2.44 & 0.27 \\
\hline 0.80 & 35.28 & 15.23 & 10.26 & 0.12 & 2.54 & 0.00 \\
\hline 0.70 & 35.28 & 15.12 & 10.32 & 0.11 & 2.65 & -0.06 \\
\hline 0.60 & 35.28 & 14.91 & 10.50 & 0.10 & 2.86 & -0.24 \\
\hline 0.50 & 35.28 & 14.65 & 10.34 & 0.09 & 3.12 & -0.08 \\
\hline 0.40 & 35.28 & 14.34 & 9.87 & 0.08 & 3.43 & 0.39 \\
\hline 0.30 & 35.28 & 13.86 & 9.07 & 0.07 & 3.91 & 1.19 \\
\hline 0.20 & 35.28 & 13.20 & 8.00 & 0.06 & 4.57 & 2.26 \\
\hline 0.10 & 35.28 & 12.21 & 7.13 & 0.04 & 5.56 & 3.13 \\
\hline 0.90 & 31.91 & 16.13 & 11.07 & 0.18 & 1.64 & -0.81 \\
\hline 0.80 & 31.91 & 15.97 & 11.30 & 0.17 & 1.80 & -1.04 \\
\hline 0.70 & 31.91 & 15.76 & 11.35 & 0.16 & 2.01 & -1.09 \\
\hline 0.60 & 31.91 & 15.46 & 10.84 & 0.14 & 2.31 & -0.58 \\
\hline 0.50 & 31.91 & 15.16 & 10.66 & 0.13 & 2.61 & -0.40 \\
\hline 0.40 & 31.91 & 14.72 & 9.73 & 0.12 & 3.05 & 0.53 \\
\hline 0.30 & 31.91 & 14.07 & 8.91 & 0.10 & 3.70 & 1.35 \\
\hline 0.20 & 31.91 & 13.18 & 8.04 & 0.09 & 4.59 & 2.22 \\
\hline 0.10 & 31.91 & 11.94 & 7.44 & 0.08 & 5.83 & 2.82 \\
\hline 0.90 & 28.05 & 15.71 & 10.59 & 0.22 & 2.06 & -0.33 \\
\hline$\overline{0.80}$ & 28.05 & 15.46 & 10.80 & 0.20 & 2.31 & -0.54 \\
\hline 0.70 & 28.05 & 15.16 & 10.82 & 0.18 & 2.61 & -0.56 \\
\hline 0.60 & 28.05 & 14.78 & 11.19 & 0.17 & 2.99 & -0.93 \\
\hline 0.50 & 28.05 & 14.32 & 11.41 & 0.15 & 3.45 & -1.15 \\
\hline 0.40 & 28.05 & 13.68 & 10.92 & 0.14 & 4.09 & -0.66 \\
\hline 0.30 & 28.05 & 12.91 & 10.22 & 0.12 & 4.86 & 0.04 \\
\hline 0.20 & 28.05 & 11.86 & 9.79 & 0.11 & 5.91 & 0.47 \\
\hline 0.10 & 28.05 & 10.46 & 8.79 & 0.09 & 7.31 & 1.47 \\
\hline
\end{tabular}


Table 7.8: Results of different breaking points for various fractions of CNO iron mixture. Mean free path values of $\mathrm{CNO}$ and iron were computed with Monte Carlo Number Generator fed by SIBYLL values. Differences were calculated using the simulation values

\begin{tabular}{|c|c|c|c|c|c|c|}
\hline f & $\begin{array}{c}\mathrm{BP} \\
{\left[\mathrm{g} / \mathrm{cm}^{2}\right]}\end{array}$ & $\begin{array}{c}\langle\lambda\rangle_{\text {CNO,cal }} \\
{\left[\mathrm{g} / \mathrm{cm}^{2}\right]}\end{array}$ & $\begin{array}{l}\langle\lambda\rangle_{F e, c a l} \\
{\left[\mathrm{~g} / \mathrm{cm}^{2}\right]}\end{array}$ & $\mathrm{f}_{B P}$ & $\begin{array}{c}\langle\lambda\rangle_{C N O, \text { sim }}-\langle\lambda\rangle_{C N O, c a l} \\
{\left[\mathrm{~g} / \mathrm{cm}^{2}\right]}\end{array}$ & $\begin{array}{c}\langle\lambda\rangle_{F e, \text { sim }}-\langle\lambda\rangle_{F e, c a l} \\
{\left[\mathrm{~g} / \mathrm{cm}^{2}\right]}\end{array}$ \\
\hline 0.90 & 38.35 & 14.24 & 9.28 & 0.07 & 0.81 & 0.00 \\
\hline 0.80 & 38.35 & 14.22 & 9.43 & 0.07 & 0.83 & -0.15 \\
\hline 0.70 & 38.35 & 14.32 & 9.35 & 0.06 & 0.73 & -0.07 \\
\hline 0.60 & 38.35 & 14.29 & 9.27 & 0.05 & 0.76 & 0.01 \\
\hline 0.50 & 38.35 & 14.29 & 8.72 & 0.05 & 0.76 & 0.56 \\
\hline 0.40 & 38.35 & 14.16 & 8.42 & 0.04 & 0.89 & 0.86 \\
\hline 0.30 & 38.35 & 13.87 & 7.76 & 0.04 & 1.18 & 1.52 \\
\hline 0.20 & 38.35 & 13.24 & 7.02 & 0.03 & 1.81 & 2.26 \\
\hline 0.10 & 38.35 & 11.80 & 6.44 & 0.02 & 3.25 & 2.84 \\
\hline 0.90 & 35.28 & 13.82 & 9.42 & 0.09 & 1.23 & -0.14 \\
\hline 0.80 & 35.28 & 13.81 & 9.53 & 0.08 & 1.24 & -0.25 \\
\hline 0.70 & 35.28 & 13.85 & 9.41 & 0.07 & 1.20 & -0.13 \\
\hline 0.60 & 35.28 & 13.70 & 9.36 & 0.07 & 1.35 & -0.08 \\
\hline 0.50 & 35.28 & 13.66 & 8.82 & 0.06 & 1.39 & 0.46 \\
\hline 0.40 & 35.28 & 13.55 & 8.59 & 0.05 & 1.50 & 0.69 \\
\hline 0.30 & 35.28 & 13.25 & 8.02 & 0.05 & 1.80 & 1.26 \\
\hline 0.20 & 35.28 & 12.73 & 7.44 & 0.04 & 2.32 & 1.84 \\
\hline 0.10 & 35.28 & 11.79 & 6.85 & 0.03 & 3.26 & 2.43 \\
\hline 0.90 & 31.91 & 13.53 & 9.10 & 0.14 & 1.52 & 0.18 \\
\hline 0.80 & 31.91 & 13.42 & 9.38 & 0.13 & 1.63 & -0.10 \\
\hline 0.70 & 31.91 & 13.26 & 9.35 & 0.12 & 1.79 & -0.07 \\
\hline 0.60 & 31.91 & 13.10 & 9.25 & 0.11 & 1.95 & 0.03 \\
\hline 0.50 & 31.91 & 12.86 & 9.05 & 0.10 & 2.19 & 0.23 \\
\hline 0.40 & 31.91 & 12.51 & 8.86 & 0.09 & 2.54 & 0.42 \\
\hline 0.30 & 31.91 & 11.97 & 8.27 & 0.08 & 3.08 & 1.01 \\
\hline 0.20 & 31.91 & 11.25 & 7.67 & 0.07 & 3.80 & 1.61 \\
\hline 0.10 & 31.91 & 10.35 & 6.70 & 0.05 & 4.70 & 2.58 \\
\hline 0.90 & 28.05 & 13.22 & 9.04 & 0.17 & 1.83 & 0.24 \\
\hline$\overline{0.80}$ & 28.05 & 13.10 & 8.99 & 0.16 & 1.95 & 0.29 \\
\hline 0.70 & 28.05 & 12.97 & 9.47 & 0.14 & 2.08 & -0.19 \\
\hline 0.60 & 28.05 & 12.71 & 9.58 & 0.13 & 2.34 & -0.30 \\
\hline 0.50 & 28.05 & 12.47 & 9.68 & 0.12 & 2.58 & -0.40 \\
\hline 0.40 & 28.05 & 12.10 & 9.30 & 0.11 & 2.95 & -0.02 \\
\hline 0.30 & 28.05 & 11.57 & 8.84 & 0.10 & 3.48 & 0.44 \\
\hline 0.20 & 28.05 & 10.86 & 8.01 & 0.09 & 4.19 & 1.27 \\
\hline 0.10 & 28.05 & 10.02 & 7.45 & 0.08 & 5.03 & 1.83 \\
\hline
\end{tabular}


Table 7.9: Results of different breaking points for various fractions of $\mathrm{CNO}$ iron mixture. Mean free path values of $\mathrm{CNO}$ and iron were computed with Monte Carlo Number Generator fed by EPOS values. Differences were calculated using the simulation values

\begin{tabular}{|c|c|c|c|c|c|c|}
\hline f & $\begin{array}{c}\mathrm{BP} \\
{\left[\mathrm{g} / \mathrm{cm}^{2}\right]}\end{array}$ & $\begin{array}{c}\langle\lambda\rangle_{C N O, \text { cal }} \\
{\left[\mathrm{g} / \mathrm{cm}^{2}\right]}\end{array}$ & $\begin{array}{l}\langle\lambda\rangle_{F e, c a l} \\
{\left[\mathrm{~g} / \mathrm{cm}^{2}\right]}\end{array}$ & $\mathrm{f}_{B P}$ & $\begin{array}{c}\langle\lambda\rangle_{C N O, \text { sim }}-\langle\lambda\rangle_{C N O, c a l} \\
{\left[\mathrm{~g} / \mathrm{cm}^{2}\right]}\end{array}$ & $\begin{array}{c}\langle\lambda\rangle_{F e, s i m}-\langle\lambda\rangle_{F e, c a l} \\
{\left[\mathrm{~g} / \mathrm{cm}^{2}\right]}\end{array}$ \\
\hline 0.90 & 38.35 & 15.54 & 10.70 & 0.11 & 0.63 & -0.65 \\
\hline 0.80 & 38.35 & 15.53 & 10.55 & 0.10 & 0.64 & -0.50 \\
\hline 0.70 & 38.35 & 15.61 & 10.16 & 0.09 & 0.56 & -0.11 \\
\hline 0.60 & 38.35 & 15.49 & 9.50 & 0.08 & 0.68 & 0.55 \\
\hline 0.50 & 38.35 & 15.46 & 9.11 & 0.07 & 0.71 & 0.94 \\
\hline 0.40 & 38.35 & 15.30 & 8.57 & 0.06 & 0.87 & 1.48 \\
\hline 0.30 & 38.35 & 15.04 & 8.19 & 0.06 & 1.13 & 1.86 \\
\hline 0.20 & 38.35 & 14.37 & 7.97 & 0.05 & 1.80 & 2.08 \\
\hline 0.10 & 38.35 & 13.36 & 7.64 & 0.04 & 2.81 & 2.41 \\
\hline 0.90 & 35.28 & 14.40 & 10.48 & 0.11 & 1.77 & -0.43 \\
\hline 0.80 & 35.28 & 14.33 & 10.71 & 0.10 & 1.84 & -0.66 \\
\hline 0.70 & 35.28 & 14.28 & 10.42 & 0.09 & 1.89 & -0.37 \\
\hline 0.60 & 35.28 & 14.06 & 9.76 & 0.08 & 2.11 & 0.29 \\
\hline 0.50 & 35.28 & 13.86 & 9.08 & 0.07 & 2.31 & 0.97 \\
\hline 0.40 & 35.28 & 13.61 & 8.69 & 0.06 & 2.56 & 1.36 \\
\hline 0.30 & 35.28 & 13.11 & 8.24 & 0.06 & 3.06 & 1.81 \\
\hline 0.20 & 35.28 & 12.45 & 7.88 & 0.05 & 3.72 & 2.17 \\
\hline 0.10 & 35.28 & 11.52 & 7.47 & 0.04 & 4.65 & 2.58 \\
\hline 0.90 & 31.91 & 14.42 & 10.60 & 0.16 & 1.75 & -0.55 \\
\hline 0.80 & 31.91 & 14.29 & 10.89 & 0.15 & 1.88 & -0.84 \\
\hline 0.70 & 31.91 & 14.11 & 10.57 & 0.14 & 2.06 & -0.52 \\
\hline 0.60 & 31.91 & 13.90 & 9.94 & 0.13 & 2.27 & 0.11 \\
\hline 0.50 & 31.91 & 13.62 & 9.13 & 0.11 & 2.55 & 0.92 \\
\hline 0.40 & 31.91 & 13.26 & 8.81 & 0.10 & 2.91 & 1.24 \\
\hline 0.30 & 31.91 & 12.72 & 8.17 & 0.09 & 3.45 & 1.88 \\
\hline 0.20 & 31.91 & 12.04 & 7.83 & 0.08 & 4.13 & 2.22 \\
\hline 0.10 & 31.91 & 11.09 & 7.44 & 0.07 & 5.08 & 2.61 \\
\hline 0.90 & 28.05 & 15.04 & 11.15 & 0.19 & 1.13 & -1.10 \\
\hline$\overline{0.80}$ & 28.05 & 14.78 & 11.65 & 0.18 & 1.39 & -1.60 \\
\hline 0.70 & 28.05 & 14.48 & 10.71 & 0.17 & 1.69 & -0.66 \\
\hline 0.60 & 28.05 & 14.08 & 9.96 & 0.15 & 2.09 & 0.09 \\
\hline 0.50 & 28.05 & 13.63 & 9.49 & 0.14 & 2.54 & 0.56 \\
\hline 0.40 & 28.05 & 13.03 & 9.18 & 0.13 & 3.14 & 0.87 \\
\hline 0.30 & 28.05 & 12.24 & 8.59 & 0.12 & 3.93 & 1.46 \\
\hline 0.20 & 28.05 & 11.31 & 8.34 & 0.10 & 4.86 & 1.71 \\
\hline 0.10 & 28.05 & 10.19 & 7.83 & 0.09 & 5.98 & 2.22 \\
\hline
\end{tabular}




\subsection{Choosing the proper break point}

The outcome of the method for individual compositions were described in previous sections. The selected break points for $\mathrm{p}-\mathrm{CNO}$ and $\mathrm{p}-\mathrm{Fe}$ were notably close to each other. Even though the mean free paths of $\mathrm{CNO}$ and iron were not the same, they were close enough for the analytical solution of Eq. Eqn 7.6 to yield relatively close results.

Other than the possible injection of cosmic rays at source sites, there are no clear indications of $\mathrm{p}-\mathrm{CNO}$ and $\mathrm{p}-\mathrm{Fe}$ transitions. It can be assumed at this stage that they are equally likely to be observed. Thus, upon the application of the BP's, in order to address the both possibilities at the same time, the close break points of $\mathrm{p}-\mathrm{CNO}$ and $\mathrm{p}-\mathrm{Fe}$ compositions needed to be reconsidered to simplify into a singular solution. Despite changing the original values retrieved through Eq. Eqn 7.6, the changes corresponded to insignificant changes in the $\gamma$ and $\beta$ values. The average of the two BP's were calculated and assigned for $\mathrm{p}-\mathrm{CNO}$ and $\mathrm{p}-\mathrm{Fe}$ mixtures.

To summarize, the following algorithm has been generated for examination of possible scenarios:

1: $\mathrm{X}_{B P}=70.44 \mathrm{~g} / \mathrm{cm}^{2}$ (assumes $\mathrm{p}-\mathrm{CNO}$ mix or $\mathrm{p}-\mathrm{Fe}$ )

2: if $\mathrm{f}_{\text {afterBP }} \leq 7 \%$ then

3: $\quad \mathrm{X}_{B P}=31.91 \mathrm{~g} / \mathrm{cm}^{2}$ (assumes CNO-Fe mix)

4: $\quad$ if $\mathrm{f}_{\text {afterBP }} \leq 10 \%$ then

5: $\quad \mathrm{X}_{B P}=15.00 \mathrm{~g} / \mathrm{cm}^{2}$ (assumes Fe only)

6: end if

7: end if

When the algorithm was applied to a given mixed composition, the information retrieved through the distribution after the BP was considered as the light particle information. Likewise, the information obtained by the distribution before the BP was considered as the combination of light and heavy particle information. In a p-CNO composition, proton was the light particle and $\mathrm{CNO}$ was the heavy particle. As for the $\mathrm{CNO}-\mathrm{Fe}$ mixture, $\mathrm{CNO}$ was the light particle and iron was the heavy particle in the mixture. 


\subsection{Calculating the Corrections due to BPM}

The results for the selected BP's were tabulated in Tables 7.1-7.9. Unidentified nature of the transition of the primary particles in cosmic rays hindered the use of a single value for correcting the bias as a consequence of the method. Thus. for the selected BP's, weighted averages of the biases for different hadronic interaction models and assumed primary mixtures were calculated. In Table 7.10, the averages of the biases for the light particle were given. The primaries written in bold were the light particles in the simulated compositions, and the calculated values correspond to their averages, respectively. Similarly, averages for the heavy particle were tabulated in Table 7.11.

Since the light particle in proton-CNO and proton-iron mixtures were proton, the bias of the light particle for this case should be considered together. Similarly, the heavy particle in proton-iron and CNO-iron mixtures were iron; thus they also need to be considered together. In addition, the heavy particle in proton-CNO mixture and the light particle in CNO-iron mixtures were CNO. Hence, they also should be considered together. In order to merge the information about these primaries in which they appear in multiple mixture scenarios, the same procedure of weighted averaging was applied for each primary particle. The calculated correction values for $\mathrm{p}, \mathrm{CNO}$ and iron are $1.37 \pm 0.61 \mathrm{~g} / \mathrm{cm}^{2}, 2.36 \pm 0.31$ $\mathrm{g} / \mathrm{cm}^{2}$ and $-0.29 \pm 0.45 \mathrm{~g} / \mathrm{cm}^{2}$, respectively. Upon the application of the BPM, the former correction would be applied.

Table 7.10: Weighted averages of biases of the light particle (printed in bold) for three different mixtures of primary particles $\left(\mathrm{m}_{p}<\mathrm{m}_{C N O}<\mathrm{m}_{F e}\right)$

\begin{tabular}{|c|c|c|c|}
\hline & primaries & Weighted Average & Error \\
\hline \multirow{3}{*}{ QGSJET } & $\mathbf{p}-\mathrm{CNO}$ & 5.47 & 1.47 \\
\cline { 2 - 4 } & $\mathbf{p}-\mathrm{Fe}$ & -2.38 & 1.63 \\
\cline { 2 - 4 } & $\mathbf{C N O}-\mathrm{Fe}$ & 2.85 & 0.61 \\
\hline \multirow{3}{*}{ EIBYLL } & $\mathbf{p}-\mathrm{CNO}$ & 0.45 & 1.37 \\
\cline { 2 - 4 } & $\mathbf{p}-\mathrm{Fe}$ & -0.47 & 1.45 \\
\cline { 2 - 4 } & $\mathbf{C N O}-\mathrm{Fe}$ & 2.26 & 0.64 \\
\hline \multirow{3}{*}{ General } & $\mathbf{p}-\mathrm{CNO}$ & 3.29 & 1.53 \\
\cline { 2 - 4 } & $\mathbf{p}-\mathrm{Fe}$ & 1.47 & 1.63 \\
\cline { 2 - 4 } & $\mathbf{C N O}-\mathrm{Pe}$ & 2.65 & 0.59 \\
\cline { 2 - 4 } & $\mathbf{p}-\mathrm{CNO}$ & 2.93 & 0.84 \\
\cline { 2 - 4 } & $\mathbf{p}-\mathrm{Fe}$ & -0.46 & 0.90 \\
\hline & $\mathbf{C N O}-\mathrm{Fe}$ & 2.60 & 0.35 \\
\hline
\end{tabular}


Table 7.11: Weighted averages of biases of the heavy particle (printed in bold) for three different mixtures of primary particles $\left(\mathrm{m}_{p}<\mathrm{m}_{C N O}<\mathrm{m}_{F e}\right)$

\begin{tabular}{|c|c|c|c|}
\hline & primaries & Weighted Average & Error \\
\hline \multirow{3}{*}{ QGSJET } & $\mathrm{p}-\mathbf{C N O}$ & 2.74 & 1.35 \\
\cline { 2 - 4 } & $\mathrm{p}-\mathbf{F e}$ & 2.73 & 1.13 \\
\cline { 2 - 4 } & $\mathrm{CNO}-\mathbf{F e}$ & 0.57 & 1.53 \\
\hline \multirow{3}{*}{ SIBYLL } & $\mathrm{p}-\mathbf{C N O}$ & 0.80 & 1.03 \\
\cline { 2 - 4 } & $\mathrm{p}-\mathbf{F e}$ & -2.39 & 0.90 \\
\cline { 2 - 4 } & $\mathrm{CNO}-\mathbf{F e}$ & 0.91 & 1.41 \\
\hline \multirow{3}{*}{ General } & $\mathrm{p}-\mathbf{C N O}$ & 1.56 & 1.11 \\
\cline { 2 - 4 } & $\mathrm{p}-\mathbf{F e}$ & -1.75 & 0.90 \\
\cline { 2 - 4 } & $\mathrm{CNO}-\mathbf{F e}$ & 1.03 & 1.14 \\
\hline & $\mathrm{p}-\mathbf{C N O}$ & 1.53 & 0.66 \\
\cline { 2 - 4 } & $\mathrm{p}-\mathbf{F e}$ & -0.91 & 0.56 \\
\cline { 2 - 4 } & $\mathrm{CNO}-\mathbf{F e}$ & 0.88 & 0.77 \\
\hline
\end{tabular}




\section{Chapter 8 Application of pmANN+BPM for 2 component simulated showers}

The prediction method based on Artificial Neural Networks (pmANN) was developed to predict the first interaction lengths of extensive air showers. Cumulatively, the distribution of the first interaction lengths of extensive air showers initiated by a certain cosmic ray particle yields its mean free path. The biases and errors associated with the assumed cosmic ray primaries were calculated. However, the possibility of cosmic rays being mixed at ultra high energies precluded using pmAnN by itself. Thus, following the pmAnN, the Break Point Method (BPM) was developed to elucidate the two primary mixed cosmic rays possibilities.

The application of the methods was carried out by successive application of pmAnN, bootstrap method and BPM. Using the CONEX shower simulation package, proton, $\mathrm{CNO}$ and iron extensive showers were generated for three hadronic interactions models (QGSJET, SIBYLL, EPOS). Then, showers were mixed at different ratios to mimic several assumed cosmic ray scenarios. For each composition scenario, a subset of showers were created using the bootstrap method. BPM was applied to each subset of showers to compute the mean values and errors for the corresponding mixture.

In order to test the efficiency of the combined model, a similar procedure was followed for the simulation values. Skipping pmANN, the simulation values were bootstrapped to create subsets of data. It should be noted that the subsets were the same subsets used for pmANN+BPM. Then, the subsets were run through BPM to calculate the mean values and errors of the primaries. This would also help to identify the possible problem of the joint method, if there were. 
Table 8.1: Correction values of different primaries for shower simulated with three hadronic interaction models at four energy bins

\begin{tabular}{|c|c|c|}
\hline primary & $\log (\mathrm{E} / \mathrm{eV})$ & Correction \\
\hline \multirow{4}{*}{ Proton } & 18.75 & $4.15 \pm 1.79$ \\
\cline { 2 - 3 } & 19.00 & $3.29 \pm 1.72$ \\
\cline { 2 - 3 } & 19.50 & $2.12 \pm 1.66$ \\
\cline { 2 - 3 } & 20.00 & $1.43 \pm 1.64$ \\
\hline \multirow{4}{*}{ CNO } & 18.75 & $4.07 \pm 0.69$ \\
\cline { 2 - 3 } & 19.00 & $3.75 \pm 0.67$ \\
\cline { 2 - 3 } & 19.50 & $3.04 \pm 0.66$ \\
\cline { 2 - 3 } & 20.00 & $3.41 \pm 0.64$ \\
\hline \multirow{4}{*}{ Iron } & 18.75 & $-0.19 \pm 0.86$ \\
\cline { 2 - 3 } & 19.00 & $-0.22 \pm 0.85$ \\
\cline { 2 - 3 } & 19.50 & $0.21 \pm 0.92$ \\
\cline { 2 - 3 } & 20.00 & $-0.13 \pm 0.92$ \\
\hline
\end{tabular}

The biases of the two methods, pmANN and BPM, were propagated for correction. A summary of the corrections are shown in Table 8.1. Even though the individual correction values were calculated, the application of the the individual values was problematic in practice. Since it is not certain whether it is a transition from proton to CNO or a the transition from proton to iron in reality, one cannot determine which correction value to use for the result of the pmANN+BPM. Thus, averages of the corrections weighted by the corresponding errors were calculated for proton-CNO and proton-iron composition assumption. The corrections were only applied to pmANN+BPM results. As for the simulation values, only BPM corrections would be applied. For the same reason, the correction values for proton-CNO and proton-iron compositions were reduced to a single correction value.

\subsection{Application of pmANN+BPM to QGSJET Showers}

The assumed compositions were tested by changing the light particle fraction in decrements of $10 \%$. pmANN+BPM was applied to QGSJET shower mixtures whereas only BPM was applied to first interaction length distributions of the simulations without corrections. Results of the combined method for QGSJET showers are shown in Figures 8.1-8.9) for proton-CNO, proton-iron and $\mathrm{CNO}$-iron mixtures. Assumed light particle fractions of the compositions are shown on $\mathrm{x}$-axis in figures. Differences of the calculated simulation mean free paths and pmANN+BPM estimations with expected values of the mean free paths 
were shown in Appendix A.1.

The break points were switched depending upon the fraction of data after a break point as explained in Section 7.4. The chosen value for the critical fraction was $7 \%$. This value corresponded to the fraction for chosen break point at 30\%(p)-70\%(CNO) mixture in the BPM analysis. Thus, when the results were checked, it was anticipated to notice the errors for proton mean free path change drastically when the fraction of proton in the mixture was below $30 \%$. The results demonstrates that the method fabricates a non-existing particle mean free path for low concentrations of proton in the composition. The sole reason for these predictions was that the second break point was devised for CNO-iron composition, not for proton-CNO composition. Same causalities could be seen for proton-iron compositions (Figures 8.1-8.3).

Break points were optimized to yield heavy particle mean free paths even at high concentration of light particle as described in Section 7.4. Despite this feature as was shown in the Monte-Carlo Simulations in Section 7.3, the same performance could not be obtained when the combined method was applied. It is evident that when the fraction of CNO is less than $30 \%$, the errors of the mean free path were high $\left(\sim 10 \mathrm{~g} / \mathrm{cm}^{2}\right)$.

The trends for the results obtained by the application of BPM to simulation values (fourth column in tables) were similar to the results predicted. Apart from the $30 \%$ limitation, errors were determined to be within the uncertainties of the predicted values. Taking into account the uncertainties in the predicted values, it was plausible to assess the combined method efficient for QGSJET showers.

The pmANN+BPM results at $\log (\mathrm{E} / \mathrm{eV})=19.50$ have exceptionally high uncertainties $(\sim 8$ $\mathrm{g} / \mathrm{cm}^{2}$ ) for proton-CNO and proton-iron mixtures. Similar characteristics were detected for $\log (\mathrm{E} / \mathrm{eV})=20.00$ as well. When the components of the uncertainties were investigated, it was found that the major contributor was the initial estimate before corrections applied.

The results for other composition scenario tested, CNO-Fe, shows no agreeable result at any energy bin. Comparing the simulation results (that use BPM only) and combined method results (that use pmANN+BPM), the source of the error can be associated with BPM since both sets of results at different energy bins exhibit similar characteristics.

The BP limitation for the CNO-Fe composition applies for cases with CNO less than $30 \%$. 


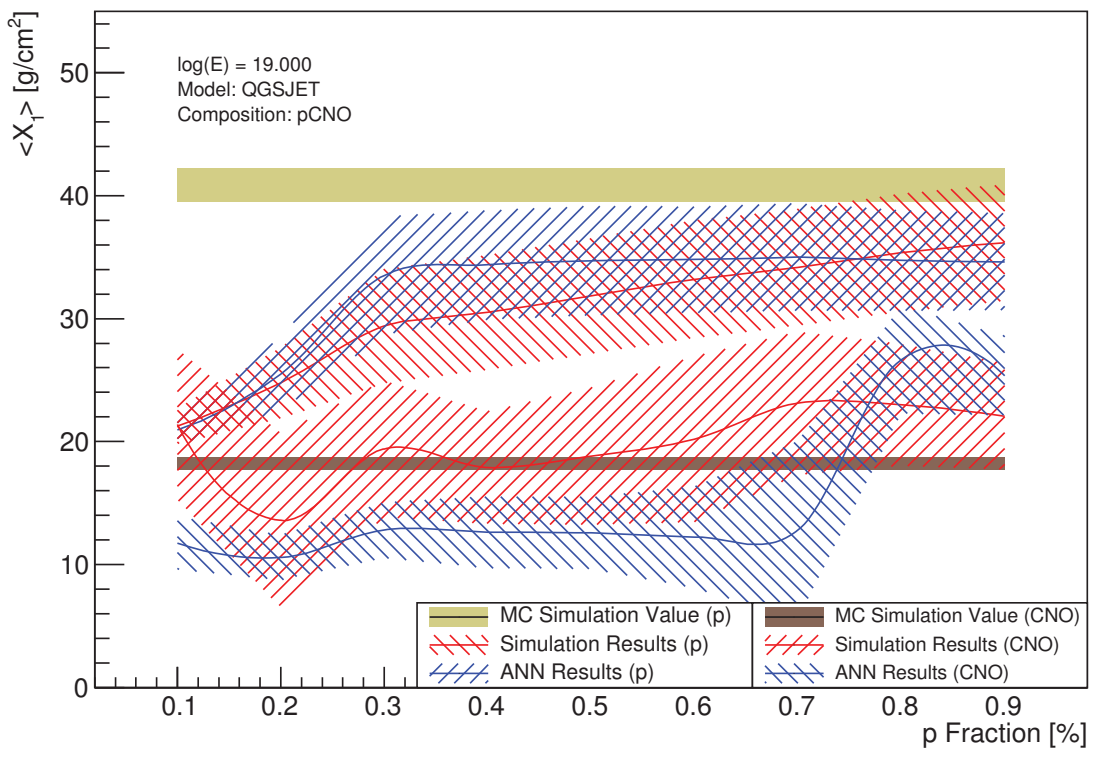

Figure 8.1: Results of pmANN+BPM for hadronic interaction model QGSJET at $E=10^{19.0} \mathrm{eV}$ for p-CNO composition

Due to uncomparable results for QGSJET, no indication of the limitation could be seen.

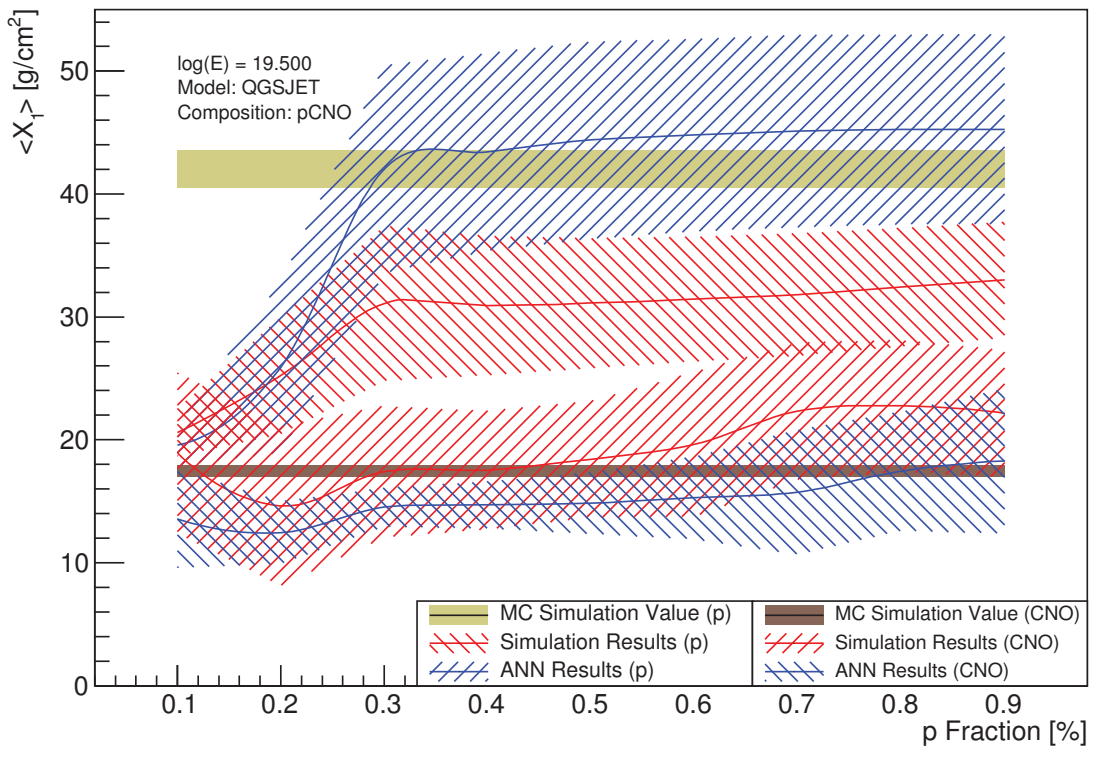

Figure 8.2: Results of pmANN+BPM for hadronic interaction model QGSJET at $\mathrm{E}=10^{19.5} \mathrm{eV}$ for p-CNO composition 


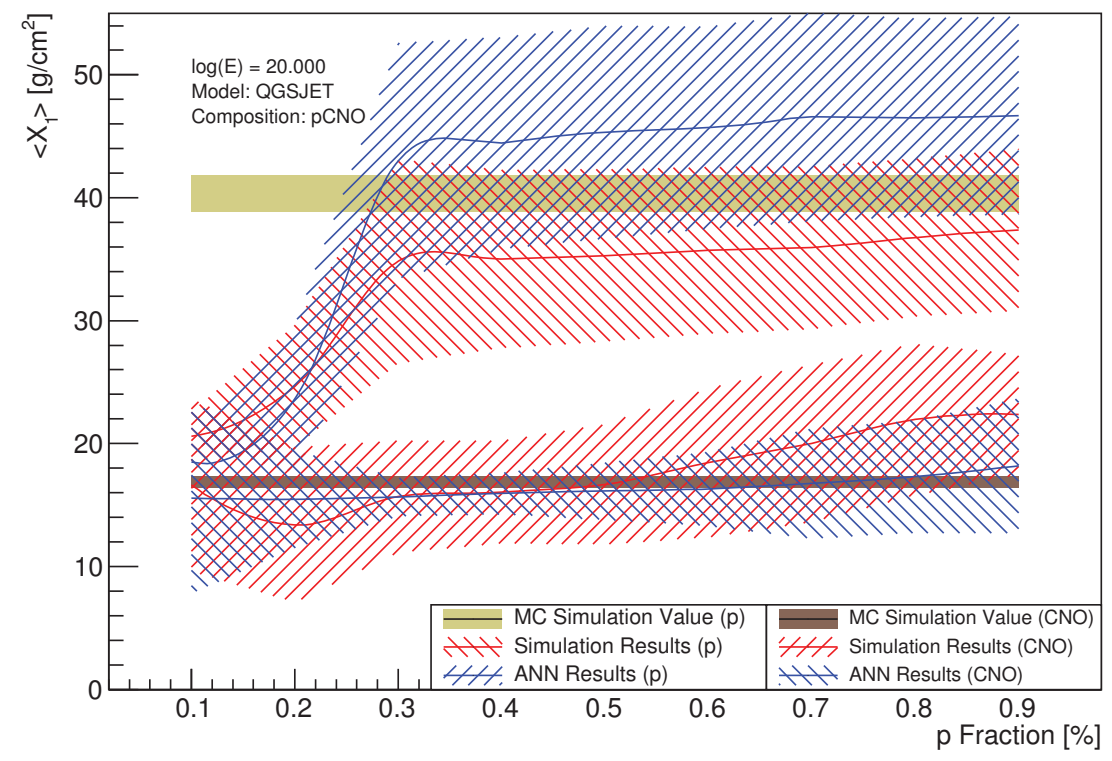

Figure 8.3: Results of pmANN+BPM for hadronic interaction model QGSJET at $E=10^{20.0} \mathrm{eV}$ for p-CNO composition

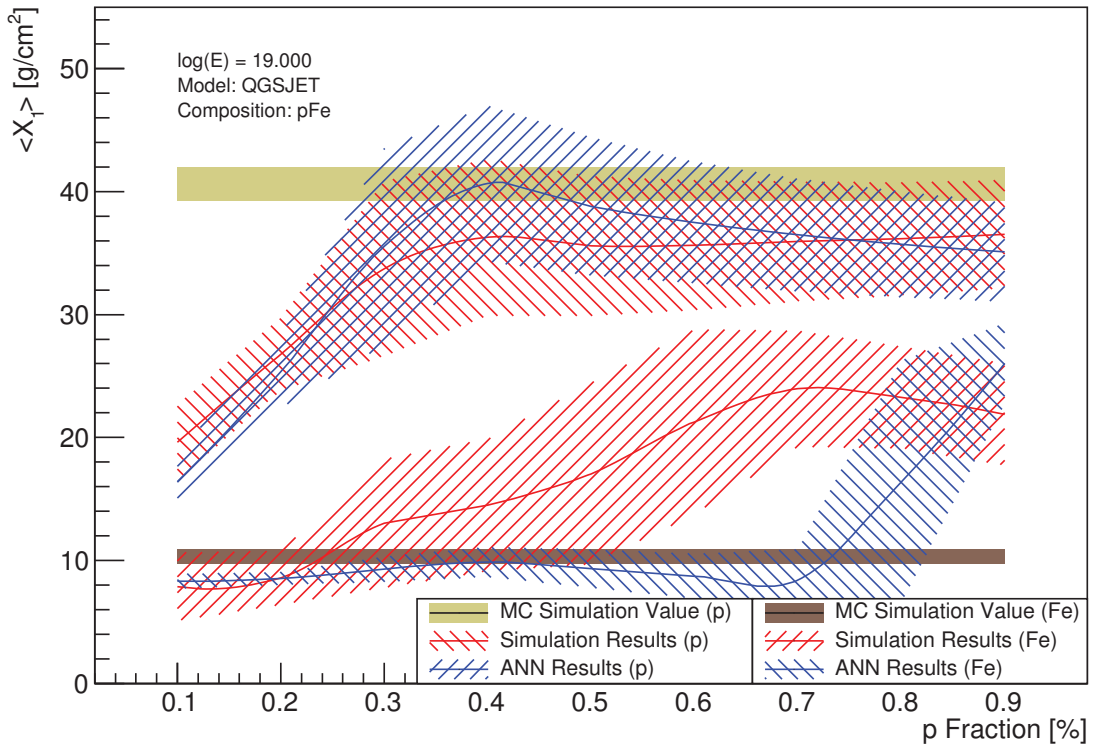

Figure 8.4: Results of pmANN+BPM for hadronic interaction model QGSJET at $E=10^{19.0} \mathrm{eV}$ for p-Fe composition 


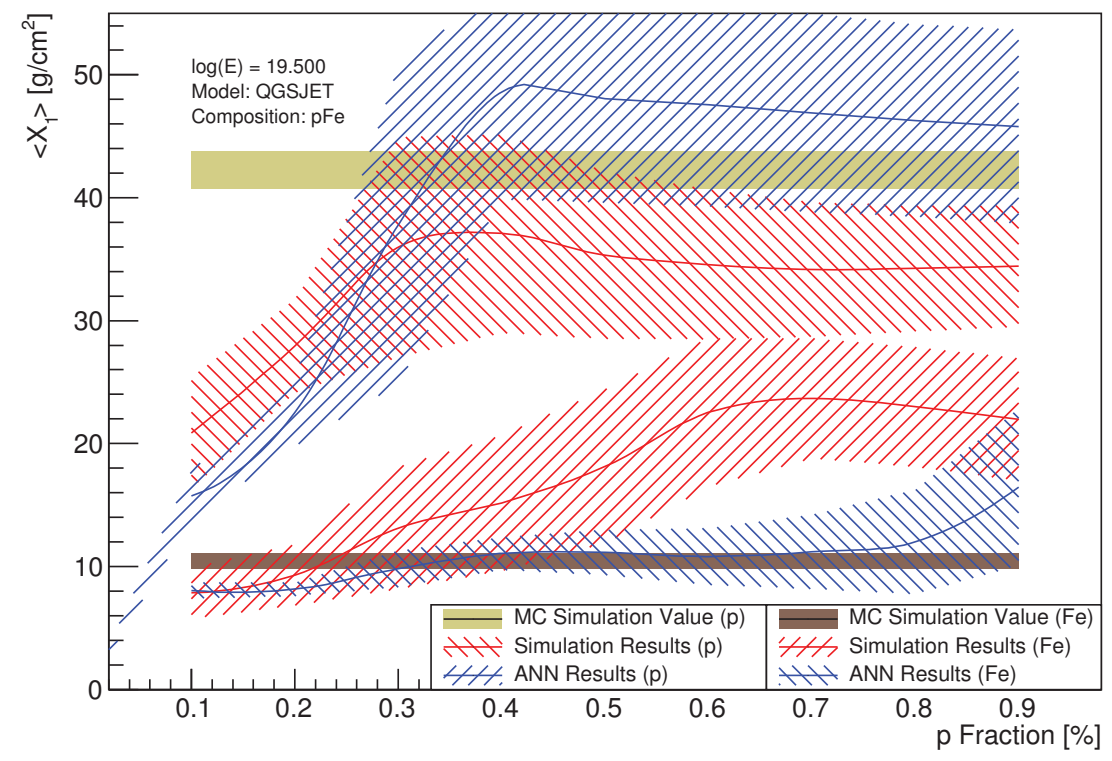

Figure 8.5: Results of pmANN+BPM for hadronic interaction model QGSJET at $E=10^{19.5} \mathrm{eV}$ for p-Fe composition

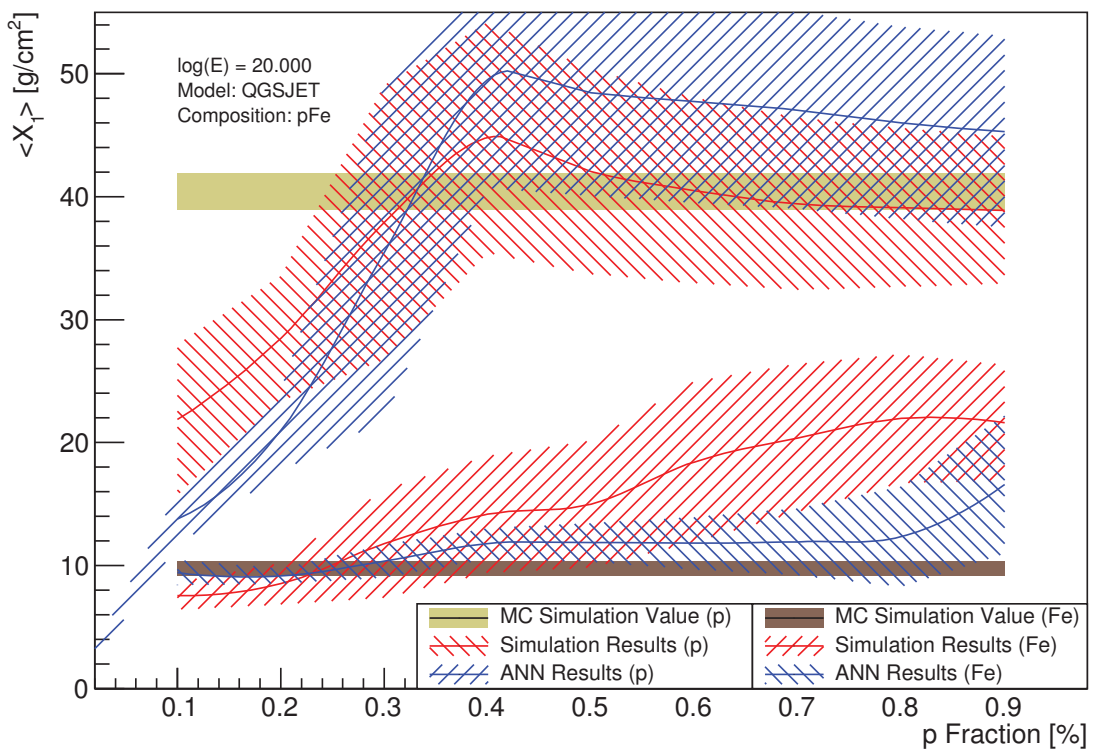

Figure 8.6: Results of pmANN+BPM for hadronic interaction model QGSJET at $E=10^{20.0} \mathrm{eV}$ for p-Fe composition 


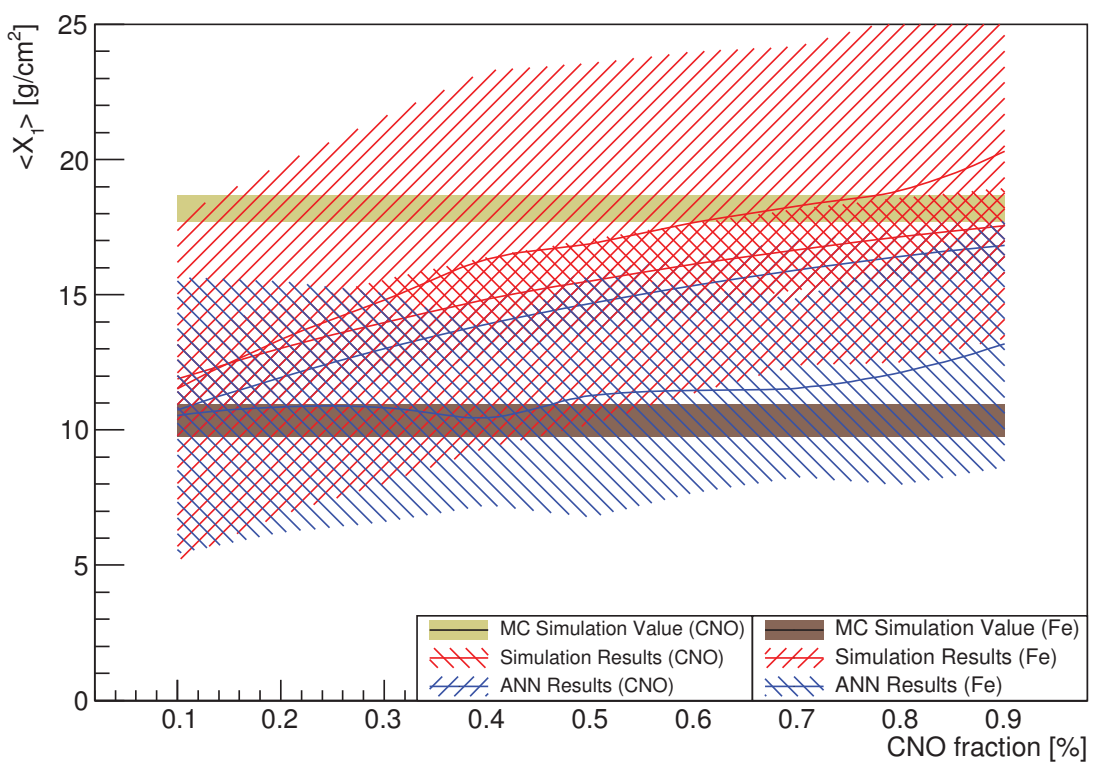

Figure 8.7: Results of pmANN+BPM for hadronic interaction model QGSJET at $E=10^{19.0} \mathrm{eV}$ for CNO-Fe composition

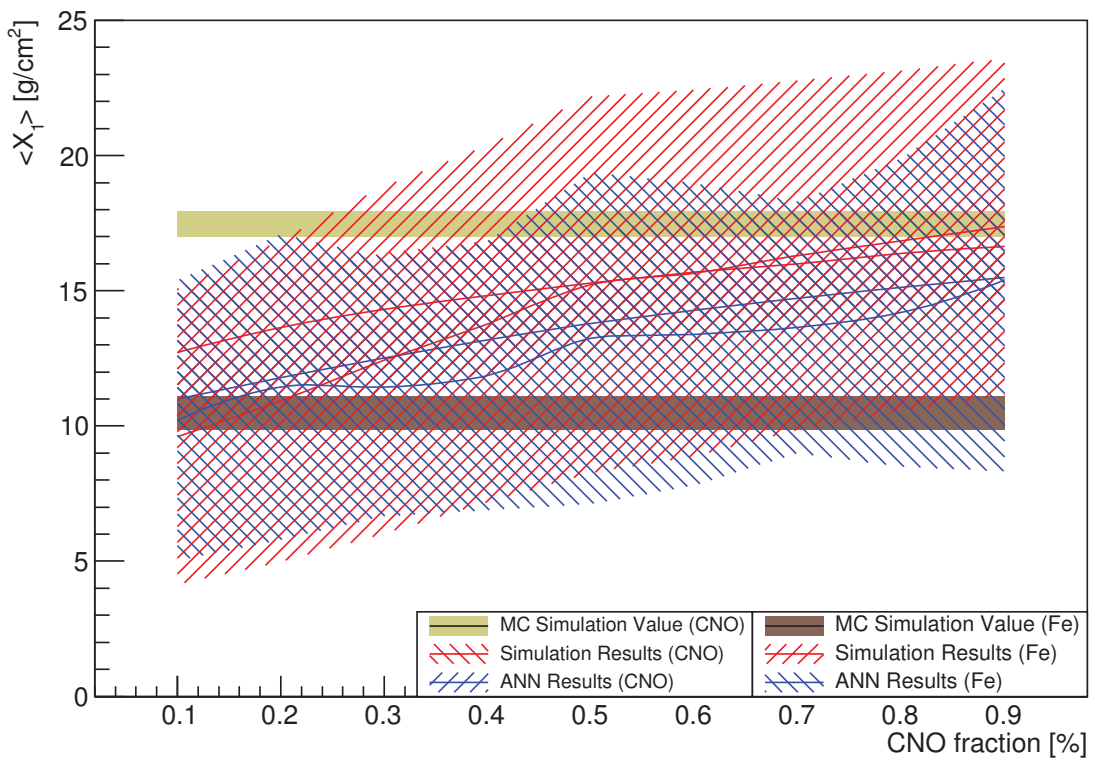

Figure 8.8: Results of pmANN+BPM for hadronic interaction model QGSJET at $\mathrm{E}=10^{19.5} \mathrm{eV}$ for CNOFe composition 


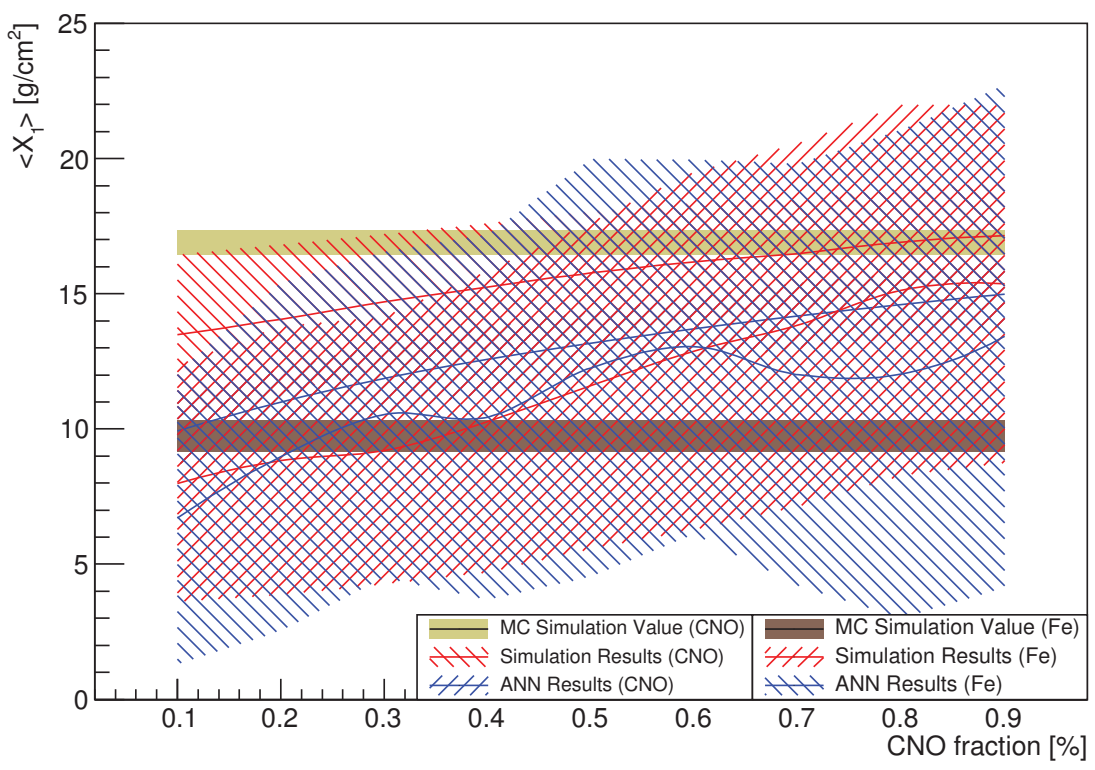

Figure 8.9: Results of pmANN+BPM for hadronic interaction model QGSJET at $E=10^{20.0} \mathrm{eV}$ for CNOFe composition

\subsection{Application of pmANN+BPM to SIBYLL Showers}

The assumed compositions were tested by changing the light particle fraction in decrements of $10 \%$. pmANN+BPM was applied to SIBYLL shower mixtures whereas only BPM was applied to the first interaction length distributions of the simulations without corrections. Results of the combined method for SIBYLL showers are shown in Figures 8.10-8.18 for proton-CNO, proton-iron and CNO-iron mixtures. Assumed light particle fractions of the compositions are shown on $\mathrm{x}$-axis in figures. The differences of the calculated simulation mean free paths and pmANN+BPM estimations with expected values of the mean free paths were shown in Appendix A.2.

For SIBYLL showers, the combined method results for $\mathrm{p}-\mathrm{CNO}$ and $\mathrm{p}-\mathrm{Fe}$ compositions agreed for $\mathrm{E}=10^{19.0}$ and $10^{19.5} \mathrm{eV}$. On the other hand, the discrency of the results with respect to Monte-Carlo simulation values at $\mathrm{E}=10^{20.0} \mathrm{eV}$ was calculated to be high, $\sim 10$ $\mathrm{g} / \mathrm{cm}^{2}$. 
The fraction chosen to switch between break points for $\mathrm{p}-\mathrm{CNO}$ and $\mathrm{p}-\mathrm{Fe}, 7 \%$, corresponds to a 50\%-50\% mixture for SIBYLL simulations. From the figures, it can be seen that pmANN+BPM predictions for the mean free path do not change until the light particle fraction drops down to $50 \%$. Below the $50 \%$ p fraction, the proton mean free path predictions were considerably different, whereas the predictions for $\mathrm{CNO}$ and $\mathrm{Fe}$ don't change as much.

Disregarding the $30 \%$ limitation mentioned and taking into account the uncertainties calculated for each case, the results were considered acceptable within $1 \sigma$. As for the high uncertainties compared to QGSJET results, they were due to the high uncertainties before the correction were applied.

A general trend about the results was the low errors of the simulation results (applied on BPM) for light particles and higher errors for heavy particle. Comparing the simulation results and pmANN+BPM results, high errors of the combined method could be addressed to pmANN. Thus, it can be concluded that pmANN performed poorly for SIBYLL showers.

The implication for the chosen fractions was also observed for CNO-Fe composition. The limitation for the fraction, $\mathrm{f}_{B P}=10 \%$ for $\mathrm{CNO}-\mathrm{Fe}$, is at $50 \% \mathrm{CNO}$ fraction in mixtures for SIBYLL. Thus, it was anticipated the method to perform poorly for cases when the CNO fraction is below the values expressed above. There is no clear indication of this effect for $\mathrm{CNO}$ mean free path predictions, and there is only a sign for Fe mean free path predictions for $\mathrm{E}=10^{19.0} \mathrm{eV}$. 


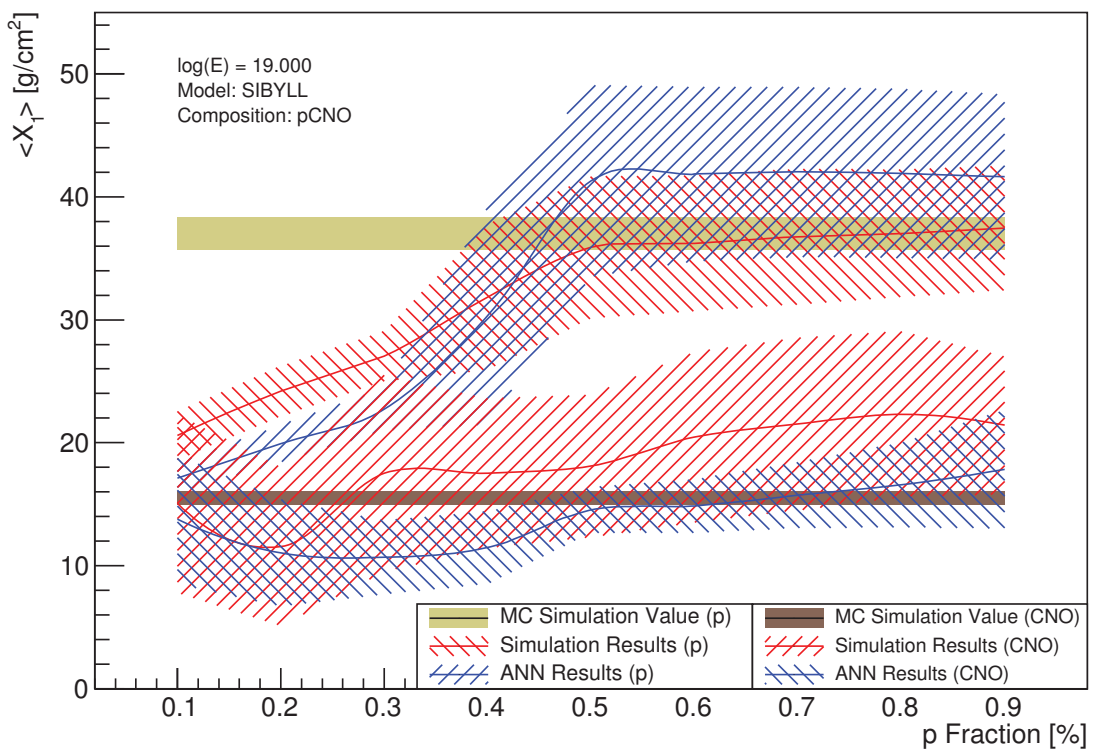

Figure 8.10: Results of $\mathrm{pmANN}+\mathrm{BPM}$ for hadronic interaction model SIBYLL at $\mathrm{E}=10^{19.0} \mathrm{eV}$ for p-CNO composition

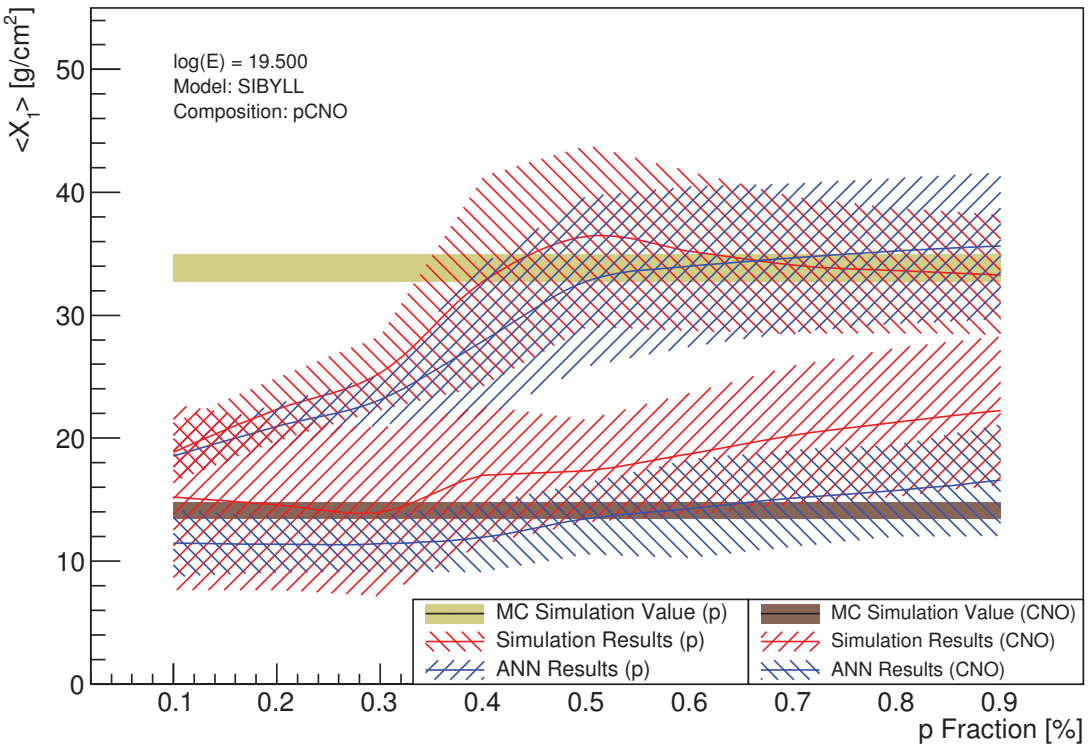

Figure 8.11: Results of pmANN+BPM for hadronic interaction model SIBYLL at $E=10^{19.5} \mathrm{eV}$ for p-CNO composition 


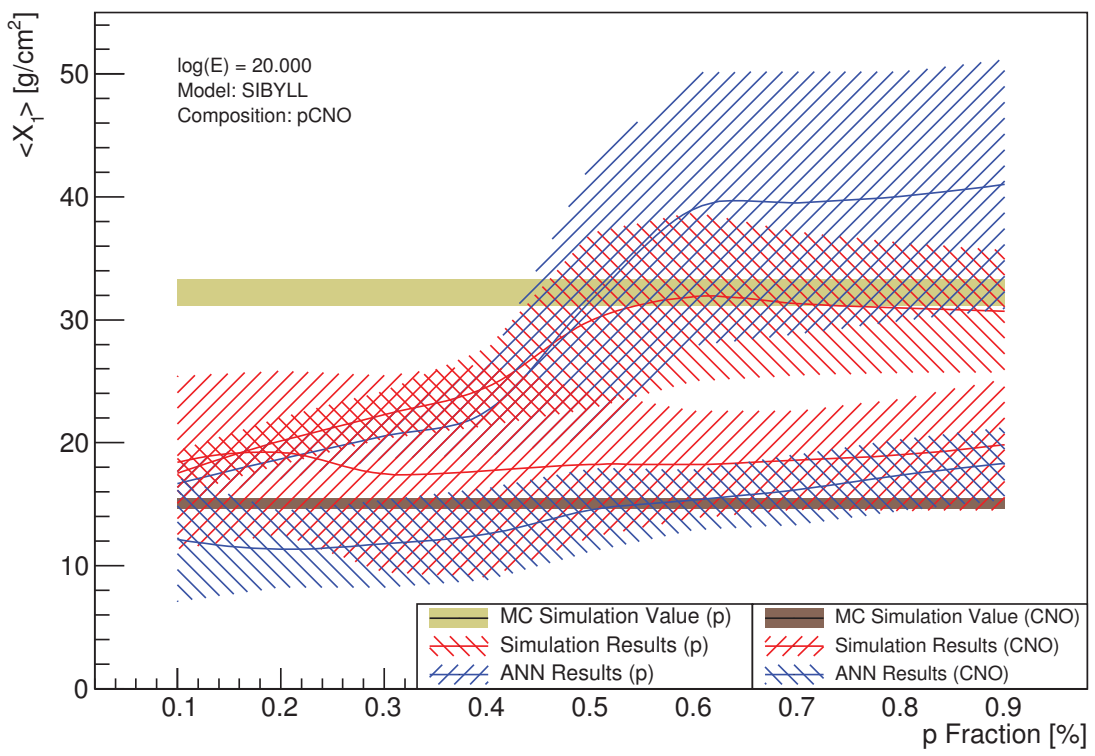

Figure 8.12: Results of $\mathrm{pmANN}+\mathrm{BPM}$ for hadronic interaction model SIBYLL at $\mathrm{E}=10^{20.0} \mathrm{eV}$ for p-CNO composition

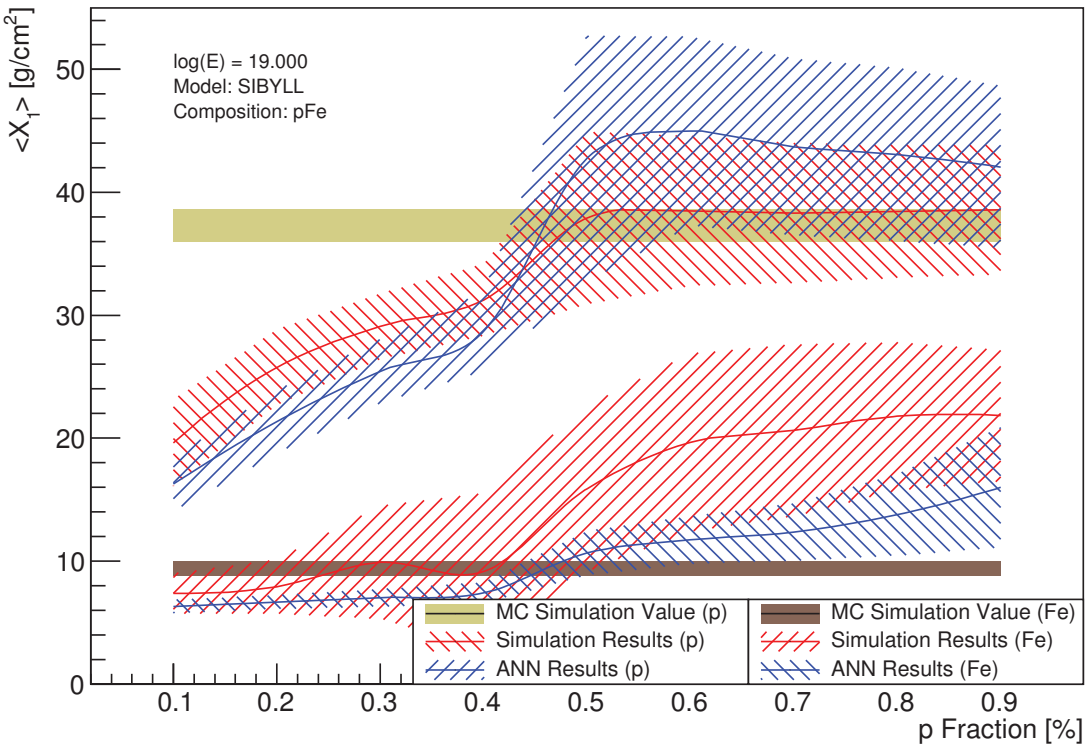

Figure 8.13: Results of pmANN+BPM for hadronic interaction model SIBYLL at $E=10^{19.0} \mathrm{eV}$ for p-Fe composition 


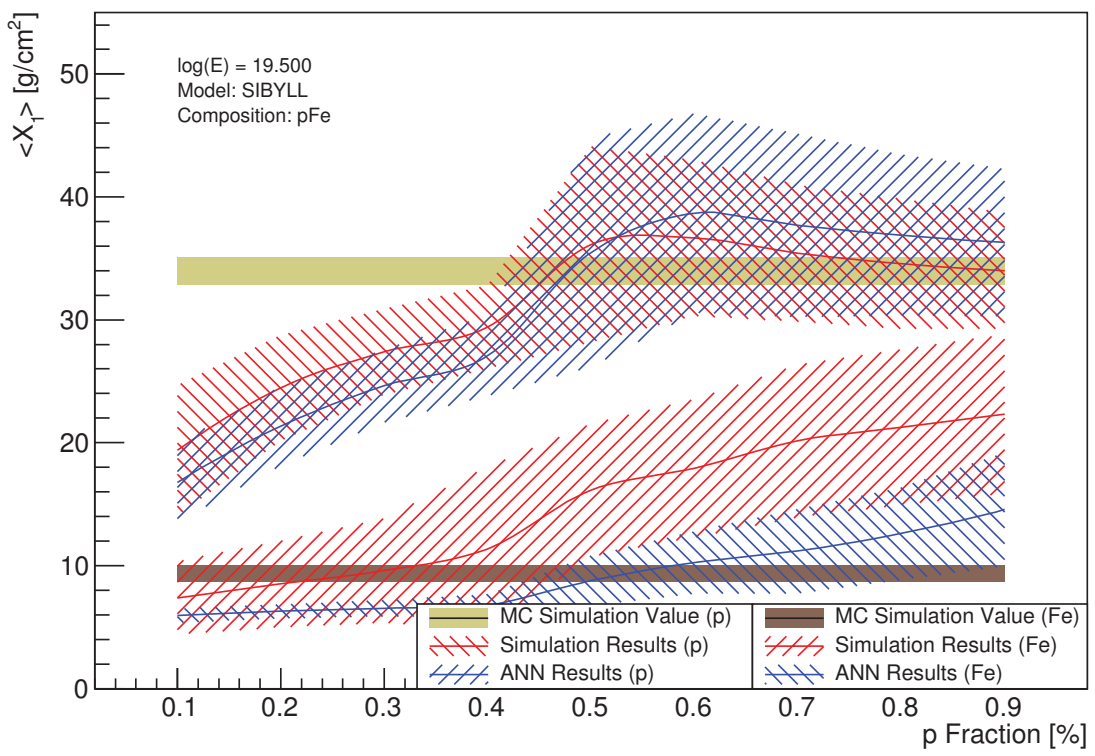

Figure 8.14: Results of pmANN+BPM for hadronic interaction model SIBYLL at $E=10^{19.5} \mathrm{eV}$ for p-Fe composition

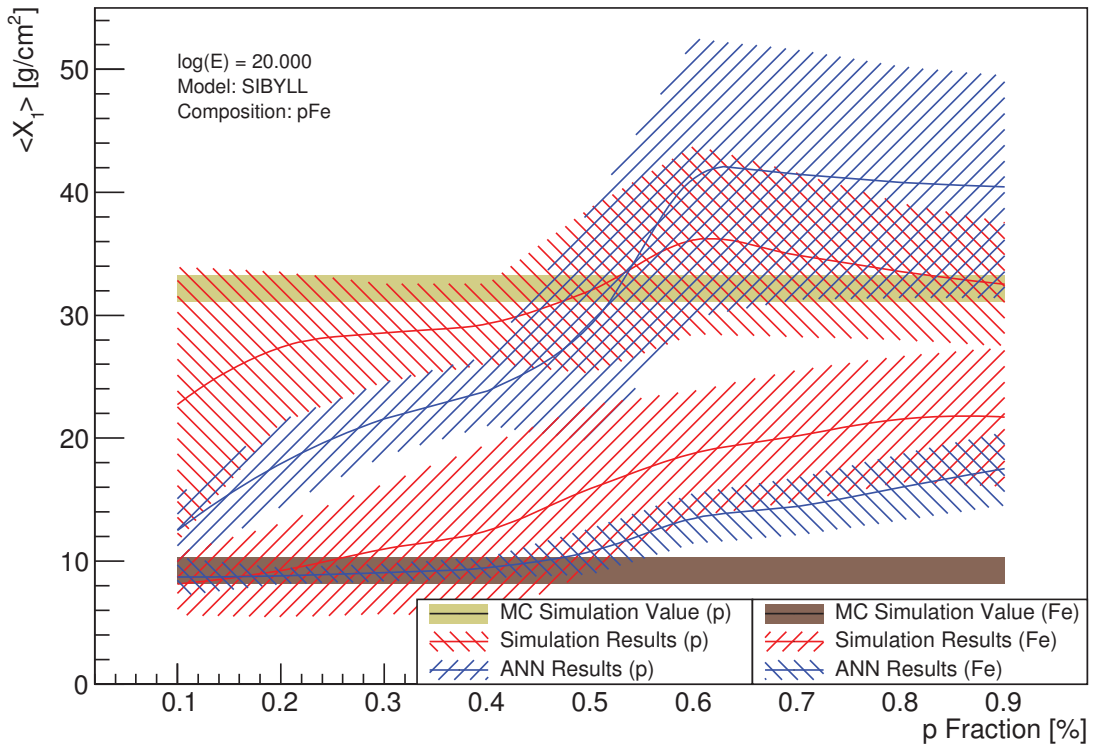

Figure 8.15: Results of pmANN+BPM for hadronic interaction model SIBYLL at $E=10^{20.0} \mathrm{eV}$ for p-Fe composition 


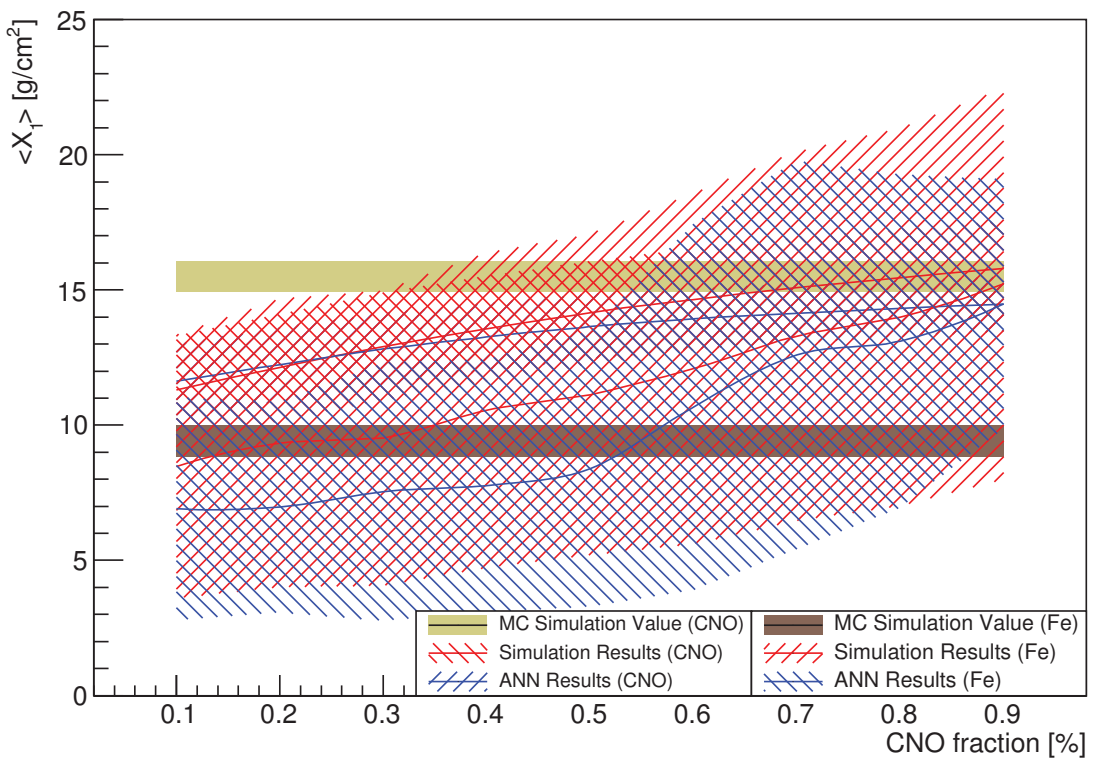

Figure 8.16: Results of pmANN+BPM for hadronic interaction model SIBYLL at $E=10^{19.0} \mathrm{eV}$ for CNO-Fe composition

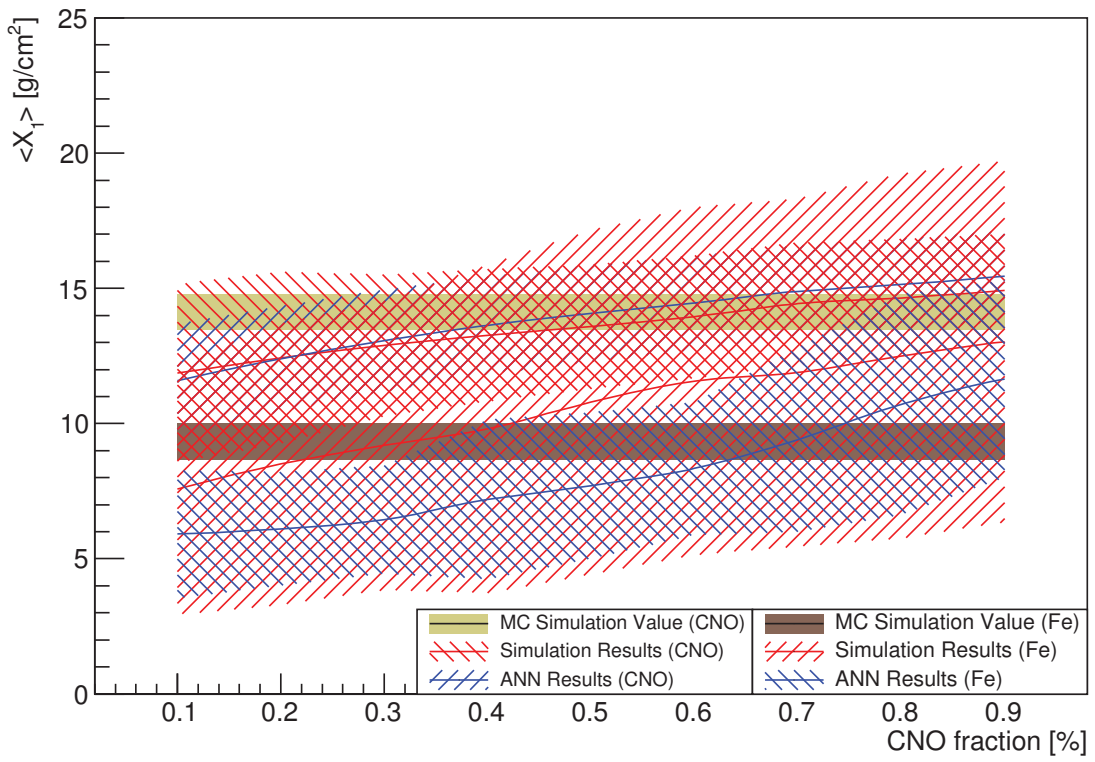

Figure 8.17: Results of PmANN+BPM for hadronic interaction model SIBYLL at $E=10^{19.5} \mathrm{eV}$ for CNOFe composition 


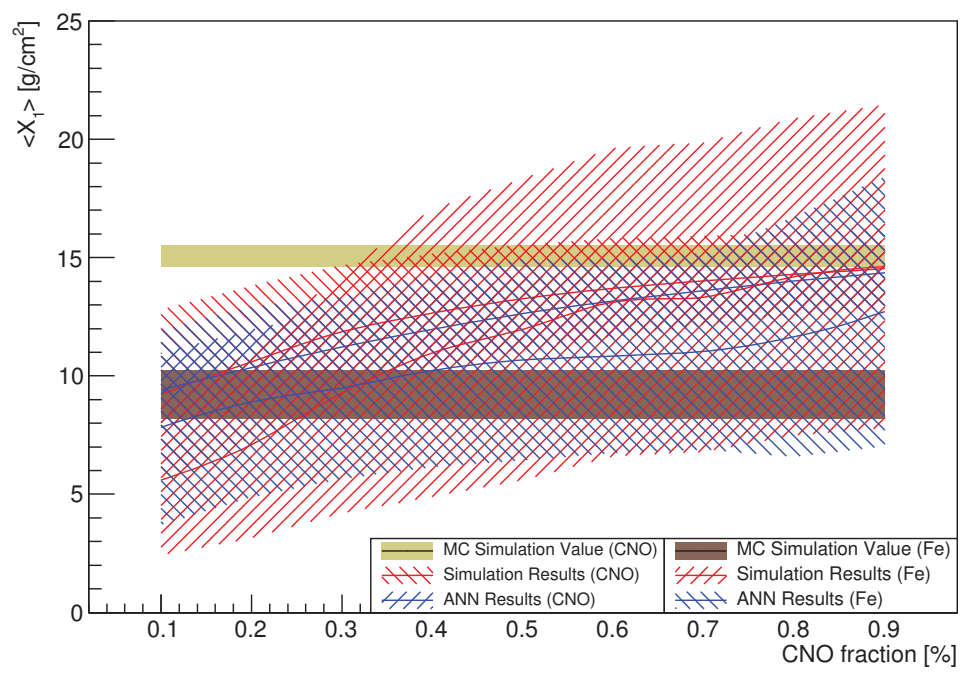

Figure 8.18: Results of $\mathrm{pmANN}+\mathrm{BPM}$ for hadronic interaction model SIBYLL at $\mathrm{E}=10^{20.0} \mathrm{eV}$ for CNOFe composition

\subsection{Application of pmANN+BPM to EPOS Showers}

The assumed compositions were tested by changing the light particle fraction in decrements of $10 \%$. pmANN+BPM was applied to EPOS shower mixtures whereas only BPM was applied to first interaction length distributions of the simulations without corrections. Results of the combined method for EPOS showers are shown in Figures 8.19-8.27. Assumed light particle fractions of the compositions are shown on $\mathrm{x}$-axis in figures. Differences of the calculated simulation mean free paths and pmANN+BPM estimations with expected values of the mean free paths were shown in Appendix A.3.

The effect of the $30 \%$ limitation explained in the results of QGSJET01 showers is also prominent for EPOS showers. The error on the estimation increases drastically to $\sim 10$ $\mathrm{g} / \mathrm{cm}^{2}$ for the proton mean free path independent of the energy when the proton fraction iss below $30 \%$. Unlike the proton mean free path predictions, the limitation for the heavy particles ( $\mathrm{CNO}$ and $\mathrm{Fe}$ ) are not strongly evident.

The results for CNO-Fe composition show good predictions for $\mathrm{CNO}$ at high CNO fractions. The performance of the predictions gets worse when CNO fraction decreases. Similarly, the Fe mean free path predictions follows a similar trend. 


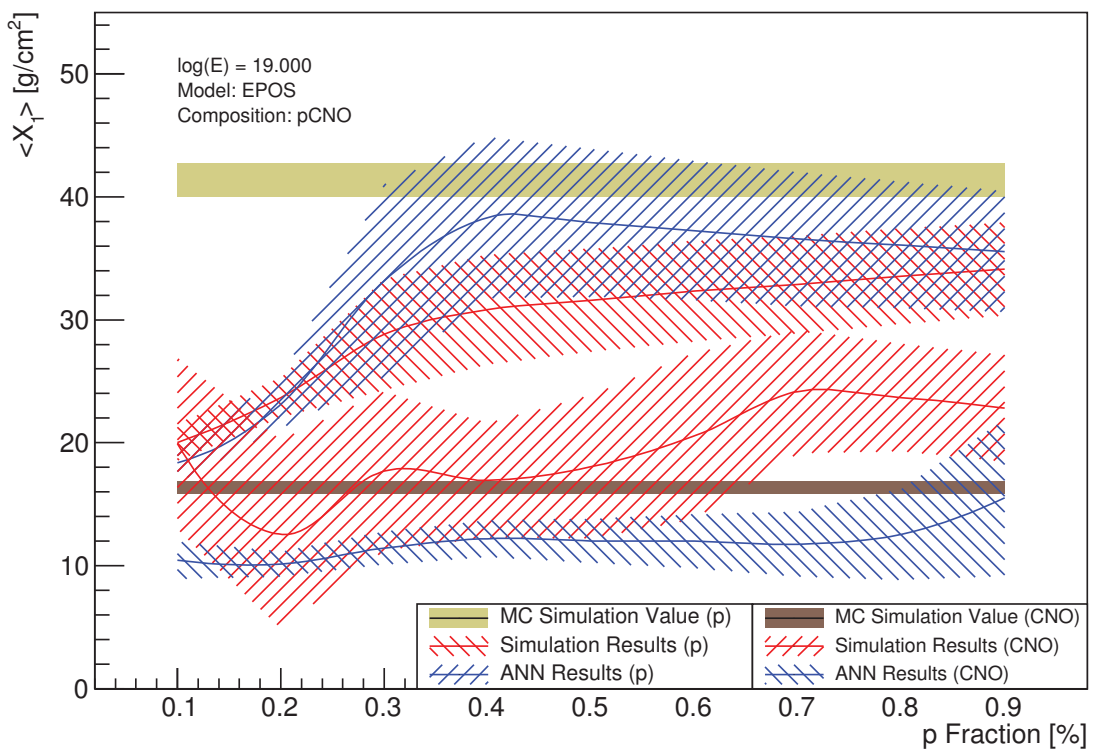

Figure 8.19: Results of $p m A N N+B P M$ for hadronic interaction model $E P O S$ at $E=10^{19.0} \mathrm{eV}$ for p-CNO composition

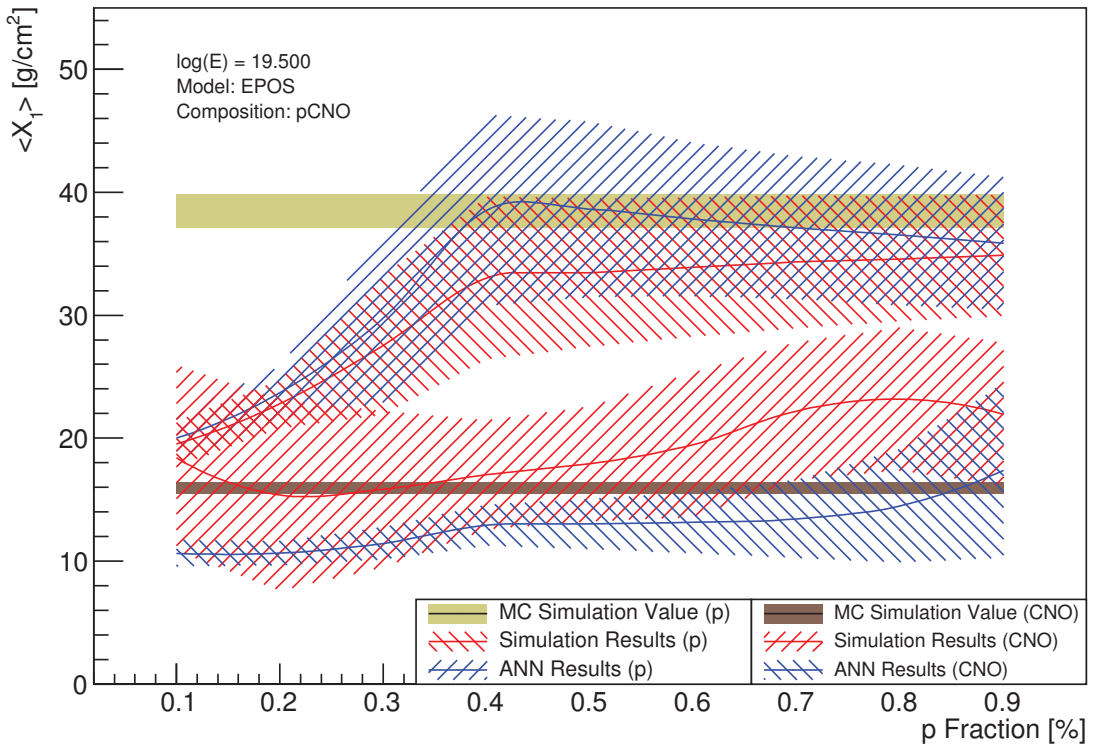

Figure 8.20: Results of pmANN+BPM for hadronic interaction model $E P O S$ at $E=10^{19.5} \mathrm{eV}$ for p-CNO composition 


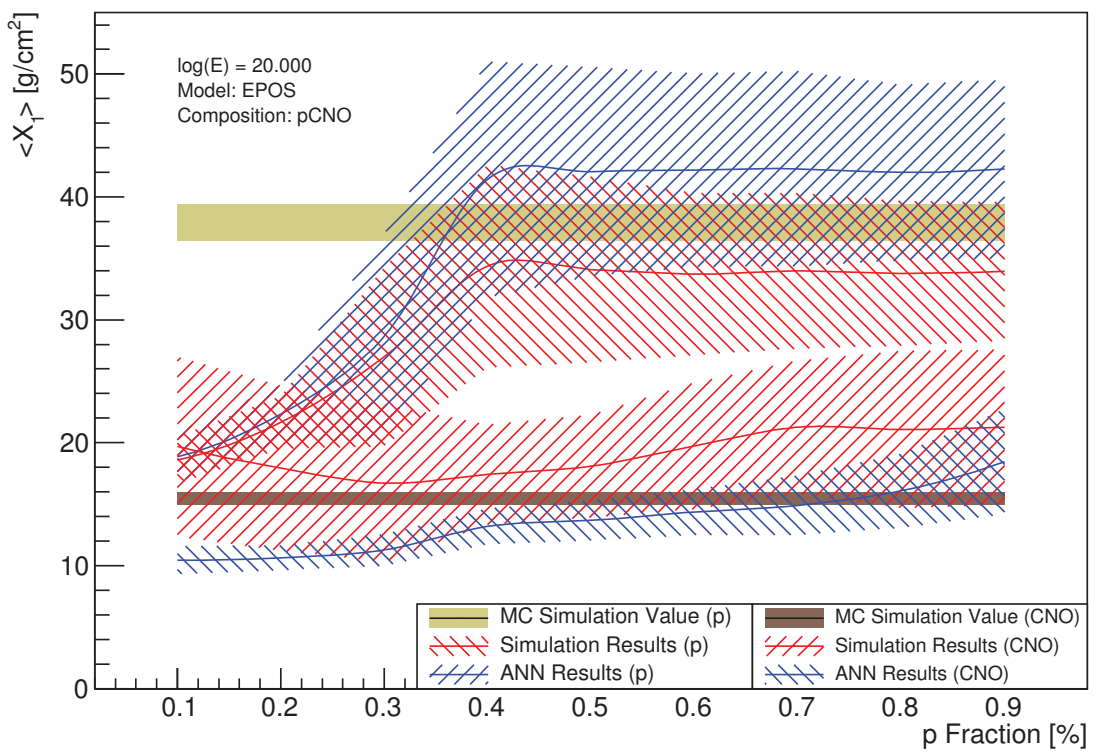

Figure 8.21: Results of $p m A N N+B P M$ for hadronic interaction model $E P O S$ at $E=10^{20.0} \mathrm{eV}$ for p-CNO composition

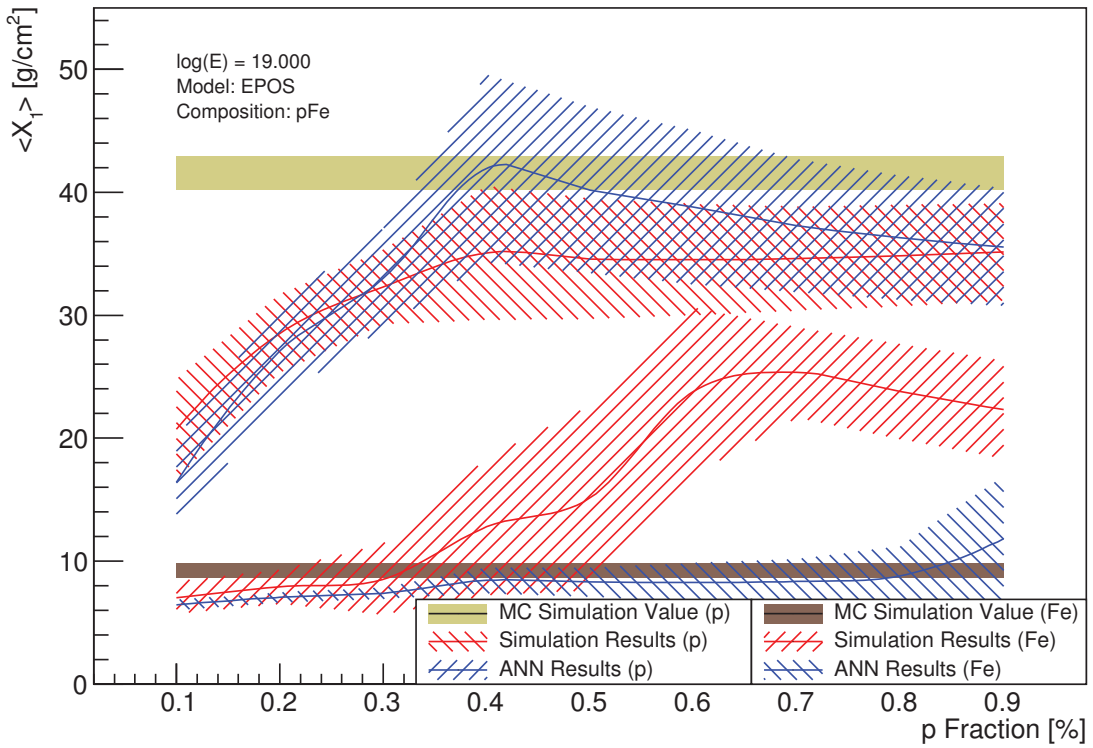

Figure 8.22: Results of pmANN+BPM for hadronic interaction model $E P O S$ at $E=10^{19.0} \mathrm{eV}$ for p-Fe composition 


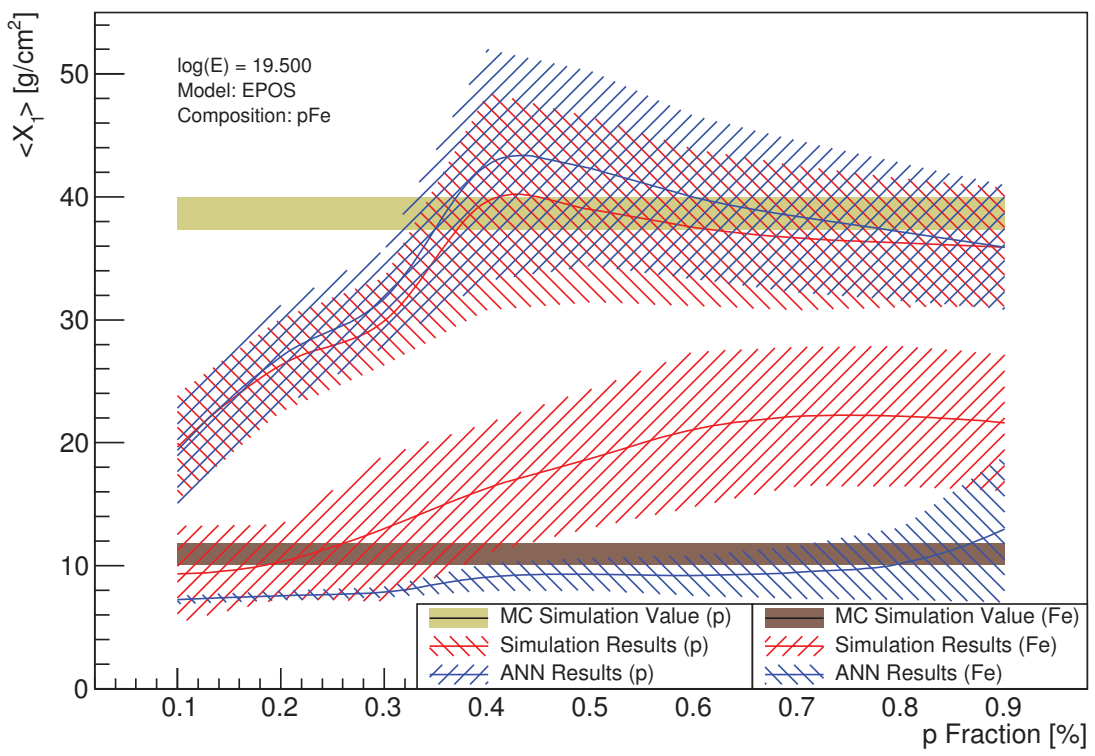

Figure 8.23: Results of pmANN+BPM for hadronic interaction model $E P O S$ at $E=10^{19.5} \mathrm{eV}$ for p-Fe composition

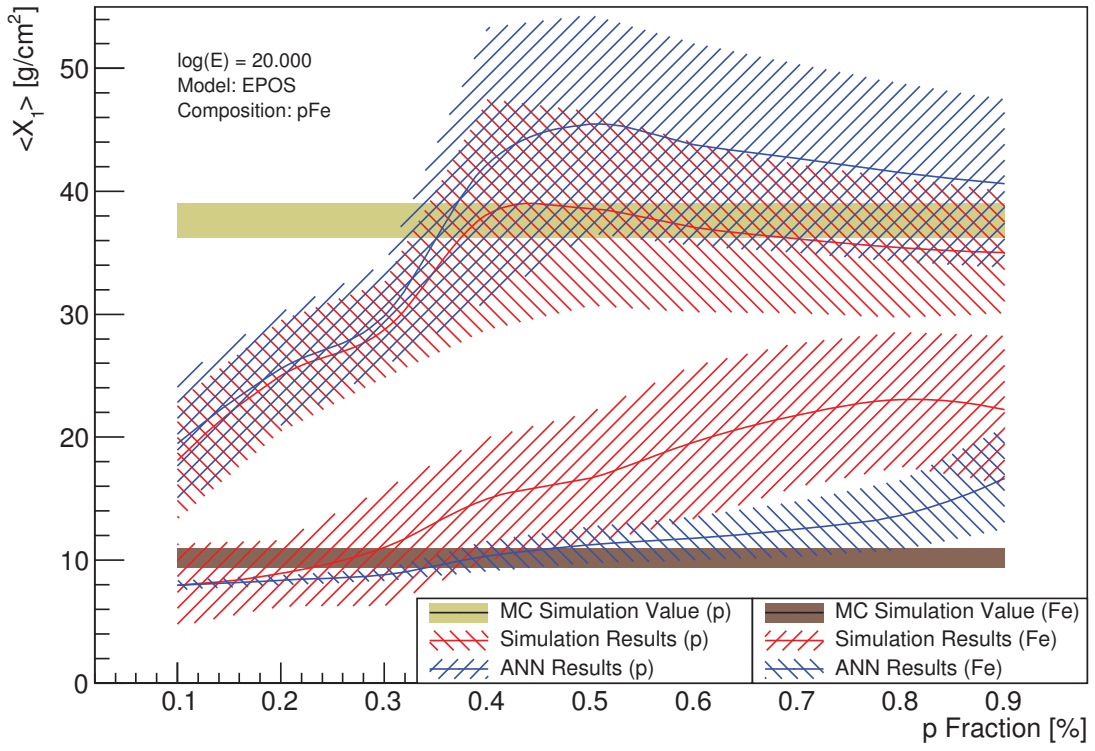

Figure 8.24: Results of $\mathrm{pmANN}+\mathrm{BPM}$ for hadronic interaction model $\mathrm{EPOS}$ at $\mathrm{E}=10^{20.0} \mathrm{eV}$ for p-Fe composition 


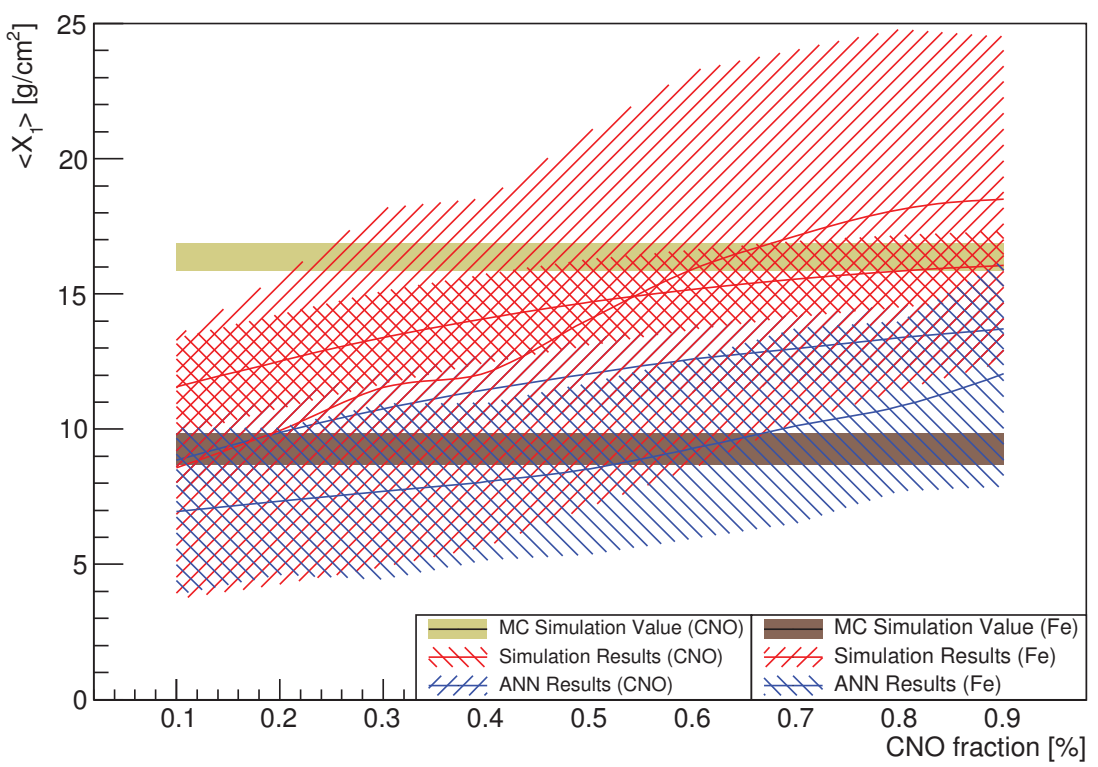

Figure 8.25: Results of pmANN+BPM for hadronic interaction model $\mathrm{EPOS}$ at $\mathrm{E}=10^{19.0} \mathrm{eV}$ for CNO-Fe composition

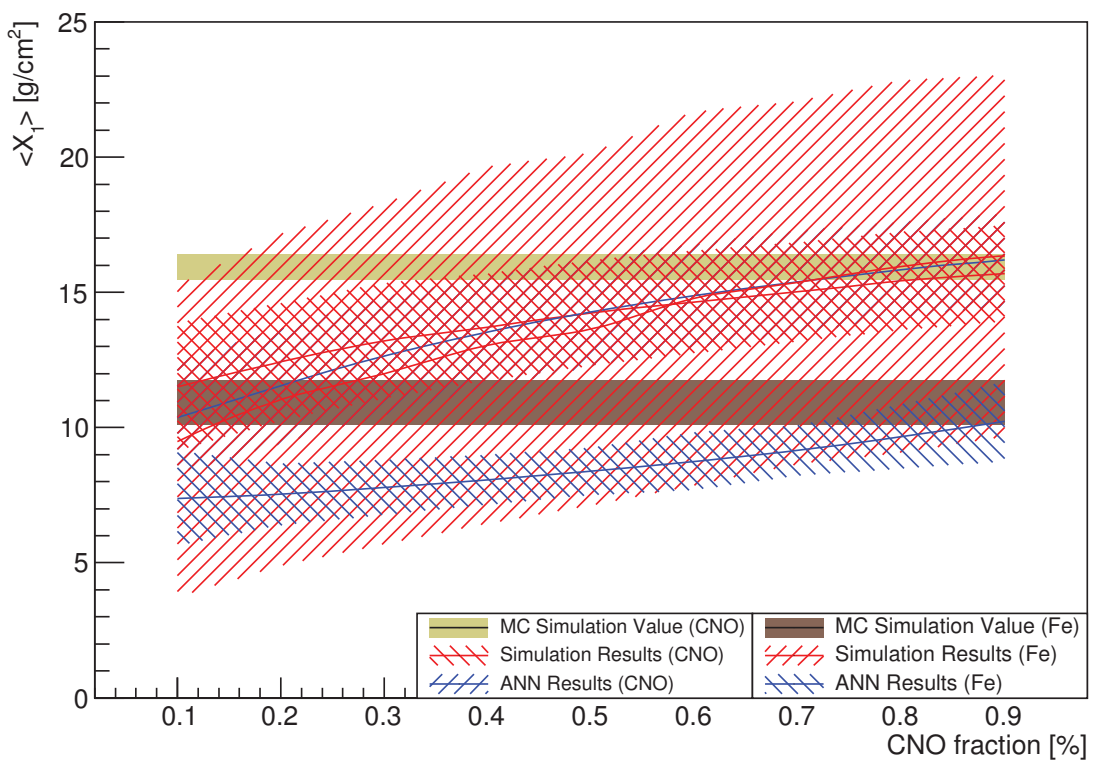

Figure 8.26: Results of $\mathrm{pmANN}+\mathrm{BPM}$ for hadronic interaction model $\mathrm{EPOS}$ at $\mathrm{E}=10^{19.5} \mathrm{eV}$ for CNOFe composition 


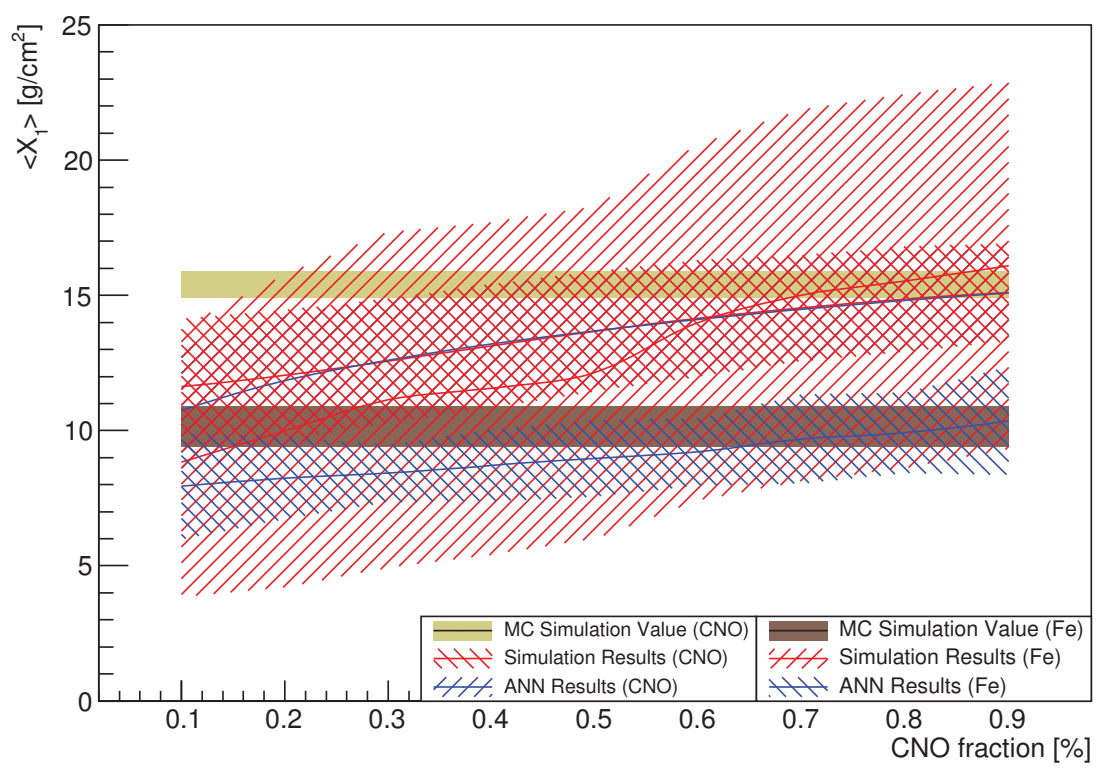

Figure 8.27: Results of pmANN+BPM for hadronic interaction model $\mathrm{EPOS}$ at $\mathrm{E}=10^{20.0} \mathrm{eV}$ for CNOFe composition 


\section{Chapter 9 Conclusion and Outlook}

An artificial neural network (pmANN) was trained for the prediction of the first interaction lengths. For this purpose, proton, CNO and iron showers generated by QGSJET, SIBYLL and EPOS at energies ranging from $10^{18.5}$ to $10^{20}$ were fed to the neural network. It can be said that the training was done with an effective hadronic interaction model. The results of the predictions were used to demonstrate the capability of the model to compute the mean free path of the primary particles. Detailed procedure and results were given in Chapter 5.

The sources of cosmic rays is not yet well known at the ultra high energies. Thus, the application of pmANN would not work to compute the mean free paths of the cosmic rays. In order to overcome this difficulty, an algebraic method relying on Monte-Carlo calculation was carried out. The results of the method were given in Chapter 6.

The results of each method showed biases to some extent. When the method were employed consecutively, the biases of each method were propagated to the overall result. The combined method results were tabulated in Chapter 7.

For the combined method to work, the muon production depth profile is needed. Unfortunately, despite the MPD reconstruction method discussed in 4.2.1, reconstruction of the MPD profile did not yield results comparable to the original MPD. Thus, the effect of the detector simulation (event reconstruction) could not be performed. The combined method was not applied to any reconstructed events, thusly. Upon the availability of a method that will perform the task, the combined method will be applied to attain important information for particle physics at ultra high energies. 


\section{References}

[1] A. De Angelis, "Cosmic rays: studies and measurements before 1912," Nuclear Physics B - Proceedings Supplements, vol. 239-240, pp. 3-10, June 2013.

[2] I. V. Dorman and L. I. Dorman, "How cosmic rays were discovered and why they received this misnomer," Advances in Space Research, vol. 53, pp. 1388-1404, May 2014.

[3] J. A. Angelo, Nuclear Technology. Greenwood Press, 2004.

[4] V. F. Hess, "Über beobachtungen der durchdringenden strahlung bei sieben freiballonfahrt," Physikalische Zeitschrift, vol. 13, pp. 1084-1091, 1912.

[5] C. Grupen, "The history of cosmic ray studies after hess," Nuclear Physics B - Proceedings Supplements, vol. 239-240, pp. 19-25, June 2013.

[6] W. Kolhorster Physikalische Zeitschrift, vol. 14, p. 1153, 1913.

[7] P. Carlson, “A century of cosmic rays," Physics Today, vol. 65, pp. 30-36, Feb. 2012.

[8] C. Grupen, "Early developments: Particle physics aspects of cosmic rays," Astroparticle Physics, vol. 53, pp. 86-90, Jan. 2014.

[9] C. D. Anderson, “The positive electron,” Physical Review, vol. 43, pp. 491-494, Mar. 1933.

[10] "1938, Pierre Auger." http://www.auger.org/cosmic_rays/1938. html. Online, Accessed: 2013-06-14.

[11] K.-H. Kampert and A. A. Watson, "Extensive air showers and ultra high-energy cosmic rays: A historical review," arXiv:1207.4827 [astro-ph, physics:physics], July 2012. arXiv: 1207.4827.

[12] J. Linsley, "Evidence for a primary cosmic-ray particle with energy $1020 \mathrm{eV}$," Physical Review Letters, vol. 10, pp. 146-148, Feb. 1963.

[13] E. S. Seo, "Direct measurements of cosmic rays using balloon borne experiments," Astroparticle Physics, vol. 39-40, pp. 76-87, Dec. 2012. 
[14] T. K. Gaisser, Cosmic Rays and Particle Physics. Oxford Master Series in Particle Physics, Astrophysics and Cosmology, Cambridge University Press, 1st edition ed., 1990.

[15] A. A. Ivanov and f. t. Y. a. group, "A search for extragalactic sources of ultrahighenergy cosmic rays," JETP Letters, vol. 87, pp. 185-189, Apr. 2008. arXiv: 0803.0612 .

[16] N. Chiba, K. Hashimoto, N. Hayashida, K. Honda, M. Honda, N. Inoue, F. Kakimoto, K. Kamata, S. Kawaguchi, N. Kawasumi, Y. Matsubara, K. Murakami, M. Nagano, S. Ogio, H. Ohoka, T. Saito, Y. Sakuma, I. Tsushima, M. Teshima, T. Umezawa, S. Yoshida, and H. Yoshii, "Akeno giant air shower array (AGASA) covering 100 km2 area," Nuclear Instruments and Methods in Physics Research Section A: Accelerators, Spectrometers, Detectors and Associated Equipment, vol. 311, pp. 338-349, Jan. 1992.

[17] R. M. Baltrusaitis, R. Cady, G. L. Cassiday, R. Cooperv, J. W. Elbert, P. R. Gerhardy, S. Ko, E. C. Loh, M. Salamon, D. Steck, and P. Sokolsky, "The utah fly's eye detector," Nuclear Instruments and Methods in Physics Research Section A: Accelerators, Spectrometers, Detectors and Associated Equipment, vol. 240, pp. 410-428, Oct. 1985.

[18] H. D. Engel R. and P. T., "Extensive air showers and hadronic interactions at high energy," Annual Review of Nuclear and Particle Science, vol. 61, pp. 467-489, 2011.

[19] D. J. Bird, S. C. Corbató, H. Y. Dai, B. R. Dawson, J. W. Elbert, T. K. Gaisser, K. D. Green, M. A. Huang, D. B. Kieda, S. Ko, C. G. Larsen, E. C. Loh, M. Luo, M. H. Salamon, D. Smith, P. Sokolsky, P. Sommers, T. Stanev, J. K. K. Tang, S. B. Thomas, and S. Tilav, "Evidence for correlated changes in the spectrum and composition of cosmic rays at extremely high energies," Physical Review Letters, vol. 71, pp. 34013404, Nov. 1993.

[20] W. D. Apel, J. C. Arteaga-Velàzquez, K. Bekk, M. Bertaina, J. Blümer, H. Bozdog, I. M. Brancus, E. Cantoni, A. Chiavassa, F. Cossavella, K. Daumiller, V. de Souza, F. Di Pierro, P. Doll, R. Engel, J. Engler, M. Finger, B. Fuchs, D. Fuhrmann, H. J. Gils, R. Glasstetter, C. Grupen, A. Haungs, D. Heck, J. R. Hörandel, D. Huber, T. Huege, K.-H. Kampert, D. Kang, H. O. Klages, K. Link, P. Łuczak, M. Ludwig, H. J. Mathes, H. J. Mayer, M. Melissas, J. Milke, B. Mitrica, C. Morello, J. Oehlschläger, S. Ostapchenko, N. Palmieri, M. Petcu, T. Pierog, H. Rebel, M. Roth, 
H. Schieler, S. Schoo, F. G. Schröder, O. Sima, G. Toma, G. C. Trinchero, H. Ulrich, A. Weindl, J. Wochele, M. Wommer, and J. Zabierowski, "Ankle-like feature in the energy spectrum of light elements of cosmic rays observed with KASCADE-grande," Physical Review D, vol. 87, Apr. 2013. arXiv: 1304.7114.

[21] K. Greisen, "End to the cosmic-ray spectrum?," Physical Review Letters, vol. 16, pp. 748-750, Apr. 1966.

[22] G. T. Zatsepin and V. A. Kuz'min, "Upper limit of the spectrum of cosmic rays," Soviet Journal of Experimental and Theoretical Physics Letters, vol. 4, p. 78, Aug. 1966.

[23] H. Collaboration, "First observation of the greisen-zatsepin-kuzmin suppression," Physical Review Letters, vol. 100, Mar. 2008. arXiv: astro-ph/0703099.

[24] The Pierre Auger Collaboration, "Observation of the suppression of the flux of cosmic rays above $4 \times 10^{\wedge}\{19\}$ eV," Physical Review Letters, vol. 101, p. 061101, Aug. 2008.

[25] D. Allard, "Extragalactic propagation of ultrahigh energy cosmic-rays," Astroparticle Physics, vol. 39-40, pp. 33-43, Dec. 2012.

[26] J. W. Cronin, “The highest-energy cosmic rays," Nuclear Physics B - Proceedings Supplements, vol. 138, pp. 465-491, Jan. 2005.

[27] E. Fermi, “On the origin of the cosmic radiation,” Physical Review, vol. 75, pp. 11691174, Apr. 1949.

[28] H. J. de Vega and N. Sanchez, "Extreme energy cosmic rays: Bottom-up vs. top-down scenarii," arXiv:astro-ph/0301039, Jan. 2003. arXiv: astro-ph/0301039.

[29] M. Kachelriess, "The rise and fall of top-down models as main UHECR sources," arXiv:0810.3017 [astro-ph, physics:hep-ph], Oct. 2008. arXiv: 0810.3017.

[30] D. V. Semikoz and f. t. P. A. Collaboration, "Constraints on top-down models for the origin of UHECRs from the pierre auger observatory data," arXiv:0706.2960 [astroph], June 2007. arXiv: 0706.2960.

[31] D. Semikoz and G. Sigl, "Ultra-high energy neutrino fluxes: New constraints and implications," Journal of Cosmology and Astroparticle Physics, vol. 2004, pp. $003-$ 003, Apr. 2004. arXiv: hep-ph/0309328. 
[32] P. L. Biermann, "The origin of cosmic rays," Nuclear Physics B - Proceedings Supplements, vol. 43, pp. 221-228, June 1995.

[33] A. Achterberg, Y. A. Gallant, J. G. Kirk, and A. W. Guthmann, "Particle acceleration by ultra-relativistic shocks: theory and simulations," Monthly Notices of the Royal Astronomical Society, vol. 328, pp. 393-408, Dec. 2001. arXiv: astro-ph/0107530.

[34] M. A. Malkov and P. H. Diamond, "Modern theory of fermi acceleration: a new challenge to plasma physics," Physics of Plasmas, vol. 8, no. 5, p. 2401, 2001. arXiv: astro-ph/0102373.

[35] M. Ostrowski, "Mechanisms and sites of ultra high energy cosmic ray origin," Astroparticle Physics, vol. 18, pp. 229-236, Dec. 2002. arXiv: astro-ph/0101053.

[36] the Piere Auger Observatory, "Correlation of the highest-energy cosmic rays with nearby extragalactic objects," Science, vol. 318, pp. 938-943, Nov. 2007.

[37] E. Roulet, "Latest results from the pierre auger observatory," arXiv:1101.1825 [astroph, physics:hep-ph], Jan. 2011. arXiv: 1101.1825.

[38] P. A. Collaboration, "Depth of maximum of air-shower profiles at the pierre auger observatory: Composition implications," arXiv:1409.5083 [astro-ph], Sept. 2014. arXiv: 1409.5083.

[39] A. E. f. t. P. A. Collaboration, "Science and detectors of the pierre auger observatory," arXiv:1004.2635 [astro-ph], Apr. 2010. arXiv: 1004.2635.

[40] D. Perkins, Particle Astrophysics. Oxford Master Series in Particle Physics, Astrophysics and Cosmology, Oxford University Press, 2nd edition ed., 2009.

[41] W. Heitler, The Quantum Theory of Radiation. Courier Dover Publications, 1954.

[42] H. Montanus, "An extended heitler-matthews model for the full hadronic cascade in cosmic air showers," arXiv:1311.0642 [astro-ph], Nov. 2013. arXiv: 1311.0642.

[43] B. akademiia na naukite, 15th International Cosmic Ray Conference : Conference Papers : Bulgarian Academy of Sciences, Plovdiv, Blgaria, August 13-26, 1977: EA session. 15th International Cosmic Ray Conference : Conference Papers : Bulgarian Academy of Sciences, Plovdiv, Blgaria, August 13-26, 1977, Bulgarian Academy of Sciences, 1977. 
[44] S. Kliewer, “Muons.” Berkeley, California USA: Lawrence Berkeley Laboratory. Retrieved 03-21-2014.

[45] K. J. C. J. N. S. G. T. T. Heck, D., "CORSIKA: A monte carlo code to simulate extensive air showers," Tech. Rep. FZKA6019, Forschunszentrum Karlsruhe, 1998.

[46] T. Bergmann, R. Engel, D. Heck, N. N. Kalmykov, S. Ostapchenko, T. Pierog, T. Thouw, and K. Werner, "One-dimensional hybrid approach to extensive air shower simulation,” Astroparticle Physics, vol. 26, pp. 420-432, Jan. 2007.

[47] N. N. Kalmykov, S. S. Ostapchenko, and A. I. Pavlov, "Quark-gluon-string model and EAS simulation problems at ultra-high energies," Nuclear Physics B - Proceedings Supplements, vol. 52, pp. 17-28, Feb. 1997.

[48] R. Engel, "Air shower calculations with the new version of SIBYLL," International Cosmic Ray Conference, vol. 1, p. 415, 1999.

[49] T. Pierog and K. Werner, "EPOS model and ultra high energy cosmic rays," Nuclear Physics B - Proceedings Supplements, vol. 196, pp. 102-105, Dec. 2009. arXiv: 0905.1198 .

[50] T. Pierog, I. Karpenko, S. Porteboeuf, and K. Werner, "New developments of EPOS 2," arXiv:1011.3748 [astro-ph, physics:hep-ph], Nov. 2010. arXiv: 1011.3748.

[51] R. D. Parsons, C. Bleve, S. S. Ostapchenko, and J. Knapp, "Systematic uncertainties in air shower measurements from high-energy hadronic interaction models," Astroparticle Physics, vol. 34, pp. 832-839, June 2011.

[52] P. A. Collaboration, "Measurement of the proton-air cross-section at $\$ \backslash \operatorname{sqrt}\{s\}=57 \$$ TeV with the pierre auger observatory," arXiv e-print 1208.1520, Aug. 2012. Phys. Rev. Lett. 109, 062002 (2012).

[53] The Pierre Auger Observatory, "Properties and performance of the prototype instrument for the Pierre Auger Observatory," Nuclear Instruments and Methods in Physics Research Section A: Accelerators, Spectrometers, Detectors and Associated Equipment, vol. 523, pp. 50-95, May 2004.

[54] the Pierre Auger Observatory, "Atmospheric monitoring for the auger fluorescence detector," 27th International Cosmic Ray Conference, pp. 745-748, 2001. 
[55] The Pierre Auger Observatory, "The fluorescence detector of the pierre auger observatory - a calorimeter for UHECR," arXiv e-print astro-ph/0608074, Aug. 2006. AIP Conf.Proc.867:175-182,2006.

[56] The Pierre Auger Collaboration, "The fluorescence detector of the pierre auger observatory," arXiv e-print 0907.4282, July 2009. Nucl.Instrum.Meth.A620:227251,2010 .

[57] the Pierre Auger Collaboration, "The fluorescence detector of the pierre auger observatory (CALOR2010 proceedings)," arXiv:1011.6523 [astro-ph], Nov. 2010. arXiv: 1011.6523 .

[58] T. P. A. Collaboration, "The exposure of the hybrid detector of the pierre auger observatory," Astroparticle Physics, vol. 34, pp. 368-381, Jan. 2011. arXiv: 1010.6162.

[59] the Pierre Auger Observatory, "Results from the Pierre Auger Observatory," Nuclear Instruments and Methods in Physics Research Section A: Accelerators, Spectrometers, Detectors and Associated Equipment, vol. 630, pp. 166-170, Feb. 2011.

[60] I. Allekotte, A. F. Barbosa, P. Bauleo, C. Bonifazi, B. Civit, C. O. Escobar, B. García, G. Guedes, M. Gómez Berisso, J. L. Harton, M. Healy, M. Kaducak, P. Mantsch, P. O. Mazur, C. Newman-Holmes, I. Pepe, I. Rodriguez-Cabo, H. Salazar, N. Smetniansky-De Grande, D. Warner, and for the Pierre Auger Collaboration, "The surface detector system of the Pierre Auger Observatory," Nuclear Instruments and Methods in Physics Research Section A: Accelerators, Spectrometers, Detectors and Associated Equipment, vol. 586, pp. 409-420, Mar. 2008. arXiv: 0712.2832.

[61] P. A. Collaboration, "Muons in air showers at the pierre auger observatory: Measurement of atmospheric production depth," Physical Review D, vol. 90, July 2014. arXiv: 1407.5919.

[62] L. Cazon, R. A. Vazquez, A. A. Watson, and E. Zas, "Time structure of muonic showers," Astroparticle Physics, vol. 21, pp. 71-86, Apr. 2004. arXiv: astro-ph/0311223.

[63] C. A. L. Bailer-Jones, M. Irwin, G. Gilmore, and T. von Hippel, "Physical parameterization of stellar spectra: The neural network approach," arXiv:astro-ph/9708206, Aug. 1997. arXiv: astro-ph/9708206.

[64] N. G. Phillips and A. Kogut, "Neural networks as a tool for parameter estimation in astrophysical data," arXiv:astro-ph/0112359, Dec. 2001. arXiv: astro-ph/0112359. 
[65] A. A. Collister and O. Lahav, "ANNz: estimating photometric redshifts using artificial neural networks," Publications of the Astronomical Society of the Pacific, vol. 116, pp. 345-351, Apr. 2004. arXiv: astro-ph/0311058.

[66] R. d'Abrusco, G. Longo, M. Paolillo, E. de Filippis, M. Brescia, A. Staiano, and R. Tagliaferri, "The use of neural networks to probe the structure of the nearby universe," arXiv:astro-ph/0701137, Jan. 2007. arXiv: astro-ph/0701137.

[67] S. Giridhar, A. Goswami, A. Kunder, S. Muneer, and G. S. Kumar, "The stellar parametrization using artificial neural network," arXiv:1203.2014 [astro-ph], Mar. 2012. arXiv: 1203.2014.

[68] B. Hoyle, M. M. Rau, R. Zitlau, S. Seitz, and J. Weller, "Feature importance for machine learning redshifts applied to SDSS galaxies," arXiv:1410.4696 [astro-ph], Oct. 2014. arXiv: 1410.4696.

[69] A. Gorecki, A. Abate, R. Ansari, A. Barrau, S. Baumont, M. Moniez, and J.-S. Ricol, "A new method to improve photometric redshift reconstruction. applications to the large synoptic survey telescope," arXiv:1301.3010 [astro-ph], Jan. 2013. arXiv: 1301.3010.

[70] J. Osborn, F. J. D. C. Juez, D. Guzman, A. Basden, T. J. Morris, E. Gendron, T. Butterley, R. M. Myers, A. Gueslaga, F. S. Lasheras, M. G. Victoria, M. L. S. Rodriguez, D. Gratadour, and G. Rousset, "Open-loop tomography with artificial neural networks on CANARY: on-sky results," Monthly Notices of the Royal Astronomical Society, vol. 441, pp. 2508-2514, May 2014. arXiv: 1405.6862.

[71] S. Bussino and S. M. Mari, "Gamma-hadron discrimination in extensive air showers using a neural network," Astroparticle Physics, vol. 15, pp. 65-77, Mar. 2001.

[72] P. Boinee, F. Barbarino, A. De Angelis, A. Saggion, and M. Zacchello, "Neural networks for gamma-hadron separation in MAGIC," arXiv:astro-ph/0503539, Mar. 2005. arXiv: astro-ph/0503539.

[73] M. Sharma, J. Nayak, M. K. Koul, S. Bose, and A. Mitra, "Gamma/hadron segregation for a ground based imaging atmospheric cherenkov telescope using machine learning methods: Random forest leads," arXiv:1410.5125 [astro-ph], Oct. 2014. arXiv: 1410.5125 . 
[74] A. K. O. Tiba, G. A. Medina-Tanco, and S. J. Sciutto, "Neural networks as a composition diagnostic for ultra-high energy cosmic rays," arXiv:astro-ph/0502255, Feb. 2005. arXiv: astro-ph/0502255.

[75] Z. Szadkowski and K. Pytel, "Artificial neural network as a FPGA trigger for a detection of very inclined air showers," arXiv:1406.1903 [astro-ph, physics:physics], June 2014. arXiv: 1406.1903.

[76] Z. Szadkowski, "Front-end board with cyclone $\mathrm{v}$ as a test high-resolution platform for the auger-beyond-2015 front end electronics," arXiv:1406.1918 [hep-ex, physics:physics], June 2014. arXiv: 1406.1918.

[77] R. Lippmann, "An introduction to computing with neural nets," IEEE ASSP Magazine, vol. 4, pp. 4-22, Apr. 1987.

[78] “ROOT data analysis framework user’s manual,” May 2014.

[79] P. K. F. Grieder, Extensive Air Showers: High Energy Phenomena and Astrophysical Aspects - A Tutorial, Reference Manual and Data Book. Springer, Aug. 2010.

[80] S. Riggi, A. Parra, G. Rodriguez, I. Valino, R. Vazquez, and E. Zas, "Identification of the primary mass of inclined cosmic ray showers from depth of maximum and number of muons parameters," arXiv e-print 1212.0218, Dec. 2012. Nuclear Instruments and Methods A, Volume 707, 11 April 2013, Pages 9-15.

[81] T. Antoni, W. Apel, A. Badea, K. Bekk, A. Bercuci, J. Blümer, H. Bozdog, I. Brancus, A. Chilingarian, K. Daumiller, P. Doll, R. Engel, J. Engler, F. Feßler, H. Gils, R. Glasstetter, A. Haungs, D. Heck, J. Hörandel, K.-H. Kampert, H. Klages, G. Maier, H. Mathes, H. Mayer, J. Milke, M. Müller, R. Obenland, J. Oehlschläger, S. Ostapchenko, M. Petcu, H. Rebel, A. Risse, M. Risse, M. Roth, G. Schatz, H. Schieler, J. Scholz, T. Thouw, H. Ulrich, J. van Buren, A. Vardanyan, A. Weindl, J. Wochele, and J. Zabierowski, "KASCADE measurements of energy spectra for elemental groups of cosmic rays: Results and open problems," Astroparticle Physics, vol. 24, pp. 1-25, Sept. 2005.

[82] A. Garyaka, R. Martirosov, S. Ter-Antonyan, N. Nikolskaya, Y. Gallant, L. Jones, and J. Procureur, "Rigidity-dependent cosmic ray energy spectra in the knee region obtained with the GAMMA experiment," Astroparticle Physics, vol. 28, pp. 169-181, Oct. 2007. 
[83] K.-H. Kampert and M. Unger, "Measurements of the cosmic ray composition with air shower experiments," Astroparticle Physics, vol. 35, pp. 660-678, May 2012.

[84] W. D. Apel, J. C. Arteaga, K. Bekk, M. Bertaina, J. Blümer, H. Bozdog, I. M. Brancus, P. Buchholz, C. Büttner, E. Cantoni, A. Chiavassa, F. Cossavella, K. Daumiller, V. de Souza, F. Di Pierro, P. Doll, R. Engel, J. Engler, M. Finger, D. Fuhrmann, P. L. Ghia, H. J. Gils, R. Glasstetter, C. Grupen, A. Haungs, D. Heck, J. R. Hörandel, T. Huege, P. G. Isar, K. H. Kampert, D. Kang, D. Kickelbick, H. O. Klages, K. Link, M. Ludwig, P. Łuczak, H. J. Mathes, H. J. Mayer, M. Melissas, J. Milke, B. Mitrica, C. Morello, G. Navarra, S. Nehls, R. Obenland, J. Oehlschläger, S. Ostapchenko, S. Over, N. Palmieri, M. Petcu, T. Pierog, H. Rebel, M. Roth, G. Schatz, H. Schieler, F. Schröder, O. Sima, G. Toma, G. C. Trinchero, H. Ulrich, A. Weindl, J. Wochele, M. Wommer, and J. Zabierowski, "Muon production height studies with the air shower experiment KASCADE-grande," Astroparticle Physics, vol. 34, pp. 476-485, Jan. 2011.

[85] S. Andringa, R. Conceição, and M. Pimenta, "Mass composition and cross-section from the shape of cosmic ray shower longitudinal profiles," Astroparticle Physics, vol. 34, pp. 360-367, Jan. 2011.

[86] CORSIKA School, Hybrid Simulations and CONEX, Nov 2008.

[87] B. Efron and R. J. Tibshirani, An Introduction to the Bootstrap. CRC Press, May 1994.

[88] A. E. Dolphin, "On the estimation of random uncertainties of star formation histories," arXiv:1308.1740 [astro-ph], Aug. 2013.

[89] L. Varga, P. Rakonczai, and A. Zempléni, "Applications of threshold models and the weighted bootstrap for hungarian precipitation data," arXiv:1310.7918 [math, stat], Oct. 2013.

[90] S. N. Lahiri, "Bootstrap methods: A review," in Frontiers in Statistics, pp. 231-255, Published bt Imperial College Press and Distributed by World Scientific Publishing Co., July 2006. 


\section{Appendices}

\section{Appendix A Results of pmANN+BPM}

\section{A.1 Results of pmANN+BPM for QGSJET}

Table A.1 : Calculated simulation and ANN values using pmANN+BPM for $\mathrm{p}$ in $\mathrm{p}-\mathrm{CNO}$ mixture of QGSJET showers at $\log (\mathrm{E} / \mathrm{eV})=18.75 .\left(\left\langle\mathrm{X}_{1}\right\rangle_{p}=42.46 \pm 1.39 \mathrm{~g} / \mathrm{cm}^{2}\right)$

\begin{tabular}{|c|c|c|c|c|}
\hline $\mathrm{f}$ & $\begin{array}{c}\text { Sim. Value } \\
{\left[\mathrm{g} / \mathrm{cm}^{2}\right]}\end{array}$ & $\begin{array}{c}\text { ANN Value } \\
{\left[\mathrm{g} / \mathrm{cm}^{2}\right]}\end{array}$ & $\begin{array}{c}\text { Sim. Difference } \\
{\left[\mathrm{g} / \mathrm{cm}^{2}\right]}\end{array}$ & $\begin{array}{c}\text { ANN Difference } \\
{\left[\mathrm{g} / \mathrm{cm}^{2}\right]}\end{array}$ \\
\hline \hline 0.10 & $21.14 \pm 1.21$ & $26.24 \pm 2.12$ & 21.32 & 16.22 \\
\hline 0.30 & $33.08 \pm 4.35$ & $36.79 \pm 4.15$ & 9.38 & 5.67 \\
\hline 0.50 & $36.54 \pm 4.27$ & $38.50 \pm 4.43$ & 5.92 & 3.96 \\
\hline 0.70 & $38.96 \pm 4.25$ & $39.28 \pm 4.71$ & 3.50 & 3.18 \\
\hline 0.90 & $40.88 \pm 4.08$ & $39.84 \pm 4.79$ & 1.58 & 2.62 \\
\hline
\end{tabular}

Table A.2 : Calculated simulation and ANN values using pmANN+BPM for CNO in p-CNO mixture of QGSJET showers at $\log (\mathrm{E} / \mathrm{eV})=18.75\left(\left\langle\mathrm{X}_{1}\right\rangle_{\mathrm{CNO}}=18.52 \pm 0.52 \mathrm{~g} / \mathrm{cm}^{2}\right)$

\begin{tabular}{|c|c|c|c|c|}
\hline $\mathrm{f}$ & $\begin{array}{c}\text { Sim. Value } \\
{\left[\mathrm{g} / \mathrm{cm}^{2}\right]}\end{array}$ & $\begin{array}{c}\text { ANN Value } \\
{\left[\mathrm{g} / \mathrm{cm}^{2}\right]}\end{array}$ & $\begin{array}{c}\text { Sim. Difference } \\
{\left[\mathrm{g} / \mathrm{cm}^{2}\right]}\end{array}$ & $\begin{array}{c}\text { ANN Difference } \\
{\left[\mathrm{g} / \mathrm{cm}^{2}\right]}\end{array}$ \\
\hline \hline 0.10 & $22.42 \pm 5.82$ & $16.05 \pm 1.71$ & -3.90 & 2.47 \\
\hline 0.30 & $17.87 \pm 3.66$ & $15.90 \pm 2.45$ & 0.65 & 2.62 \\
\hline 0.50 & $21.90 \pm 6.54$ & $15.71 \pm 2.97$ & -3.38 & 2.81 \\
\hline 0.70 & $20.60 \pm 4.70$ & $22.46 \pm 9.16$ & -2.08 & -3.94 \\
\hline 0.90 & $20.20 \pm 3.86$ & $28.98 \pm 3.08$ & -1.68 & -10.46 \\
\hline
\end{tabular}

Table A.3 : Calculated simulation and ANN values using pmANN+BPM for $\mathrm{p}$ in $\mathrm{p}-\mathrm{CNO}$ mixture of QGSJET showers at $\log (\mathrm{E} / \mathrm{eV})=19.00\left(\left\langle\mathrm{X}_{1}\right\rangle_{p}=40.35 \pm 1.26 \mathrm{~g} / \mathrm{cm}^{2}\right)$

\begin{tabular}{|c|c|c|c|c|}
\hline $\mathrm{f}$ & $\begin{array}{c}\text { Sim. Value } \\
{\left[\mathrm{g} / \mathrm{cm}^{2}\right]}\end{array}$ & $\begin{array}{c}\text { ANN Value } \\
{\left[\mathrm{g} / \mathrm{cm}^{2}\right]}\end{array}$ & $\begin{array}{c}\text { Sim. Difference } \\
{\left[\mathrm{g} / \mathrm{cm}^{2}\right]}\end{array}$ & $\begin{array}{c}\text { ANN Difference } \\
{\left[\mathrm{g} / \mathrm{cm}^{2}\right]}\end{array}$ \\
\hline \hline 0.10 & $21.25 \pm 1.45$ & $24.22 \pm 2.13$ & 19.10 & 16.13 \\
\hline 0.30 & $29.39 \pm 4.65$ & $36.80 \pm 5.10$ & 10.96 & 3.55 \\
\hline 0.50 & $31.85 \pm 4.71$ & $38.01 \pm 4.77$ & 8.50 & 2.34 \\
\hline 0.70 & $34.19 \pm 4.75$ & $38.30 \pm 4.69$ & 6.16 & 2.05 \\
\hline 0.90 & $36.19 \pm 4.75$ & $37.92 \pm 4.30$ & 4.16 & 2.43 \\
\hline
\end{tabular}


Table A.4 : Calculated simulation and ANN values using pmANN+BPM for CNO in p-CNO mixture of QGSJET showers at $\log (\mathrm{E} / \mathrm{eV})=19.00\left(\left\langle\mathrm{X}_{1}\right\rangle_{C N O}=18.20 \pm 0.48 \mathrm{~g} / \mathrm{cm}^{2}\right)$

\begin{tabular}{|c|c|c|c|c|}
\hline $\mathrm{f}$ & $\begin{array}{c}\text { Sim. Value } \\
{\left[\mathrm{g} / \mathrm{cm}^{2}\right]}\end{array}$ & $\begin{array}{c}\text { ANN Value } \\
{\left[\mathrm{g} / \mathrm{cm}^{2}\right]}\end{array}$ & $\begin{array}{c}\text { Sim. Difference } \\
{\left[\mathrm{g} / \mathrm{cm}^{2}\right]}\end{array}$ & $\begin{array}{c}\text { ANN Difference } \\
{\left[\mathrm{g} / \mathrm{cm}^{2}\right]}\end{array}$ \\
\hline \hline 0.10 & $21.39 \pm 6.13$ & $15.49 \pm 2.24$ & -3.19 & 2.71 \\
\hline 0.30 & $19.41 \pm 5.56$ & $16.56 \pm 2.38$ & -1.21 & 1.64 \\
\hline 0.50 & $18.81 \pm 5.58$ & $16.32 \pm 3.01$ & -0.61 & 1.88 \\
\hline 0.70 & $23.13 \pm 5.82$ & $16.54 \pm 7.28$ & -4.93 & 1.66 \\
\hline 0.90 & $22.06 \pm 4.35$ & $29.11 \pm 3.30$ & -3.86 & -10.91 \\
\hline
\end{tabular}

Table A.5 : Calculated simulation and ANN values using pmANN+BPM for $\mathrm{p}$ in $\mathrm{p}-\mathrm{CNO}$ mixture of QGSJET showers at $\log (\mathrm{E} / \mathrm{eV})=19.50\left(\left\langle\mathrm{X}_{1}\right\rangle_{p}=41.96 \pm 1.40 \mathrm{~g} / \mathrm{cm}^{2}\right)$

\begin{tabular}{|c|c|c|c|c|}
\hline $\mathrm{f}$ & $\begin{array}{c}\text { Sim. Value } \\
{\left[\mathrm{g} / \mathrm{cm}^{2}\right]}\end{array}$ & $\begin{array}{c}\text { ANN Value } \\
{\left[\mathrm{g} / \mathrm{cm}^{2}\right]}\end{array}$ & $\begin{array}{c}\text { Sim. Difference } \\
{\left[\mathrm{g} / \mathrm{cm}^{2}\right]}\end{array}$ & $\begin{array}{c}\text { ANN Difference } \\
{\left[\mathrm{g} / \mathrm{cm}^{2}\right]}\end{array}$ \\
\hline \hline 0.10 & $20.60 \pm 1.94$ & $21.70 \pm 2.34$ & 21.36 & 20.26 \\
\hline 0.30 & $31.10 \pm 6.31$ & $44.14 \pm 8.61$ & 10.86 & -2.18 \\
\hline 0.50 & $31.12 \pm 5.29$ & $46.51 \pm 8.19$ & 10.84 & -4.55 \\
\hline 0.70 & $31.82 \pm 4.98$ & $47.25 \pm 8.00$ & 10.14 & -5.29 \\
\hline 0.90 & $33.00 \pm 4.73$ & $47.39 \pm 7.92$ & 8.96 & -5.43 \\
\hline
\end{tabular}

Table A.6 : Calculated simulation and ANN values using pmANN+BPM for CNO in p-CNO mixture of QGSJET showers at $\log (\mathrm{E} / \mathrm{eV})=19.50\left(\left\langle\mathrm{X}_{1}\right\rangle_{C N O}=17.47 \pm 0.46 \mathrm{~g} / \mathrm{cm}^{2}\right)$

\begin{tabular}{|c|c|c|c|c|}
\hline $\mathrm{f}$ & $\begin{array}{c}\text { Sim. Value } \\
{\left[\mathrm{g} / \mathrm{cm}^{2}\right]}\end{array}$ & $\begin{array}{c}\text { ANN Value } \\
{\left[\mathrm{g} / \mathrm{cm}^{2}\right]}\end{array}$ & $\begin{array}{c}\text { Sim. Difference } \\
{\left[\mathrm{g} / \mathrm{cm}^{2}\right]}\end{array}$ & $\begin{array}{c}\text { ANN Difference } \\
{\left[\mathrm{g} / \mathrm{cm}^{2}\right]}\end{array}$ \\
\hline \hline 0.10 & $18.94 \pm 6.57$ & $16.60 \pm 4.02$ & -1.47 & 0.87 \\
\hline 0.30 & $17.41 \pm 5.34$ & $17.56 \pm 1.71$ & 0.06 & -0.09 \\
\hline 0.50 & $18.43 \pm 4.74$ & $17.89 \pm 2.59$ & -0.96 & -0.42 \\
\hline 0.70 & $22.34 \pm 5.59$ & $18.79 \pm 5.11$ & -4.87 & -1.32 \\
\hline 0.90 & $22.16 \pm 5.16$ & $21.32 \pm 5.94$ & -4.69 & -3.85 \\
\hline
\end{tabular}


Table A.7 : Calculated simulation and ANN values using pmANN+BPM for $\mathrm{p}$ in $\mathrm{p}-\mathrm{CNO}$ mixture of QGSJET showers at $\log (\mathrm{E} / \mathrm{eV})=20.00\left(\left\langle\mathrm{X}_{1}\right\rangle_{p}=39.60 \pm 1.32 \mathrm{~g} / \mathrm{cm}^{2}\right)$

\begin{tabular}{|c|c|c|c|c|}
\hline $\mathrm{f}$ & $\begin{array}{c}\text { Sim. Value } \\
{\left[\mathrm{g} / \mathrm{cm}^{2}\right]}\end{array}$ & $\begin{array}{c}\text { ANN Value } \\
{\left[\mathrm{g} / \mathrm{cm}^{2}\right]}\end{array}$ & $\begin{array}{c}\text { Sim. Difference } \\
{\left[\mathrm{g} / \mathrm{cm}^{2}\right]}\end{array}$ & $\begin{array}{c}\text { ANN Difference } \\
{\left[\mathrm{g} / \mathrm{cm}^{2}\right]}\end{array}$ \\
\hline \hline 0.10 & $20.58 \pm 2.04$ & $19.91 \pm 2.26$ & 19.02 & 19.69 \\
\hline 0.30 & $34.83 \pm 8.30$ & $44.42 \pm 9.73$ & 4.77 & -4.82 \\
\hline 0.50 & $35.30 \pm 7.01$ & $46.76 \pm 8.81$ & 4.30 & -7.16 \\
\hline 0.70 & $35.94 \pm 6.62$ & $48.00 \pm 8.65$ & 3.66 & -8.40 \\
\hline 0.90 & $37.39 \pm 6.58$ & $48.12 \pm 8.21$ & 2.21 & -8.52 \\
\hline
\end{tabular}

Table A.8 : Calculated simulation and ANN values using pmANN+BPM for CNO in p-CNO mixture of QGSJET showers at $\log (\mathrm{E} / \mathrm{eV})=20.00\left(\left\langle\mathrm{X}_{1}\right\rangle_{C N O}=16.90 \pm 0.46 \mathrm{~g} / \mathrm{cm}^{2}\right)$

\begin{tabular}{|c|c|c|c|c|}
\hline $\mathrm{f}$ & $\begin{array}{c}\text { Sim. Value } \\
{\left[\mathrm{g} / \mathrm{cm}^{2}\right]}\end{array}$ & $\begin{array}{c}\text { ANN Value } \\
{\left[\mathrm{g} / \mathrm{cm}^{2}\right]}\end{array}$ & $\begin{array}{c}\text { Sim. Difference } \\
{\left[\mathrm{g} / \mathrm{cm}^{2}\right]}\end{array}$ & $\begin{array}{c}\text { ANN Difference } \\
{\left[\mathrm{g} / \mathrm{cm}^{2}\right]}\end{array}$ \\
\hline \hline 0.10 & $16.64 \pm 7.04$ & $18.96 \pm 7.80$ & 0.26 & -2.06 \\
\hline 0.30 & $15.68 \pm 4.55$ & $19.06 \pm 1.70$ & 1.22 & -2.16 \\
\hline 0.50 & $16.68 \pm 4.88$ & $19.53 \pm 2.42$ & 0.22 & -2.63 \\
\hline 0.70 & $20.03 \pm 6.41$ & $20.16 \pm 4.54$ & -3.13 & -3.26 \\
\hline 0.90 & $22.36 \pm 4.91$ & $21.60 \pm 5.50$ & -5.46 & -4.70 \\
\hline
\end{tabular}

Table A.9 : Calculated simulation and ANN values using pmANN+BPM for $\mathrm{p}$ in $\mathrm{p}$-iron mixture of QGSJET showers at $\log (\mathrm{E} / \mathrm{eV})=18.75\left(\left\langle\mathrm{X}_{1}\right\rangle_{p}=42.46 \pm 1.39 \mathrm{~g} / \mathrm{cm}^{2}\right)$

\begin{tabular}{|c|c|c|c|c|}
\hline $\mathrm{f}$ & $\begin{array}{c}\text { Sim. Value } \\
{\left[\mathrm{g} / \mathrm{cm}^{2}\right]}\end{array}$ & $\begin{array}{c}\text { ANN Value } \\
{\left[\mathrm{g} / \mathrm{cm}^{2}\right]}\end{array}$ & $\begin{array}{c}\text { Sim. Difference } \\
{\left[\mathrm{g} / \mathrm{cm}^{2}\right]}\end{array}$ & $\begin{array}{c}\text { ANN Difference } \\
{\left[\mathrm{g} / \mathrm{cm}^{2}\right]}\end{array}$ \\
\hline \hline 0.10 & $19.99 \pm 2.46$ & $21.89 \pm 2.66$ & 22.47 & 20.57 \\
\hline 0.30 & $38.29 \pm 7.49$ & $42.40 \pm 6.97$ & 4.17 & 0.06 \\
\hline 0.50 & $40.14 \pm 5.36$ & $42.16 \pm 5.64$ & 2.32 & 0.30 \\
\hline 0.70 & $40.90 \pm 4.86$ & $40.80 \pm 5.13$ & 1.56 & 1.66 \\
\hline 0.90 & $41.54 \pm 4.34$ & $40.23 \pm 4.95$ & 0.92 & 2.23 \\
\hline
\end{tabular}

Table A.10 : Calculated simulation and ANN values using pmANN+BPM for iron in p-iron mixture of QGSJET showers at $\log (\mathrm{E} / \mathrm{eV})=18.75\left(\left\langle\mathrm{X}_{1}\right\rangle_{F e}=10.39 \pm 0.59 \mathrm{~g} / \mathrm{cm}^{2}\right)$

\begin{tabular}{|c|c|c|c|c|}
\hline $\mathrm{f}$ & $\begin{array}{c}\text { Sim. Value } \\
{\left[\mathrm{g} / \mathrm{cm}^{2}\right]}\end{array}$ & $\begin{array}{c}\text { ANN Value } \\
{\left[\mathrm{g} / \mathrm{cm}^{2}\right]}\end{array}$ & $\begin{array}{c}\text { Sim. Difference } \\
{\left[\mathrm{g} / \mathrm{cm}^{2}\right]}\end{array}$ & $\begin{array}{c}\text { ANN Difference } \\
{\left[\mathrm{g} / \mathrm{cm}^{2}\right]}\end{array}$ \\
\hline \hline 0.10 & $7.78 \pm 2.36$ & $8.04 \pm 1.00$ & 2.61 & 2.35 \\
\hline 0.30 & $13.57 \pm 4.43$ & $9.44 \pm 1.33$ & -3.18 & 0.95 \\
\hline 0.50 & $19.15 \pm 7.31$ & $8.61 \pm 1.75$ & -8.76 & 1.78 \\
\hline 0.70 & $21.31 \pm 4.08$ & $8.02 \pm 2.77$ & -10.92 & 2.37 \\
\hline 0.90 & $21.08 \pm 4.08$ & $25.20 \pm 3.39$ & -10.69 & -14.81 \\
\hline
\end{tabular}


Table A.11 : Calculated simulation and ANN values using pmANN+BPM for $\mathrm{p}$ in $\mathrm{p}$-iron mixture of QGSJET showers at $\log (\mathrm{E} / \mathrm{eV})=19.00\left(\left\langle\mathrm{X}_{1}\right\rangle_{p}=40.35 \pm 1.26 \mathrm{~g} / \mathrm{cm}^{2}\right)$

\begin{tabular}{|c|c|c|c|c|}
\hline $\mathrm{f}$ & $\begin{array}{c}\text { Sim. Value } \\
{\left[\mathrm{g} / \mathrm{cm}^{2}\right]}\end{array}$ & $\begin{array}{c}\text { ANN Value } \\
{\left[\mathrm{g} / \mathrm{cm}^{2}\right]}\end{array}$ & $\begin{array}{c}\text { Sim. Difference } \\
{\left[\mathrm{g} / \mathrm{cm}^{2}\right]}\end{array}$ & $\begin{array}{c}\text { ANN Difference } \\
{\left[\mathrm{g} / \mathrm{cm}^{2}\right]}\end{array}$ \\
\hline \hline 0.10 & $19.60 \pm 3.09$ & $19.70 \pm 2.85$ & 20.75 & 20.65 \\
\hline 0.30 & $33.74 \pm 7.12$ & $38.84 \pm 8.14$ & 6.61 & 1.51 \\
\hline 0.50 & $35.58 \pm 5.67$ & $42.09 \pm 5.89$ & 4.77 & -1.74 \\
\hline 0.70 & $35.94 \pm 4.87$ & $39.77 \pm 4.89$ & 4.41 & 0.58 \\
\hline 0.90 & $36.54 \pm 4.40$ & $38.38 \pm 4.38$ & 3.81 & 1.97 \\
\hline
\end{tabular}

Table A.12 : Calculated simulation and ANN values using pmANN+BPM for iron in p-iron mixture of QGSJET showers at $\log (\mathrm{E} / \mathrm{eV})=19.00\left(\left\langle\mathrm{X}_{1}\right\rangle_{\mathrm{Fe}}=10.36 \pm 0.60 \mathrm{~g} / \mathrm{cm}^{2}\right)$

\begin{tabular}{|c|c|c|c|c|}
\hline $\mathrm{f}$ & $\begin{array}{c}\text { Sim. Value } \\
{\left[\mathrm{g} / \mathrm{cm}^{2}\right]}\end{array}$ & $\begin{array}{c}\text { ANN Value } \\
{\left[\mathrm{g} / \mathrm{cm}^{2}\right]}\end{array}$ & $\begin{array}{c}\text { Sim. Difference } \\
{\left[\mathrm{g} / \mathrm{cm}^{2}\right]}\end{array}$ & $\begin{array}{c}\text { ANN Difference } \\
{\left[\mathrm{g} / \mathrm{cm}^{2}\right]}\end{array}$ \\
\hline \hline 0.10 & $7.86 \pm 2.78$ & $8.07 \pm 1.05$ & 2.50 & 2.29 \\
\hline 0.30 & $13.02 \pm 5.24$ & $9.07 \pm 1.34$ & -2.66 & 1.29 \\
\hline 0.50 & $17.05 \pm 7.38$ & $9.12 \pm 1.77$ & -6.69 & 1.24 \\
\hline 0.70 & $23.96 \pm 4.72$ & $8.18 \pm 2.64$ & -13.60 & 2.18 \\
\hline 0.90 & $21.88 \pm 4.15$ & $25.78 \pm 3.39$ & -11.52 & -15.42 \\
\hline
\end{tabular}

Table A.13 : Calculated simulation and ANN values using pmANN+BPM for $\mathrm{p}$ in p-iron mixture of QGSJET showers at $\log (\mathrm{E} / \mathrm{eV})=19.50\left(\left\langle\mathrm{X}_{1}\right\rangle_{p}=41.96 \pm 1.40 \mathrm{~g} / \mathrm{cm}^{2}\right)$

\begin{tabular}{|c|c|c|c|c|}
\hline $\mathrm{f}$ & $\begin{array}{c}\text { Sim. Value } \\
{\left[\mathrm{g} / \mathrm{cm}^{2}\right]}\end{array}$ & $\begin{array}{c}\text { ANN Value } \\
{\left[\mathrm{g} / \mathrm{cm}^{2}\right]}\end{array}$ & $\begin{array}{c}\text { Sim. Difference } \\
{\left[\mathrm{g} / \mathrm{cm}^{2}\right]}\end{array}$ & $\begin{array}{c}\text { ANN Difference } \\
{\left[\mathrm{g} / \mathrm{cm}^{2}\right]}\end{array}$ \\
\hline \hline 0.10 & $20.86 \pm 4.57$ & $17.87 \pm 2.59$ & 21.10 & 24.09 \\
\hline 0.30 & $35.98 \pm 8.95$ & $39.90 \pm 13.03$ & 5.98 & 2.06 \\
\hline 0.50 & $35.33 \pm 6.81$ & $50.18 \pm 8.63$ & 6.63 & -8.22 \\
\hline 0.70 & $34.16 \pm 5.46$ & $49.03 \pm 8.26$ & 7.80 & -7.07 \\
\hline 0.90 & $34.42 \pm 4.93$ & $47.91 \pm 8.06$ & 7.54 & -5.95 \\
\hline
\end{tabular}

Table A.14 : Calculated simulation and ANN values using pmANN+BPM for iron in p-iron mixture of QGSJET showers at $\log (\mathrm{E} / \mathrm{eV})=19.50\left(\left\langle\mathrm{X}_{1}\right\rangle_{F e}=10.52 \pm 0.64 \mathrm{~g} / \mathrm{cm}^{2}\right)$

\begin{tabular}{|c|c|c|c|c|}
\hline $\mathrm{f}$ & $\begin{array}{c}\text { Sim. Value } \\
{\left[\mathrm{g} / \mathrm{cm}^{2}\right]}\end{array}$ & $\begin{array}{c}\text { ANN Value } \\
{\left[\mathrm{g} / \mathrm{cm}^{2}\right]}\end{array}$ & $\begin{array}{c}\text { Sim. Difference } \\
{\left[\mathrm{g} / \mathrm{cm}^{2}\right]}\end{array}$ & $\begin{array}{c}\text { ANN Difference } \\
{\left[\mathrm{g} / \mathrm{cm}^{2}\right]}\end{array}$ \\
\hline \hline 0.10 & $7.85 \pm 2.08$ & $8.26 \pm 1.14$ & 2.67 & 2.26 \\
\hline 0.30 & $13.12 \pm 4.96$ & $10.02 \pm 1.75$ & -2.60 & 0.50 \\
\hline 0.50 & $18.13 \pm 6.27$ & $11.35 \pm 2.06$ & -7.61 & -0.83 \\
\hline 0.70 & $23.67 \pm 4.89$ & $11.40 \pm 3.15$ & -13.15 & -0.88 \\
\hline 0.90 & $21.96 \pm 4.99$ & $16.68 \pm 6.46$ & -11.44 & -6.16 \\
\hline
\end{tabular}


Table A.15 : Calculated simulation and ANN values using pmANN+BPM for $\mathrm{p}$ in $\mathrm{p}$-iron mixture of QGSJET showers at $\log (\mathrm{E} / \mathrm{eV})=20.00\left(\left\langle\mathrm{X}_{1}\right\rangle_{p}=39.60 \pm 1.32 \mathrm{~g} / \mathrm{cm}^{2}\right)$

\begin{tabular}{|c|c|c|c|c|}
\hline $\mathrm{f}$ & $\begin{array}{c}\text { Sim. Value } \\
{\left[\mathrm{g} / \mathrm{cm}^{2}\right]}\end{array}$ & $\begin{array}{c}\text { ANN Value } \\
{\left[\mathrm{g} / \mathrm{cm}^{2}\right]}\end{array}$ & $\begin{array}{c}\text { Sim. Difference } \\
{\left[\mathrm{g} / \mathrm{cm}^{2}\right]}\end{array}$ & $\begin{array}{c}\text { ANN Difference } \\
{\left[\mathrm{g} / \mathrm{cm}^{2}\right]}\end{array}$ \\
\hline \hline 0.10 & $21.86 \pm 6.14$ & $15.22 \pm 2.74$ & 17.74 & 24.38 \\
\hline 0.30 & $37.90 \pm 11.18$ & $36.88 \pm 13.68$ & 1.70 & 2.72 \\
\hline 0.50 & $42.03 \pm 8.32$ & $49.87 \pm 8.53$ & -2.43 & -10.27 \\
\hline 0.70 & $39.42 \pm 7.00$ & $48.48 \pm 8.20$ & 0.18 & -8.88 \\
\hline 0.90 & $38.90 \pm 5.98$ & $46.72 \pm 7.97$ & 0.70 & -7.12 \\
\hline
\end{tabular}

Table A.16 : Calculated simulation and ANN values using pmANN+BPM for iron in p-iron mixture of QGSJET showers at $\log (\mathrm{E} / \mathrm{eV})=20.00\left(\left\langle\mathrm{X}_{1}\right\rangle_{F e}=9.75 \pm 0.58 \mathrm{~g} / \mathrm{cm}^{2}\right)$

\begin{tabular}{|c|c|c|c|c|}
\hline $\mathrm{f}$ & $\begin{array}{c}\text { Sim. Value } \\
{\left[\mathrm{g} / \mathrm{cm}^{2}\right]}\end{array}$ & $\begin{array}{c}\text { ANN Value } \\
{\left[\mathrm{g} / \mathrm{cm}^{2}\right]}\end{array}$ & $\begin{array}{c}\text { Sim. Difference } \\
{\left[\mathrm{g} / \mathrm{cm}^{2}\right]}\end{array}$ & $\begin{array}{c}\text { ANN Difference } \\
{\left[\mathrm{g} / \mathrm{cm}^{2}\right]}\end{array}$ \\
\hline \hline 0.10 & $7.53 \pm 1.13$ & $9.29 \pm 1.37$ & 2.22 & 0.46 \\
\hline 0.30 & $11.77 \pm 4.40$ & $10.22 \pm 1.63$ & -2.02 & -0.47 \\
\hline 0.50 & $14.99 \pm 5.17$ & $11.75 \pm 1.82$ & -5.24 & -2.00 \\
\hline 0.70 & $20.38 \pm 6.02$ & $11.80 \pm 2.86$ & -10.63 & -2.05 \\
\hline 0.90 & $21.61 \pm 4.72$ & $16.46 \pm 5.71$ & -11.86 & -6.71 \\
\hline
\end{tabular}

Table A.17 : Calculated simulation and ANN values using pmANN+BPM for CNO in CNO-iron mixture of QGSJET showers at $\log (\mathrm{E} / \mathrm{eV})=18.75\left(\left\langle\mathrm{X}_{1}\right\rangle_{C N O}=18.52 \pm 0.52 \mathrm{~g} / \mathrm{cm}^{2}\right)$

\begin{tabular}{|c|c|c|c|c|}
\hline $\mathrm{f}$ & $\begin{array}{c}\text { Sim. Value } \\
{\left[\mathrm{g} / \mathrm{cm}^{2}\right]}\end{array}$ & $\begin{array}{c}\text { ANN Value } \\
{\left[\mathrm{g} / \mathrm{cm}^{2}\right]}\end{array}$ & $\begin{array}{c}\text { Sim. Difference } \\
{\left[\mathrm{g} / \mathrm{cm}^{2}\right]}\end{array}$ & $\begin{array}{c}\text { ANN Difference } \\
{\left[\mathrm{g} / \mathrm{cm}^{2}\right]}\end{array}$ \\
\hline \hline 0.10 & $12.11 \pm 1.34$ & $15.35 \pm 1.16$ & 6.41 & 3.17 \\
\hline 0.30 & $14.31 \pm 1.30$ & $17.54 \pm 1.17$ & 4.21 & 0.98 \\
\hline 0.50 & $15.71 \pm 1.23$ & $19.18 \pm 1.14$ & 2.81 & -0.66 \\
\hline 0.70 & $16.61 \pm 1.18$ & $20.37 \pm 1.14$ & 1.91 & -1.85 \\
\hline 0.90 & $17.33 \pm 1.07$ & $21.30 \pm 1.13$ & 1.19 & -2.78 \\
\hline
\end{tabular}

Table A.18 : Calculated simulation and ANN values using pmANN+BPM for iron in CNO-iron mixture of QGSJET showers at $\log (\mathrm{E} / \mathrm{eV})=18.75\left(\left\langle\mathrm{X}_{1}\right\rangle_{F e}=10.39 \pm 0.59 \mathrm{~g} / \mathrm{cm}^{2}\right)$

\begin{tabular}{|c|c|c|c|c|}
\hline $\mathrm{f}$ & $\begin{array}{c}\text { Sim. Value } \\
{\left[\mathrm{g} / \mathrm{cm}^{2}\right]}\end{array}$ & $\begin{array}{c}\text { ANN Value } \\
{\left[\mathrm{g} / \mathrm{cm}^{2}\right]}\end{array}$ & $\begin{array}{c}\text { Sim. Difference } \\
{\left[\mathrm{g} / \mathrm{cm}^{2}\right]}\end{array}$ & $\begin{array}{c}\text { ANN Difference } \\
{\left[\mathrm{g} / \mathrm{cm}^{2}\right]}\end{array}$ \\
\hline \hline 0.10 & $12.48 \pm 6.20$ & $10.44 \pm 4.21$ & -2.09 & -0.05 \\
\hline 0.30 & $15.32 \pm 6.16$ & $10.07 \pm 3.27$ & -4.93 & 0.32 \\
\hline 0.50 & $18.73 \pm 6.03$ & $10.23 \pm 2.99$ & -8.34 & 0.16 \\
\hline 0.70 & $19.09 \pm 5.64$ & $10.43 \pm 2.45$ & -8.70 & -0.04 \\
\hline 0.90 & $20.35 \pm 7.49$ & $11.78 \pm 2.49$ & -9.96 & -1.39 \\
\hline
\end{tabular}


Table A.19 : Calculated simulation and ANN values using pmANN+BPM for CNO in CNO-iron mixture of QGSJET showers at $\log (\mathrm{E} / \mathrm{eV})=19.00\left(\left\langle\mathrm{X}_{1}\right\rangle_{C N O}=18.20 \pm 0.48 \mathrm{~g} / \mathrm{cm}^{2}\right)$

\begin{tabular}{|c|c|c|c|c|}
\hline $\mathrm{f}$ & $\begin{array}{c}\text { Sim. Value } \\
{\left[\mathrm{g} / \mathrm{cm}^{2}\right]}\end{array}$ & $\begin{array}{c}\text { ANN Value } \\
{\left[\mathrm{g} / \mathrm{cm}^{2}\right]}\end{array}$ & $\begin{array}{c}\text { Sim. Difference } \\
{\left[\mathrm{g} / \mathrm{cm}^{2}\right]}\end{array}$ & $\begin{array}{c}\text { ANN Difference } \\
{\left[\mathrm{g} / \mathrm{cm}^{2}\right]}\end{array}$ \\
\hline \hline 0.10 & $11.88 \pm 1.64$ & $14.49 \pm 1.31$ & 6.32 & 3.71 \\
\hline 0.30 & $13.98 \pm 1.58$ & $16.75 \pm 1.34$ & 4.22 & 1.45 \\
\hline 0.50 & $15.52 \pm 1.54$ & $18.43 \pm 1.31$ & 2.68 & -0.23 \\
\hline 0.70 & $16.67 \pm 1.50$ & $19.66 \pm 1.28$ & 1.53 & -1.46 \\
\hline 0.90 & $17.55 \pm 1.40$ & $20.58 \pm 1.25$ & 0.65 & -2.38 \\
\hline
\end{tabular}

Table A.20 : Calculated simulation and ANN values using pmANN+BPM for iron in CNO-iron mixture of QGSJET showers at $\log (\mathrm{E} / \mathrm{eV})=19.00\left(\left\langle\mathrm{X}_{1}\right\rangle_{\mathrm{Fe}}=10.36 \pm 0.60 \mathrm{~g} / \mathrm{cm}^{2}\right)$

\begin{tabular}{|c|c|c|c|c|}
\hline $\mathrm{f}$ & $\begin{array}{c}\text { Sim. Value } \\
{\left[\mathrm{g} / \mathrm{cm}^{2}\right]}\end{array}$ & $\begin{array}{c}\text { ANN Value } \\
{\left[\mathrm{g} / \mathrm{cm}^{2}\right]}\end{array}$ & $\begin{array}{c}\text { Sim. Difference } \\
{\left[\mathrm{g} / \mathrm{cm}^{2}\right]}\end{array}$ & $\begin{array}{c}\text { ANN Difference } \\
{\left[\mathrm{g} / \mathrm{cm}^{2}\right]}\end{array}$ \\
\hline \hline 0.10 & $11.53 \pm 6.35$ & $10.32 \pm 5.19$ & -1.17 & 0.04 \\
\hline 0.30 & $14.80 \pm 6.78$ & $10.61 \pm 4.32$ & -4.44 & -0.25 \\
\hline 0.50 & $16.89 \pm 6.68$ & $11.05 \pm 4.58$ & -6.53 & -0.69 \\
\hline 0.70 & $18.29 \pm 5.90$ & $11.33 \pm 3.41$ & -7.93 & -0.97 \\
\hline 0.90 & $20.31 \pm 6.92$ & $12.97 \pm 4.65$ & -9.95 & -2.61 \\
\hline
\end{tabular}

Table A.21 : Calculated simulation and ANN values using pmANN+BPM for CNO in CNO-iron mixture of QGSJET showers at $\log (\mathrm{E} / \mathrm{eV})=19.50\left(\left\langle\mathrm{X}_{1}\right\rangle_{C N O}=17.47 \pm 0.46 \mathrm{~g} / \mathrm{cm}^{2}\right)$

\begin{tabular}{|c|c|c|c|c|}
\hline $\mathrm{f}$ & $\begin{array}{c}\text { Sim. Value } \\
{\left[\mathrm{g} / \mathrm{cm}^{2}\right]}\end{array}$ & $\begin{array}{c}\text { ANN Value } \\
{\left[\mathrm{g} / \mathrm{cm}^{2}\right]}\end{array}$ & $\begin{array}{c}\text { Sim. Difference } \\
{\left[\mathrm{g} / \mathrm{cm}^{2}\right]}\end{array}$ & $\begin{array}{c}\text { ANN Difference } \\
{\left[\mathrm{g} / \mathrm{cm}^{2}\right]}\end{array}$ \\
\hline \hline 0.10 & $12.72 \pm 2.42$ & $14.04 \pm 1.33$ & 4.75 & 3.43 \\
\hline 0.30 & $14.31 \pm 2.08$ & $15.56 \pm 1.40$ & 3.16 & 1.91 \\
\hline 0.50 & $15.28 \pm 1.95$ & $16.83 \pm 1.38$ & 2.19 & 0.64 \\
\hline 0.70 & $16.00 \pm 1.85$ & $17.76 \pm 1.38$ & 1.47 & -0.29 \\
\hline 0.90 & $16.64 \pm 1.76$ & $18.53 \pm 1.42$ & 0.83 & -1.06 \\
\hline
\end{tabular}


Table A.22 : Calculated simulation and ANN values using pmANN+BPM for iron in CNO-iron mixture of QGSJET showers at $\log (\mathrm{E} / \mathrm{eV})=19.50\left(\left\langle\mathrm{X}_{1}\right\rangle_{F e}=10.52 \pm 0.64 \mathrm{~g} / \mathrm{cm}^{2}\right)$

\begin{tabular}{|c|c|c|c|c|}
\hline $\mathrm{f}$ & $\begin{array}{c}\text { Sim. Value } \\
{\left[\mathrm{g} / \mathrm{cm}^{2}\right]}\end{array}$ & $\begin{array}{c}\text { ANN Value } \\
{\left[\mathrm{g} / \mathrm{cm}^{2}\right]}\end{array}$ & $\begin{array}{c}\text { Sim. Difference } \\
{\left[\mathrm{g} / \mathrm{cm}^{2}\right]}\end{array}$ & $\begin{array}{c}\text { ANN Difference } \\
{\left[\mathrm{g} / \mathrm{cm}^{2}\right]}\end{array}$ \\
\hline \hline 0.10 & $9.60 \pm 5.46$ & $10.41 \pm 5.27$ & 0.92 & 0.11 \\
\hline 0.30 & $12.44 \pm 6.42$ & $11.65 \pm 4.87$ & -1.92 & -1.13 \\
\hline 0.50 & $15.23 \pm 6.96$ & $13.45 \pm 6.20$ & -4.71 & -2.93 \\
\hline 0.70 & $16.31 \pm 6.46$ & $13.86 \pm 4.74$ & -5.79 & -3.34 \\
\hline 0.90 & $17.37 \pm 6.22$ & $15.61 \pm 7.14$ & -6.85 & -5.09 \\
\hline
\end{tabular}

Table A.23 : Calculated simulation and ANN values using pmANN+BPM for $\mathrm{CNO}$ in CNO-iron mixture of QGSJET showers at $\log (\mathrm{E} / \mathrm{eV})=20.00$ $\left(\left\langle\mathrm{X}_{1}\right\rangle_{C N O}=16.90 \pm 0.46 \mathrm{~g} / \mathrm{cm}^{2}\right)$

Calculated simulation and ANN values using pmANN+BPM for CNO in CNO-iron mixture of QGSJET showers at $\log (\mathrm{E} / \mathrm{eV})=20.00\left(\left\langle\mathrm{X}_{1}\right\rangle_{C N O}=16.90 \pm 0.46 \mathrm{~g} / \mathrm{cm}^{2}\right)$

\begin{tabular}{|c|c|c|c|c|}
\hline $\mathrm{f}$ & $\begin{array}{c}\text { Sim. Value } \\
{\left[\mathrm{g} / \mathrm{cm}^{2}\right]}\end{array}$ & $\begin{array}{c}\text { ANN Value } \\
{\left[\mathrm{g} / \mathrm{cm}^{2}\right]}\end{array}$ & $\begin{array}{c}\text { Sim. Difference } \\
{\left[\mathrm{g} / \mathrm{cm}^{2}\right]}\end{array}$ & $\begin{array}{c}\text { ANN Difference } \\
{\left[\mathrm{g} / \mathrm{cm}^{2}\right]}\end{array}$ \\
\hline \hline 0.10 & $13.48 \pm 2.97$ & $13.33 \pm 1.38$ & 3.42 & 3.57 \\
\hline 0.30 & $14.70 \pm 2.50$ & $15.27 \pm 1.41$ & 2.20 & 1.63 \\
\hline 0.50 & $15.77 \pm 2.28$ & $16.57 \pm 1.35$ & 1.13 & 0.33 \\
\hline 0.70 & $16.49 \pm 1.99$ & $17.59 \pm 1.36$ & 0.41 & -0.69 \\
\hline 0.90 & $17.16 \pm 1.91$ & $18.40 \pm 1.36$ & -0.26 & -1.50 \\
\hline
\end{tabular}

Table A.24 : Calculated simulation and ANN values using pmANN+BPM for iron in CNO-iron mixture of QGSJET showers at $\log (\mathrm{E} / \mathrm{eV})=20.00\left(\left\langle\mathrm{X}_{1}\right\rangle_{F e}=9.75 \pm 0.58 \mathrm{~g} / \mathrm{cm}^{2}\right)$

\begin{tabular}{|c|c|c|c|c|}
\hline $\mathrm{f}$ & $\begin{array}{c}\text { Sim. Value } \\
{\left[\mathrm{g} / \mathrm{cm}^{2}\right]}\end{array}$ & $\begin{array}{c}\text { ANN Value } \\
{\left[\mathrm{g} / \mathrm{cm}^{2}\right]}\end{array}$ & $\begin{array}{c}\text { Sim. Difference } \\
{\left[\mathrm{g} / \mathrm{cm}^{2}\right]}\end{array}$ & $\begin{array}{c}\text { ANN Difference } \\
{\left[\mathrm{g} / \mathrm{cm}^{2}\right]}\end{array}$ \\
\hline \hline 0.10 & $7.98 \pm 4.37$ & $6.57 \pm 5.48$ & 1.77 & 3.18 \\
\hline 0.30 & $9.20 \pm 4.94$ & $10.40 \pm 6.10$ & 0.55 & -0.65 \\
\hline 0.50 & $11.61 \pm 6.04$ & $12.13 \pm 7.77$ & -1.86 & -2.38 \\
\hline 0.70 & $13.84 \pm 6.71$ & $11.88 \pm 7.85$ & -4.09 & -2.13 \\
\hline 0.90 & $15.36 \pm 6.61$ & $13.29 \pm 9.34$ & -5.61 & -3.54 \\
\hline
\end{tabular}




\section{A.2 Results of pmANN+BPM for SIBYLL}

Table A.25 : Calculated simulation and ANN values using pmANN+BPM for $\mathrm{p}$ in $\mathrm{p}-\mathrm{CNO}$ mixture of SIBYLL showers at $\log (\mathrm{E} / \mathrm{eV})=18.75\left(\left\langle\mathrm{X}_{1}\right\rangle_{p}=37.78 \pm 1.38 \mathrm{~g} / \mathrm{cm}^{2}\right)$

\begin{tabular}{|c|c|c|c|c|}
\hline $\mathrm{f}$ & $\begin{array}{c}\text { Sim. Value } \\
{\left[\mathrm{g} / \mathrm{cm}^{2}\right]}\end{array}$ & $\begin{array}{c}\text { ANN Value } \\
{\left[\mathrm{g} / \mathrm{cm}^{2}\right]}\end{array}$ & $\begin{array}{c}\text { Sim. Difference } \\
{\left[\mathrm{g} / \mathrm{cm}^{2}\right]}\end{array}$ & $\begin{array}{c}\text { ANN Difference } \\
{\left[\mathrm{g} / \mathrm{cm}^{2}\right]}\end{array}$ \\
\hline \hline 0.10 & $20.97 \pm 1.61$ & $22.18 \pm 2.11$ & 16.81 & 15.60 \\
\hline 0.30 & $28.06 \pm 1.77$ & $28.22 \pm 2.27$ & 9.72 & 9.56 \\
\hline 0.50 & $38.06 \pm 5.57$ & $44.01 \pm 6.02$ & -0.28 & -6.23 \\
\hline 0.70 & $38.70 \pm 5.22$ & $45.19 \pm 5.82$ & -0.92 & -7.41 \\
\hline 0.90 & $39.44 \pm 4.95$ & $46.01 \pm 5.96$ & -1.66 & -8.23 \\
\hline
\end{tabular}

Table A.26 : Calculated simulation and ANN values using pmANN+BPM for CNO in p-CNO mixture of SIBYLL showers at $\log (\mathrm{E} / \mathrm{eV})=18.75\left(\left\langle\mathrm{X}_{1}\right\rangle_{C N O}=15.50 \pm 0.56 \mathrm{~g} / \mathrm{cm}^{2}\right)$

\begin{tabular}{|c|c|c|c|c|}
\hline $\mathrm{f}$ & $\begin{array}{c}\text { Sim. Value } \\
{\left[\mathrm{g} / \mathrm{cm}^{2}\right]}\end{array}$ & $\begin{array}{c}\text { ANN Value } \\
{\left[\mathrm{g} / \mathrm{cm}^{2}\right]}\end{array}$ & $\begin{array}{c}\text { Sim. Difference } \\
{\left[\mathrm{g} / \mathrm{cm}^{2}\right]}\end{array}$ & $\begin{array}{c}\text { ANN Difference } \\
{\left[\mathrm{g} / \mathrm{cm}^{2}\right]}\end{array}$ \\
\hline \hline 0.10 & $14.41 \pm 6.29$ & $16.64 \pm 5.02$ & 1.09 & -1.14 \\
\hline 0.30 & $18.41 \pm 7.75$ & $12.86 \pm 2.15$ & -2.91 & 2.64 \\
\hline 0.50 & $18.45 \pm 5.96$ & $18.13 \pm 1.97$ & -2.95 & -2.63 \\
\hline 0.70 & $21.00 \pm 7.17$ & $18.83 \pm 2.95$ & -5.50 & -3.33 \\
\hline 0.90 & $20.23 \pm 5.34$ & $20.22 \pm 5.32$ & -4.73 & -4.72 \\
\hline
\end{tabular}

Table A.27 : Calculated simulation and ANN values using pmANN+BPM for $\mathrm{p}$ in $\mathrm{p}-\mathrm{CNO}$ mixture of SIBYLL showers at $\log (\mathrm{E} / \mathrm{eV})=19.00\left(\left\langle\mathrm{X}_{1}\right\rangle_{p}=37.48 \pm 1.28 \mathrm{~g} / \mathrm{cm}^{2}\right)$

\begin{tabular}{|c|c|c|c|c|}
\hline $\mathrm{f}$ & $\begin{array}{c}\text { Sim. Value } \\
{\left[\mathrm{g} / \mathrm{cm}^{2}\right]}\end{array}$ & $\begin{array}{c}\text { ANN Value } \\
{\left[\mathrm{g} / \mathrm{cm}^{2}\right]}\end{array}$ & $\begin{array}{c}\text { Sim. Difference } \\
{\left[\mathrm{g} / \mathrm{cm}^{2}\right]}\end{array}$ & $\begin{array}{c}\text { ANN Difference } \\
{\left[\mathrm{g} / \mathrm{cm}^{2}\right]}\end{array}$ \\
\hline \hline 0.10 & $20.57 \pm 1.97$ & $20.42 \pm 2.13$ & 16.91 & 17.06 \\
\hline 0.30 & $27.05 \pm 2.04$ & $26.02 \pm 2.44$ & 10.43 & 11.46 \\
\hline 0.50 & $35.89 \pm 5.77$ & $44.60 \pm 7.98$ & 1.59 & -7.12 \\
\hline 0.70 & $36.77 \pm 5.52$ & $45.32 \pm 7.12$ & 0.71 & -7.84 \\
\hline 0.90 & $37.47 \pm 5.06$ & $44.93 \pm 6.94$ & 0.01 & -7.45 \\
\hline
\end{tabular}


Table A.28 : Calculated simulation and ANN values using pmANN+BPM for CNO in p-CNO mixture of SIBYLL showers at $\log (\mathrm{E} / \mathrm{eV})=19.00\left(\left\langle\mathrm{X}_{1}\right\rangle_{C N O}=15.48 \pm 0.55 \mathrm{~g} / \mathrm{cm}^{2}\right)$

\begin{tabular}{|c|c|c|c|c|}
\hline $\mathrm{f}$ & $\begin{array}{c}\text { Sim. Value } \\
{\left[\mathrm{g} / \mathrm{cm}^{2}\right]}\end{array}$ & $\begin{array}{c}\text { ANN Value } \\
{\left[\mathrm{g} / \mathrm{cm}^{2}\right]}\end{array}$ & $\begin{array}{c}\text { Sim. Difference } \\
{\left[\mathrm{g} / \mathrm{cm}^{2}\right]}\end{array}$ & $\begin{array}{c}\text { ANN Difference } \\
{\left[\mathrm{g} / \mathrm{cm}^{2}\right]}\end{array}$ \\
\hline \hline 0.10 & $15.07 \pm 7.28$ & $17.51 \pm 5.30$ & 0.41 & -2.03 \\
\hline 0.30 & $17.50 \pm 8.00$ & $14.43 \pm 3.07$ & -2.02 & 1.05 \\
\hline 0.50 & $18.11 \pm 5.79$ & $18.28 \pm 1.97$ & -2.63 & -2.80 \\
\hline 0.70 & $21.52 \pm 7.01$ & $19.49 \pm 2.76$ & -6.04 & -4.01 \\
\hline 0.90 & $21.42 \pm 5.61$ & $21.60 \pm 4.87$ & -5.94 & -6.12 \\
\hline
\end{tabular}

Table A.29 : Calculated simulation and ANN values using pmANN+BPM for $\mathrm{p}$ in $\mathrm{p}-\mathrm{CNO}$ mixture of SIBYLL showers at $\log (\mathrm{E} / \mathrm{eV})=19.50\left(\left\langle\mathrm{X}_{1}\right\rangle_{p}=34.04 \pm 1.10 \mathrm{~g} / \mathrm{cm}^{2}\right)$

\begin{tabular}{|c|c|c|c|c|}
\hline $\mathrm{f}$ & $\begin{array}{c}\text { Sim. Value } \\
{\left[\mathrm{g} / \mathrm{cm}^{2}\right]}\end{array}$ & $\begin{array}{c}\text { ANN Value } \\
{\left[\mathrm{g} / \mathrm{cm}^{2}\right]}\end{array}$ & $\begin{array}{c}\text { Sim. Difference } \\
{\left[\mathrm{g} / \mathrm{cm}^{2}\right]}\end{array}$ & $\begin{array}{c}\text { ANN Difference } \\
{\left[\mathrm{g} / \mathrm{cm}^{2}\right]}\end{array}$ \\
\hline \hline 0.10 & $18.84 \pm 2.41$ & $20.66 \pm 2.40$ & 15.20 & 13.38 \\
\hline 0.30 & $25.29 \pm 3.02$ & $25.25 \pm 2.61$ & 8.75 & 8.79 \\
\hline 0.50 & $36.42 \pm 7.38$ & $34.89 \pm 7.12$ & -2.38 & -0.85 \\
\hline 0.70 & $34.06 \pm 5.57$ & $36.80 \pm 6.26$ & -0.02 & -2.76 \\
\hline 0.90 & $33.30 \pm 4.87$ & $37.77 \pm 6.21$ & 0.74 & -3.73 \\
\hline
\end{tabular}

Table A.30 : Calculated simulation and ANN values using pmANN+BPM for CNO in p-CNO mixture of SIBYLL showers at $\log (\mathrm{E} / \mathrm{eV})=19.50\left(\left\langle\mathrm{X}_{1}\right\rangle_{C N O}=14.13 \pm 0.64 \mathrm{~g} / \mathrm{cm}^{2}\right)$

\begin{tabular}{|c|c|c|c|c|}
\hline $\mathrm{f}$ & $\begin{array}{c}\text { Sim. Value } \\
{\left[\mathrm{g} / \mathrm{cm}^{2}\right]}\end{array}$ & $\begin{array}{c}\text { ANN Value } \\
{\left[\mathrm{g} / \mathrm{cm}^{2}\right]}\end{array}$ & $\begin{array}{c}\text { Sim. Difference } \\
{\left[\mathrm{g} / \mathrm{cm}^{2}\right]}\end{array}$ & $\begin{array}{c}\text { ANN Difference } \\
{\left[\mathrm{g} / \mathrm{cm}^{2}\right]}\end{array}$ \\
\hline \hline 0.10 & $15.19 \pm 7.55$ & $14.49 \pm 2.80$ & -1.06 & -0.36 \\
\hline 0.30 & $13.99 \pm 6.85$ & $14.44 \pm 2.40$ & 0.14 & -0.31 \\
\hline 0.50 & $17.34 \pm 4.17$ & $16.49 \pm 2.99$ & -3.21 & -2.36 \\
\hline 0.70 & $20.23 \pm 5.66$ & $18.16 \pm 4.00$ & -6.10 & -4.03 \\
\hline 0.90 & $22.25 \pm 6.03$ & $19.62 \pm 4.60$ & -8.12 & -5.49 \\
\hline
\end{tabular}

Table A.31 : Calculated simulation and ANN values using pmANN+BPM for $\mathrm{p}$ in $\mathrm{p}-\mathrm{CNO}$ mixture of SIBYLL showers at $\log (\mathrm{E} / \mathrm{eV})=20.00\left(\left\langle\mathrm{X}_{1}\right\rangle_{p}=32.29 \pm 1.01 \mathrm{~g} / \mathrm{cm}^{2}\right)$

\begin{tabular}{|c|c|c|c|c|}
\hline $\mathrm{f}$ & $\begin{array}{c}\text { Sim. Value } \\
{\left[\mathrm{g} / \mathrm{cm}^{2}\right]}\end{array}$ & $\begin{array}{c}\text { ANN Value } \\
{\left[\mathrm{g} / \mathrm{cm}^{2}\right]}\end{array}$ & $\begin{array}{c}\text { Sim. Difference } \\
{\left[\mathrm{g} / \mathrm{cm}^{2}\right]}\end{array}$ & $\begin{array}{c}\text { ANN Difference } \\
{\left[\mathrm{g} / \mathrm{cm}^{2}\right]}\end{array}$ \\
\hline \hline 0.10 & $17.53 \pm 1.83$ & $18.12 \pm 2.21$ & 14.76 & 14.17 \\
\hline 0.30 & $22.27 \pm 1.96$ & $21.96 \pm 2.46$ & 10.02 & 10.33 \\
\hline 0.50 & $29.93 \pm 7.18$ & $33.01 \pm 11.34$ & 2.36 & -0.72 \\
\hline 0.70 & $31.31 \pm 5.83$ & $40.94 \pm 10.82$ & 0.98 & -8.65 \\
\hline 0.90 & $30.69 \pm 4.97$ & $42.45 \pm 10.28$ & 1.60 & -10.16 \\
\hline
\end{tabular}


Table A.32 : Calculated simulation and ANN values using pmANN+BPM for CNO in p-CNO mixture of SIBYLL showers at $\log (\mathrm{E} / \mathrm{eV})=20.00\left(\left\langle\mathrm{X}_{1}\right\rangle_{C N O}=15.09 \pm 0.46 \mathrm{~g} / \mathrm{cm}^{2}\right)$

\begin{tabular}{|c|c|c|c|c|}
\hline $\mathrm{f}$ & $\begin{array}{c}\text { Sim. Value } \\
{\left[\mathrm{g} / \mathrm{cm}^{2}\right]}\end{array}$ & $\begin{array}{c}\text { ANN Value } \\
{\left[\mathrm{g} / \mathrm{cm}^{2}\right]}\end{array}$ & $\begin{array}{c}\text { Sim. Difference } \\
{\left[\mathrm{g} / \mathrm{cm}^{2}\right]}\end{array}$ & $\begin{array}{c}\text { ANN Difference } \\
{\left[\mathrm{g} / \mathrm{cm}^{2}\right]}\end{array}$ \\
\hline \hline 0.10 & $18.39 \pm 7.03$ & $15.54 \pm 5.11$ & -3.30 & -0.45 \\
\hline 0.30 & $17.46 \pm 8.02$ & $15.19 \pm 3.64$ & -2.37 & -0.10 \\
\hline 0.50 & $18.23 \pm 5.90$ & $17.94 \pm 3.46$ & -3.14 & -2.85 \\
\hline 0.70 & $18.58 \pm 4.02$ & $19.60 \pm 2.95$ & -3.49 & -4.51 \\
\hline 0.90 & $19.83 \pm 5.32$ & $21.71 \pm 3.02$ & -4.74 & -6.62 \\
\hline
\end{tabular}

Table A.33 : Calculated simulation and ANN values using pmANN+BPM for $\mathrm{p}$ in $\mathrm{p}$-iron mixture of SIBYLL showers at $\log (\mathrm{E} / \mathrm{eV})=18.75\left(\left\langle\mathrm{X}_{1}\right\rangle_{p}=37.78 \pm 1.38 \mathrm{~g} / \mathrm{cm}^{2}\right)$

\begin{tabular}{|c|c|c|c|c|}
\hline $\mathrm{f}$ & $\begin{array}{c}\text { Sim. Value } \\
{\left[\mathrm{g} / \mathrm{cm}^{2}\right]}\end{array}$ & $\begin{array}{c}\text { ANN Value } \\
{\left[\mathrm{g} / \mathrm{cm}^{2}\right]}\end{array}$ & $\begin{array}{c}\text { Sim. Difference } \\
{\left[\mathrm{g} / \mathrm{cm}^{2}\right]}\end{array}$ & $\begin{array}{c}\text { ANN Difference } \\
{\left[\mathrm{g} / \mathrm{cm}^{2}\right]}\end{array}$ \\
\hline \hline 0.10 & $18.47 \pm 2.86$ & $20.12 \pm 2.51$ & 19.31 & 17.66 \\
\hline 0.30 & $29.42 \pm 2.33$ & $30.51 \pm 2.84$ & 8.36 & 7.27 \\
\hline 0.50 & $39.04 \pm 6.04$ & $47.62 \pm 7.50$ & -1.26 & -9.84 \\
\hline 0.70 & $39.18 \pm 5.29$ & $47.19 \pm 6.43$ & -1.40 & -9.41 \\
\hline 0.90 & $39.53 \pm 4.98$ & $46.29 \pm 5.96$ & -1.75 & -8.51 \\
\hline
\end{tabular}

Table A.34 : Calculated simulation and ANN values using pmANN+BPM for iron in p-iron mixture of SIBYLL showers at $\log (\mathrm{E} / \mathrm{eV})=18.75\left(\left\langle\mathrm{X}_{1}\right\rangle_{\mathrm{Fe}}=9.18 \pm 0.58 \mathrm{~g} / \mathrm{cm}^{2}\right)$

\begin{tabular}{|c|c|c|c|c|}
\hline $\mathrm{f}$ & $\begin{array}{c}\text { Sim. Value } \\
{\left[\mathrm{g} / \mathrm{cm}^{2}\right]}\end{array}$ & $\begin{array}{c}\text { ANN Value } \\
{\left[\mathrm{g} / \mathrm{cm}^{2}\right]}\end{array}$ & $\begin{array}{c}\text { Sim. Difference } \\
{\left[\mathrm{g} / \mathrm{cm}^{2}\right]}\end{array}$ & $\begin{array}{c}\text { ANN Difference } \\
{\left[\mathrm{g} / \mathrm{cm}^{2}\right]}\end{array}$ \\
\hline \hline 0.10 & $6.52 \pm 1.30$ & $6.32 \pm 1.00$ & 2.66 & 2.86 \\
\hline 0.30 & $8.29 \pm 2.75$ & $7.18 \pm 1.08$ & 0.89 & 2.00 \\
\hline 0.50 & $14.18 \pm 6.20$ & $10.68 \pm 1.68$ & -5.00 & -1.50 \\
\hline 0.70 & $17.52 \pm 7.80$ & $11.41 \pm 2.56$ & -8.34 & -2.23 \\
\hline 0.90 & $20.20 \pm 5.59$ & $13.91 \pm 4.93$ & -11.02 & -4.73 \\
\hline
\end{tabular}

Table A.35 : Calculated simulation and ANN values using pmANN+BPM for $\mathrm{p}$ in $\mathrm{p}$-iron mixture of SIBYLL showers at $\log (\mathrm{E} / \mathrm{eV})=19.00\left(\left\langle\mathrm{X}_{1}\right\rangle_{p}=37.48 \pm 1.28 \mathrm{~g} / \mathrm{cm}^{2}\right)$

\begin{tabular}{|c|c|c|c|c|}
\hline $\mathrm{f}$ & $\begin{array}{c}\text { Sim. Value } \\
{\left[\mathrm{g} / \mathrm{cm}^{2}\right]}\end{array}$ & $\begin{array}{c}\text { ANN Value } \\
{\left[\mathrm{g} / \mathrm{cm}^{2}\right]}\end{array}$ & $\begin{array}{c}\text { Sim. Difference } \\
{\left[\mathrm{g} / \mathrm{cm}^{2}\right]}\end{array}$ & $\begin{array}{c}\text { ANN Difference } \\
{\left[\mathrm{g} / \mathrm{cm}^{2}\right]}\end{array}$ \\
\hline \hline 0.10 & $19.62 \pm 3.52$ & $19.56 \pm 2.77$ & 17.86 & 17.92 \\
\hline 0.30 & $29.09 \pm 2.68$ & $28.73 \pm 3.12$ & 8.39 & 8.75 \\
\hline 0.50 & $37.91 \pm 7.01$ & $46.03 \pm 10.16$ & -0.43 & -8.55 \\
\hline 0.70 & $38.32 \pm 5.69$ & $46.98 \pm 7.40$ & -0.84 & -9.50 \\
\hline 0.90 & $38.59 \pm 5.26$ & $45.36 \pm 6.82$ & -1.11 & -7.88 \\
\hline
\end{tabular}


Table A.36 : Calculated simulation and ANN values using pmANN+BPM for iron in p-iron mixture of SIBYLL showers at $\log (\mathrm{E} / \mathrm{eV})=19.00\left(\left\langle\mathrm{X}_{1}\right\rangle_{F e}=9.42 \pm 0.56 \mathrm{~g} / \mathrm{cm}^{2}\right)$

\begin{tabular}{|c|c|c|c|c|}
\hline $\mathrm{f}$ & $\begin{array}{c}\text { Sim. Value } \\
{\left[\mathrm{g} / \mathrm{cm}^{2}\right]}\end{array}$ & $\begin{array}{c}\text { ANN Value } \\
{\left[\mathrm{g} / \mathrm{cm}^{2}\right]}\end{array}$ & $\begin{array}{c}\text { Sim. Difference } \\
{\left[\mathrm{g} / \mathrm{cm}^{2}\right]}\end{array}$ & $\begin{array}{c}\text { ANN Difference } \\
{\left[\mathrm{g} / \mathrm{cm}^{2}\right]}\end{array}$ \\
\hline \hline 0.10 & $7.36 \pm 1.65$ & $6.09 \pm 1.01$ & 2.06 & 3.33 \\
\hline 0.30 & $9.89 \pm 4.59$ & $6.79 \pm 1.11$ & -0.47 & 2.63 \\
\hline 0.50 & $15.89 \pm 6.71$ & $10.43 \pm 1.99$ & -6.47 & -1.01 \\
\hline 0.70 & $20.63 \pm 7.00$ & $12.14 \pm 2.48$ & -11.21 & -2.72 \\
\hline 0.90 & $21.84 \pm 5.34$ & $15.77 \pm 4.97$ & -12.42 & -6.35 \\
\hline
\end{tabular}

Table A.37 : Calculated simulation and ANN values using pmANN+BPM for $\mathrm{p}$ in $\mathrm{p}$-iron mixture of SIBYLL showers at $\log (\mathrm{E} / \mathrm{eV})=19.50\left(\left\langle\mathrm{X}_{1}\right\rangle_{p}=34.04 \pm 1.10 \mathrm{~g} / \mathrm{cm}^{2}\right)$

\begin{tabular}{|c|c|c|c|c|}
\hline $\mathrm{f}$ & $\begin{array}{c}\text { Sim. Value } \\
{\left[\mathrm{g} / \mathrm{cm}^{2}\right]}\end{array}$ & $\begin{array}{c}\text { ANN Value } \\
{\left[\mathrm{g} / \mathrm{cm}^{2}\right]}\end{array}$ & $\begin{array}{c}\text { Sim. Difference } \\
{\left[\mathrm{g} / \mathrm{cm}^{2}\right]}\end{array}$ & $\begin{array}{c}\text { ANN Difference } \\
{\left[\mathrm{g} / \mathrm{cm}^{2}\right]}\end{array}$ \\
\hline \hline 0.10 & $19.37 \pm 5.33$ & $18.88 \pm 3.60$ & 14.67 & 15.16 \\
\hline 0.30 & $27.39 \pm 3.51$ & $26.75 \pm 3.39$ & 6.65 & 7.29 \\
\hline 0.50 & $36.13 \pm 7.97$ & $37.59 \pm 9.45$ & -2.09 & -3.55 \\
\hline 0.70 & $35.42 \pm 5.56$ & $39.80 \pm 7.63$ & -1.38 & -5.76 \\
\hline 0.90 & $34.00 \pm 4.75$ & $38.41 \pm 6.22$ & 0.04 & -4.37 \\
\hline
\end{tabular}

Table A.38 : Calculated simulation and ANN values using pmANN+BPM for iron in p-iron mixture of SIBYLL showers at $\log (\mathrm{E} / \mathrm{eV})=19.50\left(\left\langle\mathrm{X}_{1}\right\rangle_{F e}=9.35 \pm 0.68 \mathrm{~g} / \mathrm{cm}^{2}\right)$

\begin{tabular}{|c|c|c|c|c|}
\hline $\mathrm{f}$ & $\begin{array}{c}\text { Sim. Value } \\
{\left[\mathrm{g} / \mathrm{cm}^{2}\right]}\end{array}$ & $\begin{array}{c}\text { ANN Value } \\
{\left[\mathrm{g} / \mathrm{cm}^{2}\right]}\end{array}$ & $\begin{array}{c}\text { Sim. Difference } \\
{\left[\mathrm{g} / \mathrm{cm}^{2}\right]}\end{array}$ & $\begin{array}{c}\text { ANN Difference } \\
{\left[\mathrm{g} / \mathrm{cm}^{2}\right]}\end{array}$ \\
\hline \hline 0.10 & $7.38 \pm 2.98$ & $6.14 \pm 1.09$ & 1.97 & 3.21 \\
\hline 0.30 & $9.60 \pm 4.26$ & $6.74 \pm 1.14$ & -0.25 & 2.61 \\
\hline 0.50 & $16.14 \pm 5.40$ & $8.99 \pm 2.26$ & -6.79 & 0.36 \\
\hline 0.70 & $20.15 \pm 6.21$ & $11.39 \pm 3.56$ & -10.80 & -2.04 \\
\hline 0.90 & $22.33 \pm 6.48$ & $14.77 \pm 4.72$ & -12.98 & -5.42 \\
\hline
\end{tabular}

Table A.39 : Calculated simulation and ANN values using pmANN+BPM for $\mathrm{p}$ in $\mathrm{p}$-iron mixture of SIBYLL showers at $\log (\mathrm{E} / \mathrm{eV})=20.00\left(\left\langle\mathrm{X}_{1}\right\rangle_{p}=32.29 \pm 1.01 \mathrm{~g} / \mathrm{cm}^{2}\right)$

\begin{tabular}{|c|c|c|c|c|}
\hline $\mathrm{f}$ & $\begin{array}{c}\text { Sim. Value } \\
{\left[\mathrm{g} / \mathrm{cm}^{2}\right]}\end{array}$ & $\begin{array}{c}\text { ANN Value } \\
{\left[\mathrm{g} / \mathrm{cm}^{2}\right]}\end{array}$ & $\begin{array}{c}\text { Sim. Difference } \\
{\left[\mathrm{g} / \mathrm{cm}^{2}\right]}\end{array}$ & $\begin{array}{c}\text { ANN Difference } \\
{\left[\mathrm{g} / \mathrm{cm}^{2}\right]}\end{array}$ \\
\hline \hline 0.10 & $22.74 \pm 11.19$ & $13.91 \pm 3.25$ & 9.55 & 18.38 \\
\hline 0.30 & $28.56 \pm 3.99$ & $23.00 \pm 3.43$ & 3.73 & 9.29 \\
\hline 0.50 & $32.04 \pm 6.76$ & $30.65 \pm 9.60$ & 0.25 & 1.64 \\
\hline 0.70 & $34.90 \pm 6.67$ & $42.88 \pm 10.30$ & -2.61 & -10.59 \\
\hline 0.90 & $32.52 \pm 5.02$ & $41.88 \pm 9.20$ & -0.23 & -9.59 \\
\hline
\end{tabular}


Table A.40 : Calculated simulation and ANN values using pmANN+BPM for iron in p-iron mixture of SIBYLL showers at $\log (\mathrm{E} / \mathrm{eV})=20.00\left(\left\langle\mathrm{X}_{1}\right\rangle_{F e}=9.16 \pm 1.03 \mathrm{~g} / \mathrm{cm}^{2}\right)$

\begin{tabular}{|c|c|c|c|c|}
\hline $\mathrm{f}$ & $\begin{array}{c}\text { Sim. Value } \\
{\left[\mathrm{g} / \mathrm{cm}^{2}\right]}\end{array}$ & $\begin{array}{c}\text { ANN Value } \\
{\left[\mathrm{g} / \mathrm{cm}^{2}\right]}\end{array}$ & $\begin{array}{c}\text { Sim. Difference } \\
{\left[\mathrm{g} / \mathrm{cm}^{2}\right]}\end{array}$ & $\begin{array}{c}\text { ANN Difference } \\
{\left[\mathrm{g} / \mathrm{cm}^{2}\right]}\end{array}$ \\
\hline \hline 0.10 & $8.12 \pm 2.52$ & $8.59 \pm 1.81$ & 1.04 & 0.57 \\
\hline 0.30 & $10.99 \pm 5.29$ & $8.93 \pm 1.17$ & -1.83 & 0.23 \\
\hline 0.50 & $15.90 \pm 6.82$ & $10.65 \pm 2.10$ & -6.74 & -1.49 \\
\hline 0.70 & $20.18 \pm 5.36$ & $14.32 \pm 2.52$ & -11.02 & -5.16 \\
\hline 0.90 & $21.71 \pm 5.66$ & $17.38 \pm 3.13$ & -12.55 & -8.22 \\
\hline
\end{tabular}

Table A.41 : Calculated simulation and ANN values using pmANN+BPM for CNO in CNO-iron mixture of SIBYLL showers at $\log (\mathrm{E} / \mathrm{eV})=18.75\left(\left\langle\mathrm{X}_{1}\right\rangle_{C N O}=15.50 \pm 0.56 \mathrm{~g} / \mathrm{cm}^{2}\right)$

\begin{tabular}{|c|c|c|c|c|}
\hline $\mathrm{f}$ & $\begin{array}{c}\text { Sim. Value } \\
{\left[\mathrm{g} / \mathrm{cm}^{2}\right]}\end{array}$ & $\begin{array}{c}\text { ANN Value } \\
{\left[\mathrm{g} / \mathrm{cm}^{2}\right]}\end{array}$ & $\begin{array}{c}\text { Sim. Difference } \\
{\left[\mathrm{g} / \mathrm{cm}^{2}\right]}\end{array}$ & $\begin{array}{c}\text { ANN Difference } \\
{\left[\mathrm{g} / \mathrm{cm}^{2}\right]}\end{array}$ \\
\hline \hline 0.10 & $10.37 \pm 1.59$ & $15.83 \pm 1.27$ & 5.13 & -0.33 \\
\hline 0.30 & $12.79 \pm 1.49$ & $17.20 \pm 1.22$ & 2.71 & -1.70 \\
\hline 0.50 & $14.45 \pm 1.45$ & $18.06 \pm 1.18$ & 1.05 & -2.56 \\
\hline 0.70 & $15.58 \pm 1.41$ & $18.59 \pm 1.17$ & -0.08 & -3.09 \\
\hline 0.90 & $16.41 \pm 1.34$ & $18.98 \pm 1.13$ & -0.91 & -3.48 \\
\hline
\end{tabular}

Table A.42 : Calculated simulation and ANN values using pmANN+BPM for iron in CNO-iron mixture of SIBYLL showers at $\log (\mathrm{E} / \mathrm{eV})=18.75\left(\left\langle\mathrm{X}_{1}\right\rangle_{\mathrm{Fe}}=9.18 \pm 0.56 \mathrm{~g} / \mathrm{cm}^{2}\right)$

\begin{tabular}{|c|c|c|c|c|}
\hline $\mathrm{f}$ & $\begin{array}{c}\text { Sim. Value } \\
{\left[\mathrm{g} / \mathrm{cm}^{2}\right]}\end{array}$ & $\begin{array}{c}\text { ANN Value } \\
{\left[\mathrm{g} / \mathrm{cm}^{2}\right]}\end{array}$ & $\begin{array}{c}\text { Sim. Difference } \\
{\left[\mathrm{g} / \mathrm{cm}^{2}\right]}\end{array}$ & $\begin{array}{c}\text { ANN Difference } \\
{\left[\mathrm{g} / \mathrm{cm}^{2}\right]}\end{array}$ \\
\hline \hline 0.10 & $9.08 \pm 5.59$ & $8.62 \pm 5.73$ & 0.10 & 0.56 \\
\hline 0.30 & $9.76 \pm 6.22$ & $8.47 \pm 5.63$ & -0.58 & 0.71 \\
\hline 0.50 & $10.74 \pm 6.51$ & $11.10 \pm 7.62$ & -1.56 & -1.92 \\
\hline 0.70 & $11.90 \pm 6.67$ & $11.85 \pm 6.61$ & -2.72 & -2.67 \\
\hline 0.90 & $13.95 \pm 7.21$ & $16.88 \pm 6.37$ & -4.77 & -7.70 \\
\hline
\end{tabular}


Table A.43 : Calculated simulation and ANN values using pmANN+BPM for CNO in CNO-iron mixture of SIBYLL showers at $\log (\mathrm{E} / \mathrm{eV})=19.00\left(\left\langle\mathrm{X}_{1}\right\rangle_{C N O}=15.48 \pm 0.55 \mathrm{~g} / \mathrm{cm}^{2}\right)$

\begin{tabular}{|c|c|c|c|c|}
\hline $\mathrm{f}$ & $\begin{array}{c}\text { Sim. Value } \\
{\left[\mathrm{g} / \mathrm{cm}^{2}\right]}\end{array}$ & $\begin{array}{c}\text { ANN Value } \\
{\left[\mathrm{g} / \mathrm{cm}^{2}\right]}\end{array}$ & $\begin{array}{c}\text { Sim. Difference } \\
{\left[\mathrm{g} / \mathrm{cm}^{2}\right]}\end{array}$ & $\begin{array}{c}\text { ANN Difference } \\
{\left[\mathrm{g} / \mathrm{cm}^{2}\right]}\end{array}$ \\
\hline \hline 0.10 & $11.31 \pm 2.00$ & $15.37 \pm 1.53$ & 4.17 & 0.11 \\
\hline 0.30 & $12.91 \pm 1.99$ & $16.56 \pm 1.50$ & 2.57 & -1.08 \\
\hline 0.50 & $14.16 \pm 1.90$ & $17.39 \pm 1.38$ & 1.32 & -1.91 \\
\hline 0.70 & $15.08 \pm 1.87$ & $17.88 \pm 1.28$ & 0.40 & -2.40 \\
\hline 0.90 & $15.79 \pm 1.76$ & $18.22 \pm 1.21$ & -0.31 & -2.74 \\
\hline
\end{tabular}

Table A.44 : Calculated simulation and ANN values using pmANN+BPM for iron in CNO-iron mixture of SIBYLL showers at $\log (\mathrm{E} / \mathrm{eV})=19.00\left(\left\langle\mathrm{X}_{1}\right\rangle_{\mathrm{Fe}}=9.42 \pm 0.56 \mathrm{~g} / \mathrm{cm}^{2}\right)$

\begin{tabular}{|c|c|c|c|c|}
\hline $\mathrm{f}$ & $\begin{array}{c}\text { Sim. Value } \\
{\left[\mathrm{g} / \mathrm{cm}^{2}\right]}\end{array}$ & $\begin{array}{c}\text { ANN Value } \\
{\left[\mathrm{g} / \mathrm{cm}^{2}\right]}\end{array}$ & $\begin{array}{c}\text { Sim. Difference } \\
{\left[\mathrm{g} / \mathrm{cm}^{2}\right]}\end{array}$ & $\begin{array}{c}\text { ANN Difference } \\
{\left[\mathrm{g} / \mathrm{cm}^{2}\right]}\end{array}$ \\
\hline \hline 0.10 & $8.47 \pm 4.87$ & $6.69 \pm 4.21$ & 0.95 & 2.73 \\
\hline 0.30 & $9.52 \pm 5.46$ & $7.32 \pm 4.85$ & -0.10 & 2.10 \\
\hline 0.50 & $11.13 \pm 5.94$ & $8.17 \pm 5.17$ & -1.71 & 1.25 \\
\hline 0.70 & $13.29 \pm 6.83$ & $12.38 \pm 7.23$ & -3.87 & -2.96 \\
\hline 0.90 & $15.22 \pm 7.23$ & $14.26 \pm 4.69$ & -5.80 & -4.84 \\
\hline
\end{tabular}

Table A.45 : Calculated simulation and ANN values using pmANN+BPM for CNO in CNO-iron mixture of SIBYLL showers at $\log (\mathrm{E} / \mathrm{eV})=19.50\left(\left\langle\mathrm{X}_{1}\right\rangle_{\mathrm{CNO}}=14.13 \pm 0.64 \mathrm{~g} / \mathrm{cm}^{2}\right)$

\begin{tabular}{|c|c|c|c|c|}
\hline $\mathrm{f}$ & $\begin{array}{c}\text { Sim. Value } \\
{\left[\mathrm{g} / \mathrm{cm}^{2}\right]}\end{array}$ & $\begin{array}{c}\text { ANN Value } \\
{\left[\mathrm{g} / \mathrm{cm}^{2}\right]}\end{array}$ & $\begin{array}{c}\text { Sim. Difference } \\
{\left[\mathrm{g} / \mathrm{cm}^{2}\right]}\end{array}$ & $\begin{array}{c}\text { ANN Difference } \\
{\left[\mathrm{g} / \mathrm{cm}^{2}\right]}\end{array}$ \\
\hline \hline 0.10 & $11.86 \pm 3.30$ & $14.63 \pm 2.20$ & 2.27 & -0.50 \\
\hline 0.30 & $12.89 \pm 2.62$ & $16.11 \pm 1.99$ & 1.24 & -1.98 \\
\hline 0.50 & $13.58 \pm 2.30$ & $17.11 \pm 1.90$ & 0.55 & -2.98 \\
\hline 0.70 & $14.43 \pm 2.27$ & $17.93 \pm 1.81$ & -0.30 & -3.80 \\
\hline 0.90 & $14.93 \pm 2.08$ & $18.50 \pm 1.79$ & -0.80 & -4.37 \\
\hline
\end{tabular}


Table A.46 : Calculated simulation and ANN values using pmANN+BPM for iron in CNO-iron mixture of SIBYLL showers at $\log (\mathrm{E} / \mathrm{eV})=19.50\left(\left\langle\mathrm{X}_{1}\right\rangle_{\mathrm{Fe}}=9.35 \pm 0.68 \mathrm{~g} / \mathrm{cm}^{2}\right)$

\begin{tabular}{|c|c|c|c|c|}
\hline $\mathrm{f}$ & $\begin{array}{c}\text { Sim. Value } \\
{\left[\mathrm{g} / \mathrm{cm}^{2}\right]}\end{array}$ & $\begin{array}{c}\text { ANN Value } \\
{\left[\mathrm{g} / \mathrm{cm}^{2}\right]}\end{array}$ & $\begin{array}{c}\text { Sim. Difference } \\
{\left[\mathrm{g} / \mathrm{cm}^{2}\right]}\end{array}$ & $\begin{array}{c}\text { ANN Difference } \\
{\left[\mathrm{g} / \mathrm{cm}^{2}\right]}\end{array}$ \\
\hline \hline 0.10 & $7.58 \pm 4.64$ & $6.12 \pm 2.54$ & 1.77 & 3.23 \\
\hline 0.30 & $9.19 \pm 5.34$ & $6.66 \pm 2.20$ & 0.16 & 2.69 \\
\hline 0.50 & $10.79 \pm 6.37$ & $7.92 \pm 2.86$ & -1.44 & 1.43 \\
\hline 0.70 & $11.89 \pm 6.43$ & $9.61 \pm 3.53$ & -2.54 & -0.26 \\
\hline 0.90 & $13.03 \pm 6.67$ & $11.86 \pm 3.76$ & -3.68 & -2.51 \\
\hline
\end{tabular}

Table A.47 : Calculated simulation and ANN values using pmANN+BPM for CNO in CNO-iron mixture of SIBYLL showers at $\log (\mathrm{E} / \mathrm{eV})=20.00\left(\left\langle\mathrm{X}_{1}\right\rangle_{C N O}=15.09 \pm 0.46 \mathrm{~g} / \mathrm{cm}^{2}\right)$

\begin{tabular}{|c|c|c|c|c|}
\hline $\mathrm{f}$ & $\begin{array}{c}\text { Sim. Value } \\
{\left[\mathrm{g} / \mathrm{cm}^{2}\right]}\end{array}$ & $\begin{array}{c}\text { ANN Value } \\
{\left[\mathrm{g} / \mathrm{cm}^{2}\right]}\end{array}$ & $\begin{array}{c}\text { Sim. Difference } \\
{\left[\mathrm{g} / \mathrm{cm}^{2}\right]}\end{array}$ & $\begin{array}{c}\text { ANN Difference } \\
{\left[\mathrm{g} / \mathrm{cm}^{2}\right]}\end{array}$ \\
\hline \hline 0.10 & $9.22 \pm 3.59$ & $12.82 \pm 1.69$ & 5.87 & 2.27 \\
\hline 0.30 & $11.86 \pm 2.83$ & $14.64 \pm 1.71$ & 3.23 & 0.45 \\
\hline 0.50 & $13.26 \pm 2.29$ & $16.05 \pm 1.64$ & 1.83 & -0.96 \\
\hline 0.70 & $14.01 \pm 1.90$ & $17.02 \pm 1.56$ & 1.08 & -1.93 \\
\hline 0.90 & $14.53 \pm 1.68$ & $17.76 \pm 1.50$ & 0.56 & -2.67 \\
\hline
\end{tabular}

Table A.48 : Calculated simulation and ANN values using pmANN+BPM for iron in CNO-iron mixture of SIBYLL showers at $\log (\mathrm{E} / \mathrm{eV})=20.00\left(\left\langle\mathrm{X}_{1}\right\rangle_{\mathrm{Fe}}=9.16 \pm 1.03 \mathrm{~g} / \mathrm{cm}^{2}\right)$

\begin{tabular}{|c|c|c|c|c|}
\hline $\mathrm{f}$ & $\begin{array}{c}\text { Sim. Value } \\
{\left[\mathrm{g} / \mathrm{cm}^{2}\right]}\end{array}$ & $\begin{array}{c}\text { ANN Value } \\
{\left[\mathrm{g} / \mathrm{cm}^{2}\right]}\end{array}$ & $\begin{array}{c}\text { Sim. Difference } \\
{\left[\mathrm{g} / \mathrm{cm}^{2}\right]}\end{array}$ & $\begin{array}{c}\text { ANN Difference } \\
{\left[\mathrm{g} / \mathrm{cm}^{2}\right]}\end{array}$ \\
\hline \hline 0.10 & $5.60 \pm 3.18$ & $7.69 \pm 4.22$ & 3.56 & 1.47 \\
\hline 0.30 & $9.28 \pm 5.08$ & $9.36 \pm 3.96$ & -0.12 & -0.20 \\
\hline 0.50 & $11.97 \pm 6.37$ & $10.55 \pm 4.35$ & -2.81 & -1.39 \\
\hline 0.70 & $13.33 \pm 6.54$ & $10.91 \pm 4.25$ & -4.17 & -1.75 \\
\hline 0.90 & $14.63 \pm 6.82$ & $12.59 \pm 5.77$ & -5.47 & -3.43 \\
\hline
\end{tabular}




\section{A.3 Results of pmANN+BPM for EPOS}

Table A.49 : Calculated simulation and ANN values using pmANN+BPM for CNO in p-CNO mixture of EPOS showers at $\log (\mathrm{E} / \mathrm{eV})=18.75\left(\left\langle\mathrm{X}_{1}\right\rangle_{C N O}=16.10 \pm 0.55 \mathrm{~g} / \mathrm{cm}^{2}\right)$

\begin{tabular}{|c|c|c|c|c|}
\hline $\mathrm{f}$ & $\begin{array}{c}\text { Sim. Value } \\
{\left[\mathrm{g} / \mathrm{cm}^{2}\right]}\end{array}$ & $\begin{array}{c}\text { ANN Value } \\
{\left[\mathrm{g} / \mathrm{cm}^{2}\right]}\end{array}$ & $\begin{array}{c}\text { Sim. Difference } \\
{\left[\mathrm{g} / \mathrm{cm}^{2}\right]}\end{array}$ & $\begin{array}{c}\text { ANN Difference } \\
{\left[\mathrm{g} / \mathrm{cm}^{2}\right]}\end{array}$ \\
\hline \hline 0.10 & $19.99 \pm 5.88$ & $13.59 \pm 1.51$ & -3.89 & 2.51 \\
\hline 0.30 & $18.33 \pm 6.60$ & $14.72 \pm 1.37$ & -2.23 & 1.38 \\
\hline 0.50 & $19.47 \pm 6.62$ & $15.95 \pm 1.79$ & -3.37 & 0.15 \\
\hline 0.70 & $24.09 \pm 4.76$ & $15.55 \pm 2.55$ & -7.99 & 0.55 \\
\hline 0.90 & $21.18 \pm 3.75$ & $16.33 \pm 4.77$ & -5.08 & -0.23 \\
\hline
\end{tabular}

Table A.50 : Calculated simulation and ANN values using pmANN+BPM for CNO in p-CNO mixture of EPOS showers at $\log (\mathrm{E} / \mathrm{eV})=18.75\left(\left\langle\mathrm{X}_{1}\right\rangle_{C N O}=16.10 \pm 0.55 \mathrm{~g} / \mathrm{cm}^{2}\right)$

\begin{tabular}{|c|c|c|c|c|}
\hline $\mathrm{f}$ & $\begin{array}{c}\text { Sim. Value } \\
{\left[\mathrm{g} / \mathrm{cm}^{2}\right]}\end{array}$ & $\begin{array}{c}\text { ANN Value } \\
{\left[\mathrm{g} / \mathrm{cm}^{2}\right]}\end{array}$ & $\begin{array}{c}\text { Sim. Difference } \\
{\left[\mathrm{g} / \mathrm{cm}^{2}\right]}\end{array}$ & $\begin{array}{c}\text { ANN Difference } \\
{\left[\mathrm{g} / \mathrm{cm}^{2}\right]}\end{array}$ \\
\hline \hline 0.10 & $19.99 \pm 5.88$ & $13.59 \pm 1.51$ & -3.89 & 2.51 \\
\hline 0.30 & $18.33 \pm 6.60$ & $14.72 \pm 1.37$ & -2.23 & 1.38 \\
\hline 0.50 & $19.47 \pm 6.62$ & $15.95 \pm 1.79$ & -3.37 & 0.15 \\
\hline 0.70 & $24.09 \pm 4.76$ & $15.55 \pm 2.55$ & -7.99 & 0.55 \\
\hline 0.90 & $21.18 \pm 3.75$ & $16.33 \pm 4.77$ & -5.08 & -0.23 \\
\hline
\end{tabular}

Table A.51 : Calculated simulation and ANN values using pmANN+BPM for CNO in p-CNO mixture of EPOS showers at $\log (\mathrm{E} / \mathrm{eV})=19.00\left(\left\langle\mathrm{X}_{1}\right\rangle_{C N O}=16.37 \pm 0.51 \mathrm{~g} / \mathrm{cm}^{2}\right)$

\begin{tabular}{|c|c|c|c|c|}
\hline $\mathrm{f}$ & $\begin{array}{c}\text { Sim. Value } \\
{\left[\mathrm{g} / \mathrm{cm}^{2}\right]}\end{array}$ & $\begin{array}{c}\text { ANN Value } \\
{\left[\mathrm{g} / \mathrm{cm}^{2}\right]}\end{array}$ & $\begin{array}{c}\text { Sim. Difference } \\
{\left[\mathrm{g} / \mathrm{cm}^{2}\right]}\end{array}$ & $\begin{array}{c}\text { ANN Difference } \\
{\left[\mathrm{g} / \mathrm{cm}^{2}\right]}\end{array}$ \\
\hline \hline 0.10 & $19.94 \pm 6.99$ & $14.19 \pm 1.65$ & -3.57 & 2.18 \\
\hline 0.30 & $17.70 \pm 6.46$ & $15.18 \pm 1.51$ & -1.33 & 1.19 \\
\hline 0.50 & $18.04 \pm 5.82$ & $15.77 \pm 1.91$ & -1.67 & 0.60 \\
\hline 0.70 & $24.17 \pm 5.01$ & $15.50 \pm 2.72$ & -7.80 & 0.87 \\
\hline 0.90 & $22.81 \pm 4.33$ & $19.26 \pm 6.41$ & -6.44 & -2.89 \\
\hline
\end{tabular}


Table A.52 : Calculated simulation and ANN values using pmANN+BPM for CNO in p-CNO mixture of EPOS showers at $\log (\mathrm{E} / \mathrm{eV})=19.00\left(\left\langle\mathrm{X}_{1}\right\rangle_{C N O}=16.37 \pm 0.51 \mathrm{~g} / \mathrm{cm}^{2}\right)$

\begin{tabular}{|c|c|c|c|c|}
\hline $\mathrm{f}$ & $\begin{array}{c}\text { Sim. Value } \\
{\left[\mathrm{g} / \mathrm{cm}^{2}\right]}\end{array}$ & $\begin{array}{c}\text { ANN Value } \\
{\left[\mathrm{g} / \mathrm{cm}^{2}\right]}\end{array}$ & $\begin{array}{c}\text { Sim. Difference } \\
{\left[\mathrm{g} / \mathrm{cm}^{2}\right]}\end{array}$ & $\begin{array}{c}\text { ANN Difference } \\
{\left[\mathrm{g} / \mathrm{cm}^{2}\right]}\end{array}$ \\
\hline \hline 0.10 & $19.94 \pm 6.99$ & $14.19 \pm 1.65$ & -3.57 & 2.18 \\
\hline 0.30 & $17.70 \pm 6.46$ & $15.18 \pm 1.51$ & -1.33 & 1.19 \\
\hline 0.50 & $18.04 \pm 5.82$ & $15.77 \pm 1.91$ & -1.67 & 0.60 \\
\hline 0.70 & $24.17 \pm 5.01$ & $15.50 \pm 2.72$ & -7.80 & 0.87 \\
\hline 0.90 & $22.81 \pm 4.33$ & $19.26 \pm 6.41$ & -6.44 & -2.89 \\
\hline
\end{tabular}

Table A.53 : Calculated simulation and ANN values using pmANN+BPM for $\mathrm{p}$ in $\mathrm{p}-\mathrm{CNO}$ mixture of EPOS showers at $\log (\mathrm{E} / \mathrm{eV})=19.50\left(\left\langle\mathrm{X}_{1}\right\rangle_{p}=38.42 \pm 1.35 \mathrm{~g} / \mathrm{cm}^{2}\right)$

\begin{tabular}{|c|c|c|c|c|}
\hline $\mathrm{f}$ & $\begin{array}{c}\text { Sim. Value } \\
{\left[\mathrm{g} / \mathrm{cm}^{2}\right]}\end{array}$ & $\begin{array}{c}\text { ANN Value } \\
{\left[\mathrm{g} / \mathrm{cm}^{2}\right]}\end{array}$ & $\begin{array}{c}\text { Sim. Difference } \\
{\left[\mathrm{g} / \mathrm{cm}^{2}\right]}\end{array}$ & $\begin{array}{c}\text { ANN Difference } \\
{\left[\mathrm{g} / \mathrm{cm}^{2}\right]}\end{array}$ \\
\hline \hline 0.10 & $19.52 \pm 1.84$ & $22.14 \pm 2.47$ & 18.90 & 16.28 \\
\hline 0.30 & $27.55 \pm 5.66$ & $31.86 \pm 7.04$ & 10.87 & 6.56 \\
\hline 0.50 & $33.47 \pm 6.03$ & $40.74 \pm 7.33$ & 4.95 & -2.32 \\
\hline 0.70 & $34.34 \pm 5.41$ & $39.24 \pm 6.04$ & 4.08 & -0.82 \\
\hline 0.90 & $34.87 \pm 4.93$ & $37.97 \pm 5.64$ & 3.55 & 0.45 \\
\hline
\end{tabular}

Table A.54 : Calculated simulation and ANN values using pmANN+BPM for CNO in p-CNO mixture of EPOS showers at $\log (\mathrm{E} / \mathrm{eV})=19.50\left(\left\langle\mathrm{X}_{1}\right\rangle_{C N O}=15.96 \pm 0.47 \mathrm{~g} / \mathrm{cm}^{2}\right)$

\begin{tabular}{|c|c|c|c|c|}
\hline $\mathrm{f}$ & $\begin{array}{c}\text { Sim. Value } \\
{\left[\mathrm{g} / \mathrm{cm}^{2}\right]}\end{array}$ & $\begin{array}{c}\text { ANN Value } \\
{\left[\mathrm{g} / \mathrm{cm}^{2}\right]}\end{array}$ & $\begin{array}{c}\text { Sim. Difference } \\
{\left[\mathrm{g} / \mathrm{cm}^{2}\right]}\end{array}$ & $\begin{array}{c}\text { ANN Difference } \\
{\left[\mathrm{g} / \mathrm{cm}^{2}\right]}\end{array}$ \\
\hline \hline 0.10 & $18.39 \pm 7.56$ & $13.68 \pm 1.27$ & -2.43 & 2.28 \\
\hline 0.30 & $15.85 \pm 6.36$ & $14.48 \pm 1.48$ & 0.11 & 1.48 \\
\hline 0.50 & $17.90 \pm 4.66$ & $16.05 \pm 2.14$ & -1.94 & -0.09 \\
\hline 0.70 & $22.21 \pm 5.61$ & $16.47 \pm 3.25$ & -6.25 & -0.51 \\
\hline 0.90 & $21.93 \pm 5.84$ & $20.42 \pm 7.13$ & -5.97 & -4.46 \\
\hline
\end{tabular}

Table A.55 : Calculated simulation and ANN values using pmANN+BPM for $\mathrm{p}$ in $\mathrm{p}-\mathrm{CNO}$ mixture of EPOS showers at $\log (\mathrm{E} / \mathrm{eV})=20.00\left(\left\langle\mathrm{X}_{1}\right\rangle_{p}=37.72 \pm 1.38 \mathrm{~g} / \mathrm{cm}^{2}\right)$

\begin{tabular}{|c|c|c|c|c|}
\hline $\mathrm{f}$ & $\begin{array}{c}\text { Sim. Value } \\
{\left[\mathrm{g} / \mathrm{cm}^{2}\right]}\end{array}$ & $\begin{array}{c}\text { ANN Value } \\
{\left[\mathrm{g} / \mathrm{cm}^{2}\right]}\end{array}$ & $\begin{array}{c}\text { Sim. Difference } \\
{\left[\mathrm{g} / \mathrm{cm}^{2}\right]}\end{array}$ & $\begin{array}{c}\text { ANN Difference } \\
{\left[\mathrm{g} / \mathrm{cm}^{2}\right]}\end{array}$ \\
\hline \hline 0.10 & $18.58 \pm 1.94$ & $20.28 \pm 2.62$ & 19.14 & 17.44 \\
\hline 0.30 & $26.98 \pm 7.28$ & $30.08 \pm 8.47$ & 10.74 & 7.64 \\
\hline 0.50 & $34.08 \pm 7.55$ & $43.50 \pm 8.66$ & 3.64 & -5.78 \\
\hline 0.70 & $33.99 \pm 6.33$ & $43.72 \pm 8.11$ & 3.73 & -6.00 \\
\hline 0.90 & $33.93 \pm 5.64$ & $43.69 \pm 7.46$ & 3.79 & -5.97 \\
\hline
\end{tabular}


Table A.56 : Calculated simulation and ANN values using pmANN+BPM for CNO in p-CNO mixture of EPOS showers at $\log (\mathrm{E} / \mathrm{eV})=20.00\left(\left\langle\mathrm{X}_{1}\right\rangle_{C N O}=15.43 \pm 0.49 \mathrm{~g} / \mathrm{cm}^{2}\right)$

\begin{tabular}{|c|c|c|c|c|}
\hline $\mathrm{f}$ & $\begin{array}{c}\text { Sim. Value } \\
{\left[\mathrm{g} / \mathrm{cm}^{2}\right]}\end{array}$ & $\begin{array}{c}\text { ANN Value } \\
{\left[\mathrm{g} / \mathrm{cm}^{2}\right]}\end{array}$ & $\begin{array}{c}\text { Sim. Difference } \\
{\left[\mathrm{g} / \mathrm{cm}^{2}\right]}\end{array}$ & $\begin{array}{c}\text { ANN Difference } \\
{\left[\mathrm{g} / \mathrm{cm}^{2}\right]}\end{array}$ \\
\hline \hline 0.10 & $19.68 \pm 7.32$ & $13.87 \pm 1.31$ & -4.25 & 1.56 \\
\hline 0.30 & $16.72 \pm 6.40$ & $14.68 \pm 1.44$ & -1.29 & 0.75 \\
\hline 0.50 & $18.10 \pm 4.18$ & $17.09 \pm 1.75$ & -2.67 & -1.66 \\
\hline 0.70 & $21.26 \pm 5.43$ & $18.32 \pm 2.49$ & -5.83 & -2.89 \\
\hline 0.90 & $21.25 \pm 6.32$ & $21.86 \pm 4.33$ & -5.82 & -6.43 \\
\hline
\end{tabular}

Table A.57 : Calculated simulation and ANN values using pmANN+BPM for $\mathrm{p}$ in $\mathrm{p}$-iron mixture of EPOS showers at $\log (\mathrm{E} / \mathrm{eV})=18.75\left(\left\langle\mathrm{X}_{1}\right\rangle_{p}=40.73 \pm 1.31 \mathrm{~g} / \mathrm{cm}^{2}\right)$

\begin{tabular}{|c|c|c|c|c|}
\hline $\mathrm{f}$ & $\begin{array}{c}\text { Sim. Value } \\
{\left[\mathrm{g} / \mathrm{cm}^{2}\right]}\end{array}$ & $\begin{array}{c}\text { ANN Value } \\
{\left[\mathrm{g} / \mathrm{cm}^{2}\right]}\end{array}$ & $\begin{array}{c}\text { Sim. Difference } \\
{\left[\mathrm{g} / \mathrm{cm}^{2}\right]}\end{array}$ & $\begin{array}{c}\text { ANN Difference } \\
{\left[\mathrm{g} / \mathrm{cm}^{2}\right]}\end{array}$ \\
\hline \hline 0.10 & $19.96 \pm 2.77$ & $20.23 \pm 3.33$ & 20.77 & 20.50 \\
\hline 0.30 & $31.54 \pm 2.21$ & $36.28 \pm 3.21$ & 9.19 & 4.45 \\
\hline 0.50 & $36.78 \pm 4.72$ & $45.54 \pm 7.19$ & 3.95 & -4.81 \\
\hline 0.70 & $37.76 \pm 4.16$ & $43.81 \pm 7.22$ & 2.97 & -3.08 \\
\hline 0.90 & $38.57 \pm 3.61$ & $41.89 \pm 6.93$ & 2.16 & -1.16 \\
\hline
\end{tabular}

Table A.58 : Calculated simulation and ANN values using pmANN+BPM for iron in p-CNO mixture of EPOS showers at $\log (\mathrm{E} / \mathrm{eV})=18.75\left(\left\langle\mathrm{X}_{1}\right\rangle_{\mathrm{Fe}}=11.09 \pm 0.75 \mathrm{~g} / \mathrm{cm}^{2}\right)$

\begin{tabular}{|c|c|c|c|c|}
\hline $\mathrm{f}$ & $\begin{array}{c}\text { Sim. Value } \\
{\left[\mathrm{g} / \mathrm{cm}^{2}\right]}\end{array}$ & $\begin{array}{c}\text { ANN Value } \\
{\left[\mathrm{g} / \mathrm{cm}^{2}\right]}\end{array}$ & $\begin{array}{c}\text { Sim. Difference } \\
{\left[\mathrm{g} / \mathrm{cm}^{2}\right]}\end{array}$ & $\begin{array}{c}\text { ANN Difference } \\
{\left[\mathrm{g} / \mathrm{cm}^{2}\right]}\end{array}$ \\
\hline \hline 0.10 & $8.29 \pm 1.59$ & $5.69 \pm 0.97$ & 2.80 & 5.40 \\
\hline 0.30 & $10.65 \pm 4.60$ & $6.75 \pm 0.94$ & 0.44 & 4.34 \\
\hline 0.50 & $17.56 \pm 7.43$ & $7.91 \pm 1.48$ & -6.47 & 3.18 \\
\hline 0.70 & $24.85 \pm 3.93$ & $8.23 \pm 1.99$ & -13.76 & 2.86 \\
\hline 0.90 & $21.26 \pm 3.86$ & $9.65 \pm 3.57$ & -10.17 & 1.44 \\
\hline
\end{tabular}

Table A.59 : Calculated simulation and ANN values using pmANN+BPM for $\mathrm{p}$ in $\mathrm{p}$-iron mixture of EPOS showers at $\log (\mathrm{E} / \mathrm{eV})=19.00\left(\left\langle\mathrm{X}_{1}\right\rangle_{p}=40.49 \pm 1.34 \mathrm{~g} / \mathrm{cm}^{2}\right)$

\begin{tabular}{|c|c|c|c|c|}
\hline $\mathrm{f}$ & $\begin{array}{c}\text { Sim. Value } \\
{\left[\mathrm{g} / \mathrm{cm}^{2}\right]}\end{array}$ & $\begin{array}{c}\text { ANN Value } \\
{\left[\mathrm{g} / \mathrm{cm}^{2}\right]}\end{array}$ & $\begin{array}{c}\text { Sim. Difference } \\
{\left[\mathrm{g} / \mathrm{cm}^{2}\right]}\end{array}$ & $\begin{array}{c}\text { ANN Difference } \\
{\left[\mathrm{g} / \mathrm{cm}^{2}\right]}\end{array}$ \\
\hline \hline 0.10 & $20.72 \pm 4.11$ & $19.70 \pm 3.96$ & 19.77 & 20.79 \\
\hline 0.30 & $32.29 \pm 3.03$ & $36.28 \pm 4.39$ & 8.20 & 4.21 \\
\hline 0.50 & $34.59 \pm 4.89$ & $43.48 \pm 6.99$ & 5.90 & -2.99 \\
\hline 0.70 & $34.61 \pm 4.23$ & $40.59 \pm 5.67$ & 5.88 & -0.10 \\
\hline 0.90 & $35.15 \pm 3.96$ & $38.85 \pm 5.09$ & 5.34 & 1.64 \\
\hline
\end{tabular}


Table A.60 : Calculated simulation and ANN values using pmANN+BPM for iron in p-CNO mixture of EPOS showers at $\log (\mathrm{E} / \mathrm{eV})=19.00\left(\left\langle\mathrm{X}_{1}\right\rangle_{F e}=9.21 \pm 0.61 \mathrm{~g} / \mathrm{cm}^{2}\right)$

\begin{tabular}{|c|c|c|c|c|}
\hline $\mathrm{f}$ & $\begin{array}{c}\text { Sim. Value } \\
{\left[\mathrm{g} / \mathrm{cm}^{2}\right]}\end{array}$ & $\begin{array}{c}\text { ANN Value } \\
{\left[\mathrm{g} / \mathrm{cm}^{2}\right]}\end{array}$ & $\begin{array}{c}\text { Sim. Difference } \\
{\left[\mathrm{g} / \mathrm{cm}^{2}\right]}\end{array}$ & $\begin{array}{c}\text { ANN Difference } \\
{\left[\mathrm{g} / \mathrm{cm}^{2}\right]}\end{array}$ \\
\hline \hline 0.10 & $7.03 \pm 1.31$ & $6.20 \pm 0.95$ & 2.18 & 3.01 \\
\hline 0.30 & $8.51 \pm 2.87$ & $7.17 \pm 0.96$ & 0.70 & 2.04 \\
\hline 0.50 & $15.14 \pm 7.51$ & $8.07 \pm 1.45$ & -5.93 & 1.14 \\
\hline 0.70 & $25.35 \pm 3.68$ & $8.13 \pm 2.00$ & -16.14 & 1.08 \\
\hline 0.90 & $22.31 \pm 3.97$ & $11.61 \pm 5.17$ & -13.10 & -2.40 \\
\hline
\end{tabular}

Table A.61 : Calculated simulation and ANN values using pmANN+BPM for $\mathrm{p}$ in p-iron mixture of EPOS showers at $\log (\mathrm{E} / \mathrm{eV})=19.50\left(\left\langle\mathrm{X}_{1}\right\rangle_{p}=38.42 \pm 1.35 \mathrm{~g} / \mathrm{cm}^{2}\right)$

\begin{tabular}{|c|c|c|c|c|}
\hline $\mathrm{f}$ & $\begin{array}{c}\text { Sim. Value } \\
{\left[\mathrm{g} / \mathrm{cm}^{2}\right]}\end{array}$ & $\begin{array}{c}\text { ANN Value } \\
{\left[\mathrm{g} / \mathrm{cm}^{2}\right]}\end{array}$ & $\begin{array}{c}\text { Sim. Difference } \\
{\left[\mathrm{g} / \mathrm{cm}^{2}\right]}\end{array}$ & $\begin{array}{c}\text { ANN Difference } \\
{\left[\mathrm{g} / \mathrm{cm}^{2}\right]}\end{array}$ \\
\hline \hline 0.10 & $19.65 \pm 4.36$ & $21.50 \pm 4.74$ & 18.77 & 16.92 \\
\hline 0.30 & $29.88 \pm 3.59$ & $33.85 \pm 4.23$ & 8.54 & 4.57 \\
\hline 0.50 & $38.96 \pm 7.52$ & $44.44 \pm 8.10$ & -0.54 & -6.02 \\
\hline 0.70 & $36.66 \pm 5.87$ & $40.52 \pm 6.41$ & 1.76 & -2.10 \\
\hline 0.90 & $35.92 \pm 4.80$ & $38.03 \pm 5.34$ & 2.50 & 0.39 \\
\hline
\end{tabular}

Table A.62 : Calculated simulation and ANN values using pmANN+BPM for iron in p-CNO mixture of EPOS showers at $\log (\mathrm{E} / \mathrm{eV})=19.50\left(\left\langle\mathrm{X}_{1}\right\rangle_{F e}=10.96 \pm 0.90 \mathrm{~g} / \mathrm{cm}^{2}\right)$

\begin{tabular}{|c|c|c|c|c|}
\hline $\mathrm{f}$ & $\begin{array}{c}\text { Sim. Value } \\
{\left[\mathrm{g} / \mathrm{cm}^{2}\right]}\end{array}$ & $\begin{array}{c}\text { ANN Value } \\
{\left[\mathrm{g} / \mathrm{cm}^{2}\right]}\end{array}$ & $\begin{array}{c}\text { Sim. Difference } \\
{\left[\mathrm{g} / \mathrm{cm}^{2}\right]}\end{array}$ & $\begin{array}{c}\text { ANN Difference } \\
{\left[\mathrm{g} / \mathrm{cm}^{2}\right]}\end{array}$ \\
\hline \hline 0.10 & $9.31 \pm 3.92$ & $7.43 \pm 0.98$ & 1.65 & 3.53 \\
\hline 0.30 & $13.02 \pm 5.94$ & $8.05 \pm 1.02$ & -2.06 & 2.91 \\
\hline 0.50 & $18.71 \pm 5.64$ & $9.51 \pm 1.61$ & -7.75 & 1.45 \\
\hline 0.70 & $22.14 \pm 5.65$ & $9.67 \pm 2.35$ & -11.18 & 1.29 \\
\hline 0.90 & $21.63 \pm 5.55$ & $13.20 \pm 6.04$ & -10.67 & -2.24 \\
\hline
\end{tabular}

Table A.63 : Calculated simulation and ANN values using pmANN+BPM for $\mathrm{p}$ in $\mathrm{p}$-iron mixture of EPOS showers at $\log (\mathrm{E} / \mathrm{eV})=20.00\left(\left\langle\mathrm{X}_{1}\right\rangle_{p}=37.72 \pm 1.38 \mathrm{~g} / \mathrm{cm}^{2}\right)$

\begin{tabular}{|c|c|c|c|c|}
\hline $\mathrm{f}$ & $\begin{array}{c}\text { Sim. Value } \\
{\left[\mathrm{g} / \mathrm{cm}^{2}\right]}\end{array}$ & $\begin{array}{c}\text { ANN Value } \\
{\left[\mathrm{g} / \mathrm{cm}^{2}\right]}\end{array}$ & $\begin{array}{c}\text { Sim. Difference } \\
{\left[\mathrm{g} / \mathrm{cm}^{2}\right]}\end{array}$ & $\begin{array}{c}\text { ANN Difference } \\
{\left[\mathrm{g} / \mathrm{cm}^{2}\right]}\end{array}$ \\
\hline \hline 0.10 & $18.08 \pm 4.79$ & $20.93 \pm 5.57$ & 19.64 & 16.79 \\
\hline 0.30 & $28.72 \pm 3.90$ & $31.30 \pm 4.45$ & 9.00 & 6.42 \\
\hline 0.50 & $38.58 \pm 7.90$ & $46.89 \pm 9.03$ & -0.86 & -9.17 \\
\hline 0.70 & $36.13 \pm 6.04$ & $44.10 \pm 7.85$ & 1.59 & -6.38 \\
\hline 0.90 & $35.03 \pm 4.96$ & $42.06 \pm 7.04$ & 2.69 & -4.34 \\
\hline
\end{tabular}


Table A.64 : Calculated simulation and ANN values using pmANN+BPM for iron in p-CNO mixture of EPOS showers at $\log (\mathrm{E} / \mathrm{eV})=20.00\left(\left\langle\mathrm{X}_{1}\right\rangle_{F e}=9.98 \pm 0.74 \mathrm{~g} / \mathrm{cm}^{2}\right)$

\begin{tabular}{|c|c|c|c|c|}
\hline $\mathrm{f}$ & $\begin{array}{c}\text { Sim. Value } \\
{\left[\mathrm{g} / \mathrm{cm}^{2}\right]}\end{array}$ & $\begin{array}{c}\text { ANN Value } \\
{\left[\mathrm{g} / \mathrm{cm}^{2}\right]}\end{array}$ & $\begin{array}{c}\text { Sim. Difference } \\
{\left[\mathrm{g} / \mathrm{cm}^{2}\right]}\end{array}$ & $\begin{array}{c}\text { ANN Difference } \\
{\left[\mathrm{g} / \mathrm{cm}^{2}\right]}\end{array}$ \\
\hline \hline 0.10 & $7.97 \pm 3.34$ & $7.83 \pm 1.00$ & 2.01 & 2.15 \\
\hline 0.30 & $11.02 \pm 4.76$ & $8.67 \pm 1.05$ & -1.04 & 1.31 \\
\hline 0.50 & $16.68 \pm 5.36$ & $11.11 \pm 1.75$ & -6.70 & -1.13 \\
\hline 0.70 & $21.81 \pm 5.85$ & $12.39 \pm 2.44$ & -11.83 & -2.41 \\
\hline 0.90 & $22.23 \pm 6.03$ & $16.52 \pm 4.25$ & -12.25 & -6.54 \\
\hline
\end{tabular}

Table A.65 : Calculated simulation and ANN values using pmANN+BPM for CNO in CNO-iron mixture of EPOS showers at $\log (\mathrm{E} / \mathrm{eV})=18.75\left(\left\langle\mathrm{X}_{1}\right\rangle_{C N O}=16.10 \pm 0.55 \mathrm{~g} / \mathrm{cm}^{2}\right)$

\begin{tabular}{|c|c|c|c|c|}
\hline $\mathrm{f}$ & $\begin{array}{c}\text { Sim. Value } \\
{\left[\mathrm{g} / \mathrm{cm}^{2}\right]}\end{array}$ & $\begin{array}{c}\text { ANN Value } \\
{\left[\mathrm{g} / \mathrm{cm}^{2}\right]}\end{array}$ & $\begin{array}{c}\text { Sim. Difference } \\
{\left[\mathrm{g} / \mathrm{cm}^{2}\right]}\end{array}$ & $\begin{array}{c}\text { ANN Difference } \\
{\left[\mathrm{g} / \mathrm{cm}^{2}\right]}\end{array}$ \\
\hline \hline 0.10 & $11.85 \pm 1.47$ & $13.18 \pm 1.21$ & 4.25 & 2.92 \\
\hline 0.30 & $13.98 \pm 1.34$ & $15.12 \pm 1.28$ & 2.12 & 0.98 \\
\hline 0.50 & $15.19 \pm 1.22$ & $16.58 \pm 1.27$ & 0.91 & -0.48 \\
\hline 0.70 & $16.01 \pm 1.17$ & $17.54 \pm 1.26$ & 0.09 & -1.44 \\
\hline 0.90 & $16.55 \pm 1.12$ & $18.24 \pm 1.23$ & -0.45 & -2.14 \\
\hline
\end{tabular}

Table A.66 : Calculated simulation and ANN values using pmANN+BPM for iron in CNO-iron mixture of EPOS showers at $\log (\mathrm{E} / \mathrm{eV})=18.75\left(\left\langle\mathrm{X}_{1}\right\rangle_{F e}=11.09 \pm 0.75 \mathrm{~g} / \mathrm{cm}^{2}\right)$

\begin{tabular}{|c|c|c|c|c|}
\hline $\mathrm{f}$ & $\begin{array}{c}\text { Sim. Value } \\
{\left[\mathrm{g} / \mathrm{cm}^{2}\right]}\end{array}$ & $\begin{array}{c}\text { ANN Value } \\
{\left[\mathrm{g} / \mathrm{cm}^{2}\right]}\end{array}$ & $\begin{array}{c}\text { Sim. Difference } \\
{\left[\mathrm{g} / \mathrm{cm}^{2}\right]}\end{array}$ & $\begin{array}{c}\text { ANN Difference } \\
{\left[\mathrm{g} / \mathrm{cm}^{2}\right]}\end{array}$ \\
\hline \hline 0.10 & $10.70 \pm 4.78$ & $7.59 \pm 4.33$ & 0.39 & 3.50 \\
\hline 0.30 & $13.79 \pm 6.38$ & $6.52 \pm 3.60$ & -2.70 & 4.57 \\
\hline 0.50 & $16.22 \pm 6.41$ & $7.32 \pm 3.76$ & -5.13 & 3.77 \\
\hline 0.70 & $19.22 \pm 6.17$ & $8.29 \pm 2.90$ & -8.13 & 2.80 \\
\hline 0.90 & $19.53 \pm 4.71$ & $11.02 \pm 4.84$ & -8.44 & 0.07 \\
\hline
\end{tabular}


Table A.67 : Calculated simulation and ANN values using pmANN+BPM for CNO in CNO-iron mixture of EPOS showers at $\log (\mathrm{E} / \mathrm{eV})=19.00\left(\left\langle\mathrm{X}_{1}\right\rangle_{C N O}=16.37 \pm 0.51 \mathrm{~g} / \mathrm{cm}^{2}\right)$

\begin{tabular}{|c|c|c|c|c|}
\hline $\mathrm{f}$ & $\begin{array}{c}\text { Sim. Value } \\
{\left[\mathrm{g} / \mathrm{cm}^{2}\right]}\end{array}$ & $\begin{array}{c}\text { ANN Value } \\
{\left[\mathrm{g} / \mathrm{cm}^{2}\right]}\end{array}$ & $\begin{array}{c}\text { Sim. Difference } \\
{\left[\mathrm{g} / \mathrm{cm}^{2}\right]}\end{array}$ & $\begin{array}{c}\text { ANN Difference } \\
{\left[\mathrm{g} / \mathrm{cm}^{2}\right]}\end{array}$ \\
\hline \hline 0.10 & $11.56 \pm 1.81$ & $12.61 \pm 1.43$ & 4.81 & 3.76 \\
\hline 0.30 & $13.39 \pm 1.72$ & $14.50 \pm 1.47$ & 2.98 & 1.87 \\
\hline 0.50 & $14.70 \pm 1.60$ & $15.81 \pm 1.44$ & 1.67 & 0.56 \\
\hline 0.70 & $15.56 \pm 1.41$ & $16.73 \pm 1.43$ & 0.81 & -0.36 \\
\hline 0.90 & $16.06 \pm 1.28$ & $17.46 \pm 1.36$ & 0.31 & -1.09 \\
\hline
\end{tabular}

Table A.68 : Calculated simulation and ANN values using pmANN+BPM for iron in CNO-iron mixture of EPOS showers at $\log (\mathrm{E} / \mathrm{eV})=19.00\left(\left\langle\mathrm{X}_{1}\right\rangle_{F e}=9.21 \pm 0.61 \mathrm{~g} / \mathrm{cm}^{2}\right)$

\begin{tabular}{|c|c|c|c|c|}
\hline $\mathrm{f}$ & $\begin{array}{c}\text { Sim. Value } \\
{\left[\mathrm{g} / \mathrm{cm}^{2}\right]}\end{array}$ & $\begin{array}{c}\text { ANN Value } \\
{\left[\mathrm{g} / \mathrm{cm}^{2}\right]}\end{array}$ & $\begin{array}{c}\text { Sim. Difference } \\
{\left[\mathrm{g} / \mathrm{cm}^{2}\right]}\end{array}$ & $\begin{array}{c}\text { ANN Difference } \\
{\left[\mathrm{g} / \mathrm{cm}^{2}\right]}\end{array}$ \\
\hline \hline 0.10 & $8.58 \pm 4.86$ & $6.74 \pm 3.20$ & 0.63 & 2.47 \\
\hline 0.30 & $11.54 \pm 6.64$ & $7.48 \pm 3.37$ & -2.33 & 1.73 \\
\hline 0.50 & $14.08 \pm 6.95$ & $8.32 \pm 3.29$ & -4.87 & 0.89 \\
\hline 0.70 & $17.15 \pm 6.89$ & $9.91 \pm 3.71$ & -7.94 & -0.70 \\
\hline 0.90 & $18.50 \pm 6.02$ & $11.84 \pm 4.28$ & -9.29 & -2.63 \\
\hline
\end{tabular}

Table A.69 : Calculated simulation and ANN values using pmANN+BPM for CNO in CNO-iron mixture of EPOS showers at $\log (\mathrm{E} / \mathrm{eV})=19.50\left(\left\langle\mathrm{X}_{1}\right\rangle_{C N O}=15.96 \pm 0.47 \mathrm{~g} / \mathrm{cm}^{2}\right)$

\begin{tabular}{|c|c|c|c|c|}
\hline $\mathrm{f}$ & $\begin{array}{c}\text { Sim. Value } \\
{\left[\mathrm{g} / \mathrm{cm}^{2}\right]}\end{array}$ & $\begin{array}{c}\text { ANN Value } \\
{\left[\mathrm{g} / \mathrm{cm}^{2}\right]}\end{array}$ & $\begin{array}{c}\text { Sim. Difference } \\
{\left[\mathrm{g} / \mathrm{cm}^{2}\right]}\end{array}$ & $\begin{array}{c}\text { ANN Difference } \\
{\left[\mathrm{g} / \mathrm{cm}^{2}\right]}\end{array}$ \\
\hline \hline 0.10 & $11.51 \pm 2.37$ & $13.41 \pm 2.16$ & 4.45 & 2.55 \\
\hline 0.30 & $13.21 \pm 2.14$ & $15.69 \pm 2.27$ & 2.75 & 0.27 \\
\hline 0.50 & $14.24 \pm 1.95$ & $17.32 \pm 2.13$ & 1.72 & -1.36 \\
\hline 0.70 & $15.02 \pm 1.83$ & $18.42 \pm 2.02$ & 0.94 & -2.46 \\
\hline 0.90 & $15.70 \pm 1.77$ & $19.23 \pm 1.84$ & 0.26 & -3.27 \\
\hline
\end{tabular}


Table A.70 : Calculated simulation and ANN values using pmANN+BPM for iron in CNO-iron mixture of EPOS showers at $\log (\mathrm{E} / \mathrm{eV})=19.50\left(\left\langle\mathrm{X}_{1}\right\rangle_{F e}=10.96 \pm 0.90 \mathrm{~g} / \mathrm{cm}^{2}\right)$

\begin{tabular}{|c|c|c|c|c|}
\hline $\mathrm{f}$ & $\begin{array}{c}\text { Sim. Value } \\
{\left[\mathrm{g} / \mathrm{cm}^{2}\right]}\end{array}$ & $\begin{array}{c}\text { ANN Value } \\
{\left[\mathrm{g} / \mathrm{cm}^{2}\right]}\end{array}$ & $\begin{array}{c}\text { Sim. Difference } \\
{\left[\mathrm{g} / \mathrm{cm}^{2}\right]}\end{array}$ & $\begin{array}{c}\text { ANN Difference } \\
{\left[\mathrm{g} / \mathrm{cm}^{2}\right]}\end{array}$ \\
\hline \hline 0.10 & $9.49 \pm 5.76$ & $7.59 \pm 1.97$ & 1.47 & 3.37 \\
\hline 0.30 & $12.00 \pm 6.31$ & $8.00 \pm 1.37$ & -1.04 & 2.96 \\
\hline 0.50 & $13.64 \pm 6.52$ & $8.59 \pm 1.27$ & -2.68 & 2.37 \\
\hline 0.70 & $15.36 \pm 6.71$ & $9.36 \pm 1.31$ & -4.40 & 1.60 \\
\hline 0.90 & $16.35 \pm 6.68$ & $10.43 \pm 1.73$ & -5.39 & 0.53 \\
\hline
\end{tabular}

Table A.71 : Calculated simulation and ANN values using pmANN+BPM for CNO in CNO-iron mixture of EPOS showers at $\log (\mathrm{E} / \mathrm{eV})=20.00\left(\left\langle\mathrm{X}_{1}\right\rangle_{C N O}=15.43 \pm 0.49 \mathrm{~g} / \mathrm{cm}^{2}\right)$

\begin{tabular}{|c|c|c|c|c|}
\hline $\mathrm{f}$ & $\begin{array}{c}\text { Sim. Value } \\
{\left[\mathrm{g} / \mathrm{cm}^{2}\right]}\end{array}$ & $\begin{array}{c}\text { ANN Value } \\
{\left[\mathrm{g} / \mathrm{cm}^{2}\right]}\end{array}$ & $\begin{array}{c}\text { Sim. Difference } \\
{\left[\mathrm{g} / \mathrm{cm}^{2}\right]}\end{array}$ & $\begin{array}{c}\text { ANN Difference } \\
{\left[\mathrm{g} / \mathrm{cm}^{2}\right]}\end{array}$ \\
\hline \hline 0.10 & $11.62 \pm 2.52$ & $14.15 \pm 2.43$ & 3.81 & 1.28 \\
\hline 0.30 & $12.58 \pm 2.30$ & $16.01 \pm 2.40$ & 2.85 & -0.58 \\
\hline 0.50 & $13.67 \pm 2.22$ & $17.10 \pm 2.19$ & 1.76 & -1.67 \\
\hline 0.70 & $14.53 \pm 1.96$ & $17.88 \pm 2.01$ & 0.90 & -2.45 \\
\hline 0.90 & $15.11 \pm 1.81$ & $18.49 \pm 1.85$ & 0.32 & -3.06 \\
\hline
\end{tabular}

Table A.72 : Calculated simulation and ANN values using pmANN+BPM for iron in CNO-CNO mixture of EPOS showers at $\log (\mathrm{E} / \mathrm{eV})=20.00\left(\left\langle\mathrm{X}_{1}\right\rangle_{F e}=9.98 \pm 0.74 \mathrm{~g} / \mathrm{cm}^{2}\right)$

\begin{tabular}{|c|c|c|c|c|}
\hline $\mathrm{f}$ & $\begin{array}{c}\text { Sim. Value } \\
{\left[\mathrm{g} / \mathrm{cm}^{2}\right]}\end{array}$ & $\begin{array}{c}\text { ANN Value } \\
{\left[\mathrm{g} / \mathrm{cm}^{2}\right]}\end{array}$ & $\begin{array}{c}\text { Sim. Difference } \\
{\left[\mathrm{g} / \mathrm{cm}^{2}\right]}\end{array}$ & $\begin{array}{c}\text { ANN Difference } \\
{\left[\mathrm{g} / \mathrm{cm}^{2}\right]}\end{array}$ \\
\hline \hline 0.10 & $8.83 \pm 4.98$ & $7.81 \pm 2.17$ & 1.15 & 2.17 \\
\hline 0.30 & $11.14 \pm 6.21$ & $8.29 \pm 1.40$ & -1.16 & 1.69 \\
\hline 0.50 & $12.15 \pm 6.14$ & $8.84 \pm 1.69$ & -2.17 & 1.14 \\
\hline 0.70 & $15.01 \pm 6.87$ & $9.56 \pm 1.89$ & -5.03 & 0.42 \\
\hline 0.90 & $16.10 \pm 6.78$ & $10.23 \pm 2.20$ & -6.12 & -0.25 \\
\hline
\end{tabular}

